3.

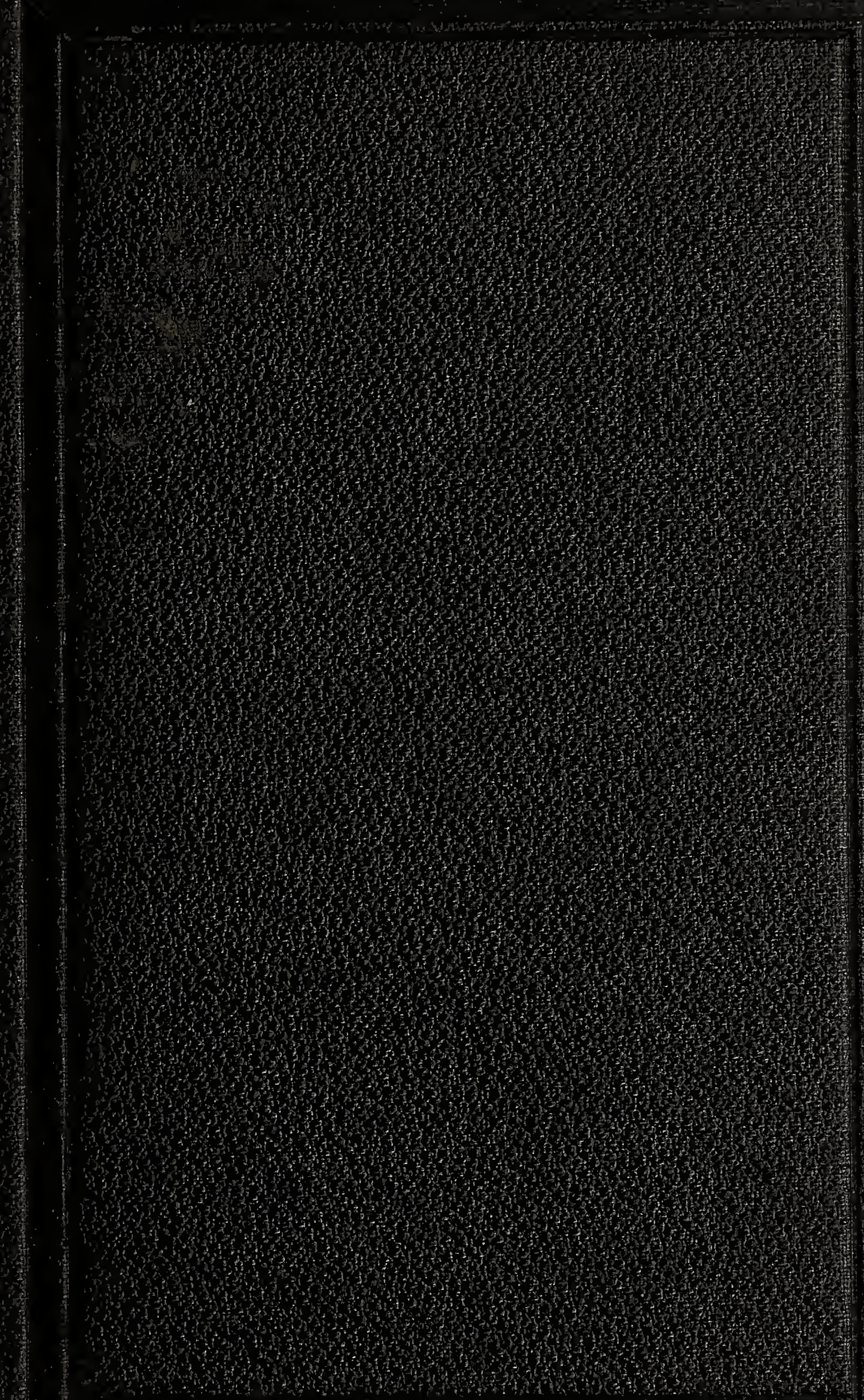

is

(x)

97

4

as

3.

1)

(1)

(1)

,

H.

,

3.

(1)

5.

开

H.

4

H.

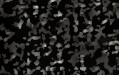

in

W

15

3

1y

40

3.

3

3

4

\%

$3 x$



4

X

3

37

4

15 

lang. 
bymm - beote in the

Eakime of fehador 
$P M$

64

I 33

1879

SOA

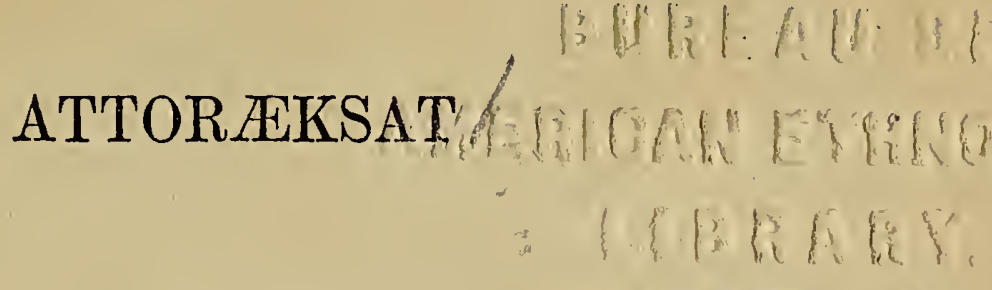 \\ IOI39
}

LABRADORE ME TUNUT./

Etymom-book

Esteino y Labrador

nom

STOLPENEME,

G. WINTERIB NÊNILAUKTANGIT.

1879. 


\section{PM64 I6}

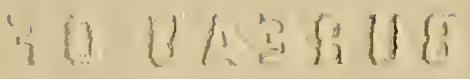

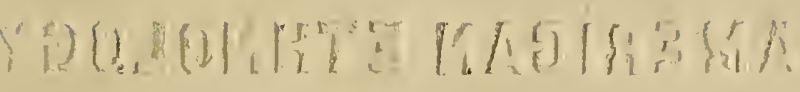

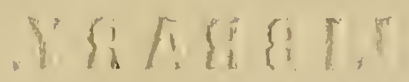




\section{Vorbemerkung.}

Diese neue Auflage ist sowol um ganze Lieder, als einzelne Verse - zusammen etwa 140 Verse - vermehrt. Um die alte Ausgabe noch weiter gebrauchen zu können, sind die alten Nummern beibehalten worden, wobei dann die neuen Lieder noch einen Buchstaben zur Nummer erhalten haben, z. B. 172 a. Die neuen einzelnen Verse sind durch ein * kenntlich gemacht. Durch solche Verse hat sich dann die Verszahl in einigen alten Liedern verändert, worauf der Liturgus achten möge.

In den Ueberschriften sind die Nummern der betreffenden übersetzten deutsehen Lieder hinzugefügt worden, und zwar nach dem sogenannten „Kleinen Gesangbuch“. Ein * bezeichnet hier das alte grössere Gesangbuch und seinen Anhang (Nachtrag). Bei den englischen Liedern weisen die Nummern auf unser englisches Brüdergesangbuch.

So lang die zwei Auflagen des Gesangbuchs nebeneinander in Gebrauch sind, wird es sich, um mögliche Störungen zu vermeiden, empfehlen, (was ja überhaupt anzuraten ist), dass der Liturgus beim Nennen der Nummer auch stets den Anfang des Verses mit nennt.

Was nun die neuen Lieder und Verse betrifft, so ist der Vers 731 a. Herr und Haupt Immanuel, uns eine Erinnerung an unsern seligen nun vor schon 11 Jahren heimgegangenen Br. Freitag. Ebenso sind die Lieder *.

531 a. Auf, ihr Christen überwindet,

744 a. Dir, Dir Jehova, will ich singen,

830 a. Es halten eitele Gemüter,

234 a. Es ist vollbracht, was willst du nun,

$833 \mathrm{~b}$. Heilger Bräutgam unsrer Seelen - 
eine Erinnerung an unsern bisherigen Mitarbeiter, Br. Elsner, der leider vor der Zeit sich genötigt sieht, in diesem Jahre seinen Dienst allhier zu beschliessen. Die andern neuen Verse und Lieder sind folgende:

47 a. Den die Engel droben,

51 a. 0 du holder, süsser Knabe,

54 a. Ein schönes Weihnachtslied aus dem Dänischen,

42 a-e. Weihnachtslieder aus dem Englischen,

112, 2. Ich umfange, herz' und küsse (aus dem Liturgieenbuch),

158 a. Wenn der Herr, der aus dem Grab erwachte,

158 b. Welche Gottesgegenwart (ähnlich wie im Liturgieenbuch),

283 a. Eines wünsch' ich mir vor allen andern,

305 a. Alle deine Gaben,

320, 3. Halleluja sei Dir gesungen,

325, 1. Sollt ich nicht von Lieb und Dank entglommen,

472 a. Heilger Geist voll Gnad und Liebe,

485 a. Heiligster Jesu, Heil'gungsquelle,

519 a. Gott wills machen,

537 a. Die Gnade sei mit allen,

646, 5. Dein Fleisch muss mns zum Pfande dienen,

742 a. From Greenlands icy mountains,

808 5, Tretet nur getrost zum Throne,

814 a. 0 ihr Gottesheere,

833 a. Es ist noch eine Ruh vorhanden,

857,2 . Drum üherwindt mein Glaube weit.

Ausserdem: $\quad 148,3-5 . \quad 149$ a. $163,2-5 . \quad 172$. a-h. 247, 1. 269, 1. 525,3. 803,1.2 (aus dem Liturgieenbuch). 814 b. 865 a.

Die Erfahrung ist wol allgemein gemacht worden, dass in manchen Fällen, z. B. bei alttestamentlichen Bibellektionen, passende 


\section{Vorbemerkung.}

Verse allgemeineren Inhalts schwer aufzutreiben waren. Für solche Fälle werden hoffentlich manchen die Lieder 172. a-h ganz willkommen sein.

Das Lied 42 a. "Attê tuppaleritse" ist ein altes englisches Lied: Christians awake u. s. w. Dies Lied pflegt in unsern englischen Gemeinen (in allen? ${ }^{*}$ ) am Weihnachtsmorgen in der ersten Versammlung stehend gesungen zu werden. Vielleicht könnten Verse davon in ähnlicher Weise bei unsrer Frühversammlung des Anbetens verwendet werden. Die Melodie darf man nur nicht zu langsam nehmen.

Ausser dieser Melodie 600 sind als in unserm Gesangbuch noch nicht vertreten, in dieser neuen Auflage aber aufgenommen Melodien zu nennen:

7. 110. 130. 166 b. 208 d. 585. b. 595 a.

Möge nun unser Herr und Heiland Ġnade geben, dass die in diesem Buche enthaltenen Worte der Bitte, des Gebetes, des Lobens und Dankens beim Gebrauch nicht nur ein Bekenntnis des Mundes bleiben, sondern ein ihm wolgefälliges, lebendiges Opfer des Herzens werden!

NAIN, den 29sten Juni 1878.

*) Wird auch sehr allgemein in Städten und Dörfern in der Nacht vor dem Weihnachtsmorgen im Freien von herumwandelnden Gesellschaften aus Männern und Knaben bestehend gesungen, mit oder ohne Instrumentalbegleitung.

H. E. Shawe. 


\section{IMGERUTIT}

\section{IMGMIGÔLINGANINGITA TUKKEKARNINGINIK.}

1. Gûdib orausinginik . . . . . . . . . 1-15

2. Inuit kattaksimaningænnik, Kristusemullo piulijauningænnik . . . • . . . . 16-22

3. Kristusib inûlerninganik uviningmelo sakKerninganik . . . . . . . . 23-30 4. Jêsusib Kristusib erniangoninganik arvertarninganiglo nuname $. \cdot . \cdot \quad \cdot . * 31-69$

5. Jêsusib Kristusib kippijauninganik Jâriblo nutâb pigiarningane imgerutinik . . . 70-81

6. Kristusib ânianganik, toKkunganiglo illuvertauninganiglo

$82-146$

7. Kristusib makkininganik illuverminit . . 147-158

8. Kristusib kilalliarninganik iksivaninganiglo

Gûdib tallerpiane . . . . . . . 159-167

9. Gûdemik, sakxijârninganiglo pingortitsinerme . . . . . . . . . . 168-176

10. Gûdemik pingasôlijomik . . . . . . . 177-182

11. Nâlegapta Jêsusib Kristusib Atâtanganik Atâtagijaptingniktauk . . . . . 183-190

12. Gûdib Erninganik, Nâlegaptingnik Piulijiptingniglo : . . . • . . . . . . 191-197

13. Anernermik ajunginermik, tâpsomalo tunnergutinganik piniarninganiglo . . . . 198-206

14. Gûdib Kaikojininganik, ajortut sâgiarkovlugit . . $. . . \quad . \quad . \quad \therefore \quad .207-214$

15. Kakкialernermik piulijaujomanek pitjutigilugo . . . . . . . . . . . 215-230 16. Okpernermik Jêsusemut . . . . . 231-253 
17. Ajornernit issumagijaujungnainermik . . . 25̌4-270.

18. Omamut illuitomut tunninermik Jêsusemut 271-281

19. Kristusemik tarnib illakaromaninganik . . 282-299

20. Ullapirsaumik, kuviasungnermiglo Anernerme ajunginerme . . . . . . . 300-318.

21. Gûdib - kittorngangita piloringninginik . 319-327

22. Jêsuseme Kristuseme inôtsemik nellâgôrtomik . . . . . . . . . . . . 328-335

23. Mallingnermik Jêsusemik, illangiutinermiglo siorniorninganik . . . . . . . . 336-345

24. Jêsusib naglingninganik inungnik, piluarlunelo inukotiminik. . . . . . . 346-363 25. Jêsusib inûlerninga tokкungalo pivlugik, ômamut kujalinermik

26. Jêsusib ikkinginik okpernermut tækkonârnermik . . . . . . . . . . . 390-414

27. Jêsusemut naglingnermik, tâpsomungalo kippiniarnermik

28. Kattangutinut naglingnermik attausemiglo issumakattigêngermik . . . . . . .438-448

29. Ingminik illitarsivallianermik annersauminer-

$$
\text { miglo saimartitaujomanermut }
$$

30. Ivsornaititsinermik timemiglo tarnemiglo

$364-389$

31. Kristusemut okpernermik issumalingasuêr-

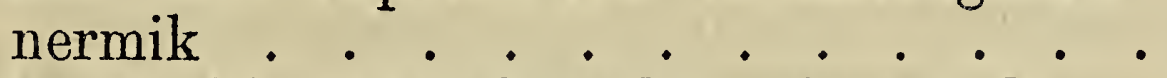

32. Omattib akluninganik, nellokotinermiglo . 505-509

33. Sokкosijuinermiglo saimartitauvallianermiglo $510-517$

34. Kiksarnartome Kennuêrsârnermik manigor-

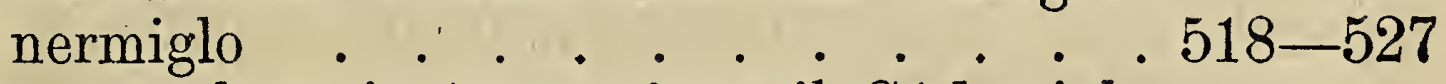

35. Okpernerub kuviasûtekarninganik Gûdemiglo tættekarnermik

36. Ônatib uimajângininganik . . . . . . . . . 538-5371

37. Okpertunik illûnânêtunik, illagêktunit anginersamik .

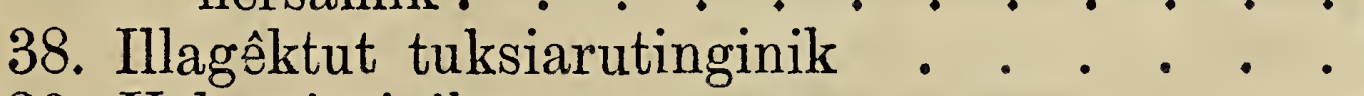

39. Uvloksiutinik

40. Baptijumik hailigemik . 
VIII Imgerutit ingmigôlinganingita etc.

41. Komunionemik hailigemilk . • . . .604-656

42. Ingmikôrtartut imgerutinginik.

(a) Ingmikôrtartunut illûnainut. • . . 657-662

(b) Sorrutsit imgerutinginik . . . . 663-676

(c) Nukapitset Nullêtullo imgerutinginik 677-684

(d) Niviarsiat uigasuillo imgerutinginik . 685-692

(e) Nulliarêt imgerutingnik . . . 693-703

(f) Uigarnerit imgerutinginik . . . . 704-711

43. Nâlekab illagêktullo kivganginilk • • . . 712-727

44. Kristusib nâlegauvingat oKautauninganik,

tillijanginnut kigligiudsiningnullo nuname $728-743$ 45. Kennunermik, tuksiarnermiglo tuksiarutjinermiglo.

(a) Nangminek pivlune, assivullo pivlugit 744-754

(b) Attanivullo pivlugit . . . . . 755-756 46. Imgerutinik uvlâkut. . . . . . . . . 757-772 47. Imgerutinilk piniarnermik . . . . . . .773-777 48. Nerrimârsiutinik imgerutinik. . . . . . 778-780 49. Nerrijarêrnermik imgerutinik . . . . .781-782 50. Ingergarnermik imgerutinik . . . . . . 783-784 51. Unnuktome imgerutinik . . . . . . .785-795 52. Engelinik . . . . . . . . . . 796-801

53. Imgerutinik nertordlerutiksanik kujalitiksaniglo . . . . . . . . . . . . . 802-816 54. Kristusib tikkitsomârninganik ânanaunermine, ômingalo nerriungniptingnik . . . . . 817-829 55. Anerniptigut arvertarniptingnik kilangme, Kristusemêtsomavlutalo killanârnermik . 830-835 56. Nâlekamut angergarnermik, timiblo makkitsomârninganik .

57. Angergarsimajunik illagêktunik, nâksaungito$\begin{gathered}\text { miglo ânanaunermik. } \\ \text { 58. Inngerutinik sorairutiksanik }\end{gathered} . \quad . \quad . \quad . \quad . \quad .856-863$ 59. Imgerutinik saimartitsinermut . . . . 867-882 


\section{MELODIT NIPPISIORUTINGIT,}

\section{SORLO ILLAGÊKTUNE KATTANGUTIGÊNIK TAI- JAUJUNE ATTORTAUVANGMATTA.}

Mel.

1. Jêsuse sulle nagligosuktotit, No. 211. 264. 778. 781.

4. Gûb Anerninga, 202. 203. 242. 265. 288. 465.673.

7. Kaitatit illûnatik, 47 a. 172 c. 305 a.

8. Nâpkiningnernut Jêsus, 172 d. 537 a. 866.

9. Jêsub tækkosarlise, 81. 117. 133. 190. 401. 577. 593. 608. 850.861 .

10. Sekkerngub miksekârtub, 52. $53 . \quad 77 . \quad 79 . \quad 88.158$. 184. 357. 391. 513. 675. 699. 724. 768. 782. 868. 873. 876.

11. Jêsus kailaurit mânna (a), Gûd nakoridlartaule (b), 25. 29. 69. 170. 171. 204. 245. 428. 438. 633. 750. 870.

14. Ômatimnik kaitsivunga, 22. 37. 49. 58. 90. 93. 107. 140. 257. 276. 279. 299. 442. 496. 503. 521. 527. 541. 599. 607. 612. 666. 705. 722. 766. 776. 807. 15. Aniarnut tokkungnullo, 154. 237. 285. 358. 375. 376. 424. 443. 449. 698. 862.

16. Gûdib ajokertûsingit, 11. 149. 194. 247. 263. 269. 338. 340. 488. 490. 491. 502. 519 a. 697. 707. 819.

20. Jêsusiga erKarupkit, 419. 664.

22. Kristum aunga idluartok (a) Gûde ajugakangitok (b), 4. 21. 23. 28. 31. 32. 33. 36. 39. 57. 74. 80. 125. 166. 188. 197. 206. 216. 236. 239. 252. 267. 326 . 355. 366. 478. 4ะ4. 487. 508. 519. 568. 602. 606. 611. 621. 625. 642. 650. 652. 668. 671. 678. 687. 689. 691. 758. 771. 783. 787. 796. 842. 865. 869. 
Mel.

23. Illûnapkut ajorpunga, 126. 390. 396. 600. 617. 620 . 632. 648. 651. 653. 659. 780. 852. 872.

26. Tarninik naglingniktotit, 343. 463. 546. 553. 571. 586. 638. 706. 716.

29. Nunamêtillunga Jêsuse, 246. 332. 511. 723. 742.

30. Aggaktit puttomajut issakit, 470. 474. 530. 578. 594.

32. Piulijiga âniadlartok, 106. 629.

36. NagliktoK Jêsuse sumik pilaurêt, 82. 89. 96. 102. 220. 231. 291. 3533. 377. 536. 614. 622. 747. 794. 37. Nagliktok Jêsuse illingnêlanga, 233. 273. 281. 303. 450. 493. 500. 550. 657. 677. 711. 720. 835.

39. Gûud, Gûdivut Kattangutigijavullo, 55. 151. 212. 287. 430. 431. 674. 729. 798. 816.

45. Nâpkiningnit angidlarpoк, 173. 363.

50. Issokangitor kaumajok, 34 .

51. Jêsus kikkiæktulaungmat, 116.

54. Illagêt illagisiuk, 46. 83. 100. 167. 526.

56. Saimartigut piulijivut. 489. 562. 570. 598 .

58. Gûb Anerninga Kennuvavut, 2. 54. 72. 127. 187. 199 201. 270. 289. 345. 365. 379. 406. 435. 544. 640. 658. 681. 718. 728. 743. 813. 826. 849. 854. 881.

61. Kilangmit nunamut akkalerkêt Jêsusiga, 43. 802.

64. Puailavit tellimauvut, 123.

68. Gûbb saugârsunga piulijiga, 8. 195. 337. 352. 399. 409. 760. 792.

69. Kuvianadiarmêk Nâlekab iglungit, 543. 624. 639 .

70. Aulik Nâlegar, 24. 78. 278. 579. 631. 636. 661. 669. 713. 730. 733. 797.

71. Opallungailerit, tarne, Piulijît, 628. 821.

75. Namut sâniarpîk, 224. 227. 250. 312. 378. 397. 404. 790. 822. 832.

79. Illagêktut okorse, 104. 110. 137. 175. 205. 248. 277. 354. 360. 382. 393. 407. 436. 447. 457. 459. 472. 475. 481. 539. 563. 573. 635. 662. 663. 670. 688. 696. 704. 710. 731. 740. 748. 749. 765. 773. 793. 795. 825. 843. 867. 871. 875 . 
Mel.

82. Gûb kittorngaringmanga, 183. 218. 272. 314. 368. 410. 647.

83. Jêsus tættigijara, 59. 221. 259. 420. 425. 518. 558. 613. 703. 831. 838.

84. Jêsuse mânêpogut, 3. 603. 777.

89. Anerner ômartitsijok, 200. 761.

90. Kristusemiut tamapse, 172 e. 336. 413. 812.

95. Nagliktiga Jêsusiga, 51. 51 a. 307. 732.

96. Atâtak kilangmêtotit, 186 .

97. Niakок Gûd pairijivut, 207. 261. 322. 350. 385. 418. 448. 461. 469. 512. 609. 630. 755. 784. 857.

99. Jêsusiga, tautukapkit, 84. 145. 610.

101. Nellopкotimut kaititse, 359.

102. Pairsijotôgavit illa, 557. 649.

106. Gûdemik tættetuakartok, 174. 210. 244. 275. 422. 485. 534. 830. 844. 880.

107. Jêsusigale tokкokârnak, 646.

109. Aulajeкattautigilakka, 129.

110. Jehôva imgervigivagit 744 a.

112. Tussarpâtit, 525.

114. Uvamnigle pairksigavit Gûdiga, 147. 306. 364. 548. 589. 701.752.

115. KittorngauneK Kristusemut ânanâk, 14. 301. 321. 330. 517.

119. Hailig Gûd, 1. 372. 751. 779. 859.

121. Imanuêliga nagliguk tarniga, 249. 369. 384. 446. 468. 506. 567. 591. 654. 739. 775.

123. Kaumaksalaungminga, 228.

124. KanoktôK illuvimnut, 134. 158 b. 293. 394. 421. 433.

125. Sekriniodlartotit, 189. 193. 229.

126. Ajornikka pivlugit kappiasukkuma 114. 374. 389 . 405. 412. 494. 554 .

127. Passigaksaungitotit, 105.

130. Okpertut ajugautitse 531 a.

132. Gûdde nakoridlarlavut (a), Kujagijartorlugolo (d), Nelliutilârpor illa (1), 17. 18. 47. 148. 157. 164 . 172h. 178. 181. 192. 196. 208. 223. 225. 234. 423 . 
Mel.

437. 456. 460. 596. 643. 756. 789. 803. 817. 836 . 837. 840.

136. Kuvianak Jêsusib ikkiminut, 319. 744. 833.

141. Ajortorsôvunga, 16. 219. 318. 380. 444. 462. 466. 575. 619. 667. 719. 786.

142. Inôtsib issua kaumaniojok, 432.

146. Jêsus tessiunga, 44. 73. 85. 142. 160. 165. 235. 243. 258. 284. 298. 339. 434. 471. 479. 572. 623. 645. 770. 774. 805. 827. 877.

147. Saugârsuk ikkilik, 152, 371.

149. Idluarsivoк ômat, 222. 482. 615. 637. 665. 839.

151. Niакок ikkilênar (a), Kanorle pijuksauvêk (g), Pivianartor Jêsus (i), 6. 10. 27. 30. 42. 75. 108. 119. 124. 132. 136. 143. 146. 172. 176. 262. 282. 313. 327. 370. 387. 403. 429. 477. 483. 507. 520. 528. 540.561. 592. 644. 655. 685. 693. 742 a. 759. 763. 772. 791. 824. 841.

152. Kristus piulijivut, 92.856 .

155. Tarminik pairsijotit, 60. 254. 311. 324. 342. 362. 467. 486. 524. 549. 555. 560. 717. 731 a. 736.745. 157. Illa kuviasukpunga, 45.

158. Pairкsijoк Gûdib Erninga, 19.

159. Attauser pijarialik, 42 e. 172 b. f. 217. 283. 296. 559. 584. 585. 605. 672. 694. 799. 879.

160. Gûb sullijangit nâmakput, 523 .

161. Piulijaumajosele, 286. 439. 545. 547. 597. 820.

164. Inôser illa nellâgut, 256. 266. 309. 328. 335. 341. 522. 595. 679. 683. 785. 801. 815. 829. 855. 864. 874. 882.

165. Jêsuse auggit ikkitit, 70. 76. 99. 109. 111. 112. 209. 232. 454. 499. 504. 505. 788. 846.

166. Saimanerub angininga, 71. 97. 155. 163. 234 a. 323. 346. 395. 445. 452. 495. 564. 582. 583. 590. 616. 682. 702. 721. 737. 738. 741.

167. Nâlegara okautsitit (a), Jêsusipta saimaninga (g), 7. 66. 138. 153. 25๊1. 300. 392. 417. 440. 458. 472 a. 514. 531. 551. 708. 865 a. 878. 
Mel.

168. Tussarnertunut nippinut, 13. 63. 65. 101. 113. 121. 226. 238. 348. 455. 804. 835 .

169. Tarnîk opigosudlarit, 40.

172. Jêsus ânanaujôvlune, 191.

173. Attauser pijarialik illitarilerlago, 329.

175. Kina tættigilârpigô, 845 .

182. Piulijiga pâmakpunga, 230. 604 .

183. NagliktoK Jêsuse saimaunga, 240. 280.

184. Aut illûnât ivlerinago (a), Illanârijama tarninga (b), 12. 41. 67. 86. 95. 115. 120. 130. 161. 304. 331. 400. 498. 566. 576. 588. 627. 634. 725. 753. 762. 767. 830 a. 863 .

185. Toкromut Jêsub naglikpâtigut, 26. 62. 87. 103. 118. 131. 135. 144. 156. 158 a. 162.177 .179 .283 a. 292. 317. 325. 381. 386. 388. 408. 492. 509. 556. 587. 626. 641. 656. 676. 690. 692. 700. 715. 726. 764. 828.

189. Jêsusiga oxititsainangatôK, 310. 533. 727. 735.

195. Gûd uvaptingnêpor, 351. 476. 580. 809.

199. Gûde sapputjauviksauvok, 529.

200. Jêsusiga naglingnernut, 427.

203. Nâlegar Gûde Anerner, 198.

205. Kejungme tokxogame, 315. 373. 535. 569. 709. 757. 858.

206. Nagliktakka piniarta, 15. 601. 714. 814.

208. Ajortorsôvogut (a) Piulimajose imgitsiaritse (d), 139. 141. 215. 347. 361. 497. 538. 565. 574.686. 814 a. 847. 860.

211. Tarne assiojuksauvok, 241. 818.

214. Tuksiartuksaungilangâ,, 185. 451. 516. 680.

215. Nelliutok кuvianak, 35. 38.54 a. 660.

216. Saugârsuk nangmalerame, 94 .

217. Jêsusima nâpkigivait, 214. 295. 398. 411. 510.

218. Naglingnersarijomavagit, 68. 98. 169. 180. 294. 308. 320. 333. 383. 453. 480. 537. 684. 746. 833 a. 848. 221. Gûdib naglingninga ittijovaksôvor, 9. 20. 268. 302 。 344. 349. 441. 473. 552 . 
Mel.

225. Nâlegar nêrtornar. 806.

227. Nâlegar nakorilavut, 808.

228. Hallêluja nâleklugo. 5. 149 a. 150. 159. 182. 255. 305. 356. 402. 464. 501. 712.

230. Kejungme tokkolauravit, 48. 61. 64. 168. 290. 316. 334: 367. 414. 426. 485 a. 532.581. 695. 734. 754 . 810. 823.833 b. 851.

232. Jêsuse nagligivagit, 415. 515.

234. Illûnarsoatik inuit, 260.

240. Jêsub inûvinga, 56. 128.

242. Nerriugviptingne tamaine, 618.

243. Jêsusib aunga kovijor pivluta, 122.

244. Takpaunga ârlorpunga, 91. 253. 297. 416. 769. 834. 249. Engelivaksuit, 800, 811.

310. SekKerngub nuilertub, 50.

376. Ômatiga tamaitalo pikka, 213. 271. 274.

520. Gûd nertorlavut, 542.

585 b. Nutarârsuk Jêsusivut, 42 b. c. 172 g.

595 a. Sillatujotuak, 42 d. 172 a. 814 b.

600. Attê tupaleritse okpertut, 42 a. 


\section{M GER UT IT \\ ATTOR AKSAT \\ ILLAGEKTUNUT \\ L ABR ADOREMETUNUT. \\ GÛDIB OKAUSINGINIK.}

1. Mel. 119.

Heiliger, :,: :,: Herr Zebaot 1.

1. Hailig Gûd, :,:

Nâlegar Gûd Zebaot!

Nunamiut, kilangmiut,

Illûnamassiatik

Gûdigivâtit, Jêsusib

Piulijangita perkutingat Angnerpâk. :;:

2. Gûdivut, :,:

Kujagitsainarlavut,

OKautsine tunningmagit

- Uvaptingnut, oKaudjivut

[ Gûde nangminer pivluta, Ajornivut pêjaromavlugit ToKkomut ailaungmet.

3. Illagêt :;:

Gûb taimak naglingmasse, Aungminut epкêjarluse
Tussatsainadlartaule:

Gûdib inûlidlarmasse

Tagva opigilavut illa!

Tamapta. :,:

4. Hailig Gûd, :,:

Nâlegar Gûd Zebaot!

Kaumanartut okautsitit,

Kaumaksartigilavut.

Gûde! mikserârtôgavit

OKautigitsainarlaptigit

Inungnut tamainut.

2. Mel. 58.

Gott! wie dein Name, so ist dein Ruhm. 2.

1. Gûd, attertut taimainivuttauk

Nertornarnît oKautsitillo, Tunnidlalauktatit

Illunaptingnut, 
Innôkovluta nâksaungitomut.

Kristusekut.

2. Inungnik ajokertuijose!

Oкаuseratsainalauritse :

Kristusib tamaita pingortimagit

Inuit assiolauktut ama Piulivait.

3. Gûb okausingine tamaine Окаutigijaujок Kristuse ; Gûdib Anerninga, okautsit pinginik,

Omattiptingnut pititsilaungmît

Illûnainik.

4. Kristus oKautigênarlavut! Pivlugo Gûb nâpkigingmattigut,

Aungallo kissime inuit tamaita

Ullapkutekartimagit $\quad \mathrm{Gu}-$ mut,

Tokrolaungmat.

5. Saimarsaijuksakatsangimat

Kristusib aungat assianik, Ajornivut ôma pêjalaungmagit;

Ômunga pingitut, illûnatik Piksaeroput.

6. Taimak idluarsinartôngmet
Illa, Jêsusib tokkunganut Illitarsigapta ajorniptingnik,

Gûbissumagijungnaimingmagit

Kristusekut.

7. Ôminga tussartoK, nâlegle; Ômunga pissiarijaugame! Imâglo erkaile: inûb attautsib

Inuit illûnaita piulivait Tokkominut.

\section{Mel. 84.}

Liebster Jesu, wir sind hier 3.

1. Jêsuse mânêpogut

Tussarlugit oKautsitit,

Sâtikit issumavut

Kilaub ajokertûtânut,

Omattit nunab pinginit

Illingnut kajungertikit.

2. Sillatunivut illa

Mattusimavut tâktomik,

Kaumartingipattigut

Kaumaksaijub Annernerpit.

Issumaloriksinermik

TunnitsivigittiguttôK.

3. Kaumajor nakudlartor Atâtamille pivotit Akiksulaungmittigut, Siutivut angmarlugit, 
Tuksiarnivut tamaita

Tussarlugit, saimartigut.

4. Tarnivut angmalerput Ajokertortaujomamut, Nâlekab Anerninga! Nâlegomadlaraptigit, Jêsub ajokertûsingit Omattiptingnut torkokit.

\section{Mel. 22 a.}

Ach bleib bei uns, Herr Jesu Christ. 4.

1. Jêsuse Krist, najortigut! Tâksiorkonnata sumut OKausernut illuptingne Kaumaksatsainartigulle.

2. Kiksarnartovingmênapta Sapputittigut tamapta; Uvaptingne okautsitit Sakramentillo pênarlit.

3. Omattipta tungavingat, Illagêktut tættevingat, Tagva Gûde okautsitit, Tungavigitsainarlavut.

4. Akkerartortut illingnik Okautsivit assinginilk Sakkertitsijut, agviakit, TammartitsilerKonnagit.

5. Nâlegar Jêsus kissivit Nertoræksaujotôgavit;
Taimaimalle sapputikit

Tættekangitut asșingnik.

\section{Mel. 228.}

Das Wort des Herrn ist recht. 5.

Gûdib orausingit illa,

Idluarsitigivakka,

NekkoKsitigigapkit

Sæglotuitoksôgivut

Piloringnarput uvamnut,

Mallitsainarupkille;

Tâpkoa, illa,

Apkutauvut - Gûd illingnut,

Nâlekupkit

Tarnib serrivigidlarpait.

\section{Mel. 151i.}

Sein Wort ist wohl gegründet. ${ }^{*}$.

1. Gûdipta orausingit Tunganarmarikput; Angerningit illûnatik Nellâgôtsainarput; Sappernartokangimat Ômunga, Gûdipta Attinga hailigeongmet; Nertorlavut tâmna.

2. Gûmut pititaumajut Aglait illûnatik, Attornadlarput illa, Ajocertûtsemut ; Kaujititaukovluta 
Tâpkonunga mâne, KanoK illingavluta Kilangmut pigapta.

3. Kaikojomangmattigut Ernermigut illa, Piloringnartovingmut, Tamanna pivlugo Aglangnut makkonunga Innerterpâtigut Sakkertokaleranget Nangiarnartomik.

4. Tammaisaraidlarapta. Aprosininganik, Tâpरoa овausingita Kaibladlarpâtigut Ârigasuarluta Torârtilutalo Sapputsijungnartomut Uvaptingnik mâne.

5. Aglait hailigit illa Taimak illingavut, Âkiktaudlarkovluta Tâpkonunga mâne; Inoguijauvluta Nâmagijanganut Pivalliarovluta Idluarsinerme.

\section{\%. Mel. 167.}

Herr dein Wort die edle Gabe. 6. 1. Nâlegara orautsitit Piksaritsainarlakka,
Angnerpâkutig igapkit

Nunab pinginit illa; Oкаutsitit pingikupkit, Tættiksara nanêkâ?

Okpernermut oKautsitit Tættituarivakka.

2. Hallêluja Amen illa, Ikajunga Jêsuse!

Omattipkut nâleklanga

Okausernik tamaine; Kajungersadlalaungminga Mâne kivgartorlutit, Mariatut ingilanga Sângnut nâleklutillo.

8. Mel. 68.

Gottes Wort ist klar 7 .

1. Gûb okausinga Imaipor illa: Inuille nunamiut Jêsub tokrotigingmagit, Sâgiarkovait, piulingmagit.

2. Gûb naglingninga Kiblingnadlarmat, Tâpsoma okausinginik Tussâjut nâletsiartullo Kibliktaulerput ômattimikut.

3. Taimailungipat Gûb kivgangita Okumaitiginajarpât, Inuit sâgiarkolugit; 
Gûb okausinga, kiblêjo K illa.

\section{Mel. 221.}

Dein $W$ ort ist ja die rechte Lehr. 8 .

1. Okautsitit illa, ajokertûsiovut

Nellâgôrtomik inungnut, Kaumaniovullo raumaksaitsainartok

Uvaptingnik inôtseme;

Sappûlutaksauvut sorsungnerme,

Missuksoangovut nekkoKsinartok,

IpteKartitsijor tarniptingnik illa,

Kannimasiptingnik ikpigigaigupta.

2. Anernît ajunginek Nâlegak Gûde

Tunnidlalauruk uvamnut, Nakkiterkovlugo illumne tamainik

OKautsivit tukkinginik;

Kemergokovlunga tâpkonane

Uvamnik tamâne inôtseme;

Illitaritsainarkovlunga

uvamnik,

Tammarmerkonnanga ajoKertûsernit.
10. Mel. $151 \mathrm{i}$.

Lass mich dein sein und bleiben. 9 .

Perkutautsainarlanga

Illingnut Gûdiga!

Illingnit pêrtaunanga

Sumullônêt illa;

Sokkosertailitinga

Ajokertûserne!

Tagva nakorivagit

Issokangitomut.

\section{Mel. 16.}

Ieh will bei der Lehre bleiben 13 .

1. Gûdib ajokertûsingit Nâlegomadlarpakka Okauserlo kiblingnartor Ômamiutarilugo.

2. OKausernik ômattiga Missigijarangame, Miksexârninganik tagva Nagvârpunga illumne.

3. Okalaurit Nâlagara, Nâlegomadlarapkit, Okausernik okperama Tuppaktinamga igvit.

4. Nertorlagit nâleklagit, Nellâgôrtilaungminga Jêsusiga okautsitit Pituaridlarlakka.

5. lllumne kaumaksalaunga 
Sulle tâ.zsioruma; Anerner tussalaungminga, Tessiortigigapkit.

6. Nutakratut pitiminga Atâtak! perkojarnik Inôsimne nâleklunga, Ungagitsainarlagit.

7. Atâtapta isumangit Nâpkiniktôjut illa! Naglingnermut uvaptingnik

Sokrosijuitôvut.

8. Illûnamnik pitipagit,

Ajoralloartunga, Inôvigijomavagit, Jesusiga, piginga!

\section{Mel. 184a.}

Erhalt Herr Jesu uns den Sinn. *Anh. 2.

1. Nâlegar Jêsus-orausernut Sokrosijuitittigut!

Ajokertûtsivit tukkinga Kaujijaule uvaptingnut; Piniarnitit malliklugit Arvertatsainartitittigut, Tammaraigupta tâpkonangat

Kaujitisâlaungmittigut.

2. Orautsille tokrotaunernik Tamainiglo âniarnik,
Uvaptingne nutautsainarlit,

Nekkorsinartotôngmatta; Kappianartune tamaine Tarniptalo timiptalo Pitsartutigitsainarmagit, Mannigortigilugillo.

\section{Mel. 168.}

Nic htskann armen Menschenherzen. $* 22$.

1. Ajortut ômattinginut AngnerpâKatsængilak, Okâlanermit Jêsusib Aniadlarninginik, Tokkunganiglo pivluta; Pilloreutiksaptingnik Âkiksoilaurame Assiolerkonnata.

2. Jêsub piuliklerninga Itterutigivavut Kuvianartovingmulle; Siorniudlarnivut Merngortûtigiguptigik, Tagva ânianingita Kappijaudlarningallo Mannigudlarpâtigut.

\section{Mel. $115 \mathrm{~b}$.}

Wie wohl ist mir, wenn mich mein Hirte führet. ${ }^{*} 23$.

Tessiorluarpânga pairijima 
Nerritilunga oKautsiminik;

Kaumartilunga missigitipânga

Ajulertut nagligigamigit; Tarniga nekkoKsitipâa Kilangme kaujimajæksaKartipânga.

\section{Mel. 206.}

1. Nagliktakka :,: Piniarta
Nâlegavut - Nakorid :;: larlugo!

Kovidlalaungmago:,: aune, Avatine, sennerane

Naglingnermut ;;: ikkilerkongmagit.

2. Mariatut :,: ittiggangnut Ingilanga - Orautsil :,: lo mânna

Nâletsiarlugo, :,: illa

Ômattiga kibliklugo Okausernut ::: tussarnidlartunut. :;:

\section{INUIT KATTAKSIMANINGAENNIK KRISTUSEMULLO} PIULIJAUNING ENNIK.

16. Mel. 141.

O wir armen Sünder. $* 28$.

1 Ajortorsôvluta

Inûlertogut,

Ajortullinipta,

Pitipâtigut

Kappianartomut,

TokKotiluta

Issorangitomut,

Tamapta illa.

2. Asserorniptingnit

Piuliluta

Nangminer uvagut

Sappidlartogut;

Pitsartojoksôngmet

Ajorner; Gûde
Pivluta tоккоуок Anialune.

3. Kristuse nuname Inûlingipat, Attilugo auvut, Tokkolaungipat Pivluta tamapta, Nâksaungitomut Tammarajartogut, Assioluta.

4. Taimaimat, tamapta

Kujagilavut

Gûb pingasôlijub

Amungmattigut

Kappianartomit ;

Kristus pivluta. 
Tokrungmet, aungminut Piulivluta.

5. Atâtaujub sorlo Gûb saimanermik, Taimak pivâtigut

Akkekangitomik Ernermigut; tâmna Pingmat tokkomut Pilorerkovluta Nâksaungitomut.

6. Toккоmut Kristuse Naglikaptigut, Pivluta kejungme Siorniornernut; Kujagivaptigit Nâksaungitomut; Najutsainarlutit, Ikkajortigut

\section{Mel. 132 a.}

Nun freut euch lieben Christeng'mein. 16.

1. Illagêt næglingnartose! Kuviasukta, Gûde Nakoridlarlavut mânna Nekkorsitimmatigut; Piniarningit pivluta Tattamnadlarmatta illa, Akkitudlarmattalo.

2. Kellaksorpânga Sâtanib, Ômame tokkojunga, Kaut tamâllo ajornerit Kappiasuktipânga,
Kallerêktiluarpakka Ajungitokatsennanga Ajortoliksôvunga.

3. Ajungitullinikkalo Asserorsimangmatta; Illunât pijomanera Gûdemut ôminarpor; Kappiaktoadlarpunga Toккоnartomut pigama, Tâktomut kivilunga.

4. Gûdib kattaksimanera Illapsugosungnermut Tækkogamiugie, tagva Ikkajortaukovânga; Atâtatun ômamine Sêkpor sorlo, næglikame, Pituaminik pivânga.

5. Pivor imâk Ernerminut: „Erdligijatuara „Piulijartorniaruk! „Nâpkiginartor taimna, "Amuaruk ajortunit, "Pivlugole tokrolutit, „Inôtiuk illingnut.“

6. Ernerub Atâta nâlekpâ Tikkilunga nunamut, Uigasukut pivlune, Illa ivsornaitokut, Uvamnut kattangutaulerpok

Akluvlune arvertarpok, Sâtanas kellaksorpâ. 


\section{-}

7. OKaupânga : ungaginga,

Piulijomagapkit,

Pivlutillo toккоgama

Pitlaraksaukonnægle ;

Pigigamga, pigigapkit,

Najornimnêkuvit tokkub

Avitingilâtiguk.

8. Nunamut kovivât auga,

Kejungme tokkolunga,

Pivlutit âniajunga,

Tagva okperiginga.

Innôtsima tokKo êva,

Ajornitit pêjarpakka,

KuviasuKovlutit.

9. Atâtamnut paungarpunga Itterlunga kilangmut Pivlutit tuksiarlunga, Anernimnik pilutit; Taipsoma mannigorpâtit Sumiglônêt kiksaruvit, Tessiutsainarpâtit.

10. OKautsikka malliktakit

OKautigilugillo.

Nâlegaunimnik oKarit, Angijororkovlugo;

Inuit nellangainingit

Illûnaita allarlugit;

Taimailiorkovagit.

\section{Mel. 132 a.}

Ach Gott, es hat mich ganz verderbt. 17.

1. Gûd sângne sakKersimavOK
Asserorsimannera,

Uvamnut Adamit pijor;

Idluitorsôgama,

Kiale piulivângâ?

Ajornikka angimatta

UnnuktoKsôvlutiglo.

2. Jêsuse aivigivagit

Kakkialidlarlunga;

Ômamut tuksiarpagit;

Piuliuk tarniga

Âniaksakka pêjakit;

Idluinikka pivlugit

NungutaulerKonnanga.

3. Tuksiarviksatôvotit, Assingnik nellovunga,

Piuliklertetôgavit

TokKungnut kelluvarma;

Aungnullo inûlivarma

Ajornimnit, Gûb sângane.

Sakkerungnarkovlunga.

4. Jêsuse Kaujimavotit

Kappiasudlarnimnik, Mattominga pêjailaurit,

Kiksarnimnik tamainik;

Nâlegar tættigivagit Jêsumik atteKaravit, Sapkoniængilarma.

\section{Mel. 158.}

Der edle Hirte, Gottes Sohn: 18.

1. Pairsijok Gûdib erninga Nagliktotôgame, Nâlegaunine Kemakpâ Inûlerlunelo, 
Tammartok saugakotine

Kenneriartorlugo,

Tammarsimangmat nuname,

Uttertitsomavlugo.

2. Saugârsugle tâmnauvotit, Tarnîk okperiuk!

Pivlutit nerchevingmêpor, Illuvermêporlo:

Perkutinganut tunnigit, Pivlutit toккоlaungmat, Kujagijartoleruglo Inôtsiarnernulle.

\section{Mel. 221.}

$\mathrm{Du}$ ewiger Abgrund der seligen Liebe. 20.

1. Gûdib naglingninga ittijovaksôvok!

Kristusekut sakкijârtoK, Ikkomatun-îtor kajungersaininga

Ipaksijaujungnangilar;

KanoK næglikangne ajortuggit?

Nâpkigigangne ômigijitit? Illa, igvit illanâkadlartotille

Ajortoliksuit annerilaurangne.

2. Ânanaunersoar Nâlegak Gûdiga!

Nâpkiningnît angnerpauVOK;
Tuksiarniptingnit pitiluarluta,

Pitlaræksaugalloartogut; Atâtavut tagga tættigivavut,

Kippiniarlutalo Ernermut, Anernermut ajokertortauvlutalo;

Tuksiarniptingnik pititaugaptalo.

\section{Mel. 22.}

Lord I am vile, conceiv'd in sin. 28.

1. Nâlegar ivsornartunga Ajortoling nit pivunga, Pigamalo Adamemit, Ajortomut kattaktomit.

2. Annersâtainarniptingnit Tоккоmut illingajogut; Idluartut perкojattit Ingergapta ajorpavut.

3. Ômattipkulle Gûdiga! Nutâmik pingortiminga, TækkoKovlunga tarnip kut Nangiarama tâktomut.

4. Gûde sângnut pâmakpunga,

Nâpkiningnît tættigilugo, Idluarkôrumalônêt Illupkut ivsornartunga.

5. Pingmat pidlaræksaunera 
SaimauteKatsengilanga, Illûnara Gûb sângane Merngoêrviksakangilak.

6. Aggut Jêsuse Gûdiga! Epkêjautiksarivara, Ullapkoserlunga Gûmut, Pitsartojoksôngmat aggut.

7. Gûdiga tuksiarpagit Piulinga ajornimnit, Ômattiga serkomitok Nutâmik inûlingmiuk.

\section{Mel. 14.}

I with the fallen human race. 27.

1. Inuit kattaksimajut

PeKattigivakka,

Tokkovlunga ajornimnut Kappiasukpunga.

2. Nagligosulktub Jềsusib Mânna tækkungmanga, Avisimanera Gûmit Illapsugidlarpâ.

3. Ungamut naglikaminga Nâpkoarivânga,
Ikkajoromagaminga

Pivânga: makkitit.

4. Ajornimnit ubvarmanga

Pêrlugit aungminut;

Idluarsitilerpânga

Pitilunga Gûmut.

5. Pitlaræksaunek pêrmago

Erksijungnaipunga,

Erkartungimanga; aunga

Pingmat nâpkinermik.

6. Ikkomamit Sâtanemit

Gûdib amuvânga, Ikkinginêlunga, sumut Kiksartitaunanga.

7. Kikkiaktortaunerminut Kellarudsârmanga, Nâleklugo unganermut Malligomavara.

8. Tarnîk kuviasudlarit Jêsusib Kristusib Aungminut piulingmattit, Ômamut nertoruk?

\section{RRISTUSIB INÛLERNINGANIK UVININGMELO}

\section{SAKKERNINGANIK.}

\section{2อి. Mel. 22 b.}

Von Adarn her war'n wir verlor'n. *38.

1. Adamekut tammarapta, Pivavut Gûb ningausinga
Tokromut tarne timelo

Ajornerub killermagik.

2. Kivgauluta ajortomut Kattangnerapta torkomut, 
Kappianartomêluta

Piulijiksakarata.

3. Gûb tækkonârpait inuit Kennerlune nâlektomik; Adsigêktulle illûnatik Uvinetuinaungmatta.

4. Nellâgut ivsornaitomik. Sângane idluartomik; Nagvângilak; illûnatik Adamut kittorngaungmatta.

5. Taimak asserorsimanek Gûdib tækkomagamiuk, PiulijiksaKaratik,

Inuit piulilugit,

6. Naglingningat erkaitipâ OKautsine nellaudlugo; Okarame illapsumut: Ikkajorlakka inuit.

7. Pingmijok : Nâpkigilakka. Tunnilagole Ernera; Ôma sillaksoarmiut Tokкomit piulilugit.

8. Abrahamut angilaukpok, Davidemullo pigivok, Tunnijomârmago Erne, Sillarsûb inunginulle.

9. Nellautaijunullo pirok, Taipkutigûna kaujijauv.or;
Taimaimat itsaksoarnit, Okpertut nerriugivât.

10. Taipkoale kippilertut Jêsusible sivorngagut; Nekkoksivut tækkonnago Kristus okpervigivlugo.

\section{Piviksanga nelliumat}

Jakoblo nellautainingat: Uigasuk Gûdib tagva Ernijuksar annerivâ.

12. Taipsoma illuane Gûb Âxilerpâ nutarârsuk, Pitsartunerminut illa; Tâpsomûna nâp kigiluta.

13. Kristus ivsornaitôjotit

Pivluta inûleravit, Ivsornaitilaungmittigut! Pêjarlugit ajornivut.

\section{Mel. 70.}

Der Erstgeborne. 23.

1. Erniangokârto K Nuname sakkerpor, Uvaptingnulle Angerutaulaurtok, SakKêdlarpok nellonartunik.

Sivorngane Kaujijaungitunik.

2. Ajorniptingnut Tokkungadlarapta; 
Piulijaunek

Ajudlarlugolo;

Ômungalo kajungerata,

NaglingniktoK Kennuvigi-

nago.

3. Jêsus okarpok:

„Ahâk! aggerpunga,

„Nagligosumut

„Inuk simmerpara,

"Toкkoniarlunga pivlugo,

"Gûdib ningausinga ramilugo.“

4. Piuliklertor

Taimak pitlartaujok,

Ingmik tunningmat

Kikkiaktortaunermut;

Mânna Gûb kittorngarilerpait

Ajornermaingnik sænnikterijut.

5. Nâpkiningnermut

Piulijauvogut;

Kaikojauvluta

Jêsus nivingangmat;

Piluta serrinartovingmik, Issuanit pijarêlauntomik.

\section{Mel. $11 \mathrm{~d}$.}

Nun komm der Heiden Heiland. 24.

1. Nellojut $\widehat{\text { piulijingat }}$ Kailertor uvaptingnut
Gûmit uigasukut

Tattaminiarivavut.

2. Pingimallo angumit

Gûble anerninganit

Gûb inûlertub ôma

Apkotine attorpâ.

3. Iniminit annijok,

Ânanauner Kemmakpâ, Atâtamit pigame, Ama utterivlune.

4. Atâtatun-ipotit,

Ajugakanginavit Ânertamut timingnut Serngnigikit timivut.

\section{Mel. 185.}

0 verehrungswürdige Minute. 25. Opinartotôjok uvlox taimna! Uvlok nellekangitor:

Uviningme Gûdib sakkervia Nikkanarsârvialo;

Uviniolermat nutakKatut, Piulijomavluta, uvagut Assiojuksaudlartut, Pissivaille aungminut.

\section{Mel. 151a.}

Auf auf, weil der Tag erschienen. 26.

1. Attêtôk makkilerta! Uvlok sakkidlarmat Kuviasuktitivut 
Piulijaunartoк:

Attêle makkilerta!

Jâre tikkidlarmat

Atâtat nerriugijangat

Kippiniarnermut.

2. Attannît unnudlartut

Tækkojomajangat

Nellautaijullo illa,

Tussugilauktangat,

Sakkersimavok mânna,

Tamanna pivlugo,

Nelliunek tamanna

Kuvianadlarpoк.

3. Tamânêpok, Atâtab

Pijomajanganik

Nâmaksititsivlune

Nâletsiarnermut.

Aulingmut tokkominut

Kassilinartomut

Piulikleromavok

Assiolauktunnik.

4. Akkisartuivlune

Uvap tingnik : illa,

Tokкotaujomalaukpok

Senningajolingme,

Tokkungat tippinganut

Pêjartaukovdlugo

Ôminaut ingergapta

Kollangiartavut.

\section{Mel. 22 b.}

Lob sei dem allmächtigen Gott. 27.

1. Gûde ajugakangitor Nertorlavut, Ernerminik
Tilliklermat uvaptingnut

Taimak nâpkigingmattigut.

2. Piulikterteokovlugo

Uvaptingnik, ajornivut Pêjarlugit, saimanermut Aitiluta Kaumajomut.

3. Naglingner nâpkiningnerlo

- Angijovaksôvuk illa;

Gûb taimak piniarmattigut

Kujagitsainarlavuttôk.

4. Pingortitsijub tamainik Inônivut attilerpâ, Arnab timâ nachoginago Tamna inûlervigivâ.

5. OKauseк Gûd nâksaungitok

Ivsornailune inûlerpox; Gûd kiglilervikangitok, KiksarnartovingmutaivoK.

6. Inugle suksauvâ? tâmna Pivlugo, Gûb tunningmago Ernine; suna pivlugo Taimak piniarpâtigût?

7. Kappianamêk inuit Nachutsainartut illingnik, Kamangimut Jêsusemik, Gûb ningarijæksaringmagit. 
8. Ômât sôgle sillaidlarkit? Gûdit aiviginginangne, Tâmna pairijomavlutit Kaivok nikkanarsârlune.

9. Uvlome illallioruk, Ômattit tunnilerlugo, Itterkovlugo illingnut, Nekkorsijaujutiksarnut.

10. Tikkerkârame nunamut Sinnaungasuidlalaukpoк; Tikkimikpât okpingitunut Kappianadlaromârpoк.

11. Okpertulle Kristusemut, Nelliutikpat, itterput Kilangmut serrinarvingmut,

Engelillo najugænnut.

12. Atâtavut Kotsingnerme Erningalo Ânernerlo Nertortaule sorairane Kujagitsainarlavullo.

\section{Mel. $11 \mathrm{~d}$.}

Gott sei Dank in aller Welt. 28.

1. Gûd nakoridlartaule Mânna nellautsimangmat, Ernine tillingmago Mannigoromavluta.

2. Sivorlipta $\overline{\text { uttakkivât }}$ Nerriugitsainarpât,
Tikkimat okausinga

Nellâgut nellaudlarpâ.

3. Kujagivagit tagva,

Piulijartoramga!

Ômattiga illingnut

Inniksautilauruktôk!

4. Tâpsomunga itterit,

Pigigangne kissivit,

Ubvalaungmiuk aungnut

Ajortunit tamainit.

5. Mannigolaungmingale

Nellopкotidlarama,

Nellâgulle illingnut

UngatsainarKovlunga.

6. Inôgutetôgavit, Ama tikkileruvit Ajorungnailungalo, Pagrearlagit tagva.

\section{Mel. $151 \mathrm{~g}$.}

Wie soll ich dich empfangen. 29 .

1. Kannorle pijuksauvik Illalliorlutit?

Erdligijatuara

Tikkidlaleravit!

Tarnima tuksiarmut

Nakoridlarlitit,

Ungagitsainarlagit

Uvlut nungulugit.

2. Ânianadlartomut Jêsuse aivotit, 
Ajornikka pivlugit Ânialauravit.

Tâktoksoarmêtunga

Sâtanamêlunga,

Piulijartoramga

Opiguktiparma.

3. Parngnanaerutsaujunga,

Annitilerparma,

Kaujitilerlungalo

Pigijomagamga;

Ajornikka tamaita

Illingnut pivattit,

Piloridlarkovlunga

Ânialauknotit.

4. Naglingnivit kissime
Akкartilaukpâtit

Tamaunga nunamulle,

Kilak remmaklugo.

Asserorsimangmatta

Sillaksoarmiut,

Piulijomavluta

Tikkilerpaptigut.

5. Ajornise pivlugit Sænnikterijose!

Erkarsiuk Jêsusib

Nagdligidlarmasse;

Takopsoagimullo

Inûlerlunele,

Piulijartorpâse

Pilorerkovluse.

JESUSIB KRISTUSIB ERNIANGONINGANIK ARVERTARNINGANIGLO NUNAME.

\section{Mel. 22 b.}

Vom Himmel kam der Engel Schar. 30.

1. Engelit kilangmit pijut Sakkerpait mianersijut, OKaulugit: NutakKamik Nerchevingme-ilertomik.

2. Betlehemele Dâvidib Igluge senginêtomik; Michamut orautaujomik; Nâlegak Jêsus Kristusemik.
3. Ôminga kuचiasugitse! Gûdib illagilermasse, Uvinipsingnit aupsingnit Erniangongmat kattangut.

4. Mânna ajornerub tokkub Akkonartipâse kanok? Ômisuktullo tamaita Gûub sâlagingmagit illa.

5. Allaromanginivâse Tættigilugo pititse; Sorsugomajokalerpat Illipsingnut, sullingilat. 
6. Tamainut sakkeromârmat Pingmasse Gûdib Erningat;

Taimaimalle sorairase Kujagidlarsiuk Gûde.

\section{Mel. $22 \mathrm{~b}$.}

Euch ist ein Kindlein heut geborn. 31.

1. Uvlome erniangojor Uigasungmit nutarak, Kristus Nâlegax Gûdivut Piulivluta tamainit.

2. Piloringniksat tamaita, Gûdipta tunnergutingit Pigivavut sorairata; Kilangme inôKovluta.

3. Nellunaikutarissiuk, Nerchevingme nutarârsuk Nagvârpâsse illimajok; Sillamiglo aulatsijoK.

4. Ôminga Kuviasukta! Ailerlutalo tagvunga, Nautsertırlugolo Gûdib Pingmattigut Ernerminik.

5. Ômattik ullernairuktôk, Nerchevingmêtorle kinâ? Nutarârsuk ânanaujok Illa, Jêsuse nagliktor!

6. Igvit salutitaulerit!
Ajortunik nachongitotit, Nagliklunga tikkinamga Kappianartomut maunga.

7. Pingortitsijok tamainik Kanok taimak aklulerKît?

Inangajotit ivingne, Nergjutit nerkiksangine.

8. Iviksukanêlertotit, Imutit annorâvinît; Tâpkoa piksatuatit, Nâlegavitôjotille.

9. Taimaglo idluarivat Kaujitilunga nellâgut Nunab pingita nelîta Sunaungininginik sângne.

10. Nægliktigalo Jêsuse!

Innerarit ômattimne, Takkamanêtsainarlutit Puigorkonnanga illingnik.

\section{Mel. $22 \mathrm{~b}$.}

Christum wir sollen loben schon. ${ }^{*} 55$.

1. Kristus Marîab erninga Nertudlarpavulle mânna, Sekkerngub nippininganut,

Sillaksûblo issuanut.

2. Tamainik pingortitsijok, Nunamele inûlertor; 
Uviningminut inuit Ajorungnaidlarkovlugit.

3. Tâmnale inûlingipat, Illûnarsoatik inuit Assionajalauktulle Issorangitorsoar'mut.

4. Taimak kuviasukpogut, Jesus nutarârsûlermat; Inûvingminit kaitsingmat Ullapirsautiksaptingnik.

\section{Mel. 50.}

Gelobet seist du, Jesu Christ. 32 .

1. Jêsuse nakudlarpotit Nuname inûleravit Illale uigasungmit, Kuviagivat Engelit, Hallêluja!

2. Issokangitoк каumajoк Inungnut nuisimavok, Kaumavlunelo tâktomut, Kittorngautilerpâtigut Kaumajomut.

3. Atâtab issokangitub Ernetua nagvârpavut Nerchevingme, uvinivut Auvullo attimangmagik Hallêluja!

4. Sillaksûb pingortitinga Marîable sardliarpâ
Tâmna nutarârsôlaukpor Tamainiglo aulatsijor. Hallêluja!

5. Atâtab Erninga Gûde Tujormiojor nuname; Kiksarnartomit pêrluta Kilak kingormgutilugo. Hallêluja!

6. Aklulerlune tikkipor, Naiperkotigingmattigut; Ajornivut pêjarlugit, Engeletun-erKovluta. Hallêluja!

7. Tagva tamât pivâtigut, Nagligilauramittigut; Tamanna kuviagilavut Opigitsainarlavullo. Hallêluja!

\section{Mel. 215.}

Die Zeit ist uns ganz freudenreich. 33.

Nelliutok Kuvianak!

Nertorlugo Gûb akka, Kristus kilangmit akkarmat Nunamut tikkiluta; Nellekangitok nâpkineк Gûd uviningmik attingmat, Inûlertor pivluta, Tamainik adsigivluta, Ajornerle piginago; Salutipagit, Amen. 
36. Mel. $22 \mathrm{~b}$.

Wer kann's dem Vater g'nug verdank'n. " 58 .

1. Kujagivlugo Atâta Nâmaktomik pijor kina? Ernerminik pingmattigut Inûlivlugit tarnivut.

2. Idluarniptingnuungitor, Nâpkigosungnerminulle Illapsugilerpâtigut; Kristusemik pingmattigut.

3. Tâmna nutaraunerminit ÔksivoK akluniptingnik; Pivlutatauk niakoa Innangavekatsennane.

4. Inônermine nuname Jârille drei und dreisiggit,

Piksaminik pekangitor. Inôkovluta tokKovok.

5. Piulijivut Jêsuse!

Erniangojotit mâne, Ivsornainerme Adamekut Assiojome uvaptingnut;

6. Ivsornainît idluarnîllo Saimanermut tunnikiktôk, Illûnaptingnut, tarnivut Inûlijaulerkovlugit.

\section{3\%. Mel. $14 \mathrm{~b}$.}

Lobt Gott ihr Christen allzugleich. 34.

1. Illagêkluta mânna Gûd
Nakoridlarlavut!

Ernerminik tilliklermet, Piulidlarluta.

2. Jêsusipta kiglinganit Nagligingmattigut

Illungertortomut ômamut Nagligidlarlavut.

3. Atâtab sânganit pivok Nutaraulunelo;

Nerchevingmelo nellavor Annorâkarane.

4. Kivgaulerpor, inukulluk Nâlegaukovlugo;

Illa angijovaksôvor Illænâkarninga.

5. Uvlome apkotiksavut Kilangmut angmarpâ; Ingminut Kaikungmattigut NellipsarlavuttôK!

\section{Mel. 215.}

Ein Kindelein so löbelich. 35. Nutarârsuk ussornartoK Inûlerpok pivluta, Uigasû̉b ivsornaitub Nuname erniarivâ; Tâmnale inûlingipat Assionajalauktogut, Piulivâtigulle;

Jêsus! Kujagivaptigit Pivluta inûleravit Aungnut piulivluta. 
erniangoninganik

39. Mel. $22 \mathrm{~b}$.

Freut euch, ihr Menschenkinder all'. ${ }^{*} 61$.

1. Illûnata opigukta! Ahâk, Marîab erninga, Tagva Jêsuse Gûdivut Tikkidlartor uvaptingnut.

2. Kattangutigigaptigo; Opigukta nertorlugo; Inûlermat uvaptitut, Gûd nâlegaujoK tamainut.

3. Serrinartut tikkiupait,

Kiksartut kugvingit pêrpait,

Nekkorsivait inûlermat, Aunârdlunelo tokKungmat.

4. Gû̉b Ernine naglingnermut,

Pitimago uvaptingnut, Kanok ajunginersamik Pinajarpâtigut sumîk?

5. Ajornivut pêjarmagit Gûb, erkaumajungnailugit Pivlugit piniartavut, Erkartortaujungnaitogut.

6. Piulijautigivavut, Itterlutalo kilangmut; Ilkkajortigut Jêsuse, Tættigênarlaptigille.

\section{Mel. 169.}

Ermuntre dich mein schwacher Geist. 36.

1. Tarnîk opigosudlarit, Atâtamik taijaujub Nutarârsûb tikkimmatit; Serrimut erkileruk! Unnuar tamædsa pingmat, Uviniptingnik attingmat Pivluta; tokkominut Piulidlarpâtigut.

2. Jêsus salutitaulerit! Nâlegatôgaville, Gûdib saugârsugingmattit, Kujagidlarpagille ; Illûnamnik pitipagit Piginga! ungagivagit, Piulilauraptigut Uvagut, tammartogut.

3. Inukotitit Jêsuse, Nachonartorsôlauktut, Igvit toKkotigigangne Nutâmik pissivattit. Ukkoa sinnaunganartut Ôminadlartorsôlaukput, Tâpkoale pivlugit, Kilangmik Kemaivotit.

4. Nutarârsuk Jêsusiga! Nâpkigosudlaravit, Nagliktor Kattangutiga, Illumnik kiblêjotit; Naglingnermut ungalunga Illingnullo inôlanga, 

Ômattiga nuname
Innigilaungmiugle.

5. Jêsus pituarijagit, Inôgutigivagit

Sumik kuviasuktitiga

Tunnitsivigilagît?

Timigalo tarnigalo

Anerneralo pikille;

Illuêngardlunga mânna

Pigitsainarparmale.

6. Jêsuse nakudlartotit!

Inungûtigigamga;

Aglât ajornikka aungnut Pếjarangne tamaita; Naglingniktotôgaville, Igvit ikkajulaungminga Nuname kilangmelo Nertoromadlarapkit.

\section{Mel. 184 a.}

Freuet euch ihr Christen alle. 37.

1. Okpertose tamapse mânna Kuviasudlaleritse!

Gûdipta angijomik illa

Piniarvigingmattigut.

Illale kuvianadlarmêk!

Kattangutigigaptigo.

Kristus, agvairtok kiksarnartunik

Kaumaksaijor tarniptingnik.

2. Jêsuse kannok nâmaktomik
Kujagijæksarivagit?

Kigligiudsivunga igvit,

Pilorigutigigapkit:

Taimaimat sapkotailitinga

Illingnit nunamênimne,

Ômattiga ullapirsaumik

Ajoksarniarkonnago.

\section{Mel. 151g.}

Anbetungswürdig's Kindelein.

1. Nutarârsuk Jêsuse!

Pâmakvigivlutit

Tuksiartogut sângne

Nellopкotinermut;

Kujagidlarpaptigit

Assioliurapta

Kugvinut nertorlagit,

Tikkinavit mânna.

2. Kưvianamêk uvlome

Gûb pingortitsijub,

Inûlernerminulle

Illagingmattigut;

Piulingmattigulle

Kattaksimaniptingnit;

Piulijomangmagit

Tamaita innuit.

3. Gûde nutaraujotit,

Pillitaulangatồ

Mikkijôlungitomik:

Illingnik pilanga.

Inûlernît Jêsuse

Perkutigilago; 
Piulijaukovlunga

Ajorniâlumnit.

\section{Mel. 600.}

Christians awake, salute the happy morn. 1261.

1. Attê! tuppaleritse okpertut,

Uvlome Jêsus kaingmat nunamut!

Naglingneк okautaujungnangitoK

Pâmangavlus e opigisiuk! Kuvianak Jêsub inûvia, Gûb uigasûblo erningata!

2. Tagga, Engele pângat akkartor

Mianersijunut okâlavok:

„Tussaritse kuvianartomik

„,Illûnainut pilârniartomik!

„Nunamut pivok Gûdib erninga

„Mânna inôvlune! Hallêluja!‘6

3. Kilangmiogasæksuit tagva Gûmik nertordlerput:

„Hallêluja!

„Ullapirsautekarle nuname

„Kuvianartokarle inungne! 6
Kaumajokarungnaerivok ama,

Paunga aisârdlutik nippangmatta.

4. Uvaguttauk inukullôjogut Illagilavuttôk nertordlertut!

Mâne soraerata nertordlerta,

Takpaungakilangmut perKârata!

Takpânelo issokangitomut Tussartautsainarlit imgernivut!

5. Illa Jêsusiga nagliktara Ômatiga illingnut kaipara. Unêt sujudlartok sangêdlune

Nertornautiksarnut âkiktaule!

Taimaimat, Jês us, tattamnartotit,

Uvlome kait uvamnut, kailaurit!

\section{Mel. 585b.}

Hail thou wondrous infant stranger. 49.

1. Nutarârsuk Jêsusivut

Tujormiangovlutit

Piulijomagaptigut

Nunamut tikkipotit.

Nertortaugit, :,:

Gûd issorangitotit! 
2. Kilangmit nachoginata Pivor Gûdiviksoak. Annerimanivut illa Ipaksinatsangilak. Tattamnarmêk, :,: Gûte inungortitak!

3. Paradîsib perkutingit Assiojilauktavut Tagva nutakkab kaitangit! Ama pigigivavut! Opigukta, :,:

Ômamut nertorlavut!

\section{2c. Mel. 585b.}

Angels from the realms of glory. 51.

1. Engelit pitsartujose Serrekattaudlaritse! Gûmik kivgartudlartose Ama akpârmititse!

Pâmagitse :,: Nutarârsûb sânganut!

2. Nerriugidlartamingnik Pivut mianersijut; Mallikput uvloriamik Tagga illisimajut; Pâmakterput :,: Nutarârsûb sânganut.

3. Ajortoselo illipse Kingurainiarase! Kaititse, piulijipse Saimarvigidlarmase. Pâmagitse :,: Nutarârsûb sânganut!
42 Mel. 595a.

Rejoice in Jesus' birth. 54.

1. Jêsub inûvia

Kuvianadlarpoк.

Nutakkavut Gûde tagga

Inungorsimajok.

2. Tâpsoma aulapâ Sillaksoar illûnât; Nunaksoarmiut nippâ . Inôjutigivât.

3. Tatta! Engelinit Anginersauvlune Illa Kangusulugane.

4. Atâtab sângane Tuksiupâtigut. Pâmagvigilavut attê Tâmna attanivut!

\section{2 e. Mel. 159.}

Wisdom and power to Christ belong. 64 .

Iksivautaksoarminit

Nunamut akkartor Ajortut nellipsarlugit NertoræksaudlarpoK. Jêsub nutarauningata Naglingninga sakKertipâ. Innornarmêk! illûnainit Nertortautsainarit! 


\section{Mel. 61.}

Kommst du nun Jesu vom Himmel. 40.

1. Kilangmit nunamut akкalerkît Jêsusiga?

Kilaklo nunalo attausênangortuksauvâ??

Jêsusele inungolerkovâtit, Assiojuksaudlarnima?

2. Adamekut assiojilaurtamnik tamainik,

Jêsuse âniarnut tokkungnut kaitsivotit.

Illannâra! asserudlarnerale

Pêjarpat, inûleravit.

\section{Mel. 146.}

Ich freue mich in dir. 41.

1. Kuviagivagit

Nutarar Jêsusiga!

Tikkidlaleravit, Salutipagit illa!

Nippe кuvianaк:

KattangutaulerpoK

Uvamnut, illænâk!

Gûdipta Erninga.

2. Nâlegaviksûb Gûb

Nellonartorsônine

Akkartipâ, arnab

Timânut pitilugo ;

Kotsingnerub Gûdib

Pângat tikkipânga
Nutarârsûlertor

Taijak: Jêsusiga.

\section{Mel. 157.}

Fröhlich soll mein Herze springen. 43.

1. Illa Kuviasukpunga, Engelit serrijut Illagidlarpânga Ahâk! akkimuksidlarpor, Imâgle okokse; Kristus inûlerpok.

2. Uvlome pivor nunamut, Gûdipta Erninga, Sillaksoarmiut Mâne kappiasungningit Pêlerpait; pivlugit Mânna inûlermat.

3. Gûdib nachogigunigit Inuit kattaktut, Inûnajangilar ; Assiokogunittigut, Ajortut pijavut Tigunajangilait.

4. Piniarnivut nuname Ingminut illivait Pitlartaulerlune, Saugak toккоvoк pivluta; Uvagut Gûdemut Nâpkigijauluta.

5. Nergjutit nerchevianne Tamapta imâgle 
Oкautivâtigut:

Allarsigik ajornisse,

Kailuse tamapse,

Kattangutigigapse.

6. Tagva kaititse tamapse, Angijut mikkijut Ômunga satitse! Nagliktok nagligissiuk! Ômamut kaumasek Ullernaigissiuk !

7. Ajornernik ikpigijoк Ômame, ingmigle SænnikteridlartoK; Mannigorle; nagvârame, Jesusib tokkungat Inûlingmagole.

8. Pattangajose tamapse Kiksartut ômamut Ajornipsingnigle, Okpileritse, Jêsuse Tikkimmat; ômunga Nâpkigijaugapse.

9. Piulijigênarlagit! Tamâne nuname Ungagênarlutit, Innôgutiksarsigamga, Tarnipkut nellâgut Nekkorsijauvlunga.

10. Nênertorungnailerpânga Ajortub, timingnut Pigangne pijara. Ivsornaitinga Jesuse!
Mamaitut, tokkungnut Aungnullo pêrangne.

11. Pivlutit ivsornaipunga, Mânnakut illingnut Pigijæksauvunga; Pinnarnarsitigivagit Opinak, nertornak, Pigênarlagille.

12. Udsertutsainartiminga Illingnut, ômamut Nippitsainarlanga, Angergarvigingmilagit Innôsex soraerpat, Pâne nertorlagit.

\section{Mel. 54.}

Wir singen dir Immanuel. 44.

1. Immanuêle Gûdivut Inôgutigidlartavut Omamut nertorpaptigit, Uvaptingnut tikkinavit. Hallêluja!

2. Paradîseme angermat Gûde tillijomavlutit Inungnut kattangnertunnut,

Uttakkivâtit okpertut, Angijomik.

3. Piluarpâtit Dâvidib, Pairsijub inukotingnik, Imna kuviagilauktat 
Illingnut tuksiadlarmat, Pingmat imâk:

4. ,Zionemit Nâlegarut "Tikkiletôk uvaptingnut „Agviartivut pêrlugit ,Jâkokut piulilugit. „Hallêluja! “

5. Mânna tikkisimajotit, Nerchevingme-ileravit Akluvlutit; tamaitale Akluitipattit kilangme Hallêluja!

6. Kilait perkutigilugit Nuname tujormivotit Inungmit amâmaktotit Engelit opigivâtit. Hallêluja!

7. Imarbiub kiglinganik Gûde âkiksoijotit, Ivingne innangavotit. Pivluta inûleravit. Hallêluja!

8. Tamainut kuvianartotit, Siornioralloartotit, Tamainullo nellojunut Mannigoiniksauvotit, Hallêluja!

9. Inungnik raikojijotit Unnuktut ômigivâtit. Ikkitut illalliorpâtit
Inungnik illannârtotit. Hallêluja!

10. Gûd! uvangale kittorngait

Ômamut erdligivagit, Nâmaktomigle pinanga Ajoksartunga piginga, Illûnarma.

\section{4\%. Mel. 132 a.}

Ich steh an deiner Krippe hier. 46.

1. Nerchevingme nanger-

Inôjutigijagit,

Jêsusiga Kaitsilunga

Tunnitsivigivagit,

Uvamnut tunnilauktarnik,

Ômattimnik illûnamnik,

Nâmagikit tâpkoa.

2. Erniangokârtinnanga,

Erniangolaurtotit

Pivlunga, annerivlunga

Jêsuse naglingnernut,

Sulle illitarinæglo

Kittorngarijomavlunga

Issumagilaukparma.

3. Tâktoksoarmêtillunga

Tokrolunga ômane,

Igvit sekrinijâramga

Kaumalerpor illumne,

Kuviasulerlungalo;

Okpernerub kaumasinga

Kuvianartotôngmât. 
4. Omattiga Keagangat Sænnikteridlarlunga, Nipliutivarma igvit: Ajornitit pêrpakka, Kiksarnaglo naglikapkit, Pigama inôkovlutit Pivlutit tokkojunga.

5. Taimaimat nerriukpunga, Angerniarparmalo, Jêsusiga, ômattiga Inniksaulerkovlugo Illingnut, attê kailaurit Ômattimnut, najorlagit, Kuviagilagillo.

6. Erkailertuksauvungale, Kuviagijæksattit Mikkilermatta illumne, Gûd pingortitsijotit, Sanniktitulle-itunga Nachogijomangilarma Ajoralloartunga.

\section{7 ๑. Mel. 7.}

Den die Engel droben. 48.

1. Engelit pânêtut Serritigijangat Jêsus akralaukpor Kivgartoromavlune.

2. Aklugalloartub Akluitipâtigut, Nutakkab sangêtub Pitsartutipâtigut.
3. Tarnîk! imgilaurit

Nertoromavlugo, Asserornartomit

Piulilaungmattit.

4. Attê illûnata

Imgervigilavut

Engelit pânêtut.

Serritigijangat!

\section{Mel. 230.}

Frieđe mit dem höchsten Gute. 49.

Ullapirsauteкarpogut Gûmut, ôma naglingmattigut

Taimak angijomik aglât, Erninga tunidlalermat

Kilangmit tokkomut pivluta. Tagva ullapirsaut illa.

Pêjaijor nuname

Kiksarnartunigle

Illuptingnit;

Taimaimallo

Okautaule

Nunalingne illûnaine!

\section{Mel. $14 \mathrm{~b}$.}

Auf, Seele, auf und säume nicht 50 .

1. Tarnîk tuppalerit, ahâk!

Kaumajok nuivor,

Uvloritsab okautigivâ Nâlegar tikkitor. 
28 Jêsusib Kristusib erniangoninganik

2. Kaumajok tâmua kammagiuk,

Nuijor pivlutit!

Pitingmattit nutakkamut Jêsumik taijamut.

3. Mâne tarnît ajoksartok NekkoKsitiksamik Ajunginerpângojomik Nellipsaijungnarpoк.

4. Gûb okausingit nâlekit Tâpkoa aulangitut, Tessiormattit tâktomit Kaumajoksoarmut.

\section{Mel. 310.}

Morgenstern auf finstre Nacht. 51.

1. Sekkerngub nuilertub Akkisuktipâtigut; Jêsuse! Kait, attê, Kaumalinga illumne.

2. Kaumajoksôjotille! Tarnimnut itterille; Kait, attê, Jêsuse! Kaumaksalaungmingale.

\section{Mel. 95.}

Herzensknabe :,: 54.

1. Nagliktiga Jêsusiga!

Nunab pingit illûnatik Illiktun-itsengilat, Manigoijôtogavit
Illa sænikterijunik, Mannigolaungmingalo!

2. Betlehem ivlernartotit, Illa Kuvianarpotit; Illingnit pingmat tâmna, Idluartitsijomajok Uvaptingnik ajortogut; Tokrotailitivullo.

3. Nutarak tattamnartotit Ikkajunga, næglingnernut Ônarsilerkovlunga, Nunamelo ômattimnut Ungagitsainarlutillo Tailutit, perkovlunga.

\section{1 at Mel. 95.}

O du holder süsser Knabe.

Nutarârsuk erdlingnartor, Nangminikka illûnaita Illingnut âlaulakka! Kungalaunga! kailerpunga Senningnut sêrkortorlunga,

Pilauruk ômatiga!

\section{Mel. 10.}

Ach Christnacht und sein Leiden. 57.

Kristum inûlervinga,

Ânialervingalo

Kuviagijomavavut

Nâksaungitoksoarmut. 
53. Mel. 10.

Die wahre Gnadensonne. 59.

1. SekKerngub miksezârtub Nuilerpâtigulle;

Kaumarkojomavluta Ajorungnaitilutalo.

2. Jêsusib nutarârsub Imâgle pivâtigut: „Kiksadlarniarase „Mannigoromagapse.“

3. Sorruserle Jêsuse! Inûleravit mâne. Nunab pinginit tamainit Ungagijomavagit.

4. Kanok illaliorlagît?

Kippiniarigapkit; Nunamut tikkinavit Innuit saimarlugit.

5. Pingortitsigaville Tamainik, Kanor mâne Akludlarkît, tamainik Aulatsijotôjotit?

6. Kanoxtôk nutaraunit, Nunamullo tikkinît Âkiksûtigilâkka Inûlijaukovlunga.

7. Illingnêtsomavunga, Nachoginiarnanga, Tuksiarvigigapkit Ubvaruk ômattiga.
8. Uviningme sakkernît, Sujungaitigilav ut Ajortoliksôgapta Sângne, illa tamapta.

9. Inûlernît Jêsuse, Aniallo nuname. Kuviagijomârpavut Kilangme najorlutit.

10. Pâne soraingitomik Nertoromavaptigit, Sângnut pâmakuptale Keanangitovingme.

\section{Mel. 58.}

Glücksel'ger ist uns doch keine Nacht. 61.

1. Kuvianarnerpâk unnuak, Kaitsilauktok nutarârsungmik,

Tattamnadlartomik, Pingortitsijomik, Akluvlunele illijaujomik Nerchevingmut.

2. Gûd, Gûdivut nellekangitok!

Naglingningalo nâpkiniktok,

Kippipor imungnik Piulijom mut,

Nelliunek uttaknivâ Jârit Viertausendit. 
3. Nelliutingmat, Atâtamit Annijok ânanauvingminit, Atâtab pingmago;

Taimak inuit

InôKovlugit toKKotigivait Piulivlugit.

4. Uvinik auglo tâpkoatuak Nuname nangminerilaurpak;

Perkutekangilak,

Sunamiglônêt

Inniksamik sinnigvingmiglônêt

Peкangilak.

5. Tautua tækkominalaungimat

Taimak pivlugo nachogivât Mittautigivâllo

Okperinago;

Kattangutingillo kangusukput.

Tâpsominga.

6. Nellekangitor ânianga Torkomut kappiasungninga

Tuksiarnermulle,

Auktun-itomik

Kidsijârlune, kuttit timânit

Korlolerput.

7. Kellaklugillo avatingit

Puttorpait aggangit ittiggangit;
Kikkiaktormatsuk Senningajolingmut, Tagva Jêsub piulivâtigut Kattangnermit.

8. Ômattivit illagivâtigut Tikkinît kuviagiguptigo Serrigilugolo ; Taimaikovluta, Pêjariakarkârpattit tagva, Ajornivut.

\section{4 at. Mel. 215.}

I denne glade Juletid.

1. Kuvianartoliksoak

Nelliutilermingmat,

Sappingitaptingnut tagva Gûd nakoridlarlavut! Erninga mânna tikkitor Nerchevingmut illimajors Salutidlarlavuttôk!

Nuna aglât sajulerle Silaksoarme illûnâne Nertortaudlarmat Jêsus.

2. Dâvidib erniarsunga Sullelo Gûdiojox

Kilangmit akKalaungilâk Ajornivut pivlugit? Aukang-illa, sapperame Inuit asserornerme Takkovlugit кailauкрок. Aniajomadlarnermut Simmerluta, takkovarut Gûd rattangutiptitut. 
3. Kujagidlarlavut tâmna Unêt sangêdlarluta, Hôsiana, Hallêluja Attorlugik pilaukta! Tangmầrviptingnêdlalermat

Inuit piulijingat.

Sâtanit illûnatik Sajulerlit, tussaramik Sâlakartut nippinginik Uvlorsiorviptingne.

4. Gûdib ningarungnaerninga Kaujijaudlarpor mânna, Ernine tillilaungmago Piulijiksaptingnut. Illûnâne kaujijaule Jêsusib tillijauninga Siorniorviptingnut. Tagva kuviagilavut, Siarklernek illivlugo Jêsupta ômatânut.

5. Sorlo unnuak târnerpâk Sekkinermut pêrtaungmat, Kiksarnera pêjartauvok Issumarsarsiormut Gûmut nagligijaunimnik . IssoKangitoksoarmit, Kattangutigingmanga. Engelillo oKausingit: „Ullapirsaumik nuname!“ Tussarôtsainarpakka.

6. Hallêluja, sorsungnivut Soraerpok sâlakarmut. Kinalo kiksarajarkâ,
Serrerkojaudlarapta? Gûb inungit, niplilaukta: „Hallêluja, pigivara "Gûb inûblo erninga! "Nâmaksivor tussunera, "Tessiortekalerama "Kuvianartovingmut.“

\section{Mel. 39.}

Gott unserm Gott, welcher im menschlichen Orden. 64.

Gûd, Gûdivut, Kattangutigijavullo

Nuname inûlermat âniangmallo,

Pivlutalo tokrungmet, mânna tamapta

Nakoridlalavut kujagivlugolo.

\section{Mel. 240.}

1. Jêsub inûlervia

Nelliumat mânna

Kuviasulerta

Kujagivlugolo,

Nakoridlarlugo !

Pingortitivulle

Nergjutit iglungænnut

Illivât aklujotut;

Illâle taimaimat

Kujagivavut,

Piulingmattigut.

2. Engelib tautua Attoromangilâ, 
Miklilerlunele

Kivgatun-iname

Nuname-idlune,

Nagligigamigit

Inuit, aungminut,

Pissiarivlugit,

Aungmik kiægungmat,

Opigilavut

Nakorilugo.

\section{5\%. Mel. 22.b.}

1. Nutarar nakorivagit

Nuname inûleravit,

Gûdiogalloartotit

IssokangitoKsoarmit.

2. Adsigilerluta aglât

Pigavit nâgligosumut, Ikpigikattalaurpotit Kângmiglo merngortornermik.

3. Tarnikkut âniagavit, Aungmik kiagulaukpotit, Illingnik tunidlaravit Pivluta tokrotaugavit.

4. Pivluta tokkolauravit Akkilêlutale sumîk? Timivullo ômattivut Tunnidlarpavut illingnut.

5. Tâpkoa innigilikit Missuktilugillo aungnut, Timingnut kattaktitomut Adsiotilaungmittigut.
6. Tamainik pingortitsijoK Gûdeojok inûlerpok, Tagva rujagidlarlavut Taimak nagligingmattigut.

7. Ômamut nakorilavut Illa piulingmattigut Inûlerninga tokкunga, Erkarlavuk sorairata.

\section{Mel. 14 b.}

1. Gûde nakoridlarlavut Mânna inûlermat, Uvaptitun-itsomangmat, Ajortokangitor.

2. Tâpsoma pingortitipta, Tammalauraptale, Piulijartorpâtigut Idluiniptingnit.

3. Taimaimalle opigomut Erkaromavavut, Ungagijomavavullo Nunamêtilluta.

\section{Mel. 83.}

O verehrungswürd'ge Nacht *Anh. 14.

1. Unnuak ussornartok! Kaumajôvlutit inungne, Jêsuse naglingniktok Erniangongmat nuname, 
Piulijartorluta

Tokkominut tamapta.

2. Gûb sillaksoarmiut Taimak naglikpait ernine 'Tunnivâ tâpkonunga

Piulijaukovlutale; Tâpsomunga okpertut Assioniængilat.

3. Kuvianamêk illa Jêsusib inûlervia!

Kaitsingmat tarniptingnut Piulijaujutiksamik, Kaujitidlarlutalo Kilak angmarmat mânna.

\section{Mel. 155.}

Süsses Evangelium. *Anh. 5 .

1. Okautsit tussanertut Mannigornadlartut illa Inungnulle

Piulijaujomajunnut

Tamainut:

Gûd uviningme sakkermat, UllapkuteKartiluta, Tunnergutaunerminut.

2. Ullapirsauteкаrрок Mânna nuname tamâne, Mânna Gûdib

Kûviagivâtigut, Aungminut

Jêsub âkiksormattigut, Tussudlarmallo tamapta Piulijaukovluta.

\section{Mel. 230.}

0 wie beugt uns das zur Erde. *Anh. 9.

Opigosukpogut illa: Nagdligosumut inûlermat Pivlutalo tokkolune; Gûb uviningme sakkertub Aungminut piulivâtigut, Kejungme nâmaksitipâ. Hailig Nâlegar Gûd, Pitsartudlartor Gûd! Inônivît Tokkonivît Ajortunit Piulidlalaukpâtigut.

\section{Mel. 185.}

Süsse Wahrheit, die mir's Herz genommen. *Anh. 11.

1. Miksekârnerub tussarnidlartub

Kiblikpâ ômattiga:

Gûde pivlunga inûlilaungmat,

Tokromut ailunelo. Illuga ônarsititaulerpok Inûlijaudlarama nutâmik, Anernera tarnigalo Opigosuktitauvuk.

2. Avatikka Kuviasugitse, Gûdeme ômajome:

Pivlungataur uviniolaungmat

Piulijomavlunga. 
erniangoninganik

Jêsub attinga tussarangapko,

Omattiga serridlarpok tagva,

Piulijigigapko

Nertoromadlarpara.

\section{Mel. 168.}

Wiederholt's mit frohen Tönen.

* Anh. 13.

Tussarnertunut nippinut

Tuksiatsainalerta:

Nergjutit nercheviænne Gûde innangalaungmat. Opinadlartor tamanna Erkaumajautsiarkovlugo, Niplertoratsainarle: „Ahâk nutarak una!“๘

\section{Mel. 230.}

1. Attetôk Gûde tamapta Imgervigidlarlugo pita Kujagilugo ômamut! Pattanganivut tâpsoma Omamiutarilaungmago, Erninelo tillingmago, Takpângat kilangmit Tamaunga nunamut; Saimanermut

Pingmat illa

Tagva mânna

Nakorilavut attinga.

2. Illa Jêsus Gûb Erninga
Erniangogavit pivluta Perkutigidlarpaptigut, Pigidlarpaptigillotauk Tokкungnut rellulauraptigut

Kannoêtunit tamainit;

Perkutaukovluta

Sorairata mânna

Illingnulle;

Ikkajorit uvaptingnik Tunnukonnata illingnik.

\section{Mel. 168.}

1. Nelliutok serrinartor Sakrersimavok mânna, Pingortitipta nellâgut Illagilermattigut; Mânna nellautsimarikput Angerningit atâtanut; Tikkituksak tikkipoK Ajugaujomik taijar.

2. Ullapirsaumik kaitsivok Tâmna ômattiptingnut; Sujuktaujullo Adamut. Omunga âkiktauvut, Inôser piulijaunek Piniarutigidlarpak Pivluta, Gûddivullo Ullapirsarpâ illa.

\section{Mel. 167.}

Uvangatauk ômattimne Attaruililerlanga, 
Nergjutit nercheviænne Ungagitsainarlago; Jêsusigalo nellâgut Inûlermat pivlunga, Nâleklugo opigomut Illupkut nertorlago.

\section{6\%. Mel. 184a.}

Piulivlunga tikkinavit, Âniarnullo tok̊kungnut, Illûnamnik tunnilerpagit; Kippilerlunga tarnipkut, Nutarârsuk, kuviagigapkit Sakkijârvigilaungminga, Anernipkullo erkilagit Gûdiga, Kattangutiga.

\section{Mel. 218.}

Tarnîk serrilerit Gûdipta Ikkajoriartormattit Nellungilaille illûnaita Kappiasûtigijattit, Kiksarungnaikojomavâtit Innertuitaililutille; Kuviasugit, pivâtit, Tikkidlarmallo naglingniktor
Saimarsaijorlo ikpigivat, Illale pilorikpotit.

2. Gûdivut tættigiluaruk Illiktut uvineKarmat. Omamine illungertorpoк Piulijomadlarlutit. Innangavia tækkosaruk, Tâmna inûlermat pivlutit; Imgerit: Hallêlujamik! Issumangit mannigornartut,

Kujalijutigællugille, Pingmattigut, Kuviasukta!

\section{Mel. 11.}

1. Pinnarnartok nutarak Tættigigapkit mânna, Illikullo pivunga Kilaub pinginik illa.

2. Naglingniktor Jêsuse, Tarnimalo timima Maunga tikkinaville Nertoromadlarpâtit.

3. Gûdivut inûlernît Aut tokkullo ikkitit Kuviasûtigivavut Perkârata kilangmut. 


\section{JÊSUSIB KRISTUSIB KIPPIJAUNINGANIK JÂRIBLO NUTÂB PIGIARNINGANE IMGERUTINIK.}

\section{Mel. 165.}

Heut lässt er uns zu gute. $* 96$.

1. Uvlome sivorlerpâmik Jêsus âniagame

Kovivait kuttiarsuit Auk, kippijaulerame; Âniaviksaptingnit Nâksaungitorsoarmit Ajortunillo tamainit Piulivluta aungminut.

2. Tagva erkaumadlarlavut Idluitullinivut

Kakkialerutigilavut Kivgartorungnailugit; Jêsuse kippijaungmat, Timinga âniangmat, Ômattivut ajortuggit Illa tagva kippijaulit.

\section{Mel. 166.}

1. Erkarsiuk piluarlugo, Uvlome aune ôma Sivorlerpâk kovingmago Piulijomavluta, Âniatigællutalo, Assiojuksainaugapta Kutsaservigidlarlavut Kuviasuklutalo.
2. Piulijiga piginga, Jêsumik attekaravit, Ikkingnut inûligamga, Idluarsitigamga, Pingortinamga aggangnut, Aungnut piuligamga, Taimak pivlugo kissivit Pigimarilerparma.

3. Uvlut tamaita âniat Toкkullo ikkitillo, Erkomatillungalônêt Sinningnialeruma Ómatimne innexarlit, Kuviasukovlunga; Tagva nakorênarp git Mattomanêtillunga.

4. Axtoleruma timimnik Mannigorlaungmingale, Âniarnullo tokungnut Piulidlalauramga; Toккolerpallo timiga Ivsume sujuklune, Tarniga uttertilugo Ômartitsomârparma.

\section{Mèl. 58.}

Heil'ger Name Jesu. 70.

1. Ajungitorsoak Jêsub attinga! 
Jâriblo nutâb pigiarningane imgerutinik. 37

Kiale nakorivâtit illa Nâmaktomik?

2. Pitsartudlartorlo

Ĵ̂sub attinga!

Pilorikpunga piuligamga

Ajortomit.

3. Erdlingnadlartorlo

Jêsub attinga!

Sapputsaujut piulijaujullo

Ungagivâtit.

4. Arlingnartorsoak

Jêsub attinga!

Okpertut illingnik piksakarput.

Sorairatik.

\section{\%อ. Mel. 146.}

Nâlegavut mânna Kujagijomavavat, Jâreme rângertome Sapputilaungmattigut. KingorngagullotauK Saimartilittigut, Pairitsainarlugit Tamaita okpertut.

\section{Mel. 2za.}

Das neugeb orne Kindelein. *95.

1. Nutarak Jêsusearsuk, Naglingnartoк, Kaitsilerpor,
Jâremik nutâmik ama

Inûtitaminut illa.

2. Tamanna Engelit aglât Kuviasutigilerpât, Imgidlarpullo sillame, UllapкosertaungmatGude.

3. Ullapkosertaukpet Gûde, Illænâriguptigolo, Kina akкonaijungnarka Uvaptingnik mâne tagva?

\section{Mel. 151.}

Helft mir Gottes Güte preisen *99.

1. Gûd nâlegaviksôngmat Nertudlarlavulle, Ômamullo kissiat Kujagidlarlavut; Piluarlugo mânna Nutaungitor nângmat Jâre nutâk pingmallo Kuviasulerta!

2. Gûd Atâtak! pijattit Jêsus Kristusekut Uvaptingnut, illûnatik Nertoræksauvulle; Kennuvigivaptigit Jâreme nutâme Serngnigiluarluta, Piksakartitigut. 


\section{Mel. 165.}

Jâr nutaungitoK Kângermat, Kuviasudlarluta

Nutâk pigialerlavut, Issumatârlutalo ;

Kennuvigilavullo

Jêsus Piulijivut,

Nutângortitserkovlugo

Uvaptingnik illûnâne.

\section{9\%. Mel. 10.}

Nun lasst uns geh'n und treten. 67 .

1. Nâlekamut ailerta,

Tuksiarvigilugo,

Pairijaujomavluta

Mattomanêtilluta:

2. Sorlo arnab nuname

Pairingmagit kittorngane

Nangiarnartolingıut

Perkonnagit nellînut.

3. Taimaktauk Nâlegapta

Kittorngane tamaita,

Nangiarnartokarpat

Serngnigivaille illa.

4. Taimaktaur nellungilavut

Pinniarnerlukapta,

Sullijavut Jêsusib

Pitsartutingipagit.

5. Jêsuse nagliktivut

Tamaita ômattivut
Aungnut âkiklugille

Tamât innôsiptingne!

\section{Mel. 70.}

O Haupt am Leibe. 68.

1. Illagêktunut niakotôjotit Gûd, illûnata pitsartutittigut!

Idluarijarnut piluta

Pairsinerniglo opigukluta.

2. Jâreme mânna nungulermijome

Erkailerupta, nâpkigosungnernik

Illûnaptingnut, ajortogut; Naglingnernut aumatipaptigut.

3. তvlome nutâmut Jâremut pivogut,

Tuksiarluta: tessiormittigut,

Illagêne illunarsoata

Naglingnernullo pairijauluta.

4. NâlegaK Gûde, nagliktotôgavit

Jâreme nutâme kuviagijarnut

Inôniarkovluta sângne, Nâpkigiluta pairittigulle. 
Jâriblo nutâb pigiarningane imgerutinik. 39

5. Igvit tamapta kittorngarittigut

Illagêkluta, aulingnut torKungnut,

Okpernermut ungagilutit, Illûnatalo nâlelerlutit.

6. Inôgutivut, igvit pitôgavit Illûnaptingnut, Jêsus nertortaugit.

Tamât arvertarvigittigut Nutâmik inôluta illingnut.

\section{Mel. 10.}

Wenn wir uns kindlich freuen. 360 .

1. Tamainele Jârine

Naglimut nutakkatut

Kuviagiguptigo

Piulijipta aunga;

2. Âniangalo pivluta Angerarkârtinnata; Okpermarikuptalo Tækkojomâraptigo ;

3. Orautsillo imaitut: "Tâpsomanêlerapta, "Angergaromârupta" Mannigorvigivlugit:

4. Mâne kiksarviksavut Puigorniarpavut; Jêsub najormattigut Nekkoksivut illuvut.

\section{Mel. 22.}

1. Nagliktavut Nâlegavut Omamut tuksiarpavut, Najuênaromavlugo, Nuname inôtilluta.

2. Tâmna illa illuptingne Missigitsainaruptigo Nellâgut kuviasumut, Tagva sullitsainarpogut.

\section{Mel. 9.}

Wenn dein Herze mit uns ist. 71 .

1. Jêsuse ômattivit Illagikpattigut, Saimarsainîlle tamât Tukkisidlarpavut.

2. Kaujimajotôgavit Erkarsautiptingnik, Pigittigut nutâmik Illa illûnata.

3. Unêt idluitoguit, Ajudlaraptale, Pigiluta tamapta Nâmagênartigut.

4. Tarnivîllo tamaine Illagilittigut Ikkitillo, Gûdivut Nakoridlarpavut. 
5. Akrunaptingnêkuvit

Unêt tækkuksaunak,

Tarnipta missigivâtit

Nakoridlarlutit.
6. Taimak illagivlutit

Kuviasukpogut,

Tunnitsivigigaptigut

Ullapirsautingnik.

\section{KRISTUSIB ÂNIANINGANIK TOKKUNGANIGLO ILLUVERTAUNINGANIGLO.}

\section{Mel. 36 .}

Gott fällt in einem Garten hin zur Erden. 73.

Gûde perorsêvingme pâmangavoK,

Tokкomullo kappiasudlalerpok;

Pingortitsijok pingortitaminut

Nekkorsijauvok.

\section{Mel. 54 .}

Geh mit ihm, o Gemeine! geh. 74 .

Illagêt! illagisiuk,

Taikunga perorsêvingmut;

Tækkolugo kidsijârninga,

Aggut aglât kussilertut

Kềnanganit.

\section{Mel. 99.}

O Anblick, der mir's Herze bricht. 75 .

Jêsusiga, tautukapkit

Perorsêvingme ânianitit,
Ômattipkut kibliktauvunga,

Tokкomut aglât tarnikut Siornioravit pivlunga! Kanoktôk mânna pigilaunga!

85. Mel. 146.

Bedenk' ich Jesu Buss. 77.

1. Kidsijârtor aungmik

Timânit kussertomik,

Kappiaktoamut

Jêsuse tautukapko,

Illungertorlune,

Tokkomut kiksartok

Pâmangadlarlune

Sajuktor kêajor;

2. Tagva ômattiga

Sittijor aumalerpor.

Jêsub ânianga

Ullernaigidlarapko,

Gûdib ningausinga

Tagva tautukpara,

Taimaktauk âniangat

Piulilaungmanga. 
3. Kujagidlarpara,

Kibliktaudlalerpunga

Tautukapko illa

Keadlartor pivlunga;

Ôrksub Kakkangane

Allaromaerpara,

Inôtillungale

Ungagênarpara.

86. Mel. 184 b.

Jêsusib ânianingita

Erkaumajauvingat mânna Nelliutingmat, âniajok Tokkotaujorlo pivluta Aiparitsainaromavara, Okausingillo kingorlit Ômamiutarijomavakka, Puigorungnailugillo.

\section{8\%. Mel. 185.}

O Betania. 499.

1. Betania ullapirsautelik Annerijaudlartotit!

Illannâma ittervigilutit Illingnêkattalaungmat, Kugvinnut kippivakpunga sulle

Marîatut saimartaujomamut!

Najorninganêkuma

Betaniamêkôrpunga.

2. Betaniamiut anernipkut Nellipsaromavakka,
Jêsub ittiggangita sânginut

Ingitsartolerlunga.

Ajokertûsingit tussarnertut

Nâlegomavakka Marîatut, Ajunginerpâk tâmna Annerijomagapko.

\section{Mel. 10.}

1. Ahâk ! tamânêpunga Illingnut kemnulunga: Piulijiga aungnut Tarniga nekkoksiuk.

2. Perorsêvingmêdlutit Tagva ânianitit Erkarapkit pivlunga, Aumavok ômattiga.

3. Tagvale erkaivunga, Idluitoksônima Nênermattit nunamut, Pivlunga kuglugiatut.

4. Tuksiadlaleravit

Kênarnit aggut kuttit Korlolerput nunamut, Missuktipâllo nuna.

5. Taimak tautukôrapkit

Pinnarênalerpagit; Ijîkka kugviorpuk Sorairatik pivlutit: 
6. Sumigle akkiksarnik

Kaitsijuksaugalloarpîk?

Âniagavit pivlunga

Pigiuk ômattiga.

\section{Mel. 36.}

Ach hochverdienter Seelenfreund. 76.

1. Jêsusiga, naglingnerpângojotit,

Gûdib ningausingat tupvigivâtit;

Gûde ningaksarmatsuk ajornima,

Pivâtit illa.

2. Taimaimat avatitit ivsornaitut

Sajukput, tarnikullo kiksarlutit,

Simmerlunga pivat pijæksaunera

Nagligigamga.

3. Tagva pilaurittôк кujalinermik,

Naglingniktotit illa tarniptingnik!

Pigittigut, ikkingnut torKorluta,

Sâlakarkovluta.

\section{Mel. 14.}

Der Hailand war so todt betrübt. 78 .

1. Jêsusib naglingmattigut

Pivluta Keavok,
Kiksadlartorlo toкnomut, Aungmik kiægukpor.

2. Tautualo tækkominar Tarningat ânia; Aulaumajartitauvlune, Sêrkortolaungmalle.

3. Perorsêvingme keanivit Kugviortipânga Nâlegara najorlagit Pigavit pivlunga.

4. Kanoktôk inưlinartor Kiægungnex tâmna, Aungmiglo akkusimajox, Ômattimnut pile!

\section{Mel. 244.}

Ich blicke nach der Höhe. 79 .

1. Takpaunga ârlorpunga, Illungertorvingnut, Illa kiægungnerit Auggit korlolerput. Tarnimnullo mânna Sakkijâlaungminga, Kappiasungnernigle Piugorkonnanga.

2. Jêsuse errilagit, Illungertortillutit, Aiparilaungmilagit Orksub Kakkangane Aungmik kugvioravit 


\section{illuvertauninganiglo.}

Tautukôlerapkit, Âniarne tamaine Najutsainarlagit.

\section{Mel. 152.}

Christus, der uns selig macht. 80 .

1. Kristus Piulijivut

Ajortokangitok,

Unnuame tiguvât

Sorlo inuartok,

Inuit sullinatik,

Tâmna passidlarpât

Mittautikumavlugo

Kikkiaktortaukovât.

2. Akkerartortotulle

Attannersoarmut

Uvlâkut pitilerpât

Nellojub sânganut;

Passijæksaungininga

Ôma tukkisivâ;

Taimaimat âtaukovâ

Herodesemulle.

3. Jêsus aklunâtsanut

Issakadlalerpât,

Orpinnut kênariktunut

Niakôsidlarpât;

Mittautaunartomiglo

Annorârtilerpât;

Senningajolingmiglo

Erksuktilerivât.

4. Annoraijarlugolo

Nivingatilerpât,
Tagva aune kovivâ

Tuksiadlarporlo.

Sennerkotut, amalo

Nivingakattingat

Allêkutigilaurpât,

Serkinerle tâkрок.

5. Nipliangmat: Gûdiga

Sôgle Kemmakpingâ?

Sêrnartomik imipât,

Niakone nairpâ.

Tokкolermallo, nuna

Sajukpiludlarpoк,

Upkorsoar allikpor,

Kairtut kopludlarput.

6. Uvlor ketterarêrmat

Jêsub senneranga,

Kallugiaksoarmut

Kappijauvok, tagva Auglo imerlo kôkpuk

Ôma sennerânit;

Illale auk immerlo

Uvagut pivluta.

7. Seккiner nippilermat,

Unnulertillugo,

Kejungmit akKartipât

Jêsusib timinga.

Illuvermullo tagva

Illijartortauvok ;

Uvlunik pingasunik

Udsertortaukovât.

8. Kristuse Gûb Erninga Tokrungnut aungnullo Ômættivut tamaita 
44 Kristusib ânianinganik torkunganiglo

Kiblitsainalaukit; Tokkogavit pivluta ErkaitittiguttôK, Kujalidlarkovluta Ômamut illingnik.

\section{Mel. 14.}

1. Piulijipta næglingnartub Âniadlarningit

Pivluta ikpigijangit Kemergolerlavut.

2. Innônermine nuname Kappiasudlartok, Tikkimat nelli uninga, Tokromut kiksarpok.

3. Ajornipta nênermatsuk Aungmik kiægukpok, Tokкublo kaglilermago Tuksiadlalerрок.

4. Engelib tagva tikkipâ Nekkorsitilugo. Nellekangitor âniak Erkarniarlavut.

5. Kênanga ketserarlugo Tækkominaitipât, Kolênga issakautinut Tâp koa killidlarpât.

6. Ajornerit allarlavut, Nivingatimmatsuk, Jêsuse Piulijivut Senningajolingnut.
7. Jêsuse Piulijivut!

Tuksiarpaptigit,

Tarnivullo timivullo Aungnut ubvalaukit.

8. Illa uvlâmit unnungmut Aunârtut ikkitit Missigênaromavlugit Tuksiarpaptigit.

\section{Mel. 216.}

Ein Lämmlein geht und trägt die Schuld. 81.

1. Saugârsuk nangmalerame Sillarssûb inungiti Ajorninginik, tarnekut Siorniorutigivait;

Nukingêrutijauvlune Pivluta tokkotaugame Inôser tunnivlugo; Mittautigijaudlartorlo Kik kiaktormatsuk okarpoK: "Âniajomavunga.“

2. Saugârsuk Gûdeviojor Tokrublo innôtsiblo Nâlegarivâk, Atâtab Ernerivâ, tâpsoma Tunivâ torkomut pivluta. Naglingnek sôngudlarpotit!

Tattamnartitsidlarlutit Tâmna pitilerangne 
Tokкomut illuvermullo,

Kairtut koplortigijanget.

3. Gûd! naglingnivit tokкоmut

Siorniortipâtit,

Kikkitsenut avatikut

Ikkilertautipâtit.

Kappijautimmattit, aggut

Kôktor takkarnit nunamut,

Piulijaukovlunga.

Jêsusiga KanoK igvit

Tagva akkiniarlagit?

Illûnarma piginga

4. Inôtillunga Gûdiga

Allarniængilagit,

Erkitsomadlarpagille

Erkidlaramga igvit;

Ômallo tiglertillugo,

Kaumartigilerapkillo,

Ômamut nertorpagit,

Igvit piulilauramga;

Nâksaungitomut illingnut

Perkutautsainarlanga!

5. Naglingnit uvlut unnuet Imgerutigivara,

Tunnivunga tamainigle

Illingnut, illûnarma;

Ómattima opigomut

Kujalimut nakorimut

Nertutsainadlarlitit!

Ajungivigênaramga

Omattimnut nênerpara,

Puigorungnairlagit.
6. Omattîk angmalerille,

Piksarsiorkongmattit

Piluartomik, kilangmit

Immarbingmit, nunamit;

Nunab pingit pêjartaulit

Allianarningittauk pêrlit!

Ajunginerôjomik

Nagvấrama: Jêsusiga

Aut kovimmajok ikkingnit

Perkutigidlarapko.

\section{Mel. $184 \mathrm{~b}$.}

1. Aiparilaulagit Gûdiga, Âniavingnut tamainut, Jêsusiga sakrerviginga, Siorniudlartillutit;

Tautukôrlagillo pivlunga

Pâmgortotun-ilerdlutit Aungmik kiaptikartillutit Piulijaujutiksamnut.

(Ich stehe mit bewegtem Herzen. * Anh. 37.

2. Ômattipkut aumadlarlunga

Nangerpunga Golgatame, Najulaunga ânianernut Mannigortaujutiksamnut. Kovisinernît aungnik illa, Tokrolernîllo pivlunga, Pitjutauvuk Gûdemut mânna

Ullapkotiksakarama. 


\section{Mel. 36.}

O Lamm! das keine Sünde je beflecket. 82 .

1. Saugârsuk Jêsuse! ivsornaitotit,

Ajortunut sujuktaungitotillo,

Sulle kappiaktoajuksauvotit

Aunâdlarlutit.

2. Opaktauvotit pijaudlarlutillo

Mittautigijauvotit angijomik,

Tigluktauvotit erksârtaulutillo

Ânertauvotit.

3. Illa aglât ôminartitauvotit Kejungmullo kikkiaktortauvlutit,

Kemaktaujuksaulaukpotit Gûdemut,

Tokkolerlutit.

4. Saugârsuk! pilauruk Kujalinera

Naglingnît sakkertilauktat pivlugo,

Ôminaut uvamnut pijuksaulauktor

Nangmalaurangne.

5. Inôsera illûnaralo illa, Perkutaule illingnut sorairane,
Pivlunga ikkekartitaulauravit

Tоккоgavillo.

\section{9\%. Mel. 166.}

Pilatusib Nâlegara Aularkojomavlugo, Pitlaræksausugingilâ Issakajaukovâle. Gûb inûtitangitale Kikkiaktortaukovât, Pêruk, pêruk inuk una, Nipliutigidlarpât.

\section{Mel. 218.}

Dir haben Arbeit meine Sünden. *Anh. 218.

1. Idluinima nênerlutit Ajornima pitlarpâtit, Kia illiktut ajortuggit Nagliklugit piulivagît? Gûb ningautâ attoræksara Pêrpat, tokkogavit pivlunga

Kiksarungnaitidlarparm a, Kannimasikka pigivattit Pivlugit âniadlaravit Tokkomut aglât, Jêsuse.

2 Ajortut pitlartaujutitit Illingne merngoêrserput; Kannimajut akiktauvlutik, Ikkingnit ajorungnaiput. 
Pivlunga toккub attortavit,

Aukpit, pêjaijub ajornimnik

Mânna piloriktipânga, Attorlago ullapirsautit, Korlorvigilingalo aukpit, Piuliklernit pigilago.

\section{Mel. 165.}

Fliesst ihr Augen, fliesst von

Thränen. 83.

1. Kugvileritik ijîkka Ajornikka pivlugit, Aumaniarit ônattîk, Erkaruk piulijît; Ôma pitlautiksara Ingminut pitinerpâ ; Illa âniajutingit Mânna ômamiutikit.

2. Jêsus ibGûb saugârsungat Ajortut agganginut Pititaujub simmerluta Pitlautiksavut pêrpait; Adamemit pijavut Kattangmat ajortomut; Ajornivullo tamaita Âniajutigivait ôma.

3. Jêsusimale kollêne Issakausijartunut Kununane mannimivâ, Kina kibliktaungilâK? Ômame aumanermut Tautuklugo Gûdivut
Sorlo ajortullijotut

Taimak issakadlarmatsuk.

4. Taimak ajortullinimnut

Pitlartaujuksauvunga, Tâpsomale naglingnermut Âniatigivânga ;

Tarnîk nautsertoleruk Âniâ kiblingnartok, Pitlaræksaungitub ôma Pijaukongimattit illa,

5. Âniavinginut tamainut Tarnîkle aipariuk, Tamât siorniorninga Omamut erkariuk, Niakôsertauninga, Mittautigijauninga; Tamaita ikkingit auggit Inôvituarilikit.

\section{Mel. 54.}

In dieser peinlichen Gestalt. Aumavunga tækkogapko Jêsus âniadlartillugo Sakkertimmatsuk inungnut,

OKautauvok: tâmna inuk Tækkosiuk!

\section{Mel. 168.}

Wiederholt's mit süssen Tönen. 84 .

1. Tussarnertunut nippinut

Tuksiaritse, imâk,

Tailugolo sorairase: 
Ahâgle inuk una! Sumigle pilauktok una? Ullernaigituarapko, Âniak tautuklugo Soraerniangilanga.

2. Pitlarapko, erkaivunga: Ahâgle innuk una! Nâpkiniktuble tautungat Mannigudlalerpânga. Kênangat kidsijârningat Aukejârsimaningallo Mitsuktipak ijikka, Angergarkârtinnanga.

3. Inûb ôma niakoa Kollênga ikkilertak Illutorsimajuk ijik; Ahâgle inuk una; Ânertaujok illûnane, Ungagijæksaringilagît? Aukak, erdligilagit Takkonâtsainarlutit.

\section{Mel. 36.}

Herzliebster Jesu, was hast Du verbrochen. 86.

1. Nagliktok Jêsuse! sumik pilaukît,

Taimak pitlaræksangortilaungmattit?

Illa sôg tokKotæksangortilutit.

Passilaukpâtît?
2. Idluitullijotut annauvâtit, Mittautigimut kikkiaktorpâtit,

Sêrnartorlo akkullugo sungarmik

Immertipâtit.

3. Sôgle angijomik pitlalaukpâtît?

Illa ajornima pijautipâtit, Taimak âniajuksaudlalaukpunga,

Illa uvanga.

4. Tagra illa tattamminiarpunga,

Igvit ânialauravit pivlunga;

Illa ajorniâlukka illingnut Okumailaukput.

5. Ânianitit kikkiaktortaunît Tokkullo ômamiutarilak$\mathrm{ka!}$

Kivgaujungnaitinnamga Sâtanemut Kujagivagit.

\section{Mel. 185.}

1. Jêsusiga! tautukôrangapkit,

Kikkiaktortauvingnut Sajulerlutit majoraravit, Kakкamut Golgatamut, Piulijomavlunga uvanga, Âniarnut aungnut toKKungnullo, 


\section{illuvertauninganiglo.}

Ômame kiblikparma

Taimak nagligigamga.

2. Âniavilllo akkiksanganut Timimniglo tarnimnik Tunnijivunga kibliktaunermut, Aungnulle mitsuktikik; Onarsitiuglo ômattiga, Tokkogavit Kejungme pivlunga,

Puigorungnaitinga! Âniatigigamga.

\section{Mel. 79.}

Wie preis' ich doch dein Leiden. 87.

1. KanoK ânianitit, Pillorigutiksakka, Pivlugit, Jêsuse, Nakorijæksarivagît? Illale illûnapkut Perkutaulanga illingnut.

2. Ômattiga aumavoк, Jêsus erkaraigupko Nerkerksortitaunît: Sorlo pijaulauravit Issakajaudlarlutit, Taimak pinnaridlarpagit.

3. Ômattima illale, Opigijæksarivâtit Kugviudlarnermut, Igvillo aunârtotit,
Ungagijomavagit, TækkoKârnanga kênarnik.

4. Jêsuse! tussartigut, Tuksiadlaraptigit Angervigivlutit: Ikkingnêtsomavogut, Aniallo inungnut Okautigijomavavut.

\section{Mel. 127.}

O Lamm Gottes unschuldig. 88.

Passijæksaungitotit Tokкojotit Kejungme, Sinnaungaitsainartotit Nachogijaujotille;

Pêjangikungne ajornivut Assionajalaukpogut; Nâpkigitigut, Jêsuse! Jêsuse!

\section{Mel. 32.}

Wenn ich den Heiland für mich. 89 .

1. Piulijiga âniadlartor Anernipkut tautukôlerupko,

Ômattiga kiksadlalerpor tagva,

Missigigapko âniajuksaunera.

2. Illa Kuviasudlalerpunga Erkaigama Piulidlartima Aungminulle pissiaridlarmanga,

Ikkiminut ajorungnaitimmanga. 
50 Kristusib ânianinganik tokkunganiglo

\section{0\%. Mel. 14.}

Dein Leichnam blutet jämmerl.

1. Timivit aunga takkarnit Tamainit kôlerpok, Tamâle ômattimnuttauk KôlitsainarletôK.

2. Piulijiga, pivlunga Âniadlaravit, Kanorle piniarlunga Akkiniarlutit?

3. Akkinermik ajorpunga, Kaujimadlirparma, Illingnulle ômattiga Tunnijomavara.

\section{Mel. $151 \mathrm{a}$.}

Nach tausendfachen Plagen. *128.

Tarnikut timikullo

Âniarârlutit

Pivlunga, kejungmullo

Nivingartauvotit;

Ajornerijakkalo

Pêjartaukovlugit

Ikkingnut âkigamga

Kujagidlarpagit.

\section{Mel. 165.}

Jesu, deine tiefe Wunden. 90.

1. Jêsuse, kiksarangama Tarnimne timimnelo,
Mannigotsainarpângale Ikkivit tokKovillo; Koksalailerangama, Âniat erkarapko, Ajortomut tamattoma Mittagungnaitilerpânga.

2. Kiglormulle ajornima Kajungersalerppanga, Tokкut erkaumalerapko, Tagva pijungnaipunga Sâtanib ôktorppanga Ikkitit tâpsomunga Kemmergojautinapkille Kemmaksâdlalerpângale.

3. Okpingitut ômattiga Asseroromarlugo Illaukojomalerppanga Ajortulliarnermut, Âniatille tagva Ullernaigidlarpakka, Tâp Idluitullerкonnanga.

4. Ajornikkalo tamaita Pivlugit kiksaruma, lkkivit mannigorpânga Tokxogavit pivlunga; Taimak nekkorsivunga Inûlijaulerama, Illumne ikligungnera Âniavit tokkungmago.

\section{Mel. 79.}

0 Welt sieh hier dein Leben. 91.

1. Illagêktut okorse! Tækkojartolersiuk 
Inôtigijase!

Tokrotaujoк kejungme,

Âniadlartauvlune

Gûde Nalegaujotuar.

2. Nautsertutsiarsiuk

Timinga aunâdlartor

Ikkilertaudlartor;

Ômamigut âniamut

Serlekititaulimut

Angijomik annersârtor.

3. Kia taimak pivâtit?

Âniatidlarlutit,

Næglingnadlartotit;

Ajortorangitotit

Inuktun-inginavit,

Asserorsimangilatit.

4. Illa uvanga tagva

Ajortullilaurama

Âniatipagit;

Idluitullinimnut

Pitlartautilaukpagit,

Ajornima nênermattit.

5. Pitlaræksatôvunga, Aggapkut ittikapkut Kellaksortauvlunga, Egitæksaudlarpunga Kappianartovingmut, Âniatigivarmale.

6. Pitlartaujutiksakka Nênidlartiksakkalo Illingnut pivattit; Pijauleravit taimak,
Pilloriktilerparma,

Piuligamga tâktomit.

7. Tоккоmulle aijotit,

Errsinartoksoarmit

Piulilerlunga;

Tokkotæksaudlarnera

Pêjautiksarsidlarpat,

Tokкomut naglilauramga.

8. Kanok Gûd ajungitok

Illungertûtimmatsuk

Ajortullinipta,

Erningat ânianga,

Issakajauningalo

Nautsertudlaromavakka.

9. Akkilêvigilutit

Sappidlarama sumik,

Tuksiarpagille:

Tokкut âniatillo

Ômattimnêtsainarlit

Tarningêrrkârtinnangale.

10. Âniat Jêsusiga

Erkaumajomavara

Nanêkumalônêt,

Taimak erkaitiparma,

KanoK nagligigamga

Kejungme torkomut aglât.

11. Piulijiga illa

Kibligutitiparma

Âlunga uvamnik,

Tarnigalo timiga

Kaidlalerpakka tagva,

Âniarnut piglerkik. 
12. Aungmik Kugvioravit, Annersâdlarmigavit, Âniadlarlutit;

Aijomârpat tarniga Aggangnut Jêsusiga Angergarviksara pikpat.

\section{Mel. 165.}

Tautukôrpagit tagvane, Nagliktiga Jêsuse, Tarniga piuligangne, Tokкogavit kejungme. Kujagidlarpagille, Najutsainarlagillo Kikkiaktorsimavingne, Piniaramga tagvane.

\section{Mel. 165.}

Sei mir tausendmal gegrüsset. 92.

1. Kujagênaromavagit

Nagligitsainaramga;

Ajornikkalo tokkungnut

Pêjatsainarlaurangne;

Illa kibliktauvunga

Sêrkortolerangama

Kejungmele toKkovingne,

Piuligamga tagvane.

*2. Kittiksaungitut ikkititi

Ômamut erkipakka, Aullo kussertox timingnit Kikkitsat inninginit.
Inuit neliata

Ipaksijungnarkagîk Immerungnît, rềmitingnît Inuit piulivlugit?

3. Piulijik! inûlinga Asserorsimanimnit, Ajornikkalo tamaita Pijakka sivolimnit, Idluilutakkalo

Pêjakit, piulinga; Aungnut serparsalerumga, Ajorungnairtitauvunga.

4. Jêsuse auggit ikkitit, Ômattimnut aglakit, Illumne uvlut tamaita Issumagênarlugit. Pituaridlarapkit, Omattimnêtsainarit, Ittiggangnut ingilanga, Nagliklutillo pilanga.

\section{Mel. 168.}

Jesu moines Lebens Leben. 95.

1. Inôgutiksarsijiga,

Tоккuksaerudsigalo,

Tunnilauktotit pivlunga,

Kappianadlartomut, Ânianadlarnerpâmut, Tоккокојоmanginamga: Opigomut Jêsuse Kujagidlarpagille.

2. Jêsuse âtautipâtit Mittautigællutillo, 
Ketserarlutit pivâtit

Issakadlarpâtillo;

Taimailijautigivarma

Tokкomit piulivlunga;

Opigomut Jêsuse

Kujagidlarpagille.

3. Ômamut kujagivagit Âniadlalauravit,

Ikkilertaudlarmat timît,

Pivlunga tokkogavit,

Tarnikut kappiasumut

Kiksamut sajulauravit;

Âniat sorairnanga

Nakorijomavara.

\section{Mel. 126.}

Wenn meine Sünd mich kränken. 96 .

1. Ajornikka pivlugit

Kappiasukuma, Illingnut Jêsusiga

Issumakarlanga ;

Tokкolauktotit kejungme,

Ajornikka tamaita

Pêjartaukovlugit.

2. Tattaminiarpunga

Erkaumalerapko:

Nâlegak âniavok

Uvanga pivlunga;

Illâle pingortitima

Tokkotigidlarpânga

Assiokonnanga.

3. Tamannale pivlugo Illa Jêsusiga
Inôtillunga mâne

Kujagidlarpagit,

Pivlunga âniagavit,

Tokкogavit pivlunga

Nagligilauramga.

4. Nâlegax! âniavit Kajungersarlinga, Ipperarniarlugit Ajortullinikka; Erkaumatsainarlagole Akkitojotuamut Piulilauramga.

5. Sorlo piniaravit Uvamnut Jêsuse ! Taimak Kattangutimnut Piniarlangale,

Takkomôrnermut pinanga,

Nagligosungnermulle, Sorlo pilauramga.

6. Kingorlermik ikkivit

Mannigudlarlinga, Stundima kingorlingine! Kollalernangalo : Tættituarigapkille, Illalliolâramga Taututsainarlutit.

115. Mel. $184 \mathrm{~b}$.

Wenn meine Seel' den Tag bedenket. 97.

Uvlox taimna issumagigupko, 


\section{Kristusib ânianinganik toккunganiglo}

ToKkungmat inûlijiga,

Ânertaungmallo, ikkiminut

Inûlivlunga ; Nâlegar!

Kugvinut tuksialauravit

Omigijitit pivlugit,

Pivlungatauk Kugvioravit,

Kennuvagit, nâpkiginga.

\section{Mel. 51.}

Da Jesus an dem Kreuze stund. 98.

1. Jesus kikkiaktortaungmat Timinga ikkilertaungmat. Âniataudlarlune, Kingorlît Jêsub okausingit

ErKaumadlarlavulle.

2. Sivorlermik Atâtane OKaupâ: illiorningit Issumagijungnaikit; Nellungmagit piniarnitik, Tоккоtileraminga.

3. Aipânik, inuartomut, Nâpkininga erkarsiuk, OKautingmago illa, Uvlome nâlegauvimne Aipariniarparma.

4. Pingajuanik illaminik Issumagimut okarpok: Marîa ernît tagga! Johannes anânat tagva! Pattangaitiuk tâmna.
5. Sittamanganik niplerрок, Kappiaktoadlarlune, Tarnimigut âniamut: Gûdiga! Gûdiga sôngme Uvanga Kemmakpingâ?

6. Nâlegavut xêlerame Tellimanganik okarpok: Immerosudlarpunga! Pivluta serkomitaungmat Inôtigidlarpavut.

7. Pingasojoanik oкarpoк: Mânna tagva nâmaksivoK! Suna? piulijiksak. Sumut piulivâtigût? Aungminut kovijomut.

8. Kingorlermik nagliktipta Atâtane kaigarsukpâ: Anernera aggangnut Perkovara! niakone Nairpâ, tokкolerlune.

9. Tagva Gûb ânianganik Ivlersijok, nagliktorlo, Ôma okausinginik, Kuviasûtiksakarpoк Inôtigigamigit.

\section{1\%. Mel. 9.}

Endlich nahm man ihn vom Kreuz. *138.

1. Unnulermat, kejungmit Jêsus akkartipât, 
Ikkiliksoar timâ

Illuvermut âpât.

2. Jêsusiga pivlunga, Anernangêravit,

Nunamênimne tamât

Tættigênarlagit.

\section{Mel. 185.}

Christe, du Lamm Gottes, dein Versïhnen. 99.

1. Kriste, Gûb sauganga âniavît

Ômattiga kiblikpâ;

Opigomut reavuk ijîkka,

Tautudlaramittille,

Âniajotillo tokkojotit,

Kejungmut kikkiaktortauvlutit;

Taimak kibliktaunermik

Tuksiarvigivagit.

2. ToKkovit manniguidlarninga

Nekkorsitigilugo,

Tarnipkut kikkiaktortauvingne

Najutsainarlagillo ;

Piulijiga nellungilarma, Tokkungnik nellojungnaitinnamga,

Mannigortaumagama, Taimaitsainartiminga!
119. Mel. 151 a.

Du, der sein menschlich Leben. 104.

Jêsuse tunnigangne Inôtsit tokкomut, Pivluta naglingnermut, Uvagut Gûdemut Ullapkutekarkovluta; Kanok tagva pitâ? Akkinniarlutille Naglikavit taimak.

\section{Mel. 184a.}

Kein Tröpflein Bluts war dir zu teuer. 105 .

Aut illunât ivlerinago, Pivluta kovilaurangne, Naglingnivit tarnivut mânna Ônarsidlalerligille! Âniarniglo toKKungniglo Missigititigut tagva Illuptingne uvlut tamaita, Annernangêtrârtinnata.

\section{Mel. 168.}

O Behold your Saviour wounded. 112.

1. Kemmergosiuk Gûdivut! Kejungmut nivingartak, Naglingninga uvaptingnik Ipaksinatsangilak, Tussarsiuk tuksiarmat Ômigijine pivlugit: Atâtak nâpkigikit Pijatik nellungmagit. 
56 Kristusib ânianinganik toккunganiglo

2. Kikkiaktortaugamelo

OKausingit erkarsigik, Johannese Marîalo

Issumagigamigik;

Okaramelo : Gûdiga

Sôgle mânna kemakpingâ?

Jêsuse, nâmaksivok!

Okarame, toккорок.

\section{Mel. 243.}

Dem heiligen Blut des Herrn. 106.

1. Jêsusib aunga kovijor pivluta,

Erdligilavut opigilugo,

Kissiallo nâlekpavut

Ômungale ubvarmattigut.

2. Ikkingit pivlugit nertoromavarut,

Tâpkonungalo inûlingmattigut,

Toкkomillo piulivluta. ::

3. Jêsuse aulik, erkitsomavagit,

Aggut kuttersuit immerlugit,

Pivlunga igvit aunârtôgavit. :,:

4. Kiaptikarnît aullo kussilerle,

Tarnivullo saimarlugit mânna,

Ubvarlugit ômattivut
Ubvakit aungnut $\overline{\text { kovi- }}$ majomut.

\section{Mel. 64.}

Fünf Brünnlein sind. 107.

1. Puailavît tellimauvut,

Tâpkonangat pivakka

Kuviasungnek, mannigornek,

Nekkorsijutiksakka.

2. Ajoruma kannimamut, TokKublo tikkippanga, Kingorngane, tâpkonane Merngoeromavunga.

\section{Mel. 151 a.}

O Haupt voll Blut und Wunden. 108. 109.

1. Niakoк ikkilênar, Mittautigijaujor! Niakôsertaumajok Kakkilautilingnik! Gûb ânanauningane TakkuksaurâlauktoK, Mânnale rataktitok, Opigidlarpagit.

2. Kênavit tautuanut Silla sajuktipat; Mânnale pinnarnarnît Nachogijaudlarpok, Ketserartaudlarpotit 
Kattaktilutillo;

Kia taimak pivâtît

Pitlarmilutillo?

3. Nâlegar ajornima Âniatipâtit;

Pitlaræksauvungalo

Tâpkoa pivlugit.

Ahâk ovanêpunga,

Ningarijæksaulunga;

Jêsuse Nagliktiga

Nâpkigilaungminga!

4. Najoromadlarpagit

Nachoginiarnanga!

Ômattimne nutâmik

Sakkervigilaunga,

Sorlo tokrolertotit

Naingalertillutit.

Allarungnailerpagit

Ungagidlarapkit.

5. Sakкervigilaungminga!

Tokкonialeruma,

Tautukôromavagit

Tokkotigigamga.

Pituaridlarpagit

Piulilauramga,

Erkisimiarlagit

Ômatimnut mânna.

6. Ômatimne tagvane

Kujagidlarpagit,

Tarnikut âniagavit

Toккolertillutit;

Illingnêtsainarlanga,
TækkoKârtinnanuk, Aggangnit pêrroonnanga Nunamêtillunga.

7. Aungêrotileruma, Najulaungmingale; Tarniga neksarlugo Kưianartovingmut. A nernernut timiga Innigilaurangne, Uvlullo naggatâne Omartilârparma.

\section{Mel. 22 a.}

Mir ist's, als ob's Charfreitag wär'. 110.

1. Nâlekama tokкovine Uvamnut illingatipâ, Sorlo pivlunga uvlome Kikkiaktortaungmat sâmne.

2. Nivingajorlo kejungme Tautukapko anernipkut, Kemergogapkolo sorlo Johannesib Marîablo.

3. Sorlo sorsuktuksab mânna Angmarmago senneranga; Ómangat auglo immerlo Ajornimnut kôktipakka.

4. Gûde taimaitsainarlanga, Siorniornît pivlunga Ômame serrigilugo, Inôtituarigapko. 
126. Mel: 23. Aria. Ave, o du blass' und bleiche. ${ }^{*} 160$.

1. Ave, rattaktitotille Giûdib inûblo timinga! Ave, aungmik kiæguktor! Ave, timâ illijaujor!

2. Kugviorlunga illumne, Jêsusib illuvingane, Tagvanêtsomavungalo Tikkitsearilug olo.

3. Tagvanêtsomavungale Jêsusib illuvingane Aungerotijok timinga Ullernaigijomavara.

4. Ilia timiga tagvane Merngoêrseromavorle, Kêmitigijomavara Angergalerkârtinnanga.

\section{2\%. Mel. -58 .}

Meine Leiche Jesu. 113.

1. Kattaktitor Jêsub Timinga piga! Sorlo kissima piksarijara Illuvertak.

2. Aungêrotor time

Piksarivara

Sorlo illa uvanga kissima Pigivara.

3. Pinnarnartotuak Jêsub kênanga,
Kanga kênarma adsigivaûk?

Illuverme.

4. Saimanartut aggait, Jesusib pigik

Tammarmil kikkiæktortaunigik

Kunnikpapse.

5. Nagliktok Jêsuse Tikkimiguvit,

Keajungnailerpunga tagvainak

Tækkogupkit.

\section{Mel. 240.}

Ave, Gott Schöpfer mein. 115.

1. Pingortitigale!

Nertorpagit mânna

Aungnut piuligamga,

Pissiarivlunga

Tokkotigamga ;

Kujagidlarpagit,

Kikkiæktornernik

Taimaktauk ikkingnik

Erkaitsainarlanga,

Inôtillunga,

Nagligidlaramga.

2. Tagva sorairnanga.

Kuviasukpunga,

Tamainiglo pimnik

Tunnijomavagit,

Nâlegomagapkit; 
Aungnullo tokkungnut

Tamaitale uvlut,

Ômattimnêlaurit.

Illitarivarma,

Ajulerama,

Najungikumga.

3. Jêsub timinganik

Kunikpallaivugle

Jôsef Nikodemlo

Illijartoramikko;

Taimaktauk Marîab

Kugvinut kunnikpait

Aggangit ittiggangit.

KanoK ikpigivât?

Tâpkoa Johanneslo

Jêsub timinga

Tækkonâramitsuk.

\section{Mel. 109.}

Ach wiederholt mir Jesu Leiden.

Aulajeкattautigilakka, Jêsub âniâ tokroalo, Taimak ikkingit auggit. Illutorsimajuglo ijîk, Ômattâlo angmartitaungmat,

Pivlungalo tarningêrmat.

130. Mel. $184 \mathrm{~b}$.

Ich seh in bangen Buss-ideen. 117.

1. Illanârijama tarninga, Âniamut kiksadlartoK;
Pivlunga tautukôrivakka

Ijîglo kugviudlartuk;

Okpinginimatauk ômame, Illûnatik ajornima

Siorniortipât taikane, Piulijomadlarmanga.

2. Aileruma perorsêvingmut Jêsusiga tautuklutit, Kênat nutsattillo kussertut

Aungmik kidsijâleravit; Keagavit kappiasumut, Senningne tuksiarpagit: Ômattiga kusservigiuk, Pivlungatauk Keagavit.

3. Illungertudlartoк tоккоmut,

Illuêngartor âniak,

Engelit illangata tikkitub Nekkorsitipâ takpângat. Ermgusiarsuk anialik Ajornangipat pêrlugo! Atâtaminut tuksiartub Kiblikpânga tussarapko.

4. Ketserartaujok kênangagut,

Mittautaumut tigluktaujoK ;

Kiglissiniartut sânginut Pivlunga nangertitaujok. Kakkilautinullo âniak Niakôsertauvlunelo

Kollêngalo nerkersudlartor;

Tautukapko aumavunga. 
5. Erksuklugo senningajolik, Aivor mânna kiksarlune, Ômame ikpigijarêrpor Âniajuksaunerminik.

Taimak Kannilalaurapkolo

Tikkilunga Golgatamut.

Tautugvimnut nivingartamik,

Tagva illuga keavor.

6. Aggangillo ittiggangillo Angijomik âniajut;

Pivlunga puttortaulermatta

Kugvinut kunilerapkit, Sennerâgut kappijaulerpor;

Âniane sorêrmago:

Sillaksoak sajukpilakpor. Saimarsautiksara kôkpor.

7. Aungmik immermiglo kôngninga

Ânanaujok, opigomut Nelloprotimullo uvanga Pivlunga erkaumagapkit, Âtsilerlunga ômattimnik, Tuksiarlutit: ômunga Kusserle soraijuitomik, Nuname inôtillunga.

8. Ânianga ômanartomik Aglagletôk ômattimnut, Kênamnelo tækkuksaulaulik

Ijîk illutorsimajuk;
Aklunimnik sangênimniglo

Kaujimadlartotôgame, Tâpkungninga oxautigupko

Mannigotsainarpângale.

\section{Mel. 185.}

Für uns ging mein Herr in Todesnöten. 118.

1. Tokкub kassilininga Nâlekab

Ikpigivâ pivlunga ;

Perorsêvingme tuksiadlarpok

Kuviasugvilksamnik;

Pivlunga sajulerpor tagvane

Tarnimigut siorniorame;

Tuksiarmut kênanga,

Kattaktipor pivlunga.

2. Pivlunga kappiasulaurame Aungmik kidsijalerpok, Nunamut kussermat, Engelible

Nekkoksijartolerpâ;

Pivlunga timimigut sajukpok.

Ijik kugviornermut puvipuk,

Kênâlo næglingnartoK, Innerkonarungnaipok.

3. Pivlunga taimak tigluktaulaukpor 
Okautaujungnangimat;

Omattiga ônarsidlalerpor Tautukôjarangapko.

Ahâgle kollêngata killingit!

Tækkosiuk kaunga ônadlartok!

Niakub âniangata

Kiblikpâ illûnara.

\section{Pivlunga kikkiaktortau-} viksane

Erksukpâ Kenuêdlune,

Saugartut uvlune taipkonane

Aijotut torkotauvingmut: Pivlunga ômattinga Kagvarpok

Timingane, ônadlarnermulle;

Pivlungalo êsivok

Kêlerdlune sungarmik.

5. Igvit kênak annerimajotit,

Kattaktilertillutit!

Kanortôk taimak remergolagit

Akkinnijârvigilunga!

Illinganît Kejungmêtillu-

$$
\text { tit, }
$$

Tækkolauktavut anerniptigut,

Pinnaridlalerpavut, Assinginit tamainit.
6. Ijîkka kemergoleritikko

Niakor kattaktitok;

Tokrodlune naingalertor mânna,

Inunguut ajortunut!

Omatîk attavigitsainaruk Tarnîk ikkingit ungagidlakit!

Ijiglo illutortuk

Puigorniarnagik!

7. Tussarnernersaujokarniarane

Siutimnut nuname, Ânianginit tokkunganillo, Inôsimne illûnâne.

Tuksiarningatalo nippinga,

Annersauminingillo tamaita

Tuppaktitaujutiksamnut Tussatsainarlaulakka.

8. Nâmaksidlarpunga pigiÂniajub tautua, Imgerutigingungilaralo, Tokkolerkârtinnanga. Ômattimale nertordlerningit

Mikkidlarput sulle tâpsomunga,

Ômattâ ônadlarmat Naglingnermut uvamnik.

9. Taimaimat ômat nellekangitotit, 
Tautuit tokkotillutit,

Nippaktuksaungilak ijiptingnut

Taututsainarkârtinnæk!

Jêsuse tokkotigigaptigut, Merngortorniængilat torkluvut

Nakorijomavlutit, Paunga perkârtinnata.

\section{Mel. 151 a.}

O süsse Seelenweide. 119.

1. Nekkoksinarput illa Jêsub âniangit! Opigosudlarpogut Tautukôruptigit Jêsus, anerniptigut, Kunnutuidlutit Tokkovingnut aigavit Kissipta pivluta.

2. AiparilaulagittôK! Getsemanemelo Pivlunga kiksarlutit Tauturôlaulagit! KanoK tagvane timît, Merngortortor pângmangmat,

Aungmik kiægukavit Ullapirsautimnut.

3. Tagva tækkolerpunga Pitlaræksaugama: Jêsus nâpkigijiga Tunnivok pivlunga.
Aungmik kiægungningat. Tarniblo kiksartub Kaujitipânga illa Ôma naglingmanga.

4. Jêsus annernangêrmet, Uvanga pivlunga, Erkaumalerangapko Kugviorpuk ijîkka, Allaromangilara Pivlunga aunârtok, Ikpigingikupkole Illuga tokкovor.

5. Âniadlarkârving nit Siornioravit, Kallugiaksoarmut Kappijautillutit Tautukôtsainarlagit Innôsimne tamât; Âniatit tamaita Inôtigællugit.

6. Tokкojotun-îdlunga Tiguvarma igvit; Kanoktôk ômattimne Nakorênarlagit! Opigidlarlagillo Sujungaitinamga ; Ajutsainaramale Ikkingnut pairinga.

7. Illa nægligivarma, Nagliktor Jêsuse! Johannesetut tagva Erdligilaulagit! Sângne Kugviorpunga 
Nellopкotinermut, Kangusumullo illa Naglingnikinapkit.

8 Ikkingnêniarpunga, Illa nagliklugit, Nautsertorluarpakka Tamaitale uvlut; Ittiggangne-ilanga Nunamêtillunga, Kaikole'kârtinnanga Illingnêkovlunga.

9. Tarnipkut Jêsuseme Itsainalerpunga Âniangat illumne Mannigudlarmanga, Kemmergotinaminga Ikkersoarminik, Okaupânga : ômunga Piulijauvotit.

\section{Mel. 9.}

1. Kristuse, Gûb Erninga, Âniat pivlugo Najortigut tamapta, Tokkungnut aungnullo.

2. Tokkotigigaptigut Omattivut pikit, Perkovavut illingnut Pissiarigangne.

\section{Mel. 124.}

0 dass ich bis in mein Grab. 120.

1. Kannoktôk illuvimnut, Jêsusiga!
Pivlunga serkomingmat tokkungmallo,

Inôkovlunga; tâmna erKarlago,

Ungagilagolo.

2. Ômattimne tokkonît najorlago,

Tamât siorniornît,

Kaut tamâllo

Missigilakka, aungnut âKiklunga,

Tokkotigigamga.

3. Tækkolaulanga Kanoк Jêsus timît

Kiægulaungmat aungmik, kappiasumut, Kanorlo Kugvinitit

korlormatta

Pivlunga nunamut.

4. Ama tækkonâlaunga

perorsêvingmit!

Tagvunga tautukama; taimaitunik

Pijokalautsangilax, inukulluk

Piulivlugole.

5. Merngoêrservingnullo timigale

Illivara, Gûd timît a đầgilugo,

Tagvungaromavunga Marîatut

Kugvioromamut. 
6. Akkilêvigilutit, Jêsusiga,

Âniagavit, piuk inôsera, •

Illuvimnit timiga Adsigilutit

Ômartaukârnago.

7. Timigale illiktun itsainarle,

Ômattiga illingnut killanarle;

Angergarkârtinnanga illingnêle

Inôniarlanga.

13. Mel. 185.

Blut'ge Leiden meines ein'gen Freundes. 1.21.

1. Illænâtuarijama Gûdib Aunârtub ânianga, Tautugangapkole ômattipkut

Ônarsititauvunga,

Nekkoksijaunek inôsimnelo

Sokкoserkojomatsanginapko;

Tarne Getsemaneme Golgatamêtsainarle!

2. Jêsub kikiaktortauviane Itsainaromavunga, Kugvinut rujagilugo Gûde, Ikkingit kunniklakka,
Pivlunga illutorsimangmanik

Tautuklunga kallugiartaungmat

Auglo immerlo kôktuk Naingalermat tokкomut.

3. Tokkovit kiblikpâ ômattiga

Sakkervigilauramga;

Aiparingalo uvlut tamaita Mattomanêtillunga,

Tarnivit siorniorninganik,

Tokкungniglo illumnut aglagit;

Illuvernêlauravit

Innôtigidlarpagit.

4. Kattaktitor auliglo Jêsuse! Ômattima angmartub. Immeruktotut tikkipâtille Aungmik immertilauruk, Niakungnit korlorsimajomik,

Tamainillo ikkingnit pijomik ;

Nekkorsijaulerlunga

Sulle ajoksarama.

5. Tagvane kemmergojomavara

Jêsuse nagliktara;

Timingalo tokкојок pivlunga

Tarnipkut tautukpara; Jêsub ânianga tokkungalo, Ânanâvaksuit ikkingillo, 
Jêsub timâ aungalo

Pituaridlarpakka.

6. Nâlekamalo illuvinganut

Sinnigiartorpunga;

Tarnimnullo erkaumalerapko

Nekkoksivor illuga.

Unnugangat, Jêsub inningane

Timingat, merngoêrserviane,

Tuksiarvigigapko

Sinnitsiarpungalo.

136. Mel. 151 a.

Ich bin in meinem Geiste. 123.

1. Tarnipkut itsominak

Jêsub najugâne,

Ikpigiluarvimne

Mannigortaunimnik;

Tagva tækkotipânga

Anianerminik

Okautilunga: takka

Pillorigutiksat.

2. Pâmaktervigivara,

Opigilugolo:

Tussarkattarpungalo

Piniarninginik,

Pivlunga; ômattiga

Unarsititauvok,

Tâpsoma ajornikka

Tokkotigingmagit.
3. Jêsub illuvingane

Itsainalerlanga!

Kusserviolangalo

Missuksoanganut

Timinganit pijomut

Ikkeliksoarmit;

Kiægungnerit auggit

Kôgvigilerlinga!

4. Tagva ovanêpunga

Illanârijamne,

Kugviorutigilugo

Kujalidlarnermut.

KanoKtôk ijîngmale

Sânganêtsainarle!

Tagva nâmaksivunga

Illa sorairnanga.

\section{3\%. Mel. 79.}

Ich wünsch' mir alle Stunden.124.

1. Kaut tamât, ômattimne

Jêsusib ikkingine.

Inniksarsilanga!

Aunga opigilago,

Âkitsainalermanga,

Ungagijomavaratauk.

2. Ajornikka pivlugit Siorniudlarningit, Pitlartauningillo, Erkarupkit tarnipkut; Ungagijomavara; Kujalimut aumavlunga.

3. Angnerpâk mittautaungmat 
Tautukapko tarnipkut, Ánanaugivara;

Kugviorvigilugo

Merngortutsænnangalo

Kiblidlarmago illuga.

4. Allagaigilaukpakka

Erkaumajaujungnartut

Tamaita, tarnipkut;

Kibliktaulaunangale, Tikkilerkârtinnanga Golgatamut. Opinarmêk!

\section{Mel. 167.}

1. Âniatitaugaville Aungêrotilerpotit, Pitlartaunit soræmalle Merngoerserluarit; Kugvimnik mitsuktipakka Ikkitit, killitillo, Timît ânanaugivara Nellipsajarangapko.

2. Merngoêrserle sinnikle, Nunamut illijaujor; Kiksadlartor tarnimine Токкоmut pitlartauјок; Aiparilerpâ tarnima Timâlo kemangnago, Allaromangilarale Токкокаttigilugo.

139. Mel. 208.

So wie eins im Bette. 174 .

1. Sorlo merngortortoK Merngoêrrseromamut
Sinnigomangmat, Taimaktauk tarnima Jêsusib ômattâ Najoromavâ; Ômunga rêlerpunga, Aunganiglo tokkunganik Missigijomanut.

2. Jêsub illuvingane Sunakadlarnera Timâne illa Taijungnangilara. Tamânênarlanga! Nekkoksinarmat. Mâne tarnima illa, Tamaita ânianingit, Kemergolermagit.

\section{Mel. 14.}

In meines Herren Tod und Schmerz. 125.

1. Jêsusib âniangane Inôluarpunga, Ômattiga killanârmat Tâpsoma aunganut.

2. KanortôK inôtillunga Erkatsainarlago, Tokкungallo kiblêninga. Ikpigênarlugo.

3. Auktojor piulijiga Uvlâmit unnungmut, Âniatit ikkitillo Ullernaigilakka! 
4. Kanoktôk tautukôrlago Tokkonît kejungme, Kênavillo kidsijârninga Ullernaigilago.

5. Mânna okpervigigapkit Erdligilutillo, Illa tækkojomavagit Nertorlutit tagva.

6. OKauserlo kingorlerpâk, Naingalaukârtinnæk, Tussaromadlalauktara Kejungmêtillutit.

7. Tagvanêtsomalauktunga Tækkonârlungalo, Kallugiamut ômattit Kappijautillugo.

8. Uvlut nelliutikpatta Angergaleruma, Tarniga aijomârivat, IllingnêKovlunga.

\section{Mel. 208.}

Lamm, du hast geeilet. 126.

1. Nagliktiga! tagva Inûlidlarparma Missigivunga;

Nagliktainarnanga; Golgatame illa Naglilaukparma, Tagvane, naglingnivit Sêkpâ sorlo ômattille Naglimut uramnik.
2. Jêsusib sângane Erkailerumale

Kugviornermut, Kaikolaungmangalo,

Kuviasukovlunga Inôtillunga;

Tagva kujagivara Ittiggangillo puttoggit Erkitsomavakka.

3. Ômattimne tagra Killanâlerpunga, Ânianganut; Issumagigupko, Keasôngovunga Kujalinermut. Jêsus tuksiarpagit: Tarnigalo timigalo Ajorungnaitikik.

4. Omat kap

Tarne âniajok!

Time sajuktor!

Tarniga timiga

$\mathrm{P}$ iulijaukovlugik

Pijæksanginit. Ômattiga ônarpok Kujagilugo, takKakka Tiglilerangatta.

\section{Mel. 146.}

Ich halte meine Fahrt. ${ }^{*} 177$.

1. Jêsub timinganut, Ungatsainarpungale; Erkaumalerapko 
Ijîkka kugviorpuk;

Kanneralo mânua

Nippangeromavor,

Nâlekab ikkingit

Killanârigapkit.

2. Pissiarigangne

Tarniga, Jêsusiga!

Piniaramgalo

Assiokominanga,

Kugviortuk ijik,

umallo ônartor

Uvamnit pigikit

Unêt ajortunga.

3. Nâlegar pivlunga

Imâgle tigungmatsuk,

Mittautigilugo

Tautukpara tarnimnut;

Annaudlarivâlle,

Kênanga ketserarpât,

Timâ ikkilerpât,

Aungelertidlugo.

4. Aungêrotor time!

KanoK tainiarlagît?

Pinnarigapkille

Pinnarnartunit tamainit.

Jêsus nagliktiga!

Tuksiadlarpagit:

Timingnik aungniglo

Illumertilaunga.

\section{Mel. 151 a.}

Gûdiga Golgatame Anernangeravit
Tamaita nâmaksilugit

Atâtangnut tarnît

Perkogangne, pivlunga

Illuvernêgavit,

Taimak piuligamga,

Kanoktôk pilagit.

\section{Mel. 185.}

Wenn ich Jesu Grab im Geist. *178.

1. Anernipkut Jêsub illuvinga

Nellipsajarangapko,

Tagva inôgutiksarivakka

Ikkingit âniangit,

Sennerangalo innigilago

Tokkungalo nangminerilago

Nunamênimne, tagva

Kanoêtsungnaipunga.

2. Tagvanele illænnârijara

Tækkonârungnarpara;

Anernipkut tâpsoma timinga

Tautukôtsainarpara;

Jêsusib kikkiæktortauninga

Anernangerninga ikkingillo,

Timingalo aungalo,

Pituaridlarpakka.

3. KailauritsetôK tækkoluselo

Jêsub ânianginik!

Kemmergojauluarungnangitunik 
Inûb kittorganganut!

Mâne nekkorsijutiksakarpoK

Soraijuitunik ômattemut.

Kêmitigilavuttôk

Marîatut illuverme!

\section{Mel. 99.}

Ach wundenvoller Martermann. *179.

1. Ânialik nagliktôjor

Kaunganillo aunâdlartok,

Kejungme niakone nairmat,

Kingorlermik annersârmat, Tarne timânit anningmat, Illutorsingmat kattaktinginat.

2. Kugvinut tautukpaptigit, Ajornivullo pivlugit Jêsuse, nivingartaugavit, Illijartortaugavillo;

Tokkomit makkinavillo

Idluarsijutiksaptingnut.

3. Anernangêrtok Nâlegax! Merngoêrserpotit mânna Illuvermut illijaugavit. Mannigorniksarsivunga Ânianerilauktattit Nellikârlugit erkarapkit.

4. Atâtavit agganginut Anernît perkolaurangne,
Ânianitit naggaserput.

Timît ikkilersimajor umamut tækkonârlugo Inôsera nalaulagotôK!

\section{Mel. 151. Aria.}

Da sind wir arm und blöde.

1. Jêsus anernangêrmat

Uvanga pivlunga, Erkaumalerapkole Kugviudlarpunga; Ânianga tokkunga, AungêrotoK timâlo Nunamêtillunga Pituarivakka.

2. Illingnut okperpunga Erkidlarlutillo, Tokkogavit pivlunga, Illuvertauvlutit; Jêsusele âniat Tættigivara kissiat, Angergaleruma Illingnêlârpunga.

3. Kiksarvingmêtillunga Ullapirsautingnik Missigitilaungminga Taimaktauk tokkungnik; Kilangme sorairnanga Nakorijomavara Aniat, pakkoa Illagilugit illa. 


\section{KRISTUSIB MAKKININGANIK ILLUVERMINIT.}

14\%. Mel. 114.

Halleluja, dass Christus wieder lebet. 140.

Hallêluja, Kristuse ômarmingmat,

Nâlegak nâlegauvingminêtok

Uvlunik pingasunik toKKојов,

Tamainullo mânna nâlegaungmalle,

Inuit tamapse nertorsiuk! Piluaritse inukotingit.

\section{Mel. 132 a.}

Kommt, betet an bei Christi Gruft. 129.

1. Perkutaujose Gûdemut

Opigomut aigitse Jêsusib illuvinganut; Mânna nertordleritse, Jêsus illuvermêlaurtor Illuvermit makkimingmat ToKKо sâlagilugo.

2. Nâlegara Jêsusiga ToKkomit makkinnavit Inukotitit tamaita Tokkomit kelluvattit; Tamapta piuliklernernut Ittertitaulerkovluta Kuvianartovingmut.

*3. Jêsub okpertorotingit Illagêlerput mânna;
Kialo inurotingit Erkartorungnarkagît? Kristus tagga sâlakartoK, Kilangmelo iksivajor Gûdib tallerpiane!

*4. Nertortaulaurit nuname Okpertunut tamainut! Pânelo ânanauvingme Salakarêlauktunut Aungnut piulimajunut! Uvaguttauk, Jêsusivut, Sâlakartingmitigut!

*5. Gûd nertortaule, Gûdivut Sâlarartingmattigut, Tokкomut pijaudlartogut Inôtilaungmattigut. Sâlakarnek tagva piga, Inôngmat piulijiga! Gûdiga nertortaule!

\section{Mel. 16.}

Lebt Christus, was bin ich betrübt. 131.

1. Kristus innôngmat; sôg tagva

Kiksadlartuksauvîgle?

Naglingmanga kia mânna Umangat avipângâ?

2. Saimaner nuisimavor Okpertut opigijangat: 
Kristusele ajugaujor Ômigijiminuttauk.

3. Pivlugo mânna Gûdemut Ningautaujungnaipunga, Pivlungale najugânut Timât avatigingmanga.

4. Jêsus inôgame tagva Serngnigijiga illa, Ômaltima serrigivâ Tokkungajungnaitôjok.

5. Okpervigivagit Jesus Tokкotigidlaramga; Assiojuksauvîk mânna? Taimaitsangilak illa.

6. Soraijuitomik mânna Inukattigigapkit, Ômamut kujagivagit Tættigividlarapkit.

\section{9 a + Mel. 228.}

Gott sei gedankt, dor uns den Sieg. 143.

Gûde opigidlarlavut, Ajugautilaungmattigut, Jêsuse makkilaungmat! Taipsomane, inôserlo Sorsûtingmannik tokrolo, Inôsek sâlakarmat.

Tagva mânna

Kangunartut, oKumaitut Kellaumgujaujut Jêsumut pêjarsimavut.
150. Mel. 228.

Lob sei dem teuern Gotteslamm *197

Nakoridlartaule illa Jêsus Gûdib saugârsunga, Kejungme tokrovlune SerKomitsomalaungmago Kuglugiab niakoa: Makkininga raitsivoк, Mânna-illa - Titsartunermik,

Ajornerit-allarlugit, Kilaklo pijungnarlugo.

\section{Mel. 39.}

Kommt, danket dem Helden. 142. 1. Ajugaujoк kujagijartorsiugle,

Sâlagijarêrmagit ômigijivut,

Inôvlune nâlegauvor illûnainut;

Tamainut tussartaulit nertordlernivut.

2. Tokкo, Sâtanelo sâlagidlarlugik

Piulivâtigut erkartuivingmit,

Ullapirsaumik pititsivor nuname,

Ôminalauktulle pitsartutipaille.

3. Simmêjub akkilerpait akkềngainivut 
Omattivullo aunganut missuktauvut,

Ullapirsartaumavogulle Gûdemut

Adamiblo kinguvângit illûnaita.

4. Attêle nertorsiuk sâlaradlartok,

Piulijauviksavut pijarêrmago;

Inôjotit Piulijivut! inôgit

Uvaptingne tækkozârtinnata illinghik.

\section{Mel. 147.}

Es lebet Gottes Lamm. 144.

Gûdib saugârsunga, Tunnilauktok tokKomut, Inôgutiksaptingnut Umarmingmat tagva; Mânnale ajortogut Kittorngarivâtigut, Gûdib saugârsunga Ômarmingmat illa.

\section{Mel. 167.}

1. Nâlegar kujagilavut Illa ômattiptingnut, Makkimmat tokkungajunit

Nelliutijarêrmat,
Tokkominut aungminullo Piulilaungmattigut, Aungalo âniangalo Opigitsainarlavut.

2. Aulik sennerkab ikkinga Kikkiæktortauningit Kujagênarlavut illa Inôtigigaptigik! Piulijipta tokkunga Missigileraptigo, Taimaktauk ômalerninga Kuviagidlarpavut.

\section{Mel. 15.}

Halleluja, der Heiland lebt. 145.

1. Hallêluja! Piulije

Ômarmingmat, nuname

Ullapirsautekarpogut, Nâlegar nertorlavut!

2. Opigomut pâmakpogut Gûdib najormattigut; Ôma piulingmattigut, Kia passivâtigût?

3. Kina erkartorungnarkâ? Jêsuse tokkolauktor Ómarmat; nâmagijauvor Piulijauviksavut.

4. Kilangmelo nunamelo Nertordlertut imgerput: Nertornak Gûd saugârsuglo, Piulijaumagapta. 
5. Jêsub illuvermêlauktub Âniajungnairame, Uvaptingnut mikkivine Kuvianartidlarpâ.

6. Tokrotigingmagit ôma Inuit ajortuggit, Gûdib ama makkitipâ, Tamanna tunganarpoK.

7. Tunnininga akkitojor Gûdib nâmagingmago, Makkininganut sakkerpor,

Ullapirsartauvogut.

8. Inuk piulijaunerminik Jêsusib tokKunganut OkpertoK, makkininganut Idluarsititauvoк.

9. Toкkomit saukat pairijingat

Gûb makkitinnamiuk, Tâpsoma nâpkigidlarpait Pitlaræksaudlalauktut.

10. Angnerpângojok pairksijor

Aungminik tunnijijor Saukanne pivlugit tâmna Ômarmat, inôvogut.

11. Nertornak Gûd, ajortuggit

Assionajalauktut, Erniangotilermagit Kristusib toKkunganut.
1․ Tættiksakadlarkovluta Nerriunadlartomik, Kingormgujijomârapta Kuvianartotuamik.

13. Makkilaungmat anernermik

Aitortortauvogulle, Ôma nutautipâtigut InôKovluta Gûmut.

14. Gûdib pitsartuninganut Arvertarungnarpogut Ôma nâmagijanganut, Kivgartorungnarpavut.

15. Ômamullo Nâlegavut Nakorijomavlugo Perkojangit mallikpavut Erdligidlaraptigo.

16. Hallêluja nertordlerneк Pile Gûub attinganut, Nâleklavut opigomut, Nuname kilangmelo.

\section{Mel. 166.}

Der, den man durch den Kreuzestod. 146 .

1. Taimna kikkiæktortaumut Tokкolugo pijaujok, InôKojomangimatsuk, Mânna Gûb ômartipâ. Inôtsine tunnijane Ama tigulerivâ; 
Illûnatiglo toккojut umarput tâpsomunga.

2. Erkaumanadlarpoк illa: Naglingnermut tokкоvок, Naglingningat nungutipâ, Aungêrotidlarlugo,

Naglingningat illuvermut Aglât illijautipâ;

Naglingnerub ômanartub Tokкomit tuppaktipâ.

3. Ômartomik oKautsauvut Okpertokotingille; Omartor raujingippatsuk Tungaviginagole, Sâtanib ômisudlartub Pitsartudlarninganut Pijæksaunajalaukpulle Sâlagijaudlarlutik.

4. Jêsusele ômarmingmat,

Inôtsainarivullo;

Tungavigigamitsuglo, Pairijigivlugolo.

Illa tokKungmat kejungme,

Sullinane pingilax,

Inôniarkovlutale

Nungusuitomuttauk.

5. Inôsek pigigaptigo

Jêsub naglingninganut, Ikkinginut anerningat Tessiudlarmattigut; Okpernerlo naglingnerlo Tagva pingortitauvuk;
Ônarsijaulerlutalo,
Ômame opigomut.

156. Mel. 185.

Ave zum Heraustritt aus der Kammer. 148.

1. Salutitaurotit Jêsusiga Illuvernit Kaigavit,

ToKKO sâlagigangne pivlunga,

Saluterkâlaulagit!

Timingne ikkilersimagavit,

Ânanaujotit erkilaulagit;

Kugvinut ungagapkit,

Najutsainalaulagit.

2. Manigoijotit kiksartunnik,

Makkikammertillutit.

Okpertokotitit siarklertut Mannigoromavattit;

Illangat sakkervigekârangne

Assialo illænâkarnermut Salutitaukolerpat; Taimaktauk naglikparma.

\section{5\%. Mel. 132a.}

Als Jesus auferstanden war. ${ }^{*} 206$.

1. Jêsuse makkisimangmat Sabbatime uvlâkut, Marîab tækkoKâlaukpâ ; Jêsus Marîalaukpoк, 
Tagva keviasâdlarpok

Pâmaktervigilugolo

Niplerpor : Nâlegara!

2. Marîab Magdalênable Salutitaudlarninga, Taimaktauk pâmakternin-

\section{ga}

Jêsub ittigganginnut, Tarnima ânanaugivait; Opigomullo ijîngma Kugviorutigivait.

3. Ômamut nakorivara, Kujagidlarlugolo, Inuit Mariatut-itut Tussugitsainarmagit; Ajortokullôvungale, Gûdele nertudlartaule! Perkutigivângatauk.

4. Ma’gdlênaugalloarnanga, Sulle ajortôvunga; Timigalo kemmagupko Assiptut inuktitut, Tagva tautungnerme illa, Mariatut ilârpunga, Nâlegak tækkungmago.

5. Pigigapkit, Jêsusiga! Pimniktauk pillipagit, Timigalo ômattiga Tamarmik pigilaukik; Nuname pijarêruma Illingnulle ailaungminga, Merngoêrserkovlunga.

6. Jêsuse aiparigapko Uvlâmille unnungmut,
Ikkinginut nippipunga Ajulitsainartotut; Aungminullo ômattiga Missuktênalermagole Kuviasuktipânga.

\section{Mel. 10.}

Ach einem Thomas Glücke. 150.

1. Tômasetut tækkuta! Tækkojomadlarpakka, Jêsusiga! ikkitit Aggamnullo raulugit.

2. Nunamêtillungale Okpervigigapkille; Ijîmnut tautungnæglo Kuviagidlarpagit.

3. Ikkitit tækkogupkit, Kuviasulerpunga, Angijovaksoarmik, Taijaujungnangitomik.

4. Timingne ikkit auggit, Tômab aktoramigit, Niplialerpok imâk: Nâlegara! Gûdiga!

5. Ikkitit aitangajut Illagêktokotivit, Erdligitsainarpaillo, Torkortauvigivaillo.

6. Taimaktauk Jêsusiga Tuksiarpagit mânna: 
Mâne inôtillunga

Ikkingnut tork rrtinga.

7. Ikkitit tækkogupkit, Kugvinut ungagilugit Kunnikpakka tâpkoa Opigigapkit illa.

8. Kanoк pivik Gûdiga? Okpertungortilaunga, Tautungigalloarlutit Ômattib najorpâtit.

\section{8, a. Mel. 185.}

Wenn der Herr, der aus dem Grab erwachte. 151.

1. Nâlekab, makkitub illuvermit,

Nellipsalerangagit

Inukotine salutidlugit,

Illa piloringnarmêk!

Taimaktauk uvluptingne tamaine

Nellipsartaukattarta tamâne!

Ullapirsaidlarninga

Missigilavut tagva!

2. Kugviortut paingumut, naglimut Ômartub mannigorpait,
Inukotinelo kiksadlartut Arvertarkattigivait;

Nokкarkojaujomatuinarlune,

Igluptingnut itterkattauvlune

Avguivok kakкôjamık

Pângat inôtiksamik.

3. Nagligivingârvigivâtigut

Kiglisiniarluta ;

Sorlo Tômas okperkovâtigut

Unêt tækkolugata.

Naglingnipta nellâgôrninganut

Kuviasuktilavut Pêtrusetut,

Mallidlarlavut ama

Tômasib illusinga!.

4. Mânna sulle missiginaravit

Ômavlutit, кuјаnaк!

Tarnipta pâmaktervigivâtit

Imâk: „Gûde Nâlegar!“๘ Anernivit ômartitsininganut

Sulle kibligungnadlarpaptigut.

Kait nutângortiluta, Illiktut-erkovluta! 


\section{KRISTUSIB KILALIARNINGANIK IKSIVANING-}

\section{ANIGLO GÛDIB TALLERPIANE.}

158 Mel. 124.

Welche Grottesgegenwart. 152.

1. Missiginarmêk! Jêsub ômatiga

Sakkijâlerangago Jêsusiptut.

Atầta, Ernek, Anernek ômatimnut

Najûput Jềsusikut.

2. Uvlullo unnuallo nâlaulakka

Kippijutigilugo Jêsusiga.

Najoromadlarlugo soraernanga

Killanainarlanga!

3. Opigitsainarlago nâlengnartok!

Mannigorvigigapko pinnarnartor;

Inôgutisivlunga tokkunganit, Pivluta tokrungmat.

4. Pivlunga tokrogame illijaujos,

Uvlut pingajuænne maklkilauкрок; Aposteliminullo ômardlune Takkoterattarpor.
5. Pivluta paungardlune кilaliarpoк; Inuktulle-idlune iksivavoK Gûdib tallerpiane Nâlegaujox NertortautsainartoK.

6. Kanō ikpigilaukât illinniartut, Jêsus kilaliarmat ârlungajut?

Kibliktaumariklutit ungagivât Kuviagivâllo.

7. Kanorlo nâlektine pinerpagit?

Paungarkârane sulle saimarlugit

Najorkattaramigit ômarmijub Naglingniktotôjub.

8. Gûde kujagilavut, inungita

Sulle ôgungnarmatsuk saimaninga!

Unêt takkuksaunane katjârtine Najudlarpait sulle.

9. Kakugole Nâlekab akkarvia 
Nelliutijomârkâ?

Nellovara.

Nâmaksituinartale tækkonago

Okpervigivlugo!

\subsection{Mel. 228.}

O Sohn, du Gott von Ewigkeit. 212.

1. Erneк, Gûd issokangitor, Illa piloringnalaukpoк Nelliutok sakkervît!

Ijit tækkojut illingnik Siut tussartor nippingnik Pilorilaukput illa.

Nellautaijut attannîllo

Unnudlartut tussugilaurpât

Nekkorsijutiksak tâmna.

2. Annikitotuinarmigle Sekkerpok kaumajok una Uviningme nuname; Killamik, Nâlegar okarpor,

Tautugungnainiarpose Ijipsingnut kênamnik. Piloridlarpulle tarnit Tækkonatik okpidlartut! Aksârtaujungnangimatta.

3. Makkinime kingorngane; Uvlut 40 gille sulle, Nunamêtualaukpor; Tagva Ernetuak tâmna
Uttilauкрок illa ama Atâtab tallerpianut. Illa, kia kaujivagit Illagijangit tagvungale Pivlutalo âkiktangit?

4. Siumut tussartaungilak Ijimut tækkojaungilak Taijaujungnangilarlo, Ânanaunek âkiktanga Takpânele tâpkonunga Okpertokotiminut. Illa kêtatuinarmik Allagaigigupko, tagva Ômamut tussugivara.

\section{Mel. 146.}

Als unser Josua. *213.

1. Kingorlerpaujomik Nâlegapta Jêsusib, Okpertokotine Âpait Betâniamut; Kingorlerpâmiglo Aggane puttoggit Kollangiutitipait Saimartidlarlugit.

2. Saimartimmagillo Nuvujab neksalerpâ, Kilangmut nunamit Takpaungaudsaudlarlune; Okpertokotingit Tautudlartillugit. Nangertut tagvane Ârlorvigivlugo. 
3. Kingonertugivât, Tækkolugo ungamut; Utterkojomavât Nâlegarigamitsuk. Kugvinut ijingit Aupaluktitauvut Pâmangalerlutik Tuksiaramitsuk.

4. Angutigle magguk Kilangmiuk Engelik, Tagvane nangertuk KakKortamik annorâlîk Imâk okalerpuk: "Illipse angutit "Galilêamiut, "Sôg ârlungavisêe?"

5. „Jêsusib tâpsoma "Mânna kilaliartub "Uvlok kaujimavâ "Tikkitsomârviksane; "Tækkojomârpasse "Kilangmit akKartok, "Ikkiliksôvlune "Sorlo kongmuarmat."

\section{Mel. 184 a.}

Wenn schlägt die angenehme Stunde. 154.

Illanârijama uvlunga AkKaromârviksangale, Kuvianadlartok каKugo Nelliutijomârpâle? Kakugo Kaikojomârpânga
Ijimnut tautulerlugo?

Kakugo erkiniarpigo, Najordlugo pairijiga?

\section{Mel. 185.}

Seine Jünger, welche ihn im Glanze. *216.

1. Illinniartingit tækkonârtut Nuvujab neksarmago, Salutimmatsuglo rilangmiut

Tikkilermat takpaunga;

Kiksarnermiglo Kuviasumik

Illumingne ikpigidlalaukput,

Illannârijitigle

Tautugungnairamitsuk.

2. Kiksartorsôlauktut okpernarput!

Mannigudlarlugillo,

Engelik senninginne nangertuk,

Imâk orautingmagit:

„Jêsub tâpsoma tækkolauktapse

"Takpânget tikkitsomârivâse."

Killamik akкariak

Okperigivât taimak.

163. Mel. $166 \mathrm{~b}$.

O Du dort von Bethania. 156.

Jêsuse Betâniamit

Kilalialeravit, 
Illatille kemmaklugit;

IllagêktoKotivit,

Nâlegak, tækkonârpâtit,

Kongmuartitaugavit,

Ama tikkerkogamitit

Killanâridlarpâtit.

*2. Kilangmêkalloartotit

Gûdib tallerpiane

Kattangutigivaptigit,

Puigungilaptigut;

Ômatiptalo ômatît

Missigekattigivât

Tamainik oKumaitunik

Kuvianartuniglo.

*3. SâlaKarviksioravit

Uvlome, pâmakpogut

Ivjomit illa pijogut

Sângne ivjornaitotit!

Kilangmelo nunamelo

Attaniodlaravit,

Uvaguttauk angerpogut

Nâlainaromavlutit.

*4. Illuptingnêtut tamaita

Illingnut torângitut,

Tamaita nunamit pijut

Asserudlalaukittôk!

Apkutiksarsiorluta

Nunakullungmit paunga

Kilaliartingmitigut

Nâpkiningnersoarnut!

\section{Mel. 132 a.}

Auf Christi Himmelfahrt allein. 155 .

Jêsus kilaliarlaungmat

Kilaliarivunga,
Kiksarnartullo tamaita Sâlaginiarpakka ;

Niakorle kilangmêmat Avatine illa Jêsub Amuarniarivait.

165. Mel. 146.

Ich fahr' auf, spricht Christ der Herr. $* 219$.

1. Okpertunut imâk, Nâlegavut okarpok: „Kilaliarpunga "Atâtamnut Gûdimnut, „Atâtapsingnullo "Gûdipsingnullotauk "Tâpsomunga mânna „Ullapkoserapse.“

2. „Apkutaurungalo "Miksexârtôvlungalo "Inôtetôvunga, "Napârtotôvlungalo "Waineliksauvlunga, "Akkerôvosele: „Uvapkut kissiane "Atâtamut pivose."

3. "Atâtab nangminer "Illa nagligivâse, „Nâlegauvingminut "Tâpsoma Kaikovâse : "Naglingningane-gôK "Itsainaleritse, "Okautsimniglotauk "Mallitsainarluse." 
4. „Naglikapse illa, "Najortigilaurapse, "Taimak sorairnanga „Nagligênarivapse; "Naglingnimnêkupse „Illupsingnênama, "Sumik Kennugupse "Tunnijomavapse."

5. Atâtamnut paunga „Ailârpungale mânna, „Anernek mikselik „Innimnut tillivara „,llipsingnut, ôma "Hailigeovlune "Miksekârtunulle "Tessiolârpâse."

6. „Nâlegak Kristuse ! „Nakorênarlaptigit. „Naglikaptigulle "Pivluta toknogavit. "Tarniptigut mânna "Illingnêlaungmita "Nunamêtilluta, "Angergaruptalo."

\section{Mel. 22 b.}

Nun freut euch Gottes Kinder all'. 158.

1. Gûdemut kittorngaujose Mânna kuviasugitse! Nâlegak kilaliartok Sorairase nertorsiuk.
2. Nâlegar pijarêrtitsivor, Inniksamik uvaptingnut, Pênartaptingnik takpâne; Nertorsiuk sorairase.

3. Piulijauvogut illa Gûdib inûblo Erningat Sâtan tokkolo ajortut Nukingêrotilaungmagit.

\section{6\%. Mel. 54.}

Wir danken dir Herr Jesu Christ. 159.

1. Jêsus kujagivaptigit Kilangmut kongmuaravit Gûde nekkorsitilaukit Timivullo tarnivullo. Hallêluja!

2. Ama tækkolârpaptigit Kilangmit akkaleruvit, Avativit mallidalârpâtit Niakorênaramitit. Hallêluja!

3. Kilangme serrijomârput Issokangitoksoarmut; Tamaine najoramitit, Sorairatik erkarpâtit. Hallêluja!

4. Nerkigivavut okautsitit; Katjartivit missigivâtit Kaumanernut torârpâtit 
Kênallo tækkonâlerpât. Hallêluja!

5. Kajungervigivaptigit,
Kissivit tæt:igællutit, Mannigorvigivaptigit, Pitjutekarapta aungnik. Hallêluja!

\section{GÛDDEMIK， SAKKIJÂRNINGANIGLO PINGORTITSI-}

\section{NERME, AULATSININGANIGLO.}

\section{Mel. 230.}

Auf, auf mein Geist. 165.

1. Opallungailaurit tarnîk, Gûd, Nâlekat nertudlarlugo!

Attê, attê tuppalaurit!

Illukut kippivigiuk

Gûd ômattib mannigorutâ, Nertornartotôngmat illa:

Tamanna pivlugo

Opigidlaruktôk,

Kujalimut,

Ungamullo

Itterillo

Ivsornaitovekotinganut.

2. Sivorliovor tamainit, Kingorliojomârivoktauk, Illûnâne missiginarpok; Tâpsomane peкangilak Tâktomik, Kaumaniudlarmat;

Pitsartunek ômanêtor Sangêniængilak, Sokroseranelo, Kanoêtor
Kaujingilâ,

Taimaitsainarpor

Issokangitoksoarmut.

3. Piloriktorsôvor illa Illitarksijok tâpsominga, Atâtagârungnartorlo; Perkutaunerartaungmijok Tâpsomunga, Kaujijaujorlo

Ernetut panetullônêt

Nâmaktomik sulle

Tækkungilarale,

Kaujivungale

Nelliumik,

Tautugvimnik,

Kaumajome kênanganik.

\section{Mel. 218.}

Wie gut ist's doch in Gottes Armen. 169.

1. Nekkoksinar nutakкatitut

Gûdib agganginêkupta, Naglingningat kiblikpattigut, 
Sivorajuitsainarupta.

Tarnîgle siarklerungnaerit

Gûdivut soKkositsangimat,

Kaut tamât inôtsainarmat;

Gûd nellâgôrpor naglinerme

Aungminik kovisilaurame

Ômattivit najorliuk.

2. Sinnaungajungnailutit

\section{mânna}

Gûb sângane merngoêrit, Aksârtauniarnak ôminga, Pivianarnerpângongmat;

Gûb Kristusekut anneringmattit

Tâpsomalo tessiormattit, Serngnigitsainarmattillo. Âniaguvit Kaujivâtit, Nâmaktomik tessiorpâtit, Puigutsængilâtillo.

\section{Mel. 11a.}

Keine Schönheit hat die Welt. 166.

1. Sillab pinnarnarninga

Tækkogupko, Jêsuse.

Pinnarnartunnit tamainit

Pinnariluarpagit.

2. Uvlâkut kaulerangat Sekrinerlo nuingmat; Tautuit pinnarnartok Erkaumalerivara.
3. Takkerlo tækkogapko,

Kilaub uvloriangit, Pingortitingat illa Angijok erkarpara.

\section{Mel. 11 a.}

Himmel, Erde, Luft und Meer. 167.

1. Kilak nuna immarlo

Nertordlerput illingnik, Tarnimatauk Jêsuse Nertutsainarlitille.

2. Gûde mallungnartomik Tarnima missigivâtit: Kinaunernik kinaunimnik Erkaititsainarminga.

\section{Mel. $151 \mathrm{i}$.}

Gross ist der Herr und mächtig. *234.

Nâlegak angijôvor,

Piniarningillo Angijovaksôgivut; Kamagiguptigik, Kuviagidlarpavut; Illisimanermut Tamaita illingmagit, Pairitsainarlugit.

172a. Mel. 595 a.

1. Idluarsaivotit Nâmamariktomik 
Sillaksoarme, Gûd igvit, Tattanınadlartomik.

2. Pijautitsivotit

Pidlaræksaujunik, Kagvartitsilutit sanningmit

Nikanarsârtunik.

3. Nâletsiartomik

Ômatekartinga!

Pilanga pijomajarnik

Kuviasuklunga!

\section{Mel. 159.}

1. Illuserilauktanginut

Inuit nuname

Ajokertorivaptigut

Uvaguttauk Gûde.

Itsarnitak, mânnanitak

Attutsiarpat, Nâlegak,

Pigannertitsomavluta

Takpaungartilluta.

2. Gûd! inuit kinauningit

Kaujitsiarpattit!

Kinaungmangâttalo, igvit Adsigêktipattit.

Illûnatik illusingat

Idluartôkotuinarpat;

Taimaimattauk mânêtogut

Âxisulauktigut!

3. Tessiutsainadlarangne Inugasæksuit
Siamangajut nuname,

Tattamnadlarpotit.

Uvangatauk tessiunga, Mallitsiaromavunga

Sunatuinaujutigut

Takpaunga rilangmut.

\section{2 e. Mel. 7.}

1. Pitsiarnermullo

Pidlarningnermullo

Inuit ômatingit

Sullivigênarpattit.

2. Taimaktauk uvanga

Missigênarlanga, Aggakpît saimanartut Amungmanga kilangmut!

\section{2\% d. Mel. 8.}

1. Tattaminiarpunga

Nâlegak Gûdiga, Issumaksarsiorpat Ômatikulluga.

2. Piniarnersoatit Nellonakôdlarput, Idluarkutiksamulle Illingatuinarput.

3. Sorlo pingortigangne, Gûde, nunamêtut, Illikkut mânna sulle InôseKainarput. 


\section{pingortitsinerme, aulatsininganiglo.}

4. Issumamingnik inuit Piniarkôrunik, Sappidlarput illingnik Kivgartutsaratik.

5. Kunnungipattalônêt, Kunnukpattalônêt, Tikkiutititauvok, Gûde, nâlegauvît.

6. KanoKtôK illûnatik Nippît nâleklitsuk! Igvillotaur ômatîk Kamagitsiaruk!

\section{2 e. Mel. 90.}

1. Nuname pijokartune Nâlegak angijôvor, Okitune, okumaiture Tamaine sullidlarpok; Nippingat uerisarlugit Kaikotsainarpait inuit.

2. Kaumajok Gûdib sennavâ TârtoKarkojivorlo, Ullapirsaullo tunnivâ Pijæksaukôngitorlo ; Taimak adsigêngitokut Sennavigênarpâtigut.

3. Gû̉b pitsiarninga kilangmut

'Tôrpok, illûnânêpor, Miksekârninga nuvujat Ainingit tikkidlugit; (Img. 36, 6.)
Kakkialernermut illa Tessiorpoк pitsiarninga.

4. Kammajomangitokarpat Gûb piniarninginik, Tagva illungertorningat Pidlarpâ angijomik; Illa kammajomangitut Illiorpait ikkomatut.

5. Gûmut sennaviogupta, Kammatsiadlalaukta, Ama, nippungalaurupta, Uttersarailaungmita! Saimanartungorkovlugit Aglât Gûb pidlarningningit.

\section{2 f. Mel. 159.}

1. Sunaungitôlungilatit Gûdingoatitut, Aulatsijôtsiarpotit Ômamariktotut. Tautukpogut inôngitune Inôjunelo nuname Aggakpît sullininginik Tattamnadlartomik.

2. Kivgavît aggangitigut Sullidlalaukpotit, Inôtsib illinganingagut Aulatsivakpotit; Attanionît nuname Sakкijârpor soraerane. Taimaidlarmat, ômajotit, Tættigivaptigit. 
182g. Mel. $585 \mathrm{~b}$.

1. Gûmik ijersimajomik Taijaugalloarpotit Tukkisijaukattangimatta, Gûde, piniarnitit;

Kingorngane Sukutsiane Tâlutjartaularpotit.

2. Illa nellonadlalaurtut Sakkijâtsialârput Ullapirsautiksainaujut Inuit ômatinginut. Opinarmêk ! Kujanarmêk Gûde tessiortivut!

\section{In. Mel. 1321.}

1. Inungnik sannituinarnik Sivôrataililanga! Kiksanaralloartomik KanoetoKartippanga. AttauseK sivôranarpoK Gûde aulatsijorsôjoк, Kammagijaksatôjor.

2. Ômigijiptingnullônêt Sivôratitsijunut Illagijaptingnullônêt Uerisaidlartunut Tammartitauniarata! Gûmulle aulataulauxta, Omunga kissianut!
3. Nipperaruvit nuname Nâlegak, tussartinga! Tamaine pijokartune Illingnik kammalanga! Nuname tukkisijapkut Torârtilaunga kilangmut Nâletsainartôvlunga!

\section{Mel. 45.}

Warum betrübst du dich mein Herz. 170.

1. Ômattiga!

Sôg kiksarkît

Nunab pingit pivlugit? Gûde trettigilauruk, Ikkajortetôjor.

2. Ajoktarnik

Nellungilar,

Allaromangilâtit,

Piuliniarpâtit

Kappiagijarnit.

3. Atâtaga

Kittorngarnik

Kemainiangilattit, Tættekartut illingnik, Sutairniængilat.

4. Ikpeksarlo

Uvlomelo

Issokangitomullo

Tâmnautsainaraville

Tættigidlarpagit.

5. Ômattiga

Ajoksartor 
pingortitsinerme, aulatsininganiglo.

Piksakartilaungmiuk Tagva nâmaksivunga IssoKangitomut.

\section{Mel. 106.}

Wer nur den lieben Gott lässt walten. 172 .

1. Gûdemik tættetuakartor Pijangit nâmagivlugit, Mannigortiksatuakarpok, Kiksarnarpallônêt suna, Gûdevingmik tættekartor Tamât ikkajortaulerpor.

2. Serrinarvît nelliuningit, Gûdib kaujivait kissime; Ikkajorluta pivâtigut Mallitsainaruptigole;

Ajungivigidlarluta

Opingarpâtigut ôma.

3. Kennuêlune uttakkijok Gûdib pijomajanganik; Tâmna tukkisitsainalerpor

Ajungitullininganik, Gûdib anneringmattigut, Ajoksarkongilâtigut.

4. Gûb apkutâ attutsainaruk, Nellâgôrasuarlutit;

Tagva saimartaujomârpotit

Tættigigungne tamainit: Gûdemik tættekadlartor Kemmaktauniatsængimat.
175. Mel. 79.

In allen meinen Thaten. 174.

1. Tamât piniarnimne

Ikkajortaukovlunga

Gûd kennuvigivara,

Piniarkovlungale

Idluarijanganut

Gûdib pijungnartimmanga.

2. Pinasualernera

Issumajârneralo

Sulliniængilâk ;

Tamêt. illinganera

Perkojæksarivara

Tâpsomale agganginut.

3. Taimaimat tarnîk mânna Gûd kissiat tættigiuk, Pingortitigijat;

Piksoma Atâtavit, Tamât sapputingmattit Sumullônêt pijaukonnak.

\section{Mel. $151 \mathrm{~g}$.}

Befiehl du deine Wege. 176.

1. Inôniarnernigle

Pijomajarniglo

Gûmut pititsilerit Sillab imarbiublo, Annorib nuvujallo Aulatitingænnut; Ôma apkotiksatit Âkitsungnarmagit. 
2. Kuviasukovlutit Gûde tættigiuk, Piniarnerlukonnak

Nâletsainarmiuk, Nangminer pijomamut Issumajârnægle; Tamaine naglingnermut Pairitsainarmattit.

3. Illûnâne Gûdiga Apkutekaravit, Piniarnitit tamaita Saimautigivavut; Agviarlutit Gûde, Nellipta ajorpâ, Kittorngatit nuname Nâpkigidlarangne.

4. Satanit illûnatik Agviaromajut, Gûb piniarninginik, Sapperput tamainik.
Gûdib pijomajane,

Perkojanelotauk, Nâmaksititsainarpait, Nellautserkovlugit.

5. Tarnîk! nerriulaurit Innertuinaglo, Gûb piulijomavâtit Kiksautigijarnit; Ôma nelliuninga Uttakkilerugle Saimautsomangmattille, Serritilutillo.

6. Gûdib piniarninga Tarnîk nâmagiuk, Tattamnartulliningit Pivlugit, opigiuk. Atâtatut naglimut Issumagivâtit Pêrpaillo aktornartut Agviarkonnagit.

\section{GÛDEMIK PINGASÔLIJOMIK.}

17\%. Mel. 185.

Atâtagigapkit nutakkatut Êgangarvigilagit!

Kattangutiga nâpkigilaunga

Manigulaungmingalo.

AnerneK anânatun-ilutit

Pêjakit kiksarnikka tamaita. Gûd pingasôlijotit Sapputsauvigivagit.
178. Mel. $132 \mathrm{a}$

Allein Gott in der Höh' sei Ehr'. 181.

1. Gûde nakoridlarlavut Kujagênarlugolo, Nâpkigitsainarmattigut Pairiluarlutalo, Gûb Kuviagivấtigut Ullapкutekalerapta Jêsub ânianganut. 
2. Atâta! nertorpaptigit, Pâmangavigivlutit, Nakorênadlarlutillo Nâlegauvît pivlugo. Ajugakangitôgavit, Perkojatit malliktaulit, Nâlegaujotôgavit.

3. Jêsuse Gûdib Erninga, Inungnik illûnainik Ullapirsaijotôgavit, Nuname tammartunik. Nâlegak, Gûb saugârsunga

Tuksiarpaptigit mânna, Nâpkigilaungmittigut.

4. Anernerlo ajunginek! Mannigortetuavut, Sâtanib uivêninganit Sapputilaungmittigut, Kristub piulingmattigut Aungminullo tokkominut, Ajortunit tamainit.

\section{Mel. 185.}

0 Vater, der du den Heiland. *250.

1. Atâta! tilliklilauktotille Ernernik uvaptingnut, Aksârtautailititigut mâne Piuliklerninganik.

Ikkajortigut inôkovluta Tâpsomane, Kuviagijarnut, Kittorngautsainarluta Illingnut Gûd Atâta.
2. Jêsus Kristuse Gûdib Erninga

Tillijaudlalauktotit,

Kilangmit uvaptingnut tamaunga,

Piuliklerkovlutit

Inungnik, assiomadlartunik :

Kailaurittôktauk ômattiptingnut

Innigênarlugillo

Nunamêlertilluta.

3. Anernek Gûdiojotit illa!

Mannigotsainartigut

Kappiagijaptingne tamaine,

Tukkisivaktaptingne:

Inûlidlarlugillo tarnivut, Nekkokserkovluta illuptigut

Ungamarerkovluta

Jêsub ajokertûtânut.

\section{Mel. 218.}

Gott der Vater wohn' uns bei. *251.

Gûd pingasôlijotit igvit Innigitsainartiguttôr, Asserorkoniaratigut

Ajorungnaitilutale; Sapputitigut Sâtanemit; Okpernermêtsainartittigut Tungavigimariklutit; 
Taimak kemâtittigut nellâgut

Ikligunernik mamaitunik, Sâlakarmarekovluta.

\section{Mel. 132a.}

Was Gott, der Vater und der Geist. 256 .

1. Gûb, Atâtab Anernerublo, Ajungitulliningit, Saimaningillo inungnut, Jêsus pivlugo pivut: Kennuguptalo Gûdemit Jêsub attingane sumik; Ahailârviovogut.

2. Kailaurit Jêsub akrane Pituaridlartapta, Uvaptingnênarlutillo Gûd pingasôlijotit! Saimanartovingme mâne Okperijomavaptigit Inôvigivlutillo.

\section{Mel. 228.}

Halleluja, Lob, Preis und Ehr'. 184.

1. Hallêluja, nâleklugo Nertoriartorlavulle, Gûde pingortitsijor IssokangitoKsoarmut! Kutsaservigidlarlavut Avagosuktotôjok; Attê, Gûde Nâlegaujor Ivsornaitor
Opigilavut, Ômamut kujagilavut.

2. Hallêluja! nakudlartub Gûb Erningat tokкotaujub Annerilaungmattigut; Aungmut pissiaringmattigut

Serparsarlugit tarnivut, Kittorngaringmattigut; Tagva illa inôviksak, Piloringnek, Pigijavut, Illagêkapta aunganut.

3. Hallêluja, Gûd Anernek! Mânna soraijuitomik Nertudlarlaptigille Erniangotinnaptigut Pitiluta Jêsusemut Anneridlartiptingnut; Illa tagva kuvianak, Piloringnak, Tarniptingnut, Piksarivavut nellâgut.

4. Hallêluja, nâleklugo Gûdivut, nertorlavulle, Attingalo ômamut! Kilangmiut illagilugit Imgertatôk nertornaumik, Kuviagilavullo:

Hailig, Hailig, Hailig Gùde,

Nâlegavut

Gûd Zebaot

Nakoritsainadlarlavut. 
NÂLEGAPTA JÊSUSIB KRISTUSIB ATÂTANGANIK ATÂTAGIJAPTINGNIKTAUK.

183. Mel. 82.

Lieber Vater, unser Herz. 263.

Nagliktor Atâtavut!

Kujagidlarpaptigit,

Ernerpit ânianganik,

Aunârmatta avatingit.

Piulijomavluta,

Tоккungmallo pivluta.

\section{Mel. 10.}

Lasst uns mit süssen Weisen. 188.

1. Gûde nâpkigosuktor Atâta saimarsaijok Kujagivigilavut

Nertorlugo ômamut.

2. Gûdib ajortônerpât Kittorngarijomavait, Piulijaukolaitalo Tamaita, Ernermigut.

3. Atâtab naglingninga Missigileruptigo, Tagva tættekarnermut Ârlorvigênarpavut.

4. Illale ajortavut Tamât tuksiarnermut Taiguptigik Jêsumut, Kippilungilâtigut.

5. Nertornautiksarnulle Pigannertilauktigut,
Tunnitsivigiluta

Tarnipta piksanginik.

185. Mel. 214.

Sollt' ich meinem Gott nicht. 189.

1. Tuksiartuksaungilanga,

Gûd kujagênarlugo! Aukak, illa naglingmanga, Ikpigitsainarapko.

Naglingnermik kissiane Ômattinga tættæpor.

Piniatsainarporlo

Pairilugit kittorngane Gûb nagligosungninga Nungusuipor illa.

2. Ernine ivlerinago Gûb tunnivâ pivlunga, Aunganut piulivlunga Pitlartaujutiksamnit Nellâgulle saimaninga Ipaksinatsængitor, Uvamnut innornarpor, Kujalijutigivara; Gûb nagligivâtigut Issokatsængitomut.

3. Tarnima pilloreutiksâ Issumagiluarpâ, Ânianingat timiga Pairilualerivấ, Sangênermut sungikuma Piniaromanimne, 
Pitsartunerminulle

Ikkajoriartorpânga ;

Gûb nagligivâtigut

Issokatsængitomut.

4. Sinnikuma pairivânga

Nekkorsitilungalo,

Kaut tamâllo naglingninga

Nâpkigosungningalo

Tukkisivakka. Gûdima

Tessiulaungippanga

Kappiasudlarnikka

Pêjartaunajatsængilat.

Gûdima naglingninga

Nungusuipor illa.

5. Gûdib naglingninga taimak

Nungusuitotôngmat,

Tuksiarpagit Atậta,

Tunnitsiviolanga;

Pitsartunimnut tamainut

Erkitsomadlarapkit,

Uvlullo unnuallo

Inôjungnaerkârtinnanga.

Pâne ungagilutit

Nakorênalârpagit.

\section{Mel, $\theta 6$.}

O Gott Vater im Himmelreich. *270.

1. Atâtak. kilangmêtotit Kennuvigidlarpaptigit, Attît nakoridlartaule, Nâlegauvîllo kailaule Sorairata perkojarnik Mallingnitsainarkovluta.
2. Piksakartitsainartigut,

Issumagijungnaervigitigut

Sorlo pivigigaptigik

Idluitullivigijivut,

Tamapta serngnigitigut

Perkonnata ajortomut.

\section{8\%. Mel. 58.}

Also hat Gott die Welt geliebt. 186.

1. Gûd nagligigangne inuit

Tunnivat tâpkonunga Ernit

Piulijaukovlugit âniangagut,

Inôkovlugillo toKKungagut

Aungagullo.

2. Ernît tunnigangne pivluta Kanok Kujagijungnarpagît?

Sillaksoarmiut nachonadlartut

Kristusekut anneridlarangne,

Tattamnarmêk!

3. Angijomik kangusukpogut Issumaksarsiolerupta,

Kamanginiptingnik, âkiksortarnik,

Ernerpit piuliklerninganik Uvaptingnik. 
188. Mel. 22.

Unser lieber Vater, du bist. 187.

Tættigitsainarpaptigit,

Ernivit Jêsub Kristusib

Kattangutigingmattigut

Atâtagigivaptigit.

\section{Mel. 125.}

Atâtagigaptigit

Gûd nertorpaptigit!

Pattangaitigaptigut

Mannigorlutalo

Tamât kiksarniptingne,

Tessioraptigullo

Sâvigivaptigit.

\section{Mel. 9 .}

Der $\mathrm{Du}$ in dem Himmel bist. 190.

1. Kilangmêtotille Gûd!

Jêsus Ernetuat

Kattangutigigaptigo

Atâtagivaptigit.

2. Attît nakorijaule!

Kaujileraptigo ;

Nellonarajalauktor

Jêsub taingipago.

3. Nâlegaunît kailaule!

Ernerit tamainut

Naipertortaukovlugo,

Tokkotigingmagit.
4. Sorlolo kilangmiut

Perkojat mallingmatsuk

Mânetaur malliktaule

Okpertokotingnut.

5. Uvlome piksaptingnik Tunnitsivigittigut, Pitsartutilutalo Jêsub tokkunganut.

6. Issumagijungnaikit Ajornivut, sorlo Pijomangmigaptigik Inukattivulle.

7. Jêsusib Nâlegapta Kennuêdlarninga, Kammagidlaraptigo, Inôtigivavut.

8. Ôktortaulungnartomut Pititaukonnata, Tættigênarpaptigit, Sangêtoksôgapta.

9. Tamainit ajortunit Piulingmittigut Pijarêrtilutalo Illingnut kilangmut.

10. Amen Abba Jehôva. Attît nertortaule Nakorivaptigille Issokangitomut. 
GÛDIB ERNINGANIK, NÂLEGAPTINGNIK PIULI-

\section{JIPTINGNIGLO.}

\section{Mel. 172.}

Ach Gott, was hat für Herrlichkeit. 191.

Jêsus ânanaujồvlune Nâlengnadlartoksôvok, Kuvianartovingmine Tamainit angnerôjok!

Kemmergovât Engelit inuillo,

Taiksaungitomik serridlarput,

Piloridlartut piulimajulle Tâpsomangat piksarsiortut.

\section{Mel. 132.}

Der Herr ist mein getreuer Hirt. *277.

1. Nâlegar pairijigivara Ôma sapputivânga, Taimaimat, ajungitomik Ajoksatsængilanga; Pairijima nerritipânga Nuname inôtillunga OKautsiminut, illa.

2. Nâlekab nâpkiningningat Inûluartipânga; Iglunganêtsainarama Kuviasudlarpunga, Nuname illagêktune;
Kaikoppangalo kilangmut Najutsainarparale.

3. Piulijiga najunga Aungnullo ikkingnullo Nertoromavagit tagva Opigidlarlutillo; Tarnima ungagivâtit, Missigijungnairunitit KeavoK Marîatut.

\section{Mel. 125.}

Du Schöpfer aller Dinge. $* 278$.

Gûb Erninga! tamainik Pingortitsijotit, Sillaksûb issuanut Nâlegaujôvotit; Sâtikit ômattivut Ajortunit illingnut; Tammartailititigut.

\section{Mel. 16.}

Mensch, du ein'ger Mensch. 194.

1. Saimarsaijotit Jêsuse! Inungorsimagavit, Attitit hailigeojut Mannigortigivavut.

2. Inûliklerte tarninik Âriksulaungmittigut! 


\section{Ômattivullo illingnik Illumertilaungmigit.}

3. Illanâk kennuniptingnut Siutikik mannikik, Tuksiarutittigullo Atâtavit sângane.

4. Pingortitarnik tamainik Aulatsijotôjotit, Kattangutigivaptigit, Puigunginaptigut.

5. Sapputsauvigigaptigit Serngnigitsainartigut, Innôgutigigaptigit Ômartilaungmittigut.

6. Illissimajotôjotit! Tammaleraiguptale Uttertinasuartigut Sâlagijaukonnata.

7. Aggangnut tessiortigut Nutakkatitut illa, Epкêjaivigilutalo Idluaringitarnik.

8. Ômattivut erKartokit Inôtilluta mâne; Illænâridlartigulle Issokatsængitome.

195. Mel. 68.

Seelen-Bräutigam. 197.

1. Gûb saugârsunga Piulijiga,
Ômamut kujagivagit Amugamga ajornimnit, Jêsuse, aungnut, Nagligosumut.

2. Taimak Gûdiga, Nekkoksivarma, Illûnarma, naglingnern ut Erkigamga illænârmut; Serrilerpunga Nagligigamga.

3. Inuk Gûdelo, Gûdib Erninga Inûlerpotit nuname, Piulivlugit tammartut, Aungnut Jêsuse, Inuglo Gû̉de.

4. Ullapkosikit

Nagligijatit

InuKotitit, uvagut

Illingnik illitarksijut

Nagligivlutit,

Ullapirsakit.

5. Kugvioruma

Mannigorminga;

Pivlunga kugvioravit

Kugvikka allarterlugit,

Kugviornera

Pijungnailugo.

6. Serringmiguma, Igvit pigamga, Serrikattigivarmalo, Pâne perkârtinnangalo 
Nâksaungitomut Pilunga sângnut.

196. Mel. 132 a.

Mein. Herzens-Jesu, meine Lust. 198.

1. Nagliktara Jêsusiga,

Kuviagijatuara!

Ômattingnut ômattiga

Ungamut illingavoK;

Ômamut nakorivagit,

Uvamnullo nalingnivit

Nekkorsitsainarmanga.

2. Inôtigigapkit illa,

Pitsartutilaungminga,

Akiklugolo illuga,

IllingalerKovlunga

Ajungitunut tamainut;

Omattipkut, anernernut

Tessiortaukovlunga.

3. Taimak illa pairigumga.

Inôsimne illûnâne,

Tagva kittorngarigamga

Nunamêtillungale;

Kilangmullo najorlutit

Nungusuitorsoarmut

Itterkojomârparma.
4. Illanâtuarivagit,

Illumnik kiblêjotit;

Anânatut pairivarma,

Inûlidlarlungalo

Kanoênimnit tamainit;

Igvit nekkoKsitiparma

Sangêdlarnimne tamât.

5. Pitsartutigivagillo

Sappûlutarivlutit

Mannigortigilarpillo

Kiksarnimne tamaine;

Umiaktorumalônêt

Akkunaksoakarningane

Sapputsauvigivagit.

\section{9\%. Mel. 22.}

$\mathrm{Du}$ bist nicht mehr in dieser Welt. *289.

Mattomanêtsungnaipotit Mânele erkovaptigut, Ajortunille tamainit Sapputitsainadlartigut.

ANERNERMIK AJUNGINERMIK, TÂPSOMALO TUNNERGUTINGANIK PINIARNINGANIGLO.

198. Mel. 203.

Komm heiliger Geist, Herre Gott. 200.

1. Nâlegak Gûde, Anernek, Okpertulle ômattingit
Missigitikit saimanernik, Ikkitikit naglingnernut! Nâlegarle, raumajokut Okpertilaukit Jêsumut; Annerijatille inungnit, 
Inukotivit nertorpâtit. Hallêluja. :,:

2. Kaumajorle okausernut Kaumaksakit ômattivut Gûmik illitarkseкovluta, Atâtamiglo taivlugo. Nâlegak! serngnigitigut Ajokertortaulungnermit, Jềsus okpervigênarlugo Omamut tæattigilugolo. Hallêluja. :,:

3. Mannigoijôgavillo, Illuvut ônartilugit, Nellâgôrtittigut illingnut Illânele kiksartogut. Nâlegarle! sangêtogut. Illa pitsartutittigut Ajortunut ajugauluta Tokкomut sâlakarkovluta. Hallêluja. :,:

\section{Mel. 58.}

Nun bitten wir den heiligen Greist. 203.

1. Gûb Anerninga kennuvavut,

Tunnitsiviojomavluta

Mânna okpernermil

Miksekârtomik ;

Ajokertortaujomavlutalo Jêsusemut.
2. Ajoksarnartovingmênapta Sapputitsainalaungmittigut;

Kaumartilugillo

Omattivulle;

Mannigoijuksatôgavillo

Kiksarupta.

3. Naglingniojotit ômattivut Naglingnernut illumertikit

NagliktigêKovluta.

Idluarijarnut

Issumakattigêktittigullo

Illuptigut.

4. Kaumartitsijox! Kaumartigut,

Illitarksilerkovlutale

Jêsus Kristusemik,

Piulijiptingnik,

Tâpsomungalo okperkovluta

Nellâgulle.

\section{Mel. 89.}

Komm, o komm du Geist des

Lobens. 206.

1. Anernek ômartitsijor,

Issuanit Gûdivut!

Pitsartunerniglo illa

Illumertimitigut,

Târtôjut ômativut

Tagva Kaumaksartaurut. 
2. Kittorngauniptingnik Gûmut,

Missigititittiguttôr!

Mattomunga okpernivut

Nekkorsijaukovlugo;

Kanoêtitaugupta

Annusingorkonnata.

3. Anernek piniarnitit

Sakrertitsainakittôk

Illuptingne; Sâtanasib

Pinasuarpattigut,

Ajugakanginernut

Sâlakartimittigut.

4. Tokкoviksavut pilerpat, Kaujititsainartigut

PiksaKarniptingnik pâne; Gûd tunningmat Jesukut Ânanâvaksoarmik Taijaujungnangitomik.

\section{Mel. 58.}

Du der du Jesu Volk nie vergisst. 213.

1. Piulijipta Anerninga!

Puigunginangne tamaita

Jêsusib inungit

Nertorpaptigit;

Pairigangne tamaita tarnivut

Timivullo.

2. Sillainivut ikpigivavut, Taimaktaur pitsakidlarnivut,
NukkeKanginapta,

Kaujimavogut;

Pitsartutikuptigut kissivit Sullivogut.

3. Taimaimalle tunnidlarpavut

Tarnivut âriktaujomamut, Illa Atâtapta

Ernerublotauk

Anernerublo ajunginerub Pairksininginnut.

\section{0\%. Mel. 4.}

Sei ewig gepreist. 215.

1. Gub Anerninga,

Nertorpaptigit

Tunnigaptigut

Inôniarnermik Jêsusiptingnut,

2. KaujerKârnago

Ômattiptingne

Kaumaupaptigut

Illitarilugo Piulijivut.

3. Kannitanganut

Âlauraptigut,

TautuKôrpavut

Sorlo sâptingne pivluta. tоккојок.

4. Ômattivullo

Ânianganut

Kiblidlartauvut 
Naglingninganullo aumadlalerput.

5. Opigosumut

Kujagivlutit;

Tuksiarluta:

Illuvut âkikit nakorilugo.

6. Nâlegaptingnut

Âniajomut

InôKovluta

Omativut nâlektikit

ômunga.

7. Tâpsomungalo

Okperkovluta,

Soraijunata

Kujagivlugolo opigivlugo.

\section{0อ̊ Mel. 4.}

1. Gûb Anerninga!

Naglimut mânna

Najudlartigut!

Jêsub ikkinginik Kaujitittigut.

2. Tessiortigut

Nagligosumut

Jêsusiptingnut

Tâpsominga erkaititsainartigut.

3. Nippît illume Tussarlavulle, Tamaita uvlut
Killanârkovluta ajungitomut.

\section{Mel. 11 a.}

1. Gûd kujagivaptigit,

Tunnigangne Anernît

Illuptingnut, ômunga

Tessiortaukovluta.

2. Gûd Anernek ômamut

Tuksiadlarpaptigit,

Jêsusib ikkinginik

Nellojungnaitittigut.

\section{Mel. 79.}

Geist Gottes, Dein Bemühen. 216.

1. Gûdible Anerninga

Pairksinît uvaptingnik,

Pinasuarnîllo

OKautaujungnangilak,

KujalijoKangilak

Nâmaktomigle illingnik.

2. Tagva opigosumut

Sângne pâmangavogut

Naglingnît pivlugo;

Kujagidlarpaptigit

Nâpkigijæksaunata

Ungagitsainarlaptigit.

3. Tamaptale nippingnik

Nâletsiarkovluta

ErKaitittiguttôK;

Jêsusivut pivluta 
Kuviasukovlugo, Sujungaitênaraptigut.

4. Gûdible saugârsungat lkkinginik, aunganik, Ânianganiglo

Nellojungnaitittigut Illuptigut aungminut

Óma pissiaringmattigut.

5. Gûdib kittorngangita, Illangat tammalertor Tækkolerungnele
Naglimut innerteruk, Sujungaitilaungmiuk Kakxialersârkovlugo.

\section{Mel. 22 f.}

Gott heilger Geist, du Tröster wert. *315.

Okpertulle nunamêtut, Attausiutsainartikit; Tarnivullo Jêsusemut Tessiutsainalaungmigit.

\section{GÛDIB KAIKOJININGANIK, AJORTU'T SÂGIAR- KOVLUGIT.}

20\%. Mel. 97.

So wahr ich lebe! spricht dein Gott. *316.

Ahamarik! Gûd okarpok, Ajorneliksôjor inuk, Tokкокоngilara illa; Uvamnut sâterkovara; Ajornerille ipperarlugit Uvamne inôtsainarkovlugo.

208. Mel. $132 \mathrm{a}$.

Ihr armen Sünder kommt zuhauf. 217.

1. Ajort ukullôjosetôk Kaisâxitse tamaunga! Angmarsimangmat tamâne Jêsusib ômatinga,
Illûnainut kiksarlutik

Ômamut ajornermingnik Illitarksijunulle.

2. Okartokarpoк : „Ajortut "Illalliorpait illa! Taimaimat kaituinarit, Erkitsomadlarpâtit Piulijomalutillo. Kugviorlutit kailaurit Nâpkiginiarpâtit.

3. Jêsusib kennitsainarpâ Saugar tammarsimajok Nagvâdlarkârnago tâmña: Taimak nagvârtaukogit! Kemmâlaurit ikkinginut, Sulle nelliusimangmat Saimauservik nuname. 
209. Mel. 165.

So wahrhaftig, als ich lebe. 218.

1. „Ahamarik!" Gûd oxarрок,

„Pijomatsængilara

„Ajortullijub tokkunga;

„Kuviagivarale

"Uvamnut sâgiarpat

"Kailugo ômattine;

„Inukotima illangat

"Assiorojomangilara."

2. Jesusivît ômatinga

Kemmergojungnarungne,

Pairksijublo nertornartub

Kennerasuarninga,

Killanârmaridlarmat,

Saukanik tammartunik

Uttertitsijomavlune,

Tagva ungaginajarpat.

3. Gûdîk! nâpkigosungnernik

Tukkisitilaungminga!

Illænâkarnerniglotauk

Oksitilaunga tamât.

Kiksartitsomairapkit

Kajungertilaungminga,

Ajungitamnut tamainut

Ungakovlunga illingnut.

210. Mel. 106.

Wo ist doch so ein Gott zu finden. 220.

1. Nane Gûdekarka illiktut? Taimak kennuêrsârnermut
Ajortolingmik kennertomik

Pêjaivigijomavlugo

Ajorninginik; sâlerpat

Illingnut nâpkoarivat.

2. Nâlegar inuit tamaita

Perkutaungmatta illingnut,

Piulijaukovattittauk mânna

Illûnainik pijomamut.

Sappermattale, kissivit

Sâgiartitsomavattit.

211. Mel. 1.

Gewiss, Du bist noch so voll Menschenliebe. 221.

1. Jêsuse sulle nagligosuktotit

Sorlo taika pivluta tokKogavit!

2. Kejungme, nipleravit, immerungnermik!

Sulle immerungnermik niplerpotit.

3. Illingnik inuit ômisudlartut

Piulijomadlarangne naglimut.

4. Attauserlônêt assioronnago, 
Tokкungnut kibliniaromavattit.

5. Imâk orautsaukovattit kivgarnut:

Ullapirsautekaritse Gûdemut.

6. Ajortunit ajortûnersaugapse,

Piulijiptingnut ainiaritse;

7. Kanoêmangâpselônêt pititse

Jêsusemut, nachogitsængimasse.

8. Idluinerpângomangâpselônêt,

Illipsingnik ômattâ rêmitingmat.

9. Tarnise timiselo sujukpatta

Illipsingnik immerudlarpor sulle.

10. Assiojuksaunerminik каuјiјок

Ingminiglo piulijomangitOK;

11. Tuksiarune Jêsub ittiggangine

Tâmna assioniatsængilarle.

12. Amarkotun-ipallônêt illume
Ablatsângortitauvok nutakkatut.

13. Aputitut niglinarpallônêllo

Ônarsijaulerpor ikkoma sorlo.

14. Ujarkatut tokKungadlarpallônêt

Ômálerpor piloriktitaurlune.

15. Ajornek illume tokкotaulermat,

Jêsub aunganullo ânianganut.

16. Jêsub ittigganginnut pâmagitse

Inôtsine tunningmago pivluse.

17. Jêsub tokkominut $\overline{\text { piu- }}$ lingmattigut

Ittertitaujungn arpogut Kilangmut;

18. Tautuklugo, Kuvianarpor tagva

Piulijaujub kuviasungninga.

\section{Mel. 39.}

Kommt Sünder und blicket dem ewigen Sohne. 222.

1. Ajortose tækkosiuktôk Jêsuse! 
Gûb nâksaungitub Erninga piulijise

Kikkiaktortaujor, niakôsertaujor

Kakkilautinulle, pirluta tokkotak.

2. Kollarnermut okpingitoKaranetôk!

Nutakratitulle illûnata pita!

Sillatulungnerit kikkiæktorlugit,

Piuluatangitut Gû̉b nâpkigingmagit.

3. Passijæksaunerminik nagvârsimajok,

Jêsusib ikkinginut apkuteкаrрок,

Ingminigle ajungitsugijungnaimat,

Jêsub inûlivâ nâpkigilugolo.

4. Illûnatik ajulertut aklujullo

Jêsub tokrotauninga pituarivât;

Ômunga, Gûdemut ullapKosermagit,

Tamanna pivlugo Gûdib nâpkigivait.

5. Ajoksatsainarluta, piniarpogut

Saimaninganut immarbiktun-itomut,
Akluvlutalo ajorsatsainarapta,

Piulije tungagilugo inôta!

\section{Mel. 376 .}

Kommt her zu mir. 223.

Uvamnuttôk kaisâdlaleritse!

Illûnase sænnikterijose, Kiksartullo, nellît kailerpatta,

Ajæktolungilakka Mannigoromavakka.

\section{Mel. 217.}

Mein Heiland nimmt die Sünder an. 224.

1. Jêsusima nâpkigivait

Ajornernik sænnikterijut, Inûblo Engeliblônêt

Ajortangit mannigorlugit, Tarnekut saksârtut aglât, Mamaitut Gûmut ingmingnullo

Perkojanut erkartortaujut

Pijuksat ânianartomut, Tâpkoa kellarudsârpait Jêsusima nâpkigivait.

2. Nagligiluarmattigut Anânamit kittorngaminik, Næglingniub akkartipâ, 
Kilangmit, nunamut pilune:

Ajortuggit inningænne Ómigijaujomavlune.

Inôtsine tunnigamiuk

Pivlugillo tokкo ôklugo.

Gûdemut nâmaksitipait;

Jêsusima nâpkigivait.

3. Mânna angmarmat ômattâ, Nellipsarnartôjoк tarninut,

Ajortunik erksingmatta, Kelluvait ingmingnik passijut,

Idluitullidlarningit

Pêjalerpait, Anernine Tunnivâ ômattinginulle;

Tâpsomalo idluartomut Tessiorpait illûnaita ; Nâpkigivait Jêsusima.

4. Atâtaminut pitipait,

Erkilugit aunârtolingmut Tallerminut, taimaimalle, Atâtab nâpkoarinermut Kittorngautipait ingminut; Piminiktauk tunnijijor Tâpkonunga, inôtsemiglo Nâksaungitomik pingmilugit Angijomik taimak illa! Nâpkigivait Jêsusima.

5. Kanoktôk tækkonâleruk Ômattingat rêmitingninga, Ajortut nâpkigilugit
Kaikolugillo kugviortut, Zoellnerillo; Zachêamut Ittermat, Magdalênalo Kugviormat ittiggangine Kugviorningit pêjarmagit Tainagillo ajorningit, Jêsusima nâpkigivâ.

6. Illa illanâkardlune Petrusetauk tækkosalaukpâ,

Kattadlalauralloarmat! Sorlole taimailiorlune Nuname arvertarame Tamaitsainarpoktauk pane

Sorlo kiksarvingmêlaurame,

TaimainivoK ânanauvingme,

Ajulertut illûnaita

Nâpkigivait Jêsusima.

7. Kiksartose ajornisse Pivlugit, ôma kaikungmasse,

Sâtitse mânna ômunga, Illûnaita nâpkigingmagit. Assiokongimattille, Sôg kajusimavît sulle Kivgartorlutit ajortomik? Piulivlutille tikkimmat Ajornerit tunnulirkit! Jêsusib nâpkigingmattit.

8. Kailaurit okumaiguvit, Tikkinasuarille, unêt 
Ajornivit nênerpattit, Tikkikungne nâpkigivâtit. Ahâk ômattâ angmartor, Pagreartorpâtit ôma, Akkuneka rêmitingnermut

Uttakkivâtit $\overline{\text { pulivlutit; }}$

Kailauritse illûnase, Jêsusib nâpkigivâse.

9. Okarnæk: sappernarpunga Akkunit attornerlukkapkit

Saimarsautingita pingit, Kaikungmanga nachogigapkit.

Taimainæk! Nellâgut mânna

Sænnikteriguvit illa Ajornernik, kugviornermut

Kennuvigigungne ômamut, Aungminut ubvalerlutit, Jêsusib nâpkigivâtit.
10. Okarniarminæk: „sulle "Tuavitsariakangilak, „Nunab allianarningit "Attoromakârpakka sulle; „Uvlome nâpkiningnine "Gûb sorêrniangimago." Aukak, nipliangmat urlome, (Ebr. 4, 7.)

Piulijomavlugo tarnît, Tussaruk, assiokonnago Jêsusib nâpkigingmago.

11. Illannâk ajortolingnik Illingnut amulaungmittigut!

Illingnut kêmitingnermik Illumertikit ômættivut! Ômættillo sêksimajoK Uvaptingnut sakKertilauruk

Kanimaniptingne tarnekut. Noккajuitilauktiguttôk Okarungnarkârtinnata Jêsusib nâpkigivânga.

KAKKIALERNERMIK PIULIJAUJOMANEK PITJUTIGILUGO.

215. Mel. 208.

Wir sind alle Sünder. 225.

1. Ajortoksôvogut Ominarlutalo, Gûd nâpkiniktor!
Idluitorsuit

Kappianarninginik Pêjaijôvotit. Ajungitotôvotit; Asserorsimmavogulle Inûlerniptingnit. 
2. Jêsus tokkonernut Ânianernullo

Piulilaunga!

Aungnut ajornimnit

Idluinimnillo

Ubvalaungminga!

Piniarnerijattit

Piulijaujutigivakka.

Tættigidlarapkit.

\section{Mel. 22.}

Herr aller Weisheit Quell. *325.

1. Illissimajotôjotit, Sillainera Kaujimavat, Igvit ikkajungikumga, Sulliniatsængitunga.

2. Tussakit tuksiarnikka Sillatunermik pitinga, Illitarкsilerkovlunga Illingnik nangminimniglo.

\section{1\%. Mel. 159.}

Imgerut 51.

1. Gûd saimarsaijotôgavit

Nâpkigilaungminga!

Pêjakit idluinikka

Epkêjarlungalo

Nâpkiningnît angidlarmat, Idluitullilaungnikka

Kigligintigivakka,

Kiksautigigapkit.
2. Ajortoliksôjunille Erniangogama, Sângne nachonadlarpunga Idluitullinimnut; Tâksiortuinalaurama, Sillatunekartiminga, Kaujitiniarlungalo Miksekârnernigle.

Schaff in mir Gott ein Herz. 551.

3. Gûd sennalaurit illumne

Nutâmik ômamik;

Nutâmiglo anernermik Nellâgôrtomiglo

Tunnitsivigilaungatôk! Kênarnit pingujarnanga, Anernîllo ajunginer Uvamnêtsainarle!

\section{Mel. 82.}

1. Inôjut akkornganne Idluartokangimat, Kivgakullutuinaujogut Errartorniaratigut, Saimarsainit pivlugo Tussalaungmitiguttôn!

2. Illumne anernera KappiaktoadlarpoK, Ômattiga serkomipor Ajornikka erkarapkit, Idluitullinikka Asserortigigapkit. 


\section{piulijaujomaner pitjutigilugo.}

3. Illa idluinikka, Ômigidlarapkille Tâpkonangat piulinga, Killamiglo tussarminga, Sâlagijaudlartotut Illingalerkonnanga.

4. Killamik saimanernik Tussartilaungmingatôk! Tættigigapkit Gûdiga, Aркоmik attoræksamnik Nellojungnaitilaunga, Killanârvigigapkit.

\section{Mel. 141.}

1. Ajokertulaunga!

Kuviagijarnik

Piniarkovlunga,

Gûdigigapkit;

Illa Anernerpit

Tessiorlinga!

Arvertarkovlunga,

Аркоsinerne.

2. Nekkorsitilaunga

Attît pivlugo,

Kappianartomit

Amulaungminga !

Kivgarigamgalo

Idluinikka

Kappiagijakka

Nungutilaukit.

\section{Mel. 36.}

Hier liege ich, o Jesu! Dir zu Füssen. 226.

1. Ahâk Jêsưse! sângnut pâmakpunga
Ômattimnelo sænnikterivlunga,

Naglingnerniglo akkêngaidlarama,

Nâpkigilaunga.

2. Aungnut ningautsaujungnaitinamgale

Kovilaurangne senningajolingme;

ToKkonît, âniatillo pivlunga

Erkarniakit.

3. Ikkitit pivlugit ajæktornanga,

Pijæksauniptut akkiniarnanga,

InôKovlunga, tokkomut illingnik

Tunnilauravit.

4. Tagvale nertutsainaromavagit

Kujalimullo nâlegomavlutit

Attîllo ussornartor sorairnanga

Nertudlarpara.

\section{Mel. 83.}

Das Vernunftlicht kann das Leben. 227.

1. Sillatunima illa

Pikarluktituinarpânga, 
Kaumanersoarminut

Jêsusib Kaumaksarpânga; Inûlidlalermanga

Serridlarpok tarniga.

2. Jêsus ijekartinga,

Tækkotsiarungnartungnik;

Kappianarmat illa

Târsiorner kaumajome

Kaumajok tækkojaungipat Uvlômariktillugo.

\section{Mel. 149.}

O! wo soll ich fliehen hin? 228.

1. Namut remmâjuksauvîk?

Kia piulivângâ?

Kialo pêjarkagit

Agviarutikka?

Igville Jêsuse

AklujoK sangêtor

NâpkigilauruktôK.

2. Tarniga tækkosaruk, Kellaksorsimajok, Ârinermik sappertok;

Killingit tautukit;

Jêsuse, saimaunga,

Kennuvigigapkit

Mannigorvigilagit.

3. Omattiga sittijok Aumalertilauruk, Kibliktaule, Kuginut Perkovlunga sângnut. Najormît uvamnik;
Pêkit ajornikka Pitsartutingalo.

4. Ikkitit kaitsingmatta Inûlijiksamik, Itterasuarpakka; Ajornikka pivlugit, Kappiasukkama, Aukpit pêjarligit Tamaita uvamnit.

\section{Mel. 132 a.}

Aus tiefor Not schrei ich zu Dir. 229.

1. Kiksadlarnermut Gûdiga! Kaigarsugvigigapkit, Tussaruk tuksiarnera, Siutikik mannikik, Tamarkattalauramale Idluitulligamalo Egitæksaudlarpunga.

2. Tagva Gûd tættigivara, Nangminimnik pinanga. Окаusingit malliklugit Saimaninga tarnima Tungavigitsainadlarpâ, Mannigorvigilugolo Nerriugitsainarpâ.

3. Unungmut kiksadlaruma Ama uvlarârsungmut, Nâlekab pitsartuninga Trettigijomavara. Illûnatiglo okpertut, 


\section{piulijaujomaneк pitjutigilugo.}

Erniangomajut illa,

Uttakrivât Gûditik.

4. Tâmna inôtigivavut

Apkutigællugolo,

Sæglotuitigivavut

Nagligidlarlugolo.

Nangminer imak okartor: „Uvamnik naglingniktu ble "Orausikka mallikpait.“

\section{Mel. 75.}

Wo soll ich fliehen hin. 230.

1. Namut sâniarpîk?

Nênertaudlarama

Ajortovaksoarnut?

Kia piulivângầ?

Inungne kiksarnimnik

PêjaijoKatsængilak.

2. Nâpkiniktôgavit, Tagga tikkipagit

Jêsuse! kaikogamga.

Sænnikteridlartunga

Tarniga nâpkigiuk,

Nekkorsitilugolo.

3. Kittorngarigamga, Ajornerijakka

Kappiagidlartakka Illingnut pitipakka, Pêjalaukit tokкungnut. Inûlinga ikkingnut.

\section{Passijæksaunane} Aut kovilaurangne,
Ômunga ubvarminga! Mannigoruk illuga, Ajornikka tamaita Erkaumajungnailugit.

5. Ullapirsartiga, Piulimagamga, Idluitullinimnik Illa pêjaijôvotit! Illuvernut makkoa Parngnanêrsokit sorlo.

6. Ajornikka illa Unnuktorsôngmatta, Aungnulle pêjalaukit, Pivlunga tokkogavit. Illinguik nellipsaijut Tamaita âkigangne.

7. Inûlijaunerma

Pijariakarninga

Sokrosingilak sulle, Aut kôktor ikkingnille Pitsartutigivara Sâlagilugit ajortut.

8. Jêsusiga aggut Pitsartuneкаrрок, Taimak aukpit kissime Inuit illûnaita Ivsornaitidlarlugit Piulijungnarmagit.

9. Tamanna pivlugo Mânna ômativut, Tamaita epкejakit Aungnut ajunginermut; 
Illingnullo torkokit Sapputitsainarlugit.

10. Gûde, Anernernut Tessiolaungmiuk Kibliklugo illuga, Allaijungnarkovlunga Avinartunik illingnit, Ungagitsainarlagit.

\section{Mel. 132 a.}

Stärk' mich mit deinem Freudengeist. 231.

Jêsuse najulaungminga, Inûlinga ikkingnut, Kidjijârnernut ubvanga Illungertuleruma, Angergarkojomârumga Annerijarnut ailanga, Najugarnêkovlunga.

\section{Mel. 168.}

Herr, ich habe missgehandelt. 234. Nâlegar pitlarnangatôk! Ajortullilaurama

Perkojarnik mallingnanga Kaujitigalloaramga. Ajornima nênerpânga, Tailugille sapperpunga Unnurningit pivlugit, Igville pêjalaukit.

2. Âniagavit Jêsuse, Aungnut inûlilaunga, Ajornikka pêjalaukit,
Ubvarlugo illuga Idluitullinimnille; Ikkingnullo itterlanga, Siorniudlarnimnit Piulijaukovlunga.

3. Pêjaivigijungnarparma Ajornimnik Jêsuse, Egikit immânut mânna, Ivsornaitilaungminga. Anernerpit pairilinga, Illingnêtsainarkovlunga, Tessiudlarlingalo Axsârnigaukonnanga.

\section{8\%. Mel. 75.}

Herr Jesu, ewig's Licht. 235.

1. Kaumajor Jêsuse! Gûdemit pijotit: Illumertittiguttôk Naglingnermik illingnik, Perkutaumarikluta Illingnênarkovluta.

2. Nutângortittigut Jêsus kaumanernut, Kaumajome-iluta Arvertarkovlutale, Gûb kittorngangititut, IdluarijarnuttôK.

\section{Mel. 123.}

Erleucht' mich, Herr, mein Licht. 237.

1. Kaumaksalaungminga Illitaringinama 
Uvamnik, Gûdiga!

Sulle sâgiarnerma

Nâmangininganik

Mallugosukkama;

Igvit âkiminga.

2. Sannikterivunga

Naglingnera nellâgut

Illingnut Gûdiga

Pingimat, illumne

Ajortôjungale;

Tagva ajornikka

Kiksautigivakka.

3. Ômattîgle mânna

Sâgiarit nellâgut,

Nekkorsinginama

Kristus perkârnago,

Ikligunikkalo

Allarmarikupkit

Nâmaksidlarpunga.

\section{Mel. 125.}

Herr Jesu, Gnadensone. 238.

1. Sekriniodlartotit

Nâlegak Jêsuse,

Inôsiojotillo

Attê ồmatiga,

AkkisuktilauruktôK,

Natângortilungalo;

Gưdik tussalaunga!

2. Tarnimnit annitikit

Uvingub issumangit, Annerosuktilunga
Illingnik Jêsuse !

Inôniarkovlunga

Nakorijarnut mâne,

Piulijaugama.

3. Pigannertilaungminga

Illitarivlutit,

Illissatilungalo

Ajokertûsernut:

Illingnut Nâlegara

Okpitsainarkovlunga

Ungagênarlutit.

4. Naglingnît Nâlegara

Pititsuk tarnimnut,

Ômamuttauk illingnik,

Naglingmerkovlunga,

Kuviagijarnullo

Arvertatsainarkovlunga Apкotingne illa.

\section{Mel. 182.}

Ich kriech', Erlöser! Dir zu Füssen. 240.

1. Piulijiga! pâmakpunga Ittigangnut, nâpkiginga, Ikkitit kunnitsainarlakka Pêjakillo ajornikka; Tâpkoa unnudlarningit Kittiksaungitôgalloarput. Ivsôjunga ajæktornanga Ajortuggit piulijingat.

2. Ikkingne inniksakarpunga, Nênermanga ajornima; 
112 Kaкkialemermik piulijanjomanek etc.

Ómatinnik inûliklerte Pêlaukit âniaksakka. Uvamnik nachungitotit,
Piginga okpervigigapkit; Tammarsimajotun-inama, Sennerarnut pitilaunga.

\section{OKPERNERMIK JESUSEMUT.}

\section{Mel. 36.}

Ach mein Herr Jesu. 241.

1. Nâlegak Jêsuse pigingikupkit,

Aggullo tuksiangipat pivlunga;

Ajortunit ajortûnerôjunga Namut sâlungâ?

2. Kappiasungnermut namut ailungâ?

Omatiktut naglingniktoKarnerkâ?

Assingnik tætteKarnangakissivit

Pituarigapkit.

\section{Mel. 165.}

Durch des Heiland's Blut und Leiden. 242.

1. Piulijipta aunganut Ânianingauullo

Kivgaujungnaititauvogut, Ajorniptingnit illa. Gûb nagligosungninga Nertornadlarningalo Ômatipta opigivâk. Ullapkuteкalerapta.
2. Nênertaujutiksaptingnit Piulijaudlarpogut, Piulijipta ikkingit Kemâvigiguptigik; Sokrosijuiluta Okpervigiguptigo, Pairiniarivâtigut. Perkutigimarikluta.

3. Jêsuse tækkkonârupko, Assingit illûnaita Kamagijungnailerp akka, Tamna pijomagapko. Naiperkotigingmanga, Pijariakartakka, Omatimalo piksangit Nagvârpakka tâpsomane.

4. Perkutigijomavara, Najoromavlugolo, Piniarnikka tamaita Tættigitsængilakka; Jêsusible Kaitangit Nâpkigosungnerminut, Inôgutituarigapkit, Opigosûtigivakka.

\section{Mel. 37.}

So lang es Gott gefällt. 243 .

1. Gûdib kittorngangit InôKojangit 


\section{Okpernermik Jêsusemut.}

Nunamêtillugit

Ajutsainarput;

Okpertorle ôma

Nâpkigosumut

Asserorkongilâ

Piulivâle.

2. Illitarigupta

Nangminiptingnik,

Illuptingne tagva

Ajudlartomik

Nagvâtsainarpogut;

Tarne timelo

Asserorsimavuk,

Ingergarmigle.

3. Jêsuse saimavok

Kennuêlune,

Okautiguptigo

Ajorniptingnik,

Mannigôtiksavut

Tagva Kannipoк,

Aungminut tarnivut Inûlingmagit.

4. Kugvit allaterpait, Pairilutalo,

Merngoêrservingmut

IIlijaukâ.rata;

Timit sutaertuksat

Ivjume aglât,

Tæettekartilerpait

Adsigilugo.

5. Tamanna pivlugo,

Illa tarnima

Pituaridlarpâ
Piulijiga.

Kilangmik nunamik

Kamangilanga,

Najorninganele.

Itsainaruma.

6. Gûd ping stitiga

Najulerpara,

ErKaumadlarmanga

Kujagivara.

Aungminullo ôma

Piulivânga,

Tokkotigivânga

Inôkovlunga.

\section{Mel. 132 a.}

Such', wer da will, Nothelfer viel. 244 .

1. Piulijiksaungitunik

Kennertut, sullingilat:

Mâne attausênaujomik

Piuliklertokarmat.

Ôma piulivâtigut

Idluarsarpâtigullo

Pivluta tokkolaurame.

2. Pulijaujomajose

Tâmna kenndlarsiuk

Gûd nâlegaujor kissiat,

Piulijigigapsiuk

Tâmna kemnitsainarsiuk,

Piulijanjok ômunga

Ajoksangimat illa. 


\section{4 a. Mel. 166.}

Es ist vollbracht, was willst du nun. 245.

„Nâmaksivok!“ piulijaunît NangmineK sapperangne,

Piniarnernut ajornitit

Pêjarungnanginangne. „Nâmaksivok!“ kammagiuk, Akkilêkongilâtit,

Okpernermêtuaruvit Tamât inôtillutit.

\section{Mel. 146.}

Herr ich bekenne mit dem Mund. *349.

1. Nâlegak, ômamut

Kigligiudsivunga :

Piulijiksamik

Assingnik pekangimat.

Attilaurangnelo

Uviniga augalo,

Tamanna pivlugo

Ningaringilarma.

2. Aut pivlugo кilak Angmartaungmat pivluta, Tagva okperpunga: Gûde nâpkiniktotit, Ômamut okpertor ToKk Allarniængilat Tokroviksanganut.

\section{Mel. 22a.}

Der Glaub' wirkt im Gewissen Fried'. 246.

1. Okpernerub saimarsarpait, Kiksartullo mannigorpait, Gûd nertorpâ, tâpsomunga Kittorngautitaulaurapta.

2. Kujagivavut Gûdivut Okpertungortimmattigut, Ernermigut Kristusekut Piloreutigivavut.

3. Okpernermut Jêsusemut Piloridlarungnarpogut: Tâpsomunga sâgiartor Sapputijautsainalerpok.

\section{3\%. Mel. 15.}

Gleichwie sich fein ein Vögelein. 247.

1. Sorlo nuname ômajut Sapputsauviksamingnut, Pullangmatta sillarnerlukpat

Torkortaujomalimut:

2. Taimaktauk pullasârpunga,

Ikkingnut, Jêsusiga! Ajortut erssisârppanga, Sapputsaujomavlunga. 
238. Mel. 168.

Jesu, der Du meine Seele. 248.

1. Jêsus tokKungnut Kejungme

Tarniga piuligangne,

Sâtanasib najugânit

Tâktomit amugangne,

Ajokertorluaramga

Inônasuarkovlunga,

Nâksaungitomut, tagra

Okperlualertinga.

2. Jêsusiga pêjarpattit

Idluinikka aungnut;

Piulijiga tamanna

Piulijautigilago!

Anialutit ajortut

Nangmalaurangne $\mathbf{k e}-$ jungme,

Ajortoêrtilunga

Pigitsianga tagva.

3. Jêsus auktojut ikkivit

Kikkiæktortaunivit,

Puvinivit annautamut,

Illa tigluktaunerpit

Ómavît kappijauningat,

Timivillo aungêrotub

Kuviasuktipânga,

Mannigortidlarmanga.

4. Ikpigijakka tamaita Igvit Kaujimavattit; Anianartok tokkonît Innekarle tarnimne. Omattiga kiksadlartor Aungnut kovisimajomut
Kejungme, serparsaruk, Igvit, Jêsuse piuk.

5. Nâlegarle! okperpunga, Innertuijungnaitinga. Nekkoksititsungnarparma Ajortut tikkippanga. Nâlegaujoksôgaville Tættigitsainadlarpagit Tautulerkârtinnanga Illingnik, Jêsusiga.

\section{Mel. 22a.}

O Jesu, sieh Dein armes Kind. 249.

1. Ahâk Jêsuse! kittorngat Ittiggarnik erkitsijok, Kiksarmat tækkosalauruk TokKovîllo kiblikliuk.

2. Nâpkigijæksaungitunga Puailavingnut pivunga, Ômattiga sangêdlartor Aungnut inûlilauruktôr.

3. Ajortoksôvunga illa Ajortulle Kaujivunga Pêjadlalaurangne igvit Pivluta tokkolauravit.

4. Tarnikkullo timikkullo Ânianît tautukkapko Kidsijâdlarnîllo aungmik Kappiaktoadlarnermut;

5. Niakôsidlartaunîllo Mîttautigijaudlarnîllo 
Issakajaudlarnîllotauk Kollêt nerkerksortidlugo;

6. Kejungmut kikkiæktortaunît

Sêrnartomik imitaunît, Aunâdlartullo ikkitit.

Kassilinadlartor tokкut;

7. Tamakkoa illûnatik

Pivlungatauk pilaungilat? Aukak illa Jêsusiga! Tautudlarpagit pivlunga.

8. Opigomut erkipagit, Aunârlutit nivingagavit. Aumavor illa illuga, Taimak nægligidlaramga.

9. Atâtamut oKaravit:

Ivlerinago inôtsit TukKotigidlalauramga, Takopsoagidlarpânga.

10. Abba Atâtamnik! mânna Tailerlutit sappernanga, Kittorngarigamga illa, Einerpit aunga pivlugo.

\section{Mel. 183.}

Sei gnädig Jesu! voller Güte. 250 .

1. Nagliktor Jêsuse! saimaunga

Ômatimuut kenıugama. Saimanernik kilanârpunya,
Saimaunga, Gûd, ajorama! Illa ajæktutsængilarma, Saimarlungale pivlutit, Piuligamga ajornimnit, Aut kovidlarangne pivlunga.

2. Ômatipkut erkila ulagit, Innigilauruk illuga.

Kemmalaurangne ânanaunît

Naglingnerınut uvaptingnik,

Ômatiga nacho ginago Annerilauruk iglungnut, Timigalo ajulerpat, Tarniga kongmuartilauruk.

3. Okpernermut aivigivagit Naglimut akkarviginga! Illumertilaunga illingniik Kuviasuk vilungalo. Kujagitsainaromavagit Omattib ômaningane, Tiglerniarungnaipallo Soraertuksaungilak ninglingıек.

\section{Mel. 211.}

In der Welt ist kein Vergnügen 253

1. Tarnil sattôt ksanganik Nuname pekangilak Jêsusib assianik, Ajornernut sìlagijak 
Uivêrivok ingminik Jêsus unganartotôngmat, Ajoksartub tarningat Mannigortigidlarpâ.

Sông-o-voK

Nagligosuktorsôninga

Pilorikput tâpkoa :,:

Jêsusib illagêngninganik illangiusimajut. :,:

2. Tarne assiojuksauvor

Idluarkôrpallônêt,

Jêsusib piniarninga

Tættigingikuniuk,

Annorârinagolo.

Kangusuktitaujomârput

Idluarasugijut,

Epкêjarsimanatik.

Tai-mai-mat

Ikkingit tættigissigik!

Piulijauvut tâpkoa :,:

Jêsusipta ikkinginut autnârtunut torâdlartut. :;

\section{Mel. 4.}

Am Tag des Gerichts. 254.

1. Tikkiviksarne

Jêsuse sângne

Tækkuksaulerpoк

Ômatiga aukpît ubvalaungmago.

2. Jêsuse sângnut

Pâmakterpogut,

Opigosumut

Piuligaptigut nakorivlutit.
243. Mel. 146.

Dort im Vollendungs-Saal. 255.

1. Takpâne kilangme

Idluartut inningænne,

Komunionemut

Jêsub attortanganut

Tâpkoa kissimik

Illaujungnalerput

Epkêjarsimajut

Jêsusib aunganut.

2. Tagva illûnatik

Gûde kujagidlarpât,

Ikkinginut illa

Mâne âkiksimajut.

Naglilaukpissingâ?

Appertsorpattigut, Kugvinut: Ahaila!

Kioniarpavut.

\section{Mel. 106.}

Ieh habe nun den Grund gefunden. 256.

1. Okpernima kissarviksane Pêjuitok nagvâlaukpâ; Illa Jêsusib ikkingine Kiglinganit tagvanêpoK; Kissarviksarle sôngojor, Sillaksoak nungulerpat.

2. Issumalinangitor Jêsub Naglingninga nagvârpavut, Aggangillotauk issammijut 
Tussumut ikkajorluta. Naglimut illungertorpor Uvaptingnik kaikojimut.

3. Assiokonginamittigut Gûdib ikkajorpâtigut, Tamanna pivlugo nunamut Erningat tikkipâtigut; Taimaimallo ômattivut Pattaktorpait tupaklugit.

4. Naglingniub nelleкangitub

Ajornivut pêjarmagit;

Kristusib aunganut illuvut Nellâgut inûlijauvut; Kristub aunga nipliangmat „Nâpkinermik! Nâpkinermik!"6

5. Naglingninganut aumavunga,

Aungminut piulingmanga; Omatânik tækkunârlunga Ajornimnik kiksaruma, Tagva nagvâtsainarpunga Takopsoagilermanga.

6. Kissarviksak una illâle Najuênaromavara, Naglingninga inôtillunga OKautigivlugo tagva; Takpânelo sorairnanga Nakorênaromârpara.

\section{Mel. 11.}

Jesus ward ein Mensch für mich. 257.

1. Jêsusiga uvaptut

Pivlunga inôlermat,

Tarnima serrigivâ

Nekkoksitigigapko.

2. Jêsus kemmergogupkit Nerchevingmêlaungnernit Illuvermêlaungnernut Okperlunga illingnut.

3. Ajulernera tagva Erksinartullo illa Tamaita nukkingêrput, Jêsusiga tokkungnut.

\section{Mel. 29.}

O inniglich geliebte Liebe. 261.

1. Nagliktiga ungagijara, Naglimutnellerangitomut, Tâktomit nussulaukparma Kaumajomut pitilunga.

2. Ajornimnut tokkungalunga

Illumne asserulaukpunga, Ingergât ikligunima Sâlagikattalaukpânga.

3. Ômamut illapsugivarma, Uvamnik asserortigama, NangianartoKsoarmut Kattaksimadlalaurama. 
4. Naglimut amualaukparma, Ajornerub pitsartuninga Serkomipat, pitilunga Ullapirsautingnik sângne.

5. Mânna nagvârpunga ikkingne

Nâmagijaunimnik Gûdemut,

Saimanernut illauvunga Gûdib annerijanginut.

6. Ittiggangnut sêrkortorpunga,
Kujalivigijomavagit, Piniarnitit pivlugit, Serrimut kugviorpunga.

7. Inôtsimne tamât nuname Aggangnut tessiulaungminga!

Arvertatsainarkovlunga Nertornautiksarnut mâne.

\section{4\%. Mel. 16.}

Süsser Heiland deine Gnade. 262.

*1. Nâlegak, nâpkigosungnît Issumagijaptingnit Anginersaungijaidlarnoк, Illa tattamnarpotit.

2. Tuppaktor, ajornerminik KiksartoK, Kristusemut Sâlerpat, tuavitomik Âriktauvor aunganut.
3. Jêsusiblo ikkinginit

NâpkiningneK kôlerpor;

Sâgiarame, Abbamik

Nipliavok okpertor.

\section{Mel. 79.}

1. Uviningmêlauravit

Nâlegak, okpernermut

Tokko sâlagivat,

Sillaksoarmiullo

Piulidlalaurangne,

Okpernek sakkogilaukpat.

2. Okperlunga illiktut Árvertartuksaugama, - Unêt sangêdlungaOkpernek tiguvara, Sorsugiarkogamga, Ajornek sâlagilugo.

3. Okpernermik illumne Pigiarnilauravit, Nâmaksitiminga! Gûb nâlegauvinganut Itterungnarkovlunga, Naggatiksamnut piguma.

\section{Mel. 121.}

Das Lämmlein ist geschlacht. ${ }^{*} 374$.

1. Jêsuse tokkungmat,

Sillaksoarmiut

Piulijaumavut;

Tâpkoa kissimik

Assionialerput, 
Kaijomangitut

Jêsub aunganut. ::

2. Jêsus tarniptingnut

Sakkijalerângat,

Tokkojut ômarput

Kaumarput tâktomêtut.

Ânertaungmat Gûdivut

Kemmergovavut,

Kuviasukpogut

Piulingmattigut.

\section{Mel. 75.}

Ach Blut, ach Lämmlein'sblut. *376.

1. Saugârsûb aunganut Âriktaulerapta, Opigosudlarpogut Ikkinginut pigapta; Nâpkigijauniksavut Taijaujungnatsangilak.

2. Pivlunga Jêsuse! Tokkotaugaville, Sungertûtigivagit. Sapkunginamga igvit, Kittorngarilungalo Illingnik pigamalo.

\section{Mel. 167.}

Ach Jesu, meiner Seelen Freude. ${ }^{*} 377$.

1. Jêsus! kuviasûtiga Akluijutigalo
Ajorsarvimne tamâne ;

Pituaridlarpagit.

Ômisukteolaurpunga

Illingnik, igvit tagva Aungnut aglât inôsera

Tokкomut piulivat.

2. Kaujivunga Nâlegara! Inôtigidlarapkit, Pivlunga tunijaujotit; Illingne Jêsus! mânna, MerngoêrserneKsivunga ; Koaksârtitaugaiguma Ajortunut, sunaninullo, Illingnut Kemâvunga.

\section{Mel. 22a:}

o Jesu Gottes Lämmlein. *379.

1. Jêsuse Gûb saugâr'sunga, Aggut kissiat tokкullo, Piulijautigivavut, InôKovluta illingnut.

2. Kilangmit avimajogut Illûnata kattangnermut, Piulijiksakarata Tokkomit Sâtanamillo.

3. Gûd tikkipaptigut tagva, Inôtsit tunnilaurangne Tokkomut, piulivluta Kappianartunit tamainit.

4. Tokkogavit! Gûdib aunga Kôktor timingnit, ômunga 
Sillaksûb tagva inungit, Tokkomit piulivattit.

5. Gûdib ningausinga mânna Kammingmat, Sâtanaselo Sâlagijaungmat; Gûdemut Erniangojungnarpogut.

6. Tokкut okpervigivavut, Nâlegatuarivlutit; Aungnik annorâkarapta, Toкkо erksijungnaipavut.

\section{Mel. 244.}

O Jesu $\mathrm{Du}$ wollst geben. 265 .

1. Jêsus, inukotitit

Tunnitsivigikit

Okpernermik ômame Kaut tamât nutâmik; Aungnullo tokrungnut,
Tarniblo nellâgut Ungagitsainarlitit Pituangogavit.

2. Kanoktôk âniavit Illagilittigut, Kejungmelo tokkonît Ômamigilavut Sapputilauktigut, Ôktornartovingme; Kuviagingitarnik ErKsilerkovluta.

3. NâpkiniktoKsônîllo Missigidlarlavut; Tamaptalo saimanît Peкarvigilavut. Illanârijivut Tækkonârmitigut Saimanernut; nellâgut Okpilerкovluta!

\section{AJORNERNIT ISSUMAGIJAUJUNGNAINERMIK.}

254. Mel. 155.

Gnade ist ein schönes Wort. 267.

1. Saimanek ânanauvor Ajortoliksônermingnik Kaujijunut, Ikpigidlartunnullo: Ingergât Illinganerilauktattik Amujaulerkârtinnagit Assionadlalaungmat.
2. SaimaneK angidlarpor Ajortolingnut tamainut, Jêsumullo

Mannigortaumajunut;

Tâmnalo

Aiviginiarunitsuk, Illalliortautsainarput Jêsub nâpkigingmagit.

3. Nâpkigijaukârata, Kigligiudsilaukpogut: 
Jêsusiga!

Tâktoksoarmêpunga

Nâpkiginga!

Tagva inûlivâtigut

Idluarsitilutalo,

Piloridlarkovluta.

\section{Mel. 228.}

O Gnade, sei mir täglich neu. 268.

Saimanartor pilauktara Jêsusima kailauktânga,

Tukkisitsainarlago!

Tuksiarvigilaurapko

Tâpsoma oKautivânga:

Pivlutit âniagama,

Inôlerit, ajornitit

Pêjarlugit, illûnaita

Issumagijungnaipakka.

\section{Mel. 164.}

Sagt an, die ihr erlöset seid. 269.

1. Piulimajose mânna

Okaritse : nakille

Nekkoksitiksakarkise,

Serridlarungnarapse?

Jêsub tokkunganit

Aunganillo pivavut,

Naglingnermut ajornivut

Ingminut illilaungmagit.

2. Uviniptingnik attivor Tokкojungnalerlune, Inuglo assiojuksar
Piulijaukovlugo;

Mânna Jêsusivut

Pairijigivavut.

Tâpsomungalo pingitok,

Nekkorsitiksakangilar.

3. Gûb Anerningat kissime

Piloriktipâtigut,

Inôtilluta nuname

Tessioramittigut

Jêsusemut illa;

Illagêt tamaita

Kristusib naglingninganik Illumertênaramigit.

\section{5\%. Mel. 14.}

In evil long I took delight. 333 .

1. Ajortomik akkuneкa

Kuviasuktunga,

Kanguginago erksinanga

Koaksârnangalônêt.

2. Tækkogama suilamnit

Koaksâtainarpunga,

Inôserivalauktara

Nokkartitainarpâ.

3. Kejungme nivingavlune Kappiaktoajoк, Aunârtorlo angijomik Illuma tautukpâ;

4. Ijingminut sangêtungnut Tækkonâraminga 
Kejungmit seningajolingmit

Kannidlartillunga.

5. Anernangêrkârtinanga

Puigungilara

Ôma tækkonârningata Kiblidlalaungmanga.

6. Illumne passijauvlunga Tokrotilaurapko,

Taimak kaujititauvunga Tokkungmat pivlunga.

7. Tautukama : ajornima, Aunga kovidlarpât, Kikkiaktoramitsugle Tagvane kejungmut.

8. Sullinimnik nellolaukpunga,
Kugvikka sungilat; Namut sajuktor tarniga Ergutijungnarkâ?

9. Ama tækkonâraminga, Imâk oKaupânga: „Issumagijungnaipakka „Ajornitit illa.

10. „Pivlutit korlorpoк auga „Pissiarivlutit, „Inôkovlutit pivlunga "Tokkotigivagit.“

11. Kangusûtigijæksakka Ajorniâlukka
Tokxungat sakкertimmagit

Kakkialerpunga.

12. Saimaningat angininga Imaipor uvamnut, Issumagijaujungnainimnik Kaujitidlarmanga

13. Tokkominut saimarmanga

Mânna ômattipkut

Illumertautainarpunga

Kuvianartomik.

14. Jêsusima inôtsine

Tunningmago pivlunga,

Mânna inôvigivara

ToKkotigingmanga.

258. Mel. 146.

O Jesu voll Geduld. 271.

1. Kennuêsârtotit

Saimaneliolutit,

Nagligosungnernut

Umatiga kibliuk!

Ikkajungikumga

Mannigoijomik

Nellomarikpunga

Sillarsoarmelo.

2. Sâvigivagille

Mannigorungnartiga,

Illannâk ômatît

Tækkonârungnarupko; 
Tagva saimanermik

Ullapirsaumiglo

Nennisilerpunga

Kênavît sângane.

\section{Mel. 83.}

Herr, mein Heil in aller Angst. 272.

1. Jêsus Piulijiga!

Kappiasudlarangama,

Ki ejungmut ârlorlunga,

Ikkitit remmergovakka;

Taimailiorangama

Munnigortaudlarpunga.

2. Tagva tautuktiparma Ômatingnik angmajomik, Oкautilerparmalo:

„Kait mannigoromagapkit, "Nênertaujutiksatit „Tоккumnut pêjarpakka.

3. "Ômatît kappiasukpat "Ikkimnut unnertôtiuk, "Piuliklernek taiguk "Tâpsomunga, kailauktara;

„Mannigotuinarit,

"Uvamnut attalutit.

4. ,Taimak illioruvit

„Erkartortautiksaerpotit, "Ajornitit pêrapkit „Piloriktisimavotit; „Aniajutigigapkit "Pitlaræksaungilatit."
5. Mannigornarpor ôma

Nippinga nellungitara,

Kaujitimmanga sulle,

Ômattinga illapsumut

Tættæmat naglingnermik;

Akitsainarmangalo.

6. Âniatigingmanga

Aunârtoksôngmat pivlunga,

Takpâne sorairnanga Hallelujâromârpunga; Tussarnidlarpok tagva Kujalidlarniksara.

\section{Mel. 234.}

Vor wahrer. Herzensänderung. 275.

1. Illunarsoatik inuit

Ingergarmik ajortoksôvut, Inutokat inûsuktut

Ningarijæksaukattigêkput. Gûdible tillilaungmago,

Ernine, inogutaukovlugo, Tagva kigligiksarupta Idluiniptingnik sângane, Jêsuse nellâgut Ikkajortigivavut. Inugle sâgiaromangitor Piulijiminut,

Okperviginago Piulijaujungnatsangilak.

2. Saugârsugle tokкotitak. KujanadlarpoK nâpkiningnît! 
Naglingniktorlo uiksar

Tarnipta kuviagivâtit,

Pivluta kappiasuktotit,

Timillo âniatitaujok

Pivluta tarningêrtotit.

Sennerkakut kappijaugavit,

Torârtimitigut,

Jêsuse tokkungnut!

Tarnipta sânginêtsainalaungmît!

Allarnagolônêt,

Kristus Gudeongmat

Pitlautiksaptingnik pêjaijoK.

3. Opigitsainarlaptigit, Jêsuse pêjaijotôjotit Ajorniptingnik! Igville Inuit piulingikungne Saksârajatsainarpogut. Mânnale siorniornıvît, Aungmiglo kidsijârnivît Aukjîllo, ikkivîllo toKкo-

\section{vîllo}

Kappianartovingmit

Piulivâtizut.

Akkiliutiksakanginapta

Pigilaungmitigut,

Piulikl rnernut

Pitsartutilaungmitigullo.

\section{Mel. 97.}

Du unser auserwähltes Haupt. 276.

1. Niakuk, Gûd pairijivut, Tarnipta okperijangat;
Jêsus, annerimanivut

Jkkingne remmergolavut!

Sennerkakut angmartitaujokut

Tarnivut tessiutsainarmigit.

\%. Tamanna tattamnadlarpok

Sorrutsinut okikôrpok, Inûble okperilugo

Ajorkôrpâ tokkominut;

Ômunga illagêt Kaujijauvut

Killangmiullo imgerutigivât

3. Sillaksoakarningane

Piulijivut Kristuse

Tungavituarivavut;

Okautsit tussarnertut Gûb Pitsartutingmagit kaujivasse,

MikseKârtomik raujimajose.

4. Nutaraunerminillônêt Passijæksaungitokarpat, Ajornerit nellîllônêt Sulle attungikunigit; Tamannalo tættigiguniuk, Sillaidlarlunelo takpedlarpor.

5. Asserorsimanerminik Illitarksimajokarpat, Piulijomagunele Ajornerminit ingminik, 
Pinasuaralloarunelo

Piulije illitaringilâ.

6. Assiojuksaukôrtorle Tokкomit tuppalerune, Idluitullilaungnine Kiksautigilerunigit, Piuliklerte sâvigilugo. Tagvainak tâpsoma nâpkigivâ.

7. Taimak illapsugijane Jêsusib okarvigivâ: „Issumangnarungnainermik „Ajornernit pillipagit; „Tækkonârlunga okperviginga, „Kuviasuklutillo makkitit."

8. Tagva tarne anernermik Nutâmik pillitaulerpok, Jêsub pijomajanganik Okpernermut pijungnarpok,

Nutâmiglo annorârsimaทоK

Saugârsungmik illitarksidlarpor.

\section{Mel. 151 i.}

In Sünd' war ich verloren. *394.

1. Assiomalaukpunga Ajor ksôngmatta
Piniarnerijakka;

Tagva Gû̉b erningat

Erniangupsârtipânga

TokKotaunerminut,

Piulijauniksamnik

Pinialaurame.

2. Ajornikka tamaita Mattumavut mânna,

Kangusuktitsungnaipânga Gûdima sângane: Annorârtitauvunga Pinnarnadlartomik, Piloriktitaulunga Id Iuarijanganut.

\section{Mel. 16.}

Wie der Herr am Kreuz gestorben. 277.

1. Nâlegak tokkolilaungmat, Tокко nukkingêrupoK;

Tagvalo pissiarivânga Kittorngaminut illa.

2. Kittorngaunerle Gûdemut KanoK tukkelik mânna? Kaut tamât nutâmik tarne Udsertorlugo illa;

3. Jêsus Kennuvigilugo Nutakratut ômamut: Gûd tessiutsainarminga Idluarijarnuttôk. 
4. Tagvale sannikterineк Gûd najoromavlugo, Anernermut nênertauvok Mânna ômatiptingnut.

5. Mattoma kugviortipânga; Illungertudlarlunga Tussugama, Jêsusemut Piulijaujomavlunga.

6. Tagvainak tokкotaulauktor

Ajornivut pivlugit Jêsuse ikkeliksôjor Sakkerpok tarniptingnut.

7. Taimailerupta tarnivut Jêsusib agganginut Perkutauvut, tâpsomalo Pairivait naglingnermut.

\section{Mel. 1.}

Was hätten wir für Froudo oder Ehre. *396.

1. Suna Kuviasugvigivittigo Gûdivut kuviagingikuptigo?

2. Passijæksaungitokarka inungne

Gûdib erkartuiviat sângane?

3. Kina piniarnerijane pivlugit
Pilorigungnarka Gûdib sângane?

4. Kinalo nagvarka apkut kilangmut

Jêsub ikkingita assiatigut?

5. Taimaitorangilak inugasængne.

Jesusênakut itterungnarpogut.

6. Sannikterijok Jêsusemut pikpat;

Ajorninelo pivlugit kiksarpat;

7.Nâlegapta tâmnale mannigorpâ,

Kittorngaminulloillautipâ.

8. Sâgiarangatta tammarsimajut,

Kuviasugvigivait kilangmiut,

9. Illagivlugit Piulijivullo

Nakoridlarpât kujagivlugolo.

\section{Mel. 4.}

Lamm für uns geschlacht't. *397.

1. Piulijiga!

Tokkotigigamga,

Tussaumagapkit 
Kiksadlalerpunga naglinginapkit.

2. Sillaidlartunga

Tautunginama,

Ajornimalo

Kivgarilaukpânga toKkungavlunga.

3. Kaumajub tagva

Najulerpânga

Tuppaktidlunga

Ilitarilugo Piulijiga.

4. Ômatipkulle

Takkordligama

Tuksiarpunga :

Jêsusiga takopsoagilaunga!

5. Sorairnangalo

Tuksiarapko,

Piulijima

Aungminut illuga âkiksolerpâ.

6. Iliumne sorlo

Tussalerapko

Orautimmanga:

Ajornitit pejaromalerpakkid.

7 Orausingalo

Okperigapko,

Ikpigivunga,

Aungminut ômatiga ubvarmayo.
8. Piulijiga,

Najuênanga

Pairilaungminga

Ikkitillo opigitsainarlakka.

\section{Mel. 164.}

O Tage wahrer Seligkeit.

1. InôseK illa nellâgut Kuvianarpok tagva, Pêjarpa ta ajornivut Mâne inôtilluta; Jêsuse pivlugit Âniadlalaungmat: Ullapirsautinganullo Ônarsikpatta illuvut.

2. Sannikterijor ômame Piulijiksaminik, Gûb ullapirsarmagole Aumavor angijomik, Missigilerune Piulijaugame, Tagva kilangmêpok sorlo, Jêsub mâne najormago.

3. Kanorle, taimaitok sulle Kangusumut reangmat? Kuviasudlarlunele Jêsub najorninganik? Ajornine illa, Puigungimagit; Mânnalo prulijaungmat, Jêsub pingmago aungminut. 
4. Tamakkoa erkarlngit, Taimaitsainadlarpogut: Jêsub piulingmattigut, l'ujagitsainarpavut, Nellopkotimullo Kuviasumullo;

Angijomik ajortogut Tokrotigilaungmattigut.

5. Pilloringnivut illale Orautaujungnangilar; Aungat epKêjarunitigut Inûlivâtiguttauk, Ajuleruiptingnit Pigivaktaptingnit Illa aungat torkungallo Piloriktênarpâtiguk.

6. Aungagut ikkingittigut Kilangmut itterpogut; T:imailertimmattigulle Amuluta ingminut, Pakkoa illagêt Imâk tuksiarput: Gûd, saugangalo nertornak

Aungat idluarsarmattigut.

\section{Mei. ㄴa.}

Christi Blut und Gerechtigkeit. 279.

1. Kristum aunga idluartok Omatimne pigigupk", Gûb sânganêniarpunga Kilangmut pijomâruma.
2. Jêsusemullo okpertor Erkartuivingmut pingilar; Taipsomunga okperpunga, Ajornikka pêrpait ôma.

3. Gûdib sânganut piguma Kiksarniænginivunga; Ubvartaungmat ômatiga, Pitlaræksaujungnaipunga.

4. Okperpunga Gûb Erningat,

Aungminullo tokкominut, Piuligamga tâktomit, Ajortunillo tamainit

5. Okperpunga Jêsub aunga, Nâmagijaulermat mânna, Gûb sângane: pêjarmagit Illûnaita ajornivut

6. Taimaimait tâpsoma aunga,

Mannigortiksirivara Inônimue tokkumnelo Tættigênaromavara.

7. Nâlagak! nakudlarpotit, Pillua tokkolauravit; Aymivut pêjarlugit, Kuviagijaukovluta.

8. Jêsuse nakudlarpotit Niuname inûlauravit, Illûncitalo inuit Pissiarigangne aungunt. 
9. Tikkikupko Jêsusiga, Imâk oxarniarpunga : Ajutsainarama illa, Aut pivlugo itterlanga!

10. Taimagle inôtillunga! Tamaita innurattikka Âniarnik sorairnanga Okautênaromavakka.

\section{Mel. 221.}

Sei fröhlich im Herren. 282.

1. Kuviasulerit tarnîk Nâlekame,

Annorâtârsimajotit!

Piulijivit amuadlarpâtit Tâktomit, idluarnermut; Ajornerit pêjarpait aungminut

Pekarkonnago akronaijomik;

Kanoêtitsijut, ipektotitsijut,

Mattulauкpait illa, pinnarnadlartomut,

2. Pivianadlarmêk pikka soraingitut,

Piulijima Kaitangit;

Assiolauktunnik Kaitsivigivânga

Tokkominut aungminullo. Okpernikut mânna tiguvakka,

Illerasungnanga, pigigapkit;
Pivlunga nellâgut piniulaungmagit

Pivlunga Kejungme nivingadlalaungmat.

\section{Mel. 16.}

Sünder bin ich, ja das weiss ich 284.

*1. Ingergarma Jêsusimnik

Ômisukteovunga;

Illakka piulijimnik

Okautjaukattarlanga!

2. Sunamullônêt nuname Idluarnimnullônêt Piulijaujungnangilanga Mannigortaunangalo.

3. Jêsub saimaningat kêta Piuliklerningallo. Ajortoerutivânga Piloriktilungalo.

\section{Mel. 58.}

Was uns mit Frieden und Trost erfüllt. 286.

1. Ullapirsaumik tarniptingnut

IllumertaulerKovlutale,

Piulijaunivut

Jêsub aunganut

Nagvâræksarivavut kaut tamât

Illuptingne. 
2. Jêsub tunnergutauninganik

Omamut tættekatsainartok,

Tunganilijauvoк

Kairtomaringmut,

Sillaksoak nungulerpallônêt,

Pêngitomut.

3. Assianigle tættekartoK Igloliorpok siorkanut, Piulijaungilak
Kellaktiminit,

Gûb saimarsauta akkekangitor

Nachogingmago.

4. Gûb nâpkigijomatsængilait

Inuit kaijomalungitut, Gûb saugârsunganut, Tokkolaurtomut

Ajornivut pivlugit KePiuliluta.

\section{ÔMAMUT ILLUITOMUT TUNNINERMIK JÊSUSEMUT.}

\section{Mel. 376.}

Gieb mir Dein Herz. 288.

1. Jesusima imâk окаupânga:

"Ômattît uvamnut tunnigungne,

„Perkojakka nâletsainarungne,

"Tessioromavagit

"Mâne inôtillutit."

2. Perkojanga uvaptingnut tagva,

Kuviasutsainaromagupta; Pilorikpulle taimailiortut, Perkojanik nâlektut Piksarsitsainarm 7 t $a$.

\section{2\%2. Mel. 82.}

Meine Seele, willt du ruh'n. 289.

1. Merngoêromaguvit

Ullapitaunæk tarnîk;

Piulijaujomaguvit,

Uvingub issumanginit:

Jêsuse ungagiuk,

Tagva taimailârpotit.

2. Ungajub tapsominga Angnerpâk erdligivâ, Naglingningallo kissime

Illangiutitipâtigut

Piloringnadlartunik,

Mânelo tâkpanelo. 


\section{Mel. 37.}

Das ist der grosse $Z_{\text {weck. }} 290$.

Pijariakarnerpâk

Torâræksanit:

Tun: idlarkovluta

Illûnaptingnik

Piulijiptingnut,

Opigosumut

Ungagivlugolo

Ômatiptingnut.

\section{Mel. 376}

Nun nimm mein Herz 291.

1. Ômatiga, tamaitalo pikka Pikit nægliktara Jêsusigia! Tin.igalo tarnigalo illa Illingnut perkovakki, Aungnullo âkilikik.

2. Igvit Jêsus pituarivagit; Omatimne-itsainalerittôk Gûdigale tungavigivagit; Kappiasukkumalo Tættigijomavagit.

\section{2\%5. Mel. 106.}

Nimm hin von mir, was du. 293.

1. Ômatiga pijomatyanmue Nagliktor Piulijiga!

Piuk! piniutiliurangne Aktorsarmaridlarlutit; Tagva illingnut kaipara Igvit perkutigigangne.
๘. Ômatiga assingnut illa Tumnijæksaringilara, Tunnidlalauravit pivlunga Unganarnerpângovotit, Kissivit perkutigivat Assingnut pijuksaungimat.

\section{Mel. 14.}

Mein Gott, das Herz ich bringe Dir. 294.

1. Ômatimnik Kaitsivunga Illingnut Gûdiga! Ikpigitinamga igvit, Tâmna tunnilugo.

2. A tâtak tâmnale piuk, Nachoginagolo, Sângue ajoktoliksôjok, Lllalliolauruk.

3. Illa ivsormartorsôjok, Mamaiiorsôjorlo, Kuksilaitok, tækkorngart: $\mathrm{K}$

Idluarijarnut.

4. Sângne Kakkialerpunga, Piuserijanmik, Mânna ajorniâlukka Ikpigillaraplit.

5. Gûde sittijuksônera Akritilaungmink. Idluitor issumaga l'êrle naglingnermut. 
6. Jêsuse taimak piginga, Ubvarlunga aungnut, Tokkogavillo pivlunga, Inuillo pivlugit.

7. Okpernikitub tarnima Tiguliuk aggut, Nekkoksiuk, ajornikka Pêjarangne aungnut.

8. Idluarsitilaungminga

Piuliklernernut, Ajornikka pêjarangne Pitlautiksakkalo.

9. Gûd Anernek, uvangatauk

Innigilaungminga, Jêsusib naglingningallo Kibliuk tarniga.

10. Kaumanerpît kaumarlinga;

Næglingnivîllo Gûd, Kiblidlarlinga, tâksoak Pêrlugo uvamnit.

11. Gûde ômattiga piuk, Nâlekvigilitit, Innigitsainadlarmiuk Nâksaungitomullo.

12. Issumangnik âkilauruk Idluarijarnut, Perkutaugama illingnut, Pinanga assingnut.
13. Sillab pingit allarlugit Nâlegungnaipakka; Jêsus nâlegomagapkit, Piuk ômatiga.

\section{9\%. Mel. 79.}

Herr Jesu Christ mein Leben. 298.

1. Nâlegar Jêsusiga!

Inôgutigijara;

Pigiluarminga;

Pivlunga tokrogavit,

Uvang: kaikogamga

Piginga Gûdiga igvit.

2. Issumaksarsiornanga Assingnik pijomârtut Pitjutigællugit;

Aggangnut Jêsusiga Tessiortaujomavunga, Tættigidsainadlarapkit.

\section{Mel. 70.}

Dem blut'gen Lamme. 299.

1. Aulik Nâlegar, pivlunga Kejungme Aunâdlarlutit, tokkotaulauktotit, Aniâlauktotit Nâlegak Ômattît sêngmago ajornima.

2. Illûnarmale, uvlome nutâmik 
Tunnidlarpunga illale illingnut,

Issumangnik attulaungminga!

Idluinimnit ubvalaungminga!

3. Piulijiga idluilutakka

Kaujimavattit, sangêdlaramalo;

Tamanna pivlugo tuavi

Ajornimnit inûlilaungminga!

4. Pêjarlugillo, aungnut kovijomut, ·

Illa pivlunga kovimadlalaungmat;

Uvlullo tamaita tarniga Idluarsaruk, pairilugolo.

5. Ajudlarpunga, nellungilarmale;

Naglikparmalo Jêsuse Kristuse,

Taimaimalle kujagivagit Inôtillunga nakorivagit.

\section{Mel. 14.}

Du ew'ges Liebeswesen Du. 301.

1. Nâksaungitomut nagliktor

Jêsus nertorlagit!

Kaut tamât pilligaptigut Ullapirsautingnik.
2. Timivullo tarnivullo, Inôserlo pikit Âkilaukittôk illingnik Nertordlerkovluta.

3. Idluaringitatit Gûd

Pêkit uvaptingnit, Ikkajortigullo, sumik Ivlerserkonnata.

4. Ajornivullo tamaita Kaujigangne pêkit, Amuatsainartigullo Tamainit illingnut.

\section{Mel. 183.}

Da ist mein Herz und meine Seele. 302.

1. Ômatiga tagga kaipara! Inniksautiuk illingnut, Anernekut kajangertitsuk Kuviasuktilugolo;

Naglingnivit soraingitub Taipsomane uvaptingnut Naglingnivit soraingitub Ômatiga innigiliuk!

2. Illumne nâmagingitattit Tamaita asserolaukit! Idluinerub illûnapkut Illainapkullônêt mâne Kallaklunga kivgarikpanga,

Adsigêkpor Gûde sângne, KivgaujungnaitisimajoK Kissiat idluarigangne

3. Illale, tagga illûnara! Nutâmik angidlarpagit: 
Nagligitsainaromavagit Illûnapkut pigilaunga! Illanâra attît tamêne Kikkiæktortaunillotauk Tækkuksautsainarlik raumne,

Naglingnermut taimaitingatôk!

\section{Mel. 37.}

Herr Jesu, nimm mich hin. 306. 1. Nâlegar Jêsuse Illingnêlanga
Næglingnît kissiat

'Tættigivara.

Inôjomagama

Sokkosernanga

Kuviagijarnut,

Taimaitiminga.

2. Kennuvigivagit

Mânna ômatiga

Pitsartutilauruk

Aukserlugolo.

Tarniga pivlugo

Tuksiarapkit,

Timigatauk aungnut

Inûlingmiuk.

\section{KRISTUSEMIK TARNIB ILLAKAROMANINGANIK.}

282. Mel. 151.

Kanok pinniarlunga Illalliorlutit?

Tarnima pinnarivâtit

Ungagigamitit, Attêle Jêsusiga!

Omatekulluga Aungnut serparsarlugo, Âkilaungmiuglo.

\section{Mel. 159.}

Das einige Notwendige. 308. Attauser pijarialik: Kristus pigilugo,
Tarne timelo tamarmik Mâne pitilugik;

Taimak inôsek nuname Tammarsuêrtok, sumulle Aksârnigausuêrluta, Agganginit illa.

\section{3a, Mel. 185.}

Eines wünsch ich mir vor allem andern. 309.

1. Attautsemik tussuluarpunga

Tamaitarsoarnit illa, Keaviptingne tamâne tagva 
Pilorigajarpunga:

„Takkonârlugo sokkosernanga

„Angut âniadlartor pivlunga,

"Aungmik kidjijârlune, "Toккomut pijauvlune!"

2. Ijîngma sângangnêtsainalaurle

Saugârsuk kennuêtor, Piulijiga aunâdlarlune Kejungme nivingajok, Tarnikulluga puigornago, Illa immerûtigidlarlugo; Pivlungatauk kejungme Nâmaksivôlaurame.

3. Puigortaililago nâpkinît, Ama pijæksaunera!

Kennuêvigidlarparma igvit Tâksoarmêlaurama;

Saugak tammartok kennênarlugo.

Nippingnik kammalerkârtinnago,

Akkitujomut illa

Pissiaridlarlunga.

4. Pigivarma! Amenêrvi-
ginga! Jêsuse, pigivagit! Nênilauruk ômatimnut tagva

P đivianardlartor attît! Aiparijomavlutit tamaine
Tokкomut inôvlunga illingne

Angervigidlarpagit;

Sôngotitsijôgavit.

\section{Mel. 146.}

Den meine Seele liebt. 312.

1. Erdligidlartara

Nellekangitoksôngmat,

Nellàgut tâpsoma

Assingit sunaungilat,

Illanârijima

Najudlaraminga,

Kiksad arneralo

Ômatimnit pêrpâ.

2 Tagva nagliktiga

Ajungitok tamainit.

Pijakka ômangat

Kujanadlarput illa,

Missigivarale,

Mâne najorapko,

Kingorngagulletaur

Tækkolermigupko.

\section{Mel. 15.}

Ich öffne Dir Herz, Seel' und Sinn. 314.

1. Ômatiga tarnigalo Angmarpâkka illingnut Kajungervigidlarapkit Illalliorlagittôk!

2. Kommunionekartinga! Kittorngait sangêdlartoK 
Ônarsititaukovlugo

Nagligosudlarnermut.

3. Saimanerub anerninga

Illilertutsainanga!

Arvertartitsainangalo.

Senniagôrkonnanga:

4. Ômatiga pimariguk,

Pitsartutilugolo!

Tagva piloridlarpunga

Jêsuse tækkogupko.

\section{Mel. 161.}

König Jesu, den wir lieben. 316.

Nâlagak nagligijavut

Mikkiluartomigle;

Issumavut aulatikit

Kellaklugit illingnut;

Kajungerti-laungmittigut

Kittorngaumarerkovluta

KattimajoKotingnulle.

\section{8\%. Mel 39.}

Gesalbter Heiland. 317.

1. Piulijivut saimarsaijungortotit!

Illanâkarnernut nellipsalauktigut

Pairksinernut mânna pijomârtomelo

Nertornadlartomik serngnigilauktigut.
2. Gûb sauganga illatit najutsainakit

Johannesib tækkolaungningatut sorlo,

Saimarsainit perkolauruk, scrairane

Uvinekattingne-itsainarKovlugo.

3. Ômatiga tækkotsainarle ikkingnik,

Idluarnermelo aiparitsainaruk;

Ullapirsaut anginerpauj oK tamainit

Uvamnêtsainarle soraijuitomik.

\section{Mel. 4.}

Eins bitt' ich vom Herrn. 320 .

1. Nâlegak sângne

Tuksiutiga :

Sokkosernanga

Illagêngnernêtsainaromavlunga.

2. Killanârnera

Taimaitsainarle:

Piulijiga

Kuviasuktitsainaromavlugo.

3. Ungagivagit;

Naglingnerale

Suglugigapko, 
Uvamnik nâmaksijungnangilanga.

4. Johannesetut

Illingnêlanga,

Killanârama

Aiparitsainaromagapkit mâne.

\section{Mel. 58.}

Ach mein Herr Jesu, Dein

Nahesein. 322.

1. Ahâgle Nâlegar Jêsuse! Najornît Kaitsivok ômamut,

Piloringnartomik

Ullapirsaumik;

Timetauk aglât kuviasumut

Nertordlermat.

2. Killanâritsainaruptigit

Illa, missigijomavlutit,

Kuviasukpogut;

Sorairatalo

Kujagitsainadlarpaptigille Angijomik.

3. KêmititsainartoK illingnik Unnuallo uvlut nâvlugit, PilorikpoK tâmna

Nâksaungitomut.

Timingat, tarningat nertorpâtit

Naglingnermut.
4. Nâpkigællutalo saimavok, Ajorniptingniglo pêjaijoK Mannigorpâtigut

Inûliluta,

Kajungilârnermullo tarnivut

Saipaksarpait.

5. Piuliklernernik tamainik

Illangiutitênartigut,

Taimaititigullo,

Kappiasumut

Unganermullo illingnû̂nak

Ârॅ̄rkovluta.

6. Kugviorangapta, mânnakut

MannigortiguttôK toKKungnut;

Ikkitit tamaita

AunârtoKsôjut

Kaut tamat tautuKôtsainarlavut

Tarniptigut.

7. Nutakkatun-ilertittigut, Tamanna pijominadlarpoK,

Ikkivit aunganut

Serparsartigut,

Taimailiorvigitsainartigut Kaut tamâle.

8. Tagva kuviasudlarpogut Angerarkârata kilangmut. 
Unêt sulle mânna

Kugviorupta,

Ômatiptale najorangattit

NekkoKpogut.

9. Tigusârpaptigut aggangnut,

Tessiorlutalo nellâgut;

Tagva erkarlugo,

Opigukpogut,

Kujalinermullo ijivuttauk

Kugviorput.

\section{Mel. 230.}

Sei Du mir nur immer freundlich. 324.

Illannâritsainarminga

Oкumaitokalerangama

Kugviorangamalônêt,

Missigitilaungmingalo

Mannigortaunimnik illingnut

Inôtillunga nuname;

Nâlegar Jêsuse

Ungatilaungatôk

Illingnulle

Sorrusertut nagliktotut Ânânaminik ungamut.

\section{Mel. 36.}

Ach Delnes Gnadenangesichtes. 325.

1. Kênavit kaumasingat kaumarlinga

Illuvermut illijaukârtinnanga;
Illannâkarnernik naglingnerniglo

Ikpigilanga!

2. Timigalo tarniga tokkoville

Uvlut tamaita nekkoksiligigle,

Najornivit salutekattarlinga

Illingnêlunga.

3. Illingnik kivgartorkojomagumga,

Perkojattit malligomadlarpakka,

Najornernik tukkisitsainarlanga,

Nagligigamga.

\section{Mel. 185.}

Dein Verdienst und Deine liebe Nähe. 326.

Piuliklernîllo najornîllo

Nekkorsitigilakka;

Gûb Atâtapta naglingningallo

Illagitsainarlinga,

Anânatullo Gûb Anerningat

Pairilinga, nâmakserkovlunga,

NekkoKsijutiksakka

Nellonangilat tagra. 
293. Mel. 124.

$\mathrm{O}$ was ist für süsse Ruh', 327.

1. Merngoêrsidlarpunga Jesusimne,

Iksivatsainaruma ittiggangine;

Illa angmartipara ômatiga

Oкausinginulle.

2. Mânepunga Gûdiga!

Tussartinga

Pijarialiksoak

Kennerlugo

Malliktitsainarminga

Perkojarnik,

Nertortaukovlutit.

\section{Mel. 218.}

Erhalt' mir Deinen theuren

Frieden. *Anh. 90.

Ullapirsautingnik Jêsuse

Tunnitsivigitsainanga!

Sangênimne pitsartutinga,

Okumaitut pêrsârlugit!

ErKomanimne sinning-

nimne

Siorniornerilauktavit

Siarklerungnaitilinga!

Ânianitillo tamaita

Kuviasûtigênarlakka

Illingnut tikkerKârnanga

\section{Mel. 217.}

Im Umgang mit dem Schmerzensmann. *Anh. 91.

Angut ânialik mâne Nellâgut aipariguptigo, Uvlut kuvianadlarput; Ômatîk apperkutigiuk Tamât, uvlok kaulerangat, Seккinerlo nippilerpat, Najuganêtsainarkovlunga, Sullinimne rikkarnimnelo, Ikkinginut âkiklunga, Kaumanermut aikârnanga.

\section{Mel. 159.}

Erhalt' mir, Herr, das Glaubenslicht. 266 .

Okpernerub raumasinga, Tækkoneк tokkungnik, TætteKarnerlo illingnik, Piulijiptingnik, Aulajuitikik illumne, Naglingnerlo, naglingnernut

ÔnartisimatsainartoK Pigitsainarlago.

\section{9\%. Mel. 244.}

Wir blicken nach der Höhe. 329.

Ârlorpogut takpaunga, Jêsub nagliktipta

Tækkonâlermattigut;

Ijiptale tâmna 
Tautugungnangilât,

Tarnipta missigivât,

Najorningalo mâne

Kuviâgidlarpat.

\section{Mel. 146.}

Mein unschätzbares Gut. 330.

1. Nertornak tamainit Mattomanêtillunga, Attolerangapkik Jêsub timâlo aunga; Tâpkoale mâne Komunionikut Ôklugik kaut tamât Sulle ajornarmat;

2. Tagva nanêkuma, Uvlamit ninuamut Piuliklerninga Erkarasuarpara; Okpernerma mânna Inôtigidlarpâ, Tækkovara sorlo Aiparidlarapko.

3. Angut ânialik Kemergojomavara, Inûlervianit

Kejungmut tikkilunga; Tautukôrangapko Aјокеrtuijoк OKâl tillugo, Illuga ônarpok.

4. Piniarningille Pitsartutigivakka;
Pijangit nuname Pilloreutigivakka. Mannigortauvunga Marîa Magdalena Erkarluarapko, Nagligidlarmago.

5. Arnak keadlartoк, Keajungnaitipâle, Erninga tоккојок Tâpsoma ômartipâ. Lazarus toккојок Illuvermêlaungmat, Naglimut nangmineK Kugviolaurivoк.

6 Tarnima Jêsuse, Erkarpa serrinermut, Illinniartime Ittiggangit ubvarmagit, Johanneselotauk Nâletsiartorle, Tautukôrparataur Êgarvigijinga.

7. Kommunioneme Aitortormagit ôma Illinniartine, Timiminik aungminik, Tautukôrmigama Tamaita pivlunga, Pitsartutimmagit Pilloreutikka. 
299. Mel. 14.

Lass unsere Seele Schritt vor Schritt. 331.

1. Tarnipta Jêsuse tarnît Najutsainarlitsuk Ômativut serparsakit Aungnut kovijomut.
2. Piulilaungikuptigut Aniadlarnernut, Assionajalaukpogut Issokangitomut.

3. Aungnut piuligaptigut Kittorngakotivît Pilloreutigilutit Tungavigivâtit.

\section{ULLAPIRSAUMIK KUVIASUNGNERMIGLO ANER-} NERME AJUNGINERME.

\section{Mel. 167.}

Meine Seel' komm' in die Wunden. *459.

Tarnîk Jêsub ikkinginut Kait merngoêrsernermut, Nagvârvingmut ullapirsaumik,

Tarnîk tagvungalaurit!

Issumalingasuêrit

Merngoêrserlutillo

Tagvane aikojauvingne Aggangine sângane.

\section{Mel. $115 \mathrm{~b}$.}

Wol dem, der Heil und Frieden hat gefunden. 334.

1. Pilorikpor illa, ullapirsaumik

NagvârsimajoK, Atâtamullo

Agviartaunane najûtsungnartok,
Ominautêjarsimajoк Jêsub Ikkinginut; tâmna illa Alliajuteкаrpor Nungusuitomik.

2. Gûb saugangata piuliklerninga

Kaitsivor ullapirsaumik, ôma

Assingane nagvârtautsængitomik;

Pigigupkolo, pekadlarpunga

Pijariakadlarnimnik; Kellujauкovlunga Erkartortaunermit.

\section{Mel. 221.}

So ruht mein Mut in Jesu Blut und Wunden. 346.

Jêsusib aunganelo ikkinginelo 
Illa merngoêrserpunga, Illûnarma ômunga illigamalo

Nekkorsijauvunga illa; Ikkingit innigênalerapkit, Tarnimalo pituaringmagit:

Naglingninganiglo tukkisilerama

Inôsimne tagva allaromaerpara.

\section{Mel. 37.}

Volk Gott's die Freud' am Herrn. 336.

Inukutaujose

Gûmut, Jêsumik

Kuviasungnise

Nukkigissiuk ;

Mânna najorpâse

Nagligijipse,

Ullapirsauminik

Tunnidlarpâse.

\section{Mel. 184 a.}

Zeuch hin mein Geist. 335.

1. Annernîk Jêsub ikkinginut

Aunganullo aisâlaurit,

Immerlutillo tâpsomangat,

Nâmaksititaukovlutit.

Aisâlaurit puailavingmut Ômunga, kôtsainartomut Ullapirsaumik mamaktomik

Tarninut immeruktunut.
2. Kissivit Kuviagilagit, Sakkervigênalaungminga, Kiksautigijakka pêlaukit Kappiasungnêjarlunga; Igvit naglingniktorsôgavit Kittorngattit najorpattit; Kennertut nagvârungnarpâtit

Katsungaivigigunitit.

\section{Mel. 228.}

Friede, ach Friede. 337.

1. Gûdib ullapirsautinga Atâtamit pijor illa : Kristokut Anernerme, Ajortut ômatinginut Pititsijor inôtsemik Kuviasungnermiglo: Ullapirsaut tâmna, illa Illingavoк Okpertunu Jêsusemik ungajunut.

2. Ullapirsaumik sakkêjor Âniadlartuksaulaukpor Gûd ullaproserlugo: Tarnîk tamanna kamagiuk

Kemergotsainarlugillo Jêsup piniarningit; Ullapirsaumik aungminut Kaitsilerpok, pêjaijomik, OKumaitunik tamainik. 
3. Taimak kamagilaungmattit

Perkutaujuksaugivotit

Tâpsomunga tamâne:

Piuliklernermik illa

Sakkertitsingmet pivluta

Sorainiængitomik.

Taimaimat kamatsiarit

Illûnakut, tâpsomunga

Perkutaumarerkovlutit.

4. Jêsub ajokertûsingit

Ivlerilugit mallikit

Sennjagôrnagillo:

Innerterutigijangit

Ulloreagitsainakit,

Tagva pilorikpotit,

Piloridlarmatta tarnit

Mâne taimak tunnidlartut

Ingmingnik Piulijimingnut.

\section{5 a. Mel. 7.}

Alle Deine Gaben. 338.

1. Kaitatit illûnatik

Nakkoksinadlarput,

Jêsusîk, angijomik

Kuviasûtigivavut.

2. Igville tamainit

Pinerpângovatit,

Illiktut sumiglônêt

Nunamêtokangilak.

3. Ittervigilaur $\because k$

Nangminer ômatiga,
Illumertaukovlugo

Inerkonadlarnernik.

\section{Mel. 114.}

Mein Salomo. 340.

1. Uvamnigle pairksigavit Gûdiga!

Pêrpattit tarnib okumainingit

Illûnaita ; torârvigigupkit, Anernerublo ullapirsarmanga

Nekkoksivunga saimarsainernut

Lrksijungnaitinamga illupkut.

2. Aggangne Gûd, innilangalerpunga,

Nekkoksilunga nâkaungitomut,

Igvit ullapirsautigioapkit; Nâpkininginit inôgutigigapko

Illa; Gûde pitudrigapkit

Nâmaksivunga missigivlutit.

\section{Mel. 95.}

Wol dem, der in Jesu Armen. 342.

1 Illa tâmua pilorıkpor, Jêsub nâpkiningninganut Ullapirsatutisijor ;

Illinganerue timaine.

- Illa torkortautsiarmatḰuviasutsinimarpok. 


\section{Anernerme ajunginerme.}

2. Illa tarne serridlarpok! Tunnigupta saugârsungmut

Kivgartoromavlugo;

Tagva ômat ikkingine Inôtsainarpok tamâne Saimarsautekarlune.

\section{Mel. 218.}

Wie wol ist mir, o Freund. 344.

1. Kưiasûtigitsainarpagit Tarnimnik naglingniktotit! Nênertaunadlartovingmille

Ômatingnut aisârpunga Kiksarnadlartut pêrput tagva

Ômativîllo naglingninga Missigigapko illumne, Tagva sorlo kilangmêpunga.

Kina Kuviasutsængilâk Illingnik piksarsigune?

2. Ajortut nênidlalerppanga Pikpangalo ôminartut, Perкojallo erkartorppanga,

Tagva ârlorpunga kongmut,

Ikkitillo aivigivakka, Tâpkoa torârvigivakka, Tagva erksijungnaipunga, Pitlaræksaugalloartunga; Piulijigigapkit illa Naglingnivit torkorpânga.
3. Kanorlônêt tessiorumga, Égarvigijomavagit, Pingnik nerrititsainarparma

Immilungalo ikkingnit. Tessiortigijomavagit Nagligosudlartotôgavit, Najorumga nâmaksivunga. Pivlutittauk serridlarlunga

Kaikogumga angerarpunga

Kuvianartovingmulle.

\section{Mel. 164.}

Wie wol ist mir, wenn ich an Dich. 345 .

1. Illa piloridlarpunga, Igvit erkarangapkit, Tarnigalo Jêsusiga Ikkingnut pitikupko! Jêsus illingnênak Kuviasukpunga, Tarniga innilangakpat Ittikkaville sângine.

2. Illa piloridlarpunga, Kêmitivigigupkit, Anerneralo illingnut Sungiutilerupko! Taimaitilaungator Tamâne nuname KuviasûteKarkonnanga Assingnik, Piulijiga. 
146 Ullapirsaumik kuviasungnermiglo.

\section{Mel. 189.}

Seitdem das Lamm am rauhen Kreuz. 348.

Saugârsuk kejungme ânialaungmat,

Ullapirsaumiglo Kaitsimingmat,

Okumaitut illûnatik oKiput,

Ullapirsautingat pingmattigut.

Jesub tokkunga

Anianingillo

Kaitsitsainarput

Ullapirsaumik.

\section{Mel. 155.}

Sünde und der Sünde Sold. 349.

1. Jêsusib rennunikka

Nachogijomangimagit,

Ajornerit

Kivgarijungnaipânga:

Saimanek

Tâpsomangat pigijara

Angnerpaukutigivara,

Illumne torkorlago!

2. Nâlegar nertorpagit,

Nukkerangitok uvanga,

Ajulertok,

Ullapirsalauramga;

Taimaktauk:

Gûdib inukotinginut

Illalliortilauramga

Piulijigapkillo.
312. Mel. 75.

Wenn man die Gnadenwahl. 350 .

1. Annerimanivut

Jêsub Ikkingine

Illa nagvâruptigo

Nekkorsijigivlugo;

Kuviagidlarpavut,

Nagliklugo omâmut.

2. Gûb sangane inuk Ajornerpângojok, Kakrialerunele, Ajungitsuginane, Naglimut nâplkigivâ, Kellarudsârlugolo.

3. Nâpkigijaunine

Erkaumatsainarpâ; NoKangilarlo tâmna Axiktauviksamine. Gûb ullapirsaininga Najortigitsainarpâ.

313. Mel. 151 i.

Geist Jesu, dir sei Ehre. ${ }^{*} 479$.

1. Jêsusib Anerninga

Opigilaptigit!

Ullapirsartaugapta

Ajokertuinernut.

Jêsusib tokkunganut

Piulijaugapta;

Okautigingmilavut.

Missigigaptigo. 
2. Inôsek ikkingine Tarnib serrigivâ, Opigomut ômame Kugviudlarluta;

Saimanek ikkinginit

Ômamigijavut,

Illûnatik inuit

Kanoktôk ôglitsuk!

\section{Mel. 82.}

Jesus unser Hirt ist treu. *Anh. 106.

1. Pairijipta Jêsusib

Nertornartotôninga

Kaut tamât tukkisivavut, Saugakotimitut sorlo Pairitsainarmattigut, Merngortoranelônêt.

2. Anne rijipta Jêsub Kaitsivigingmattigut Piksanik nâksaungitunik, Kuviasûtiksaptingnik Ullapirsarnartunik, Kujalivigilavut!

\section{Mel. 205.}

Unser Loos ist schön und gross. *Anh. 111.

Anneri-jaumanivut Jêsumut, opinadlarpor. Ingminik tunnilune, Illagijomavâtigut; Allatsængimattigut Mannigortigivavut;
Aipariguptigolo,

Pilorikpogut illa.

\section{Mel. 230.}

0 welch unschätzbarer Segen. *Anh. 112.

Jêsusib inôninganit

Saimanernik angidlartunik

Tarniptingnut timiptingnut

Pitsainalerpogut mânna. IssumangnarungnaineksijoK

Ajortunit ikkingine, Tâmna pekalerpoк Saipakutiksamik;

Ômatinga

Oкilerpok

Okpernermut

Piulijipta tokkunganut.

\section{1\%. Mel. 185.}

Jesu, treuer Heiland, sprich. *Anh. 122.

Jêsusiga ullapirsautingnik Pillitsainalaungminga, Ômatima uvlune tamaine Taimaktauk unnuane, Illanâkarnît serrigiliuk Nekkoksijaujutiksamik mâne

Ajoksalerkonnago

Tiglerungnaikârane. 
318, Mel. 141.

Meine Wünsche schliessen.

*Anh. 120.

Tussugidlartakka

Kennunermut kattivut:
Jêsus aunârnernut

Perkutigilaungminga!

Tagva inônimne

Pilorikpunga

Ullapirsaumiglo

Ajoksarnanga.

\section{GÛDIB KITTORNGANGITA PILORINGNINGINIK.}

\section{Mel. 136.}

Wie wol ist mir, dass ich. 353.

1. Kuvianak

Jêsusib ikkiminut

Aungminullo ajornikka pêrmagit ;

Kennertara kajungilârnermut

Kristusib tokkungane nagvârpara,

Tokkosuitorlo inôgutigigapko

Tokкosuêrtitauvunga,

Kuvianak!

2. Pairijima

Tarniga nerritipâ

Kakkôjamik ômanadlartomigle

Immipânga, illale aungminik.

Kiksarnartor sivôrajungnaipara

Jêsusib Kristusible najutsainarmanga,

Ajoksarkonginivânga

Pairijima.
3. Kujalimut

Nerrivlunga immerpunga

Jêsub piminut nekkorsitimmanga,

Kaut tamâle, Kannimakonnanga,

Issumajârtuksaungilanga mânna,

Tâpsoma piksamnik tunnitsivigingmanga,

Nerrivlunga immerpunga, Kujalimut.

4. Inôvunga

TokKosuêrtinamga,

Tattamnartullinitit nertorpakka,

Okperigapkit erkartornanga,

Tokkolo pêjalerangne pivlunga,

Uvangatauk inôtsemut pititauvunga

Tokkosuêrtitauvlunga, Inôvunga. 
3:0. Mel. 218.

Jehova ist mein Hirt und Hüter. 354.

1. Jehôva pairijigigapko Ajoksarniangilanga, Piminik piksakartimmanga

Nekkoksititsainarlunga; Aitipânga puailavingmut, Tagvane uvaptut sangêtut Ajortullo naipipakka.

Nukkingêrutilerangama, Pitsartutinivâ tarniga, Pitilunga inôtsemik.

2. Illa tessiorluarpânga, Mallituinarparale; Kappiaktoadlarumale Tâktokut pigumalônêt, Sangêdlunga, kiksadlaruma

Siorniortuksaungilanga. Igvit najutsainaramga, Kaumaksartigitsainarpagit

Tessiortigitsainarlutit, Igvit êgarvigivagit.

*3. Hallêlujârvigidlarlagit Saugârsuk pairksijôjotit; Kannekarlanga tausendinik

Nertutsiarungnarlutit !

Tunnergutille pijomanagit umamik killanâdlarpotit Illingnut perkutaujomik.
Taimaitokartilaunga; tagva

Ikpiginarmêk: „Pairijima "Pigivânga, pigivara.“

\section{Mel. 115b.}

Wie herrlich ist's ein Schäflein. . 356.

1. KittorngauneK Kristusemut ânanâk!

Idluarnerpângongmat nuname,

Illinganek tamana angnerpauvok

Malliklugo soraijuitomik; Kia Kaitsungnangitangit Piksarijungnarpait Gûb kittorngangita.

2. Inôluarvekaromajub tâmna

Pairksijok najugarilerliuk:

Tâpsomane piksakadlarрок tagva,

Sivorngagut piksakalaungitok,

Mâne ajoksatsængilak,

Nâlegar tamainut Pairijigingmago.

\section{Mel. 97.}

Mein alles, mehr als alle Welt. 358.

1. Tamainit piksak, Jêsuse! Pituarijagit mâne, 
Illannâk nâksaungitomut, Tokkotaujok aunârlune. Nekkoksivunga tessiorumga,

Torkorlungalo saputigamga.

2. Ajungitamnut tamainut Sumik pijungnangikuma, Sillainera sangênera Ikpigituinarupkik, Tagva, ajugakangitôjotit Tamaine ikkajorungnarparma.

3. Tamanêkojomagamga, Illingnik kivgartorlunga, Ajaupiarilaulagit!

Uvluksakka. kittikangne, Arvertarnera aulatilauruk, Idluarijarnut perkovlunga.

\section{Mel. 166.}

Die Gnad' ist unaussprechlih gross. 359.

Saimanerub angininga Okautaujungnangilak; Ajortut nachonartullo Idluarsititauningit Innorpavut, Jêsusible Aungminut ajorningit Pêrlugit ullapirsarpait Gûmut, âkilugillo.

\section{Mel. 155.}

Christen sind ein göttlich Volk 361.

1. Okpertut pilorikput, Gûmit erniangomavut, Ungagivât, Ônartisimavullo

Tâpsoma Anerninganut, Jêsublo Aunga pinnarsitigivât Tautukôtsainarpâllo.

2. Jêsus pigerkârnago, Ivlerijungnaikârata, Ingerkârata, Ôma ittiggangine, Ungasid-larpogut saimarsainermit, Apromillo amitomit, Kaub uvlorianganit.

3. Illannâk amuartigut

Ômatingnut nagliktomut, Kajungersainit

Tessiortigilavut

Nunakut.

Perkutautsainarkovluta Illingnut, okperlutalo Takpaungarkârtinnata.

\section{Mel. 185.}

Sollt ich nicht von Lieb und Dank entglommen. 368.

*1. Kujalinermut naglingnermullo

Ônartuksaungilangâ? 
Kina pivlugo inungolaukâ Gûde? Illa pivlunga. Assimatauk piulijigivât, Ikpigidlarumale, uvanga Piulijaulaurama, Kuvianarnerpâk tagva.

2. Illanâra aipatuarigupko Sorlo kilangmêpunga, Imâk erkailerama, Jêsusib

Kittôrngarilermanga; Kiksartuksaujungnaipunga tagva, Ajornimnik sannikteriguma,

Jêsusib najorninga

Mannigortigivara.

3. Ômatimne ersaumalerapko,

Jêsus aklulerlune,

Uvaptitut avatekarlune Arvertarmat nuname; Tagvale timiga pairivara Sorlo Jêsus Kristusib timinga,

Pinasualerpunga Adsigijomavluga.

\section{Mel. $22 \mathrm{f}$.}

1. Nâlegarle Jêsusiga! Illingnut inûlerpunga, Aungnut pissiarigamga Pigitsainaromavlunga.

2. Mattomanêtillungalo Aut, tokkullo, ikkitillo Inôgutigidlarpakka, Piksaringmagit tarnima.

3. Kêta unêt najungikumga, Ajulertotun-ipunga : Taimaimalle nutakkatut Tessiunga âniarnut.

4. Anernerpîllo timiga Innigingmago, tarnima Kemmakpago, ômartitsuk Illingnullo ailaungmiuk.

\section{Mel. 151 i.}

Nelloprotidlarupta, Kuviasukpogut Missigilugo ôma Najudlarmattigut, Mannigudlarpâtigut Kugvêjalerluta; Kanoêtsainarpogut Jêsukangikupta. 


\section{JÊSUSE KRISTUSEME INÔTSEMIK NELLÂGÔR-}

\section{TOMIK.}

\section{Mel. 164.}

Jetzt ist die angenehme Zeit. 370.

1. Nelliutijok serrinar!

Kilak angmarsimangmat,

Illanâkamut Nâlegax

Sakkermallo ômame,

NâpkiningningatôK

Sakкertilauktanga,

Illûnata opigomut

Nertordlerutigilavut!

2. Sillaksoab illusingit,

Nachogimariklavut,

Mânelo ivsornainermik

Pinasuatsainarta!

Okpernek nanêkâ?

Ikkomamarika

Tarniptingne? Uiksaktôk

Taimak pagrealerlavut!

3. Tapserusijatsainarta

Makkitiptigut mâne!

Taimaglo ingergarnivut

Takpaunga nâlerlavut!

Merngoêrsernermut

Tagva itterpogut.

Kiksarnartullo soraiput,

Kuviasutsainarpogut.

\section{Mel. 173.}

Eins ist not! ach Herr dies Eine. 373.

1. Attauser pijarialik, Illitarilerlago!
Assingille illûnatik

Okumaititsingmatta,

Ômatib kappiasûtigivaktangit

Kuviasûtiksarsinane nellâgut,

Attauser tamanna pigile-rupko

Attautsemut tagva nekkoksivunga.

2. Tarne nagvâromagungne Kennernago inungne;

Kemmakit nunab pingille; Jêsume kennerlugo,

Ômane illûnatik illagêngmatta,

Sakkermattalo pitakaloringnerit;

Tagvane piloringneк nagvâræksaK

Attauser piulijiksatuara.

3. Sorlo Piulijiminut

Marîa kippilermat,

Ittiggangita sangænnut

Inginame nokartok,

Ômaminut siutiminut nâlekpâ

Tussaromainaramiuk okausinga ;

Jêsusib kissiat pijomajanga

Missigigamiuk, kuviagivâ; 
4. Taimaktaur kippilerlunga Jêsus ungagivagit;

Ômatimne sorainanga

Missigiluarlagit;

Inugasæksuit tunnulerikpattit

Uvanga malligomatsainadlarpagit,

OKauseraravit ômanartunik

Tamainik tarnima piksarijanginik.

5. Gûmut nellopkotijunga

Tættituarivagit,

Jêsuse pilorikpunga

Pivlunga auktogavit,

Kejungmêtillutit pêjaivi-

\section{givarma}

Pitlautiksamnigle tokKotigigamga;

Piuliklernerille okpernermut

Tættigivarale nâksaungitomut.

\section{Mel. 115.}

Wie selig ist's, nach Jesu Heil. 375.

Piloringnarmêk illa! Jêsusipta

Inôninga piuliklerninga, Kêdlarnermut kippivigidlarlugik,
Nipporsaratalo, illuptingne,

Ikpigidlarkârtinnata,

Ullapirsautinga

Pigidlaraptigo.

\section{Mel. 184 a.}

Dorten wird ein reines Herz. $* 503$.

1. Takpâne ômat ivsornaitor,

Inuit perkutinginit

Tamainit angnerpaujomârpok ;

Mâne ullapкuteкartok

Gûmut, takpâne merng-

oêromârpok

Ullapitaunane illa,

Pijomajanganiglo pijor

Takpâne erkiertorpoz.

2. Inuk! Jêsus kemergoleruk

Igsagæksaridlarangne, Tunnilerillo tâpsomunga; Nangmautinga tiguleruk, Malligvigitsainarlugolo, Tagva saimarsainerminut. Illigitsainarniarpâtit Nangiarnartokarangat.

\section{P32. Mel. 29.}

O Jesu, lass doch hier auf Erde $n_{\text {o. }}$ 377.

1. Nunamêtillunga Jêsuse

Illingnêtsainatilaungminga! 
Mâne ôgialerlugo,

Pâne najutsainartara.

2. Kanoktôrle piniarlanga Nertornartomik, âkiminga;

Angergalerkârtinnanga Nellagôtsainarkovlunga.

\section{Mel. 218.}

Ich will dich immer treuer lieben: 378.

1. Naglingnersarijomavagit Jêsuse nekkoksitinga; Apkosinernik attorlanga Merngoêrviksarsilunga, Nellekangitomik nuname Ânanaunerpângojomiglo, Kilangmut itterkongmanga.

Nâpkigigamga, ômatiga Perkutaukovlugo kaipara, Kajungertitsuk illingnut.

2. Inôsera tamât Jesuse, Tagva perkutaumarigle Illingnut tussugidlartamnut!

Illingnik missigitinga!

Nagliktara ômariktinga, Attaruilidlarkovlunga Merngoêrviksiorlunga Ômatingne sêksimajome. Pigilauruk pijomajara Taimaktauk pitsartunera.

\section{Mel. 230.}

Jesu, der, du uns erworben. 386.

1. Kejungme tokkolauravit, Piloriktuksangortipattit Illunaitale inuit:

Saukavit illitarivâtit,

Pairijimingniglo taivâtit, Saimausertaujomavlutik! Ullapirsaijotit

Saimaromavluta

Tussugavit,

Jesusivut

Saimartigut

Inukotigigaptigut.

2. Piloringniksat illû̀natik Tokkungnit Jêsuse pingmatta;

Tamâne inôtilluta Âritsainalaungmittigut İ̉luarijarnut, uvagut Ajungitoranginapta; Pitsartunernulle Ômartilerangne Ômativut, Ingergamik sangêdlartut, Tоккut inôtigilavut!

3. Inôsiptingne nuname Tamât, ômativut Jêsuse Illingnêtsainalaungmilit. Nagligênalauraptigut Tokkungnut piulivaptigut:

Taimak nagliktokangilak Assingnigle illa! 
Erdligivaptigit

Angijomik;

Ômativut ônartikit,

Ungagitsainarlaptigit!

\section{Mel. 164.}

O Lamm! das überwunden hat. 388.

1. Saugârsuk! sâlakalauktôk,

Sâlakarmarilaurit,

Pekarungnaerkârtinnago

Uvaptingne sunamik,
Akkerartortomik

Illa okausernut.

Adsiovalliatitigut

Illingnut Piulijivut.

2. Kellarudsâlaungmittigut

Nangminerijaptingnit,

Kuviagingitarnillo

Mâne inukotingne.

Piniarkonnata

Sunamigle illa

Nâmagijaulungitomik

Nâlegavut oKausernut.

MALLINGNINGNERMIK JÊSUSEMIK, MLLANGIUTINERMIGLO SIORNIORNINGANIK.

3อย์6. Mel. 90.

Mir nach, spricht Christus. 390.

1. Kristusemiut tamapse

Malliksingârpâsele,

Taimak ajugaukojipta

Pivâtigut: „Okokse!

"Sumut pijaugupselônêt

"Nâleksingale tamainik."

2. Nagliktok Nâlegak tâmna Mallitsainarlavulle, Kappiasugviptingnelo, Sivordlerigaptigo; Nellâgôngitor, kilangmut Pijuksaujungnaipor illa.
33\%. Mel. 68.

Jesu geh' voran. 392.

1. Sivorlilaurit Jêsuse igvit Inôtseme, uvagullo Malligomagivaptigit;

Tessiortigut

Illa kilangmut.

2. Okumaitokut

Pijuksaugupta.

Sokkosijuititigut,

Ogguarkonnata tagva;

Apkut illingnut

Mâne amingmat. 
ค7月8 Mel. 16.

Bleib bei Jesu, meine Seele. 393.

1. Tarnîk illa, Jêsuseme

Mâne itsainalaurit

Inôsek annajanarmat,

Udsertutsainalerit.

2. Pigijarnik sapkosinæk Okpernermut Gûdemut, Sunamullo utterkonæk; Sokkosijuilerit.

3. NippingajokaranetôK Omatingne sunamik, Kellaksortitsijunigle Ipperaisiarlutit.

4. Inôniarasuarit

Jêsusib tokkunganut, Sumut ivsornarserkonnak Tâpsomanêtsainarit.

\section{BD9. Mel. 146.}

Herr Jesu, führe mich. 395.

1. Jêsus tessiunga!

Inôtillunga mâne;

Illingnut pilunga

Uvamnik pinangale;

Uvamnik piguma

Tammarsâlerpunga,

Tessiorumgale

Sapputijauvunga.

2. Ajungitullinît

Pilerangne illumne,
Nâmaksitênarpat,

Illingnêtuarpunga;

Nâlegomavagit

Kiksarumalônêt

Serrigumalônêt

Nunamêtillunga.

\section{Mel. 16.}

$\mathrm{Du}$ allein must uns vollenden. 397 .

1. Jêsuse, mâne kissivit

Âritsainartigulle,

Tættituarigaptigit,

Tarne nagligigangne.

2. Illa akkitudlartomut

Pissiarigaptigut.

Kivgaukonnata ajortomut;

Tokkungnut âkitigut.

3. Toккungnut amuartigut,

Kikkiæktortaulerlit

Uvaptingnik agviartut;

Pâne nertorlaptigit.

\section{Mel. 164.}

Wie gross wird meine Freude sein. 400 .

Illâle, angidlartomik

Kuviasulârpunga,

Okpernerme Gûd illing-

nut

Nellâgôtsainaruma,

Nachogijaunermik, 
Kiksarnermiglônêt

Kamananga torârlutit,

Pâne nertoromârapkit.

\section{Mel. 155.}

O Du Seelenbräutigam. 401.

1. Tarninik pairksijotit Illingnik illitarksijut Taisijullo

TættiksaKartuksauvât Assingnik?

Aukak illa, pingortitar Sangêdlarpok, Nâlegaujok Tarnipta pijomavât.

2. Okpertut nunab pingit Pituarijæksaringilait. Kuviasumut, Imâgle perKojauvut: Ikkilik Jesusênar malliklugo: Aglât kappianartokut, Tâmna sivorlerivât.

\section{Mel. 26.}

Wir sind Dein Eigentum. 405.

1. Perkutigidlarpaptigut Jêsus! tarnekut timekullo, Pitlaræksaunajarpogullo Malligomangikuptigit.

2. Attannîk! kina illiktun Ômamik pijomajungnarka?
Kia âniatigilaukaûk?

Igvit tokkotigilaukpat.

3. Attannîk! pijomajarnik Tagva tarnimnut aglalaurit;

Sunamik pijomakonnanga Assingnik Piulijiga.

\section{4t Mel. 221.}

Wir ruhen in Jesu dem Felsen. 409. KairtoK Jêsuse saputsauvigivarut

Kiksarnartut pềrkârtinnagit, Issumagingmattigut Kaujimavavut,

Aklunivut nellungilâ;

Illa erKsuktuksaujungnarnivut,

Nâmaksitilugit pivâtigut, Uktortaunerilaurtavulle

Kângertut

Tamakkoa nertordlerutigivavut.

\section{Mel. 58.}

So zieht die Gemeine dem Lamme nach. 413.

1. Kappianadlartokut aglât Illagêt Jêsuse mallikpât; Tamanna pivlugo

Kiksartitaugunik 
158 Mallingningnermik Jêsusemik etc.

Kuviasudlarput nertorpallo

2. Inônata nangminiptingnik Jêsub aune kovilaungmago
Seningajolingme

Tоккolaungmallo:

Okpertut tâmna kissiat mânna

Mallitsiarpât.

\section{JESUSIB NAGLINGNINGANIK INUNGNIK.}

\section{Mel. 166.}

O Jesu, Herr der Herrlichkeit. 414.

1. Ânanaunermut NâlegaK Jêsuse! issuanit Imgertokalautsangilar Nâmaktomik illingnik Inûlitigut nutâmik Illuvullo kiblikit, Opigosumut naglingnît Imgerutigilavut.

2. Iksivagalloartotit

Mânna ânanauvingne; Naglingnît soккоsingilaK: Sorlo kejungmêdlutit Napaudsaugavit pivluta, Taimaitsainarivotit! Uvaptingnulle naglingnît Sorainiatsængimat.

\section{4\%. Mel. 208.}

Einer, ach nur einer. 415.

Nellekangitôjok

Naglingniktotôjor
Kujagivagit,

Jêsus pairigangne

Kennuvigijitit

Sângne Keajut;

Taimaimat nâpkinivit

Angininga tailugole

Ajornarpok illa.

\section{Mel. 168.}

Herr Jesu! Du hast unsern Jammer. *557.

1. Jêsuse tækkolaukpotit, Ogguarnarniptingnik, Illapsugosumut tagva Sappermarilaukpotit Kângervigijungnarluta, Omatit aumalilaukpor; Kanimasivut pivat Ailutillo tokkomut.

2. Naglingniojotit igvit! Kia taijungnarkauk Naglingnivit augininga? Ajornarpok tailugo. Nertortautsainalerille, 
Jêsusib naglingninganik inungnik.

Kujagênartaulutillo;

Pigimariktiguttôk

'Jêsuse akkiksarnut.

\section{Mel. 221.}

Wie lange muss Jesus doch. 421.

Ômativut parngnanærsimatillugit

Jêsusib pattakattarpait, Siutivullo simiksimatillugit

Kângertikattalaúkpavut, Soraingilarle saimanermut Kajungersatsainaramittigut,

Ômativut mangailijungnaitilugit,

Ikkiminut pissiarilauramigit.

\section{Mel. 97.}

Wir wissen alle wer wir sind. 422 .

1. Kaujimavogut tamapta Attunit ajudlarapta, Jêsusib naglikpâtigut Nâpkigosutuinarmut. Nâlegak nakoridlarpaptigit;

Nanêkita, Jêsuserangipat?

2. Mânna erkarluarlavut Igvit kivgarigaptigut, Orâlatigiguptigit
Pitsartutitsainartigut;

Okautigiguptigit inungnut

Jêsub Anernernut najortigut.

\section{Mel. 195.}

Treuster Fround der Seelen. 423.

1. Illannâk tarninik,

Ômat naglingniktor!

Illa tattamnadlarpotit,

Pittakatsangilak

Nunane tamaine

Okarungnartomik igvit

Ungagerkâramitit

Amuartaunane

Illingnut naglimut.

2. Illa opinarpor

Issumagijaukpat,

Igvit, idluartôjotit

Ivsornaitotillo

Pinasuaravit

Tarnit kenneromavlugit

Kemmâjut illingnik,

umigijitillo

Ajungivigangne.

\section{Mel. 68.}

Dank sei Jesu Huld. 425.

1. Ajulernivut Angijoksuit SakKertarput innôtseme, 
Jêsub nâpkigingmattigut Kennuênermut, Kujagilavut.

2. Kujagivlugo

Pingikuptale, Tâpsoma tunnergutingit, Naglingningalo pivlugit, Piunginivut SakKertipavut.

\section{Mel. 36.}

Ich bin in Wahrheit eins der schlechtsten. 426 .

1. Ahamarik ajortokullôvunga

Jêsusimale nachogingilanga,

Saimanermullo Kennilaurivânga

Okperkovlunga.

2. Jêsusible nellipsalaungippanga

Illa nellipsarajalaungilara, Nagligosungnermut KaiKolaungmanga, Kujagivara.

3. Mânna ômatimne kujagivara,

Pijomanerminut Kennilaungmanga,

IllagêktoKo timinut âmanga,

Kujalivunga.
3อ̈4. Mel. 79.

Mein blutiger Erbarmer. 427.

1. Aulik nâpkigijiga!

Suna pitjutigiviuk Aitilauramgale Illagêktokotingnut Tokkungnik serrijunut? Sunamik nagvârviovîk?

2. Ajulênadlartunga, Tarnimne ajortunga, Tikkinapkit igvit. Ômame tokkojunga, Aungnut inûlivarma, Pivlunga âniagavit.

\section{Mel. 22 a.}

Die Treue Jesu hört nie auf. 428.

1. Jêsusib Kennuêninga SokKositsængilak illa! Tamanna inôsiptingne Sakkijârpor illûnâne.

2. Illa kattakattarapta, Nâmagijanganut ôma Inôjuksautsainartogut; Kiglormut kiksartipavut.

3. Tamapta ingmikôrluta Tuksiarpaptigit mânna Naglingnermut erkittigut Ônartikit ômativut. 


\section{Mel. 228.}

Ich weiss, dass du barmherzig bist. *570.

1. Ullapirsartiga aulik Nâplkigosuktotôgavit Illitarivagille:

Ajortoêjarlungalo

Ullapirsarluaramga;

Kujalidlarlungale;

Illa tagva opigomut pâmakpunga

Kugvinulle

Kunnikpakka ittikkatit.

2. Illagêktut akkorngænne Mânna serridlarpungale, Aut kovigangne pivlunga Igvit nakoridlarapkit, Illagêktitavillotaur Illasearivânga ;

Illa tagva kuvianak

Nakungmêk, Gûb Kennermanga.

Illagênut pitimmanga.

35\%. Mel. 10.

Ach Jesu, Deine Treue. 429.

1. Jêsuse nâpkiningnît,

Kaut tamât nutângongmat, Kanortôk nâmaktomik Nakoritsainarlagit.

2. Kanoktôk! saimarsainît

Pijakkalo illingnit, Pivlugillo uvanga Kujalitsainarlanga.
358. Mel. 15.

Wie preis' ich doch den Liebesrat. 430 .

1. Jêsusiga nertorlagit! Tunnitsivigigamga Piluartomik illingnit Kennuarilauktamnik.

2. Naglimut kellaksulaukpat

Tarnigale illingnut

Kanoktôk soKkosingile, Paunga tikkerkârnanga.

3. Kanoktôrle nunamiut Illûnatik ingminik Tunnidlarlit Jêsusemut Perlugit agviartut!

4. Tagva nellonangitomik Kikkiaktortaulauktub Aggangminut puttolingnut Paunga pitinajarpait.

5. Kiægungnernut aulingmut Pissiarilauktatit Nagliklutit tuksiarput Pâmaktervigilutit.

\section{Mel. 101.}

Komm', beug' dich tief, du Volk des Lamm's. 431.

1. Nellopkotimut raititse, Illagêt Jêsusib inungit! Nâpkigijauvingmêtose, 
Tokkungmat ajortut pivlugit;

Tâpkonunga ikkingit angmarput,

Tigulerpait pingit okpernermut.

2. Illûnasele illagêt!

Nakorissiuk nagliktisse;

Kejungme tokkomut aglât

Naglingmagit pingortitane,

Tâpsominga nippangêrasele,

Naglingninga tamainut pingmalle.

3. Annerimattigut ôma, Nertortaule! pêjarmattigut

Kivgaujungnaitilutalo Ajortunut; pititaujogut Illauluta piulimajunut Kaujivavut ômatinga mânna.

\section{Mel. 79.}

Wir wissen niehts zu sagen. 432 .

1. Orausiksaeropogut Sângne kangusulimut, Erkailerpogulle Ajortoâlojogut, Pijungnaitinaptigut, Illingnullo kaikovluta.
2. Taimaimat ômativut Tamaita opigomut Kibliktaudlalerput: Ijivut Kugviorput, Jêsusib naglingnermut. Pissiarilaungmattigut.

\section{Mel. 208.}

Blutiger Versühner. 433 .

Aulik Jêsusiga, Ullapirsartiga, Auggit ikkitit Salutidlarpakka Kugviudlarnermut Annerigamga; Attê Kujalinermut Aniavit akikiksanganut. Ômatiga piuk.

362. Mel. 155.

Lamm, du hast die Welt gemacht. *578.

1. Nâlegak pingortipat

Silla, pingortiparmalo, Illagêllo

Pissiarilaukpatit,

Pivlugit

Tokkolauravit kejungme, Sângnelo innôKovlunga Ikkingnut inûlivarma.

2. Sillarsoarmiulle, Kattadlarningit pivlugit, 
Gûd illingnit,

Ajæktoromagungne,

Kiale

Agviarajarpâtille?

Piuligangnelo aungnut

Nakkoridlarpaptigit.

363. Mel. 45.

1. Nâpkiningnît angidlarpoK, Jêsus Piulijivut;

Naglingnit illuptingne Ikpigidlarpavut.

2. Serriluta kujalimut Nertudlarpaptigille, Nagliktok Nâlegavut Pikit ômattivut!
3. Kennuvogut, Jêsus, aungnut,

Ubvatsainartigulle,

Ajorungnaitiluta

Nertortaukovlutit.

4. Jêsuse, aut ivsornaitor

Kovigangne pivlunga,

Kujalijutigællugo

Kuviagivara.

5. Kissiane, mânna sulle Tammarnevaksoakka Kiksautigênarlugit Kugviorivakka.

6. Tokkogavit, âniavit Idluinêjarpânga: Tagva kissiat mânna Tungavigivara.

\section{JÊSUSIB INÛLERNINGA TOKKUNGALO PIVLUGIK ÔMAMUT KUJALINERMIK.}

\section{Mel. 114.}

Das Gotteslamm, das heil'ge. 435. Ivsornaitub Gûdipta saugârsungat

Pitlaræksaungitublo, aungminut

Gûdemut ullapirsarmattigut; Sillarsoarmiut nâlegæksarivât,

Illûnatigle kujagilitsuk Opigivlugo nakorilitsuk.
365. Mel. 58.

$\mathrm{Du}$, für die Sünder gebor'ner. 436 .

1. Kujanarle! inûleravit Ajortuggit piulivlugit, Ajortoliktitulle Timekarlutit Uviniktârlutit auktâravit Uvaptitut.

2. Piulijiksakalaungilak Assingnik, illale inungnik; 
Taimaimallo igvit

Tikkilaukpotit

Piuliklernermik kaitsilutit

Uvaptingnut.

3. Naglimut nellekangitomut Tunnergutauvotit pivluta, Ajornivut pivlugit Tokkolaukpotit Kejungme, saimanekserrovluta

Hallêluja.

4. Piulijaujose tamapse Hallêlujâdlarkattautitse Opigosukluse Imgeritsele :

Inôkovluta erniangovor Gûb Erninga.

5. Amen illa nertudlarlavut: Gûdipta illagingmattigut Ânianga kissime Pitjutiksauvok Avitauniatsænginaptalo Tâpsomangat.

6. Atâtab tallerpianêpor, Ikkingillo kaumatsainarput

Illagêktunelo

Okpernekartut

Kippilertut tikkiviksanganut

Missigivât.
366. Mel. 22.

Das Lamm, der Gott der Kreuzgemein'. *582.

1. Saugârsuk, Gûd illagênut Uvagut tættigijavut, Abrahablotauk Gûdinga Kinguvângitalo illa.

2. Tamât taipkoa sivorlît Naglingnartullo Profêtit Gûdigênarpâttauk tâmna, Toknomut aijos pivluta.

3. TaimaitsainaromârivoK Gûdigivât ajulertut Uviningmêtut illagêt Angergarsimajullotauk.

4. Tâmna pituarivavut, Assianullo ungajut Illagingilavut illa! Gûde tâmna pigaptigo.

\section{6\%. Mel. 230.}

Unsre Seel' soll Dich erheben. 437.

Inôtigidlaraptigit

Jêsuse nakorivaptigit, Takкavut tiglertillugit; Jêsus, illagigaptigit, Kujagitsainadlarpaptigit Naglingnivit pingmattigut, Kuviasulerta!

Nakoridlarlugo Sorairata; Hallêluja, Hallêluja Jêsuse Inûb Erninga! 


\section{Mel. 82.}

Bethlehem und Golgatha. *Anh. 24.

Betlehem Golgatalo

Pinnarivaptik illa,

Illiptingne tækkogama

Gûde Piulijigalo

Inûlermat tokkungmat

Pivlunga! opinarmêk.

\section{Mel. 121.}

So lang' die Hütte steht. 438.

1. Nunamêtilluta

Jêsub ânianga

Nakkoridlarpavut,

Tuksiarlugolo

Angergarkârtinnata

Kristusib aunga

Pijomavlugo

Ômatiptingne.

2. Jêsus, Nâlegavut

Ivleridlarpavut,

Ômamut tamânut.

Tokkunga aungalo

Okautigidlarlugik

Tagva inungnut

Pijomavogut

Piulingmagit.

\section{Mei. 151 i.}

Gott war's, der mich erworben. 440 .

Gûb pissiarivânga Ânialerlune
Gûb tokkotigivânga

Kovilugo aune.

Nagligijæksaunanga,

Nellopkotijunga

Gûdib nâpkigællunga,

Taimak naglikpânga.

\section{Mel. 147.}

Für uns verwund'tes Lamm. 441.

Saugârsuk ikkilik!

Pivlutale pigavit,

Tailugo nâmaktomik

Ajudlarpâ inûb,

Kejungme tokkogavit

Inôkovlugo inuk,

Ipaksilugole

Ajornarpok illa.

\section{3\%?. Mel. 119.}

$\mathrm{Du}$ bist's wort. 442.

Jêsuse :;:

Kejungme tokkogavit, Timiptingnut tarniptingnut

Nertutsainarlaptigit,

Omatipta sorairatik

Ungagênarlitit kissivit Tamainit :,:

\section{Mel. 205.}

Alles Heil wird uns zu Theil. 443.

1. Kejungme tokkogame Tamainit âkikpâtigut, 
Gûblo nâp-kiningninga

Piksarivavut tokkungmat. Aungallo kovimajub Sujungaitipâtigut, Illa! nâksaungitomut Piulijaumavogut.

2. Jêsuse tokkotaujok Kejungmele naglingnermut;

Illagêt orarilit

Sorairatik âniarnik.

Aungnut kovimajomut

Kiblikit ômativut,

Kidsijârnernut aungmik

Illuvut ônartikit.

3. Glôria, Hallêluja Jêsuse Piulijivut! Nertornak, tokkotitak, Inungortor Gûd, illavut, Tâmna illagêkluta Kujagivavut mânna, Angervigællugolo Nalainaromavlugo.

\section{Mel. 126.}

Das ist's, verwund'te Liebe. 444.

1. NaglingniktôjoK! tagva

Kibliktigivara,

Erkarapko, Jêsuse

Tokkotigigamga

Aut kissiat inônimne

Piloreutigivara

Nâksaungitomullo.
2. Illûnatik âniatit Illumnêtsainarlit!

Ungagijomavagit Tamât inôsimne, Ikkitit tautukôrapkit Kuviagidlarpakka Inôtigigapkit.

3. Kugviornermut mânna Kujagidlarpagit, OKausiksarivara

Uvamnut saimainît; Serngnigênaromagumga, Kivgartoromavagit Kuviagijarnut.

4. Idluartub Gûb aungat Omatimnit mânna Ajornikka pêrmagit, Missigimavara.

Taimaimat, inôtillunga

Puigorungnaipagit,

Pissiarigamga.

5. Okautigilerpagit

Ovanêtillunga

Opigosumut illa,

Nelloprotijunga.

Puigorungnaipungale, Aggut kovijaumangmat Piulidlarlunga.

\section{Mel. 15.}

Ach König, gross zu aller Zeit. *597.

1. Nâlegak ussornaravit Pinnarnarnerôvotit, 


\section{pivlugik ômamut kujalinermik.}

Aunâravillo pivlunga Nertudlatsainarlagit!

2. Nertorungnangikupkillo Tussugituinarupkit: Igvit tokkotigijiga Sulle nâpkigivarma.

3. Aggakpîllo saimarpânga Tarnib pagrearpattit; Timiblo nertudlarpâtit, Kusserviokpat aungnik.

\section{Mel. 15.}

Dank sei Dir, theures Gotteslamm. 448.

1. Illa kugviudlarnermut Kujagidlarpaptigit; Nâlegak Piulijivut! Pivluta tokkogavit.

2. Kennerialauraptigut Nâpkigosudlarnernut, Illagêtitarnullotauk Ovunga ânaptigut.

3. Aukpit, aukpit kovimajub Kajungertidlarpânga, Tunnilugo ômatiga Illingnut Jêsusiga.

4. Aunâdlartunut ikkingnut Najutsainalaungminga, Kikkiaktortauvingnelo Innekatsainarlanga!
5. Ániarnut tokkungnullo Tarnivut kivitikit, umativullo kaut tamât Aungne inôluarlit.

\section{Mel. 36.}

Du Gotteslamm voll Schmerr.

1. Gûb saugârsunga ânialaurtotit

Ikkekadlartotit auliksoarnik,

Tarnivut kellaksimavut illingnut

Kellugaptigut.

2. Mânna aupta kuttingita illûnatik

Kujagitsainaræksaridlarpâtit ;

Pissiarigaptigut, perkutingnut,

Illa tokkungnut.

3. Jêsusiga! Kujagivagit mânna,

Piulivlunga aungnut ubvaramga ;

Tâpsomungalo sapputilaungminga

Tammarkonnanga.

378, Mel. 75.

$\mathrm{Du}$ lieber Gott, ich wüsst. 449.

1. TokKokârtinnanga Illa nellovunga, 
Jêsusib assianik

Piuliklertomigle:;

Issokangitomullo

Tættigijomavara.

2. Tâpsomanêkuma

Terlikpunga illa,

Ajornerit uvamnik

Sâlakarniangilat,

Ikkilik erkarupko

Nukkingêrotilerput.

\section{Mel. 58.}

Dem Lamme gebühret doch alles gar. ${ }^{*} 601$.

1. Saugârsuk opinarpoк illa, Tunnergutaulaungmat pivluta,

Nâlegar tamainut

Kovisilauкрок

Aungminik, piulijaukovlugit

Ajortuggit.

2. Kanoktôrle ânianitit Erkaumatsainarlakka mânna!

Puigornangalo

Piuliklernit

Pingortitarnik, asserornermit

Aktornarmat.
380. Mel. 141.

Unsere Seele lebet. 450 .

Tarnivut ômavut, Umativut serrivut Sakkijalerangat

Kristus Piulijivut, Ingminik tunningmat. Ajornivulle

Pivlugit, tokкomut, Kovivâ aune.

\section{Mel. 185.}

Jesus hat uns bis in Tod geliebet. 454 .

Tokkomut Jêsub naglikpâtigut,

Aungmik kidsijârlune:

Tamattoma kiblingippattigut

Tâmna nagligilugo,

Ônarsingipattalo illuvut Ungagitsainarlugo uvagut, OKausiksêropunga Assianik nellogama.

\section{Mel. 79.}

Sein Tod macht Liebesschmerzen. *609.

1. Pivlunga tokkogavit Jêsuse! naglingnernut Kibliktaudlarama, Nutâmik inôserlo Illingnut tunnilugo Issumangnut attorminga。 
2. Pingijainer pêrlugo Aungat ubvarpâtigut, Umisuktiluta

Ajortunik tamainik, Attitipâtigullo Jêsub pinnarnarninganik.

3. Taimak Jêsub aunganit Ajungitut tamaita Pitsainadlarpavut; Pekarajangikupta Aunganik, tagva illa Nukkekarajangilagut.

\section{Mel. 218.}

O Du für mich ein Mensch geborner.

Pivlunga inungorsimajok Nâlegaviksôjor tâmna Annerijiga pinnarnartor Illannâtuarijara:

Illa pivlunga aunârtotit, Kejungmelo tokkolauravit, Illuvermut illingmattit;

Taimaidlutit kemmergolagit,

Illa tautukôtsáinarlagit!

Tarnima piksaringmattit.

\section{Mel. 121.}

Nun Lamm für uns verwund't. ${ }^{*} 610$.

Jêsuse ikkilik!

Tuksiarpaptigit,

Najulaungmittigut
Nungusuitomut, Ta mapta erkittigut Sarkijârille Ômatiptingnut. :,

\section{Mel. 97.}

Wir danken Jesu allerseits. 455.

1. Jesuse, âniadlartor

Ômamut Kujagivavut,

Ajorniptingnit tamainit

Illa piulingmattigut;

Gûde nakoritsainadlartaule!

Jesus pingipat, assiogapta.

2. Illa ômamine inûb,

Missigidlaleruniuk

Ajortomut sittigame;

Piulijipta aunganulle

Aumagune, nekkoksidlalerpok

Inûlingmago Jêsub aungata.

3. Aggut Jêsuse pingipat, Inôsek Kuvianaipok, Nimniuvigivavut nuna

Erksimut tikkiviksarnik; Mânna ikkingne nekkorsivogut

Sorairata tamaitsomârpogut.

4. Okautigilavut tagva Piulilauktipta aunga! 
Nagliklavut tokkuptingnut

Piulidlalaungmattigut;

Sorairata nungusuitomut Nakorilavut, naglingmattigut!

\section{Mel. 185.}

Um den sel'gen Umgang mit dem Einen. ${ }^{*} 619$.

1. Aiparitsainaromadlarlugo Keasôngolerpunga :

Kugvingita mannigudlarpânga

Ungamut kugvioruma. Kiksarnera pêjarpâ aungminut;

Ômatiga missigingikune Ânianginik, tagva Sannikterivor illa.

2. Tækkonârninganut ânanâmut

Kejungmille uvamnik

Ijîkka kugviortitauvakpuk,

Ânialik naglikpagit!

Jêsusima ânianingine Nekkorsijutiksakarpor illa

Ânanânik tarnimnut, Piloreutiksamnut.

\section{8\%. Mel. 151 a.}

Ich will jetzt mit Dir gehen. 458 .

1. Aiparijomavagit

Golgatamut mânna;

Tækkolago pivlunga

Mâne pijaulernît.

Tussunermut ômame

Malligomavagit,

Ijikka Kugviorpuk

Erdligidlarapkit.

2. Pitipât Jêsusiga

Kappianartomut;

Ajornipta sunaubva

Âtipât Gûdivut,

Nellojut agganginut,

Erkartuivingænnut:

Attorpait ânernartut

Attorajartavut.

3. Igvit kejungmêdlutit

Tuksiarupattit

Ipkoa ômisuktut;

Jêsus kinaulaukîk?

Erkarpattit illatit;

A ma erkaringa!

Inuartok saimarpat;

Taimaktauk saimanga.

\section{Mel. 185.}

Tausend Dank, Du unser treues Herze. 460.

1. KujanaK nagliktivut Jêsuse!

Akkisartoraptigut 
Âniarnut kappianartunut, Opigidlarpaptigit;

Mânna tautungigalloarpaptigit

Ungagênaromavaptigille,

Tagva aivigilutit

Tautugomârpaptigit.

2. Tarniga ajoksartok torầrpor

Ikkingnut Jêsusiga!

Tagvane mannigortiksarsingmat,

Nekkorsititaungmallo.

Tokkonît tautukôtsainarlago,

Timîllo aulik samnênarlaule!

Tagva angergarkârnanga Pilorijarêrpunga.

3. Ovane kattimajogut mânna

Angerкattigêkpogut Jêsub ânianga sorairata Nertoromagaptigo.
Nellonailivigilaungmittigut,

Kuviagidlarangne makkoa,

Imâk oKalauritôk:

Ullapkosertautitse!

\section{Mel. 126.}

Ich fall' zu Jesu Füssen. *620.

1. Jêsub ittigganginut

Pâmakterpungale,

Kugviudlarlungalo,

Kujalimut illa,

Ingminik tunnidlalaung-

\section{mat}

Ajornikka pivilugit,

Tokкomut Kejungme.

2. Sinnigomaleruma,

Pigârumalônêt,

Jêsusib ânianga

Erkaromavara.

Mattomanêtillungalo

Kujagitsainarpara,

Piulidlarmanga.

\section{JÊSUSIB IKKINGINIK OKPERNERMUT T AKKKONÂR-} NERMIK.

390. Mel. 23.

Jesu, weil in Deinen Wunden. *623.

1. Jêsuse! auggit ikkitit Merngoêrvigênarapkit,
Tâpkonanêtsomavunga

Illingnullo okperpunga.

2. Tækkonanga nagliklutit Najortigijomavagit; 


\section{Ómamullo erkilutit Ungagênaromavagit.}

3. Ikkitit kemergogapkit, Taimaktauk kôktut kugvitit,

Tagva nuname uvlukka Kuviasuklunga nâvakka.

4. Ikkitillo sorairnanga Nertutsainaromavakka, Pivlugit kuviasuklunga Illingnut angergarpunga.

\section{Mel. 10.}

Ach gönn' uns alle Tage. $*_{624}$

1. Pivluta tokrogavit, Tuksiadlarpaptigit: Aulingmut ômatingnut Ijertorniartigut.

2. Ijîngnillo kugvitit Akkusimajut aungnik, Aniallo, ikkitit Tarniptingnêtsainarlit.

\section{Mel. 167.}

Deine Wunden will ich kïssen. *625.

Ikkitit kunnidlarligit, Opigijomavakka, Ômatîllo sêksimajoK Salutitsomavara:
Tarniga torkortilauruk, Ikkime sennerarne, Terlitsainarungnarvimne. Ajortunit tamainit.

\section{Mel. 79.}

Ich kann's unmöglich lassen. *628.

1. Erkitsomadlarpagit

Allaromairlutillo

Piulilauramga,

Aulêglo ulluakik, Ikkitillo aunârtut Issumagênarpakkale.

2. Saugârsuk saimanernut Ômattimnêtsainarit, Kaumartilungalo. Inôsimne ikkitit, Âniajorlo timît Missigitsainarlakkatôk!

\section{Mel. 124 .}

Still' Du meine Sehnsuchtspein. *629.

1. Ungagidlarapkille, Jêsusiga,

Aungnut missuktênaruk ômættiga;

Ovanêtillungalo - najulaunga

Tækkôterrâranuk.

2. Jêsusib ômattiga najormago 
Inôtigidlarpara-kissialle; Missigingikupkolo-inônimne,

Ajoksadlarpunga.

3. Inûlijiksamigle assianik, Nellogama nuname, Jêsusiga!

Inulinga ikkingnut, ômattiga

Aungnut missuklugo.

\section{9อ. Mel. 166.}

1. Ômatimnut kannimnullo Kujagitsainarpagit, Jêsuse Krist ikkitillo Inûlijigigapkit; Illiktullo ômattiga Itsainarkovlugole Ikkingne, âniarnelo Nuname inôvlüga.

2. Tokkungnullo ikkinguullo Najutsainaleramga, Nuna taimak kemmakupko

Illingnullo piguma, Nungusuitoksoarmut Nakorijomârpagit, Puttomajut ittikkatit Pâmaǵvigilerlugit:

\section{Mel. 23.}

Nichts ist an mir, nichts als armes. $* 634$.

1. Illûnapkut ajorpunga, Ômatekartilaungminga Aungnut ônarsimajomik, Tokkungnullo aumajomik.

2. Issumiakka piniartakka Torâtsainarlit tokkungnut;

Ômatiga nippingale Ikkingnut tamât uvlumne.

3. Tautuvîllo nairtillutit, Ijimnut tautukôrtama, Illa âniatit tamaita Nekk jksijutigilakka!

\section{9\%. Mel. 75.}

Ein armes Sünder-Herz. $\quad 635$.

1. Ômat ajortolik Kappiasuktorlo, Sâtanib torkluanit Sapputijungnangimat, Jêsusib ikkinginne Piulijauneкsivoк.

2. Najuleraptigut Kuviasukpogut, Erkilerangaptigit, Naglingnît tukkisivarut; Ikkitit tautuklavut Tamât inôsiptingne. 


\section{Mel. 217.}

Was ist die lieblichste Figur. 463.

1. Tækkoranernerpâk suna Ânanaunerpâglo inungnit?

Tagva Jêsusib tautua, Illûnane âniadlartub. Tâmna ômamut illagêt Tækkojominartôningat Kibliktigidlaramitsuglo Aunga nekkoksijigivlugo, Serrilerput tarnimikut Gûd taimak tækkogamitsuk.

2. Ikkingit tautukkaptigik, umattivut kibliktaulerput, Tâpsoma ômisuktomit Amungmattigut, annerimut,

Uvaptingnut angernine Sakkertimagit naglimut. Ikkingita aunârninginut Sapputitsainadlarlittigut, TækkoKârnagole pâne Ânanaugijavut mâne.

\section{Mel. 68.}

Das ist moine Lust. 464.

1. Kissiat tagga

Kuviagivara,

Gûb sauganga ômatimne Sakkijârangat, kejungme Tokкojor sorlo, Aunârlunelo.
2. Ânianingit Ikpigivakka, Aungalo ittigganginit Tarnima mamagidlarpâ, Aggait puttoggit Salutipakka.

3. Angut ikkelik!

Ubvakit aungnut Illuvut uvlut tamaita Ômatiptingnêtsainarit Allaikonnata Illingnik illa.

4. Jêsus illingnut Tuksiarpogut, Uvlut tamaita ikkitit Uvaptingnut nutaulerlit, Puigorkonnata Piuligaptigut.

5. Illa kejungmut Sâptingne sorlo Kikkiæktorsimajotit, Tautukôrpaptigit tagva; Saimarsautitit Tukkisilavut.

\section{Mel. 184 a.}

Ein Blick im Geist auf Jesu Leiden. 465.

1. Ômattipkut mannigorpunga

Gîd âniat tautukupko; Illuma kuviasûtinga 
Imaipok : korTorvigigungne

Aungnut ômatiga Jêsuse,

Kiægukpit kiblikpanga,

Okpernermut erkilerup-

kit,

Tautuvillo saimarpanga.

2. Jêsus pivlunga tokkolaukpok,

Pivlunga aunga kovivok; Tagva ômatib Kêdlarninga Nâmaksititaumarikpok.

Kôk issungitok ânanaujor!

Ômatimnut kôtsainarit,

Puailaviglo saimanelik

Kusservigitsainalaunga!

3. Senneranganêtsainarnermik

Ômamut Kêmitikpunga:

Nâlekab âniaviane,

Milluartotun-ilanga.

Aggait illipsingne atterma

Aungmut aglaksimavingit,

Nagliktamnit tokKovik-

saminut

Kemângitsainartissinga.

4. Jêsuse! pigiluarminga Akkilliutigilunga.

Erkaumajæksakarlangatôk

Ômatimne naglingnernik,

Pititigijarnik tokkomut,
Piulijaudlarkovlunga:

Kanoktôk ungagênarlagit!

Tussaramga kaujivunga.

5. Tautugungnaipannik ijîkka

Ikkingnut torkortiminga Okarungnaileruma mâne Kaijomavunga Jêsuse! Timiga illuverme tagva Merngoêrluaromârpox; Tarnigale ikkingnut illa Angergaromârpok tagva.

\section{Mel. 9.}

Bis dereinst mein Stündlein schlägt. 466.

1. Nelliukârtinnago Angergarviksara, Illijaukartinnago Time illuvermut;

2. Jêsub ânianingit Opigidlalugit, Ikkingit nertorlugit NokKaromaerpunga.

3. Okperlunga tarnekut Allagaigilugo, Nakorijomavara, Nippimnut nellâgut.

4. Piviojuksaudlarmat,

Illûnainut illa

Kilangme nunamelo Nortortaukovlugo. 
40?. Mel. 228.

Wie lieblich klingt mir das Getön'. 467.

1. Tarnimnut tussarnernerpâk

Jêsub ikkingit tamaita,

Nertortaungmatta tamât;

Ômatima serrigivait

Missigijarangamigit;

Âniajor Gûdiga!

Tagva illa! ômatipkut

Kujalimut nertorpagit

Ovane pânelo sângne.

2. Saugârsuk ikkeliksoar

Nâlegara Gûdigalo!

Ikkingnik naglikpunga.

Akludlaralloarpunga

Oкarungnatsiarnangalo,

Sulle serridlarpunga,

Mâne ikking-ne illale

Nagvârama sernârnermik

Tokkomit ajortunillo.

3. Mannigornadlartok tagva, Jêsuse nellunginama Tokkungnut piuligamga! Missigigapko tamanna, Tagva nunab pingit illa Nachogitsainarpakka. Illa tagva, pilloringnak, Kuvianak, Jêsusiga, Pigigamga pairællunga.
4. Jêsuse! nâksaungitomut

Kujagivagit nellâgut, Âniatigigamga!

Auggit ikkitit tamaita Sorairnanga kunnikpakka, Tâpkoa piksarivakka. Ikkingnut mâne pingitut Okalârput : каккâluit Mattortigut erksigapta.

\section{B. Mel. 151 a.}

Das mächtigste Gereitze. ${ }^{*} 647$.

1. Jêsus ânernangêrmat

Kejungme pivlunga

Tamanna ômatiga

Kibliktautigivâ;

Sâpunga ikkinginut

Erkomaleruma

Sivorlermik tâpkoa

Erkaumadlarpakka.

2. Pinnarnartotôjotit

Jêsus! erkilagit

Ketserartautillutit,

Pinnaridlarpagit,

Avatitit Kejungmut

Issaktitaumajut,

Kunnikpakka kugvinat,

Salutidlarlutit.

\section{Mel. 75 .}

O Opferlämmelein. 469.

1. Gûdib saugârsunga! Piksarsiorpogut 
Sorairata ikkingnit Inôgutiksaptingnik; Uvlullo nungulugit Sapputitsainartigut.

2. Aungnut Jêsusiga! Illijaulaulanga:

Aggait issammijose Naglimut tigusinga, Pairisinga, sunamut AкKonartaukonnanga.

\section{Mel. 126.}

o Lamm, Du Herz voll Liebe. *649.

1. Saugârsuk naglingnernik Tættæmat ômattit;

Aumavunga illupkut Taimak tautukkapkit, Illa! pitlaræksaujunga, Tokkogavit, Gûdemut Nâpkigijauvunga.

2. Kujalita! Gûb aungat Piulingmattigut, Illa taimailaungipat Pitlaræksaujogut, TokKolaungipat pivluta, Amuluta tokkomit, Assionajarpogut.

3. Ômatiptingnut mânna Âniatit aglakit, Jêsuse, illûnata Gûdigigaptigit
Tarnekut pairigaptigut; Ivsornaitut ikkitit Inôtigivavut.

\section{Mề. 58.}

Wir freuen uns über. $* 650$. 1. Jêsus Kutsaservigivavut Okpertunut kaikungmattigut;

Ôma ikkingine Ômatânelo Innekarluta pilorikapta Hallêluja.

2. Aungnut serparsalaungmittigut

Âniavingnut ârlorpogut, Aulingnut ikkingnut Ômatingnullo Erkitigut piloriktiluta Illûnâne.

3. Aggangnut puttosimajunut

Illûnata serparsartigut, Ittikkat puttoggit Kejungmut pijut, Jêsub avatingit ikkilertat Kunnikpapse.

4. Nâlegak nakorijaulaurit Pivlunga ânialauravit; Nipperarnangale Opigosumut 
Erkitsomavagit sorairnanga

Tautukârnæk.

\section{0\%. Mel. 79.}

Mein Wohlergehn im Herzen. 470.

1. Kuviasungnerale Omatimne pivara,

Jêsus âniarnit;

Mannigorneksigama

Ikkingne, tagva mânna

Nagligivakka tamainit.

2. Inuille Gûdivut

Illitarigunitsuk,

Pivlugit aunârtoK,

Ômame naglingningat

Onarsitinajarpait

Ômunga perkutaulutik.

3. Jêsus kikkiæktorpât

Kappiaktoatipât

Kittiksaungitunik;

Kugviormut tamanna

Tailugo sapperpara,

Ikkingit tækkonârsigik!

4. Jêsub ânianingit

Kikkiaktorningillo

Nagligomavakka ;

Aungểrotileruma,

Angergarniarlunga

Taututsainaromârpakka.

5. Innangalermiguma Ama makkikumalo
Sulligumalônêt,

Taimaktaur nerriguma,

Ingergalerumalo,

Ikkingit erKarlakkatôk!

\section{Mel. 185.}

1. Tuksiarpagit Piulijiga

Najunga Anernernut,

Kaumaksarlugolo ômatiga

Illitarilerlutit;

Tokkungnut aungnullo ikkingnullo

Kiægungnernullo keamullo

Piulidlalauramga

Puigorungnaitinga.

2. Illingnut adsiotinianga

Idluarsalaungminga;

Illingnut ungatsainarkovlunga

Tоккоmut naglikamga.

Auktun-itomik kidsijâravit,

Tarningne kappiaktoavlutit

Ajornikka pivlugit, Kujagitsainarpagit.

\section{Mel. 68.}

1. Aukpit kovijub

Timingnit kôktub, Ômativut auktiligit. 
Âniarnerpit auliksûb

Kiblilittigut

Ômatiptigut.

2. Tamaita uvlut

Auggit ikkitit,

Ômatiptingnêtsainarlit

Inûlijigigaptigik,

Attorkolugit

Kaut nungulugit.

\section{Mel. 82.}

Unser Lamm ist gar zu schön. 471 .

1. Pinnarnadlartotôjor

Illa Piulijivut,

Tautıkaptigo sajuktok,

Kappiasumut кеajok,

Aunârtorlo tokкomut

Pivluta naglingnermut.

2. Illûnatik kiksartut

Ajornitik pivlugit,

Jêsusible ikkinginut

Apkutânik nagvâlertut,

Nekkoksivut illume,

Tautuklugo kejungme.

3. Niakôsertaujorlo

Kakkilautiâlungnik, Mittautigijaudlartorlo, Âniatitaudlartorlo Ikkilertaujoksoar; Naglimut taimak pijor.

4. Illa taimaitsainarpok. Ajortut serridlarput
Piuliklertekaramik,

Tokrolauktomik pivlugit;

Pinnaridlatsainarpât

Kikkiæktorsimajoк.

\section{Mel. 217.}

Ihr Wunden Jesu seid uns all'n. *654.

Jêsuse ikkingnille illa

KôlitsainarpoK tarniptingnut Serrinermik angijomik; Sennerangnille aggangnillo

Kêlerluta Kallugupta Aungmik immermiglo, tagva Tarnivut nekkoksijaudlarput,
Piloriktitaudlarlutiglo; Kanoxtôlerpullo tagva Kaikojimut illûnainik.

\section{Mël. 126.}

Ihr heiligen fünf Wunden. 472 .

1. Jêsusima ikkingit

Ivleridlarpapse,

Illipsigut nuname

Kilangmiồjârama;

Mânna tamânêtsominar

Nâlegak nagligapko

Tokkotigijiga.

2. Serrijutigivara, Siarklernangalo; Sangujomaleruma, 
Âxisâlerpânga,

Omatiga pallangaerpâ

Itterviokonnago

Sujugutiksamut.

3. Âniangita kissimik

Ômisuktipânga,

Ajortunik, illale

Serlekinajarpunga,

Taimaitut ungagigupkit,

Nivingatitsilauktut

Ôminga kejungmut.

4. 'Tækkosarneк ikkingnik

Imanuêliga!

Kellalauкpor uvamnik

Illingnut illûnapkut;

Okperlune ungagijit,

Saimanermut nuname,

Pairitsainarivat.

\section{Mel. 90.}

1. Jêsuse âniarnulle

ToKkungnullo aungnullo

Najunga uvlut tamaita;

Pairitsainalaungminga,

Ikkitit soraijunagit

Tarnima innigiligit!
2. Aungnut pissiarigamga Tokrotigællungalo.

Pigitsainaromavarma Timipkut tarnipkullo. Ikkitit soraijunagit Tarnima innigiligit.

3.Kujagivagit Jêsuse! Taimak nagligigamga Illingnut inûlertinga Mattomanêtillunga. Ikkitit soraijunagit Tarnima innigiligit.

\section{Mel. 230.}

Illagêktose okokse! Kiksarnartokalerangapse Sumut mannigortauvisê? Ajoksarangapselônêt, SoKaraselo tarnipsingne, Namulle sânajarkisê? Jêsub ikkinginut Apkutekarpogut, Akludlartut, pattangajut, ajoksartut Ikkinginit piksarsivut. 
Jêsusemut naglingnermik tâpsomungalo etc. 181

\section{JESUSEMUT NAGLINGNERMIK TÂPSOMUNGALO KIPPINIARNERMIK.}

\section{Mel. 232.}

Herzlich lieb hab' ich Dich, o Lamm. 473.

Jêsuse nagligivagit

Kikkiæktortaulauravit,

Sajukavit pivlunga;

Tautukôrpagille, imâk,

Nuvuliktartudlartomut,

Niakut killertaungmat.

umunga kuviasumut

Ijîkka kugviolerpuk,

Omatigalo ônarpor,

Auksersimajotun-ipor.

Jêsusiga

Aungnut kôktomut ikkingnit

Ômatiga inûliuk.

\section{Mel. 224.}

O liebt ich Dich recht brünstig. 474.

Kanoktôk nagliklagit

Nagligerkâramga!

Naglingnikidlarapkit

Kiksarkattarpunga!:

Agulle Jêsuse

Kovidlalaurangne,

Ungagijæksatôvor

Tamainit illale.

\section{1\%. Mel. 167.}

Könnt' ich recht von Lieben sagen. 475 .

1. Kanortôk! nagligilago Nellâgut Jêsusiga, Ômamut ungagilago, Kammaginagit assingit, Illûnaita allarlugit Idluaringitangit;

Kilangmiôjâlerpunga Gûde aiparivlugo

2. Kanoktôk! tamaita uvlut Illumertaulaungmita Jêsusib naglingninganik, Ômamut nertorlugo; Timiptalo tarniptalo Kuviagidlarlitsuk! Piulijipta aungminik Missigitingmattigut.

\section{Mel. 97.}

Nun will ich mich scheiden. ${ }^{*} 664$.

1. Tamaita ipperarpakka

Nunab pingit nungusấrtut, Inôvunga nagliktamnut Pivlunga tokkolauktomut; Tâpsotunak nagliktokangimat

Nunamelo kilagmelo aglât. 
2. Jêsus erdligidlartara, Nagliktor Piulijiga!

Tamainit nunamêtunit Nagliklutit pinnarivagit, Tarnipkullo erkitsomavagit,

Kissivit merngoêrvigigapkit.

\section{Mel. 20.}

Jesu, Deiner zu gedenken. 477.

1. Jêsusiga erkarupkit

Kuviasuktiparma igvit, Najudlarumgale, tagva Kilangmêpunga sorlo.

2. Unêt okatsiarnanga Nippangerungnangilanga, Illingnilk oxâlalunga, Naglingniktotôgavit.

3. Magdalênatut-ilunga Nellipsalerpagit mânna, Najorlagit sorairnanga Kujagijomagapkit.

4. Nâlègara! najulaunga, Kaumasît kaumaikovlugo Tarnimne, târnêjarlunga. Nekkoksitilungalo.

5. SungiutineK illingnik Kaitsidlarpok kippinermik,

Ijik kugviortitauvuk, Ômat saimalaungârpor.
6. Immeruktut immikangne Kâktullo nerritinnangne Pêtut nachoginginangne Nâpkiginga Kaigama.

7. Atâtangnut ailaukpotit Ânanaunerne-ilutit, Anernipkulle tagvunga Malligomadlarpagit.

8. Tagvale inôtillunga Nertoromavagit mânna, Aivigigupkille tagva, Erkidlaromavagit.

\section{Mel. 83.}

Meinen Jesum lass' ich nicht. 479.

1. Allaromangilara Jêsusiga inônimne: Tokxotigilaungmanga, Inôjuksaudlarivunga Illale tâpsomunga, Kivgartorlugo mâne.

2. Sunamut assianut Killanângilak tarniga; Jêsusele kissiat Ullapirsartiga Gûmut Assiotailitiga Tussugituinarpâ.

3. Jêsusigale illa Kemmagomatsangilara, Nanele pittakarkâ Serrinartomik ôtunak? 
Pilorikpunga illa

Jêsusib naglingmanga.

4. Mânna nagliktigêmut

Pigivara, pigivânga;

Inôjutigivara

Aungmut pissiaringmanga

Illa tættigivara,

Sapkoniangimanga.

\section{Mel. 124.}

Seht mit frohem Angesicht. $*_{670}$.

1. Alliagidlarpara

Jêsusiga,

Nekkorsitigilugo

Pigivara,

Ômalo pigivânga,

Kissiallo

Issumagivara.

2. Tâpsoma erkilunga

Kunningmanga,

Perkutinit tamainit

Naglikpara ;

Kuviagidlarlugo,

Tâpsomunga

Inôjomavunga.

\section{Mel. 106.}

Ach, wem er seinon Kuss gegeben. 480 .

1. Jêsusemut erkitaulertub Ullapirsautekarlune, Missigijungnaileruniuk
Inôser tagva ajorpâ,

Nagvâtsainarpâ Jesuse

Kennervingmine tamaine.

2. KanoKtôk inuit attunit Ômamiutarilitsuk, Kanok ogguarnarmat, sulle

Uvlome torângimatsuk; Jêsub pissiaringmagit Aungminut, kôngmat pivlugit.

\section{Mel. 132a.}

O Jesu Christ, ich preise Dich. 675 .

1. Jêsuse nakorivagit Ômamut serrijomut, Aklujunga saimanermut Naglimullo toKkungnut

Piulitsialauramga Ajortunit, tunnilunga Idluarnernik aglât.

2. Mânna tagva naglingnivit

Kajungitênarlinga, Kippivigitsainarlutit, Ajornerlo ômamut Ulloreagênarlugo. Ajungivigilauramga Perkutigidlalaunga. 


\section{Mel. 15.}

Dein Kreuz, die Schmach, die Angst, der Schmerz. 483.

1. Tokkovît, âniavillo Aukpîllo ikkivîllo

Ômatiga auktingmatsuk, Illingnut nippipunga.

2. Tamakkoalo illumne Naglingnek ômartipât; Kaujigama tokkogavit Ajornikka pivlugit.

\section{Mel. 83.}

Gottes Lamm, so kann Dein Blut. *680.

Aukpit Gûb saugârsunga! Ômativut niglidlartut Onarsitipait tagva, Time tarnelo ungavuk Angijomik illingnut, Ullapirsaraptigut.

\section{Mel. 230.}

Ach lasset uns ihn lieben. 484.

Jêsuse nægligilavut

Ôma næglerkâlaungmattigut.

SoKKosilautsangimallo;

Kiksartikattalauktapta

Unangmivâtigut tamapta

Kellarudsâromavluta.

Ikkiminut illa
Inûlidlarluta

Tussudlarpoк.

Tagva mânna

Kujalimut

Nagligilavut ômamut.

\section{2\%. Mel. 200.}

Wie freundlich, selig, süss und! schön. 485.

1. Jêsusiga naglingnernut Idluarsitiparma, Ômunga kibliktauguma. Tâksiutsængilanga; Taimaimalle ômatiga Aumale næglingnernut, Sivorlermik Nagligilauramga, Ungagitsainarlagit.

2. Sapputilauruk tarniga, Illumertaukonnago Naglingnivit assianik, Tâmna annerilago! Annitikit pêrlugillo Avititsijomajut Uvaptingnik, Nagliktimingalo Illingnik issuanut.

3. Jêsusiga tokkovotit Naglingnermut uvamnik: Pijauvotit inuartotut, Kikkiaktortauvlutit, Ikkilertauvlutit illa. Attêle ômatimne 
Ikkit auggit

Uvlut tamaitale

Missigitsainarlakka.

4. Aullo idluartoksoak

Ivsornaitorlo tâmna

Kovimajorlo pivlunga;

Uvanga ingergarma

Ujarkatut-idlarama,

Ômattigale piuk

Auktilugo

Inûlivlugolo,

Aungnullo ikkingnullo.

5. AngmarkojominadlarpoK Ômattiga, ikkingnit Kuttit auggit korlolertut, Tamât piksarilugit;

Ajornima pitlarmattit,

Ijikka Kugviorpuk

Naglingnermut

Kippiniartotut,

Illaminut pivaktotut.

6. Nutakratut keajotut

Mallitsainarmilagit,

Ônarsilunga illupkut,

Tarnipkut erkilutit,

Kanoktôrle naglingnermut

Omatimnut Gûd igvit

Ungagilagit,

Attavigilutit

Inôvigilagittôk.

7. Naglikparma issuanit Illingnut amulunga,
Kiksartikattartagille

Ajungivigivarma;

Tagva tamât naglingnivit.

- Tessiutsainarlinga,

Illaginga,

Naglingnernut illa

NekkoKsitsainarminga.

\section{Mel. 11.}

Jesu komm doch selbst zu mir. 487 .

1. Jêsus! Kailaurit mânna, Ilkkingnullo najunga, Illa erdligigapkit, Ômatimnêtsainarit.

2. Pikkalo idluitut

Tunnivakka illingnut,

Issumagijungnaikit,

Ubvalaungminga aungnut.

3. Assingnut ômatiga Angmarniangilara, Illingnut Jêsusiga Angmarkojomavara.

4. Kissivit Gûb Erninga Itterkojomavagit, Kissivit pigivagit, Nâlekamnik taivagit.

5. Pivlunga Jêsusiga Kikkiaktortaugavit, Ômattiga ikkingnut Mannigudlalaungmiuk! 
6. Aniarnut tamainut Kellugangne tarniga; Aggut kôktor nunamut, Kusserletố uvamnut.

\section{Mel. $151 \mathrm{i}$.}

Schatz über alle Schätze. 489.

1. Pivianartok Jêsus! Tamainit illale! Serrijutigijagit: Mâne inniksamik Ariksoilaukpunga Ômatekullumne Illingnut, âniarnut Inûlilauramga.

2. Kuviagênarpagit, Kakкôjak kilangmit, Nekkorsitigivagit Kanoêdlarnimnit. KiksarnartoKarangat, Illumertiparma Mannigoinermiglo Mammadlartomiglo.

3. Kênat illannârnartok Tækkolago Jêsus Umatiga illumne Mannigorkovlugo; Najorniangikumga KiksarpoK illuga, Sângnênasuaruma Kuviasukpunga.

\section{Mel. 39 .}

Ich rühme mich einzig der blutigen Wunden. 493.

1. Jêsuse, pivlutale ikkilik angut,

Nertornautiksatuarijomavavut,

Torkorvigilugit ikkingit tamaita,

Sorairata Gûde nâlegomavavut.

2. Okorse! Jêsus pitsiarlune Kaivoк,

Naglingnermut erkitsomadlarpâtigut Næglingnarmêk! illa innôtsiarnarmêk!

Kina Jêsusemut ungajomangilâk?

\section{Mel. 39.}

Ich liebe Dich herzlich ${ }^{*} 696$.

1. Kissivit, Jêsusiga! nagligivagit

Ômatimne Kuviasugvigivagit,

Kenneramga, ômamut Kujagivagit,

Erkitsomavagit allaromaerlutit.

2. Tokkungnut aungnullo pissiarigamga, 
Tarniga illingnut perkojomavara,

Kujagidlarpagillo inôtillunga,

Nungusuitomut taimailiorpunga.

3. Illa Nâlegavut kikkiæktortaungmat,

Aungminullo piulidlalaungmattigut,

Ikkiminut illa inûlingmattigut,

Kujagivlugolo nakoridlarpavut.

\section{2, Mel. 142.}

O Ursprung des Lebens. 496.

1. Inôtsib issua, Kaumaniojor!

Illingne kennertor nagvâtsiarpok:

Puailaviojok, immek issungitor,

Nekkoksitiksaptingnik kôtsainartok,

Tarniptingnullo kêlertunut pijor.

2. Okarpotit: immeromajok uvamnit,

Soraijuitomik, kailerle! mâne

Tunnijæksakarpoк, nekkoksinartunik;
Okpernermut kaile puailavingmut,

Pilloreutiksanga tamânêpor.

3. Ahâk Pairijiga, saugârsûjotut

Kêlertotulle kaivunga illingnut;

Kaujimadlarparma ajulertôgama,

Taimaimalle, nagliktiga imminga,

Akkekangitoniiglo saimarlunga.

4. Ômativut aungnik immertipattit

Illunaita pitit inôtipattit;

Inôgutiksamik soraijuitomik,

Okpertulle tunnitsivigigangne,

Kuviasuklutik : kiksarungnaiput.

5. Sorlo okaravit, immertilaunga

Ullapirsarlugolo anernera,

Immiksar timingnit, aulingnit ikkingnit, Korlormat ajulertunut mânnakut, Ômattiga kêlerpor taimaitomut. 
6. Anianartomik pitikuptigut,

Tunnitsivigittigut nâlengnermik :

Ermgusiarsungmit immerrâlauktarnik

Illûnatik mâne immerkattivit

Kuviasukattigijomârmattit.

7. Taimaimat, Jêsuse nekkorsitinga!

Okpertut âniajungnaivingænne,

Kuviasugvingme allianartome,

Serrinarvingme ânanaunivingme

Akkilêvingmelo nertornarnermik.

\section{Mel. 124.}

0 was für ein Gnadengrund. 497.

1. Tungav ekarpok illa

Naglingnerme!

Soкrosijuiluta

Naglikupta:

Naglingneкangituble

Inôsinga

Sunaungilak illa.

2. Naglingniub pêjarpâ

Okumainek,

Sangênivut ômunga
Nukkekarmat,

Sullijæksakarnivut

Tamaitalo

Okrititaulerput.

3. Perkutaumarikupta

Jêsusemut,

Issumakarlutalo

Tâpsotunar

Kajungertitauvogut

Naglingnermut

Inôrigilugo.

\section{Mel. 146.}

Ich hab' ihn wahrlich lieb. ${ }^{*} 694$.

Illa naglikpara

Attavigilugolo

Kuviagijara,

Kippivigijaralo ;

Nellâgôrvigivara;

Tamânelo sulle

Ômamut pivlunga

SerrijuksaulerpoK.

\section{Mel. 58.}

Das unser Heiland liebt, ist bekannt: 498 .

1. Piulijipta naglingmattigut Ânianganit Kaujivavut; Illagêt naglikpait

Ajulertullo

Sorrutsillotauk nagligidlarpait

Ũvangálo. 
2. Taimak Jêsuse nagliktara, Johannesetut nagliginga! Magdalênatulle Salutipagit, Erkilerlugillo ittikkatit Naglingnermut.

3. Anânatut najuênanga Najungikumga Keavunga; Missigingikupkit Illa illumne, Ônarsingikuma ômatimne Ajorpunga.

\section{Mel. 79.}

1. Mâne inôtillunga Kaitæksakangilanga, Attausênarmigle
Pijomadlarpungale 'TokKungnik âniarnik Missigênaromavlunga.

2. Auktojox Jêsusiga! Ajoktokullôvunga, Illitarivarma; Tamannale pivlugo Sapputitsainarminga Nuname inôtillunga.

\section{3\%. Mel. 132 a.}

Nâlegavut nagliklavut Mattomanêtilluta, Tokkominut aungminüllo Piulilaungmattigut; ToKkominullo tâpsoma Tokkuksavut toKkonivâ Nâpkiniktoksôgame.

\section{KA'TTANGUTINUT NAGLINGNERMIK ATTAUTSE- MIGLO ISSUMAKATTIGÊNGNERMIK.}

\section{Mel. 11.}

Jesu, Haupt der Kreuzgemein'. 503.

1. Jêsus inukotivit

Niakorênarmattit

Tamaita okausernut

Tessiokit illingnut,

2. Inungnik pairksigavit Tamaita kattersokit
Tammartut, saimanernut Attausênangortikit.

3. Attausingortittigut Avitailitittigut Illa Kattangutitut Adsigêktimittigut.

4. Illagêktut tamaita Naglikattigêktikit 
Âxiksorluarlugit

Illingnullo inôlit.

\section{Mel. 161.}

O ihr theu'r erlösten Sünder. 507.

1. Piulijaumajosele

Jêsub Kristub aunganut

Kittorngaujutitut Gûmut

Ullapirsartaujose;

Okalaukta,

Nippekarta,

Pijariakarniptitut

Jêsub pitsartuninganut.

2. Saimanermik nagvârpogut

Saimanermik illale!

Erksinerle pêrsimangmat Kaumaneк sakкijârрок;

Ajornivut

Sorairtuksauvut

Okautsib uviniojub

Ajornerit pêjarmagit.

3. Saimanerub sakkijârtub

Kajungersarpâtigut,

Nâlegar kivgartorlugo,

Nagliktigêklutalo.

Saimanermik

Kaujingitut,

Tukkisitsialerlitsuk,

Ajungivionivulle.

4. Kittorngârsuit NâlegaK

Jêsuse tussarsiuk,
Tarninigle sâlakartoK

Nâletsialersiuk!

Okausingit

Kingorlerpât

Kigligiudsilaukpulle

Naglingnermik. Malliksiuk!

5. Pâmagitse Jêsusemut,

Kattangutigêktose,

Tækkorngartaulauktosele,

Mânna kannidlartose!

Kaujititse

Jesusible

Perkutsainalauktanginik, Nagliktigêngniksaptingnik.

\section{Mel. 167.}

Herz und Herz vereint zusammen. 505 .

1. Ômaterattigêkluta, Gûdib ômatingane Merngoêrneksiudlarta, Nagligidlarlugolo.

Niakôvok, uvagullo Avatigivâtigut

Nâlegauvok uvagullo Kattangutigêkpogut.

2. Kittorngaujose kaititse! Angervigidlarlugo Sâlakartivut ômamut, Sokkosijuinermik. Naglingnise ajoksarpat 
Nellâgôrnermik sulle, Tuksiarkattigêgitse Âriktaukârtinnase.

3. Kajungertitigêgitse, Saugârsuvut illavut, Pivluta tokkotaulauktor Ômamut malliklugo. Tuppârtigêktuksauvogut. Pitsartunivut tamât, Attutsainarlugo illa, Kuviagijanganut.

4. 1llannâK pitsiadlartoK! Attausingortikittôr Pitit ungagijitillo, Nagliktigêrovlugit, Sorlo pijomadlaravit. Illa miksekârnerme Illagêktimarilaukit Kaumaksarsimajatit.

5. Naglingnek! perkojigavit Naglingnek attorlugo, Tagva tokкungajutitut Inôsekartikittôk, Naglingniub ikkomanga Ikkitsuk illuptingne, Tækkuksaumarerkovlugo Ungagidlaraptigit.

6. Attausingortitiguttốr! Inôsiptingne mâne, Sorlo igvit Atâtallo Attausiudlaraptik. Ikkoalanernit illa
Kaumasivut kaumaile, Tagva tækkuksauniarpok Perkutigigaptigut.

\section{Mel. 221.}

Sobald wir von oben auf's neue geboren. 504 .

1. Anernermut erniangomalerapta

Kattangutigêlerpogut;

Kilangmullo ingergarkattigêkpogut,

Issumatârtitaugapta,

Tamanna pivlugolo illavut. Nachogitsænnagit, naglikpavut,

Gûdib kittorngane averKongimagit;

Perkogivait nagliktigêlerrovlugit.

2. Tamanna pivlugo nagliktigêmita

Kiksartinnagit illavut, Nagligitsainarlugille illûnaita,

Nâlekab perkungmattigut; Atâtab kuviagivâtigut, Ernek naglikaptigo ômamut;

Atâtavut taimak nakoridlarpavut

Naglingnerub innigingmagit illuvut. 


\section{Mel. 14.}

Der $\mathrm{Du}$ noch in der letzten Nacht. 506.

1. Unnuarme kingorlerpâme Tokrolerkârtinnæk, NaglingneK perkolaurangne

Tamainut illangnut.

2. Taimak Jêsuse, illagêt Erkaititsainakit, NagliktigêlerKovlugit PerKogangne igvit.

\section{Mel. 15.}

Da wo man wahre Liebo spürt. 509.

1. NagleKattigêtsiartut Jêsub pitsartutipait Kittornganelo nellâgut Tessiutsainadlarpait.

2. Nanelônêt-ikuptale Sakkijârtuksauvogut Ingergarkattigêktitut Aprome Jêsusemut.

3. Illavut tammarkonnagit Kaiblaleraiguptigik, Omativut pairilavut Nellâgôrrovlutale.

4. Nâlegar ikkajortigut KajungilârKovluta, Nellâgut kattangutitut Nagliktigêkovluta.

\section{Mel. 141.}

Schliesst euch mit Vergnügen. 510.

1. Nagliktigếgitse,

Perkutaujose

Ikkiliksoarmut,

Kunnunaselo

Attausiotitse:

Jêsusib aungat

Ômativut illa

Ôrartiligit:

2. Saugârsuk ikkilik,

Illûnaptingnut

Nertortauxovlugo,

Ikkomanivut

KaumaKovlugolo,

Ômatiptingne

Jêsusib ikkingit

Kaumaimangmatta.

\section{5๊. Mel. 166.}

Du Geist von oben flamm' uns an. 517 .

1. Anerner pângat pijotit Nagligosuktitigut, Tamât tammarkattarnivut

Kiksautigilerpavut; Ajortôgalloarluta Jềsuse, pigaptigut, Taimaimallo attît mânna Nertoromadlarpavut.

2. Naglingnekartimittigut Ullapirsarnartomik, 
Ômativullo illingnut Attausênangortikit. Illagêkluta ômattît Aivigigangaptigo, Idluinivut pêjakit Aungnut ubvarlutalo.

3. Jêsuse, illagêkluta Mânna tikkipaptigit, Nagliktotôgavit, illa Tuksiadlarpaptigit, Naglingnermik ômattivut Ikkomalerlit sorlo Soraijuêrtilugillo, Paunga perkârtinnata.

\section{Mel. 121.}

Gemeine, liebe dich. 518.

1. Illagêktut attê!

Nagliktigêgitse!

Ômamut nellâgut;

Kiglinganit Gûdib

Annerimalaungmasse,

Nagligilugit

Ningarijise

Nagliktiselo.

2. Naglingnerle, taimna Issumagivara

Naglimut Jêsuse Tokkungmat pivluta,
Illagelêrkovluta;

Nagliktigêkta,

Jêsuse illa

Naglingmat taimak.

\section{0\%. Mel. 79.}

$\mathrm{Ja}$ lass in der Gemeine. 520.

1. Jêsuse! illagêktut Nellunaikusilaukit, Nagligilaurangne; Ullapirsalaungmigit, NagliktigêKovlugit Kuviagijarnut mâne.

2. Naglingnernut illuvut Taimak ônarsilaulit Tækkuksaukovlugo, Piniarniptingnullo Arvertarniptingnullo Nekkorsijaulerkovluta.

\section{Mel. 97.}

Inukotitit illagêt Tagva avisuêrtikit; Tamaitale erkaitikit Kingorlermik perkojarnik Nagliktigêgungnailerkonnagit, AttausiutsainalerKovlugit. 
194 Ingminik illitarksivallianermik

INGMINIK ILLITARKSIVALLIANERMIK, ANNERSAUMINERMIGLO SAIMARTITAUJOMANERMUT.

\section{Mel. 15.}

Mein Heiland! Du verwirfst uns nicht. 522.

1. Jêsus ajæktoratalo

Erdligidlarpaptigut

Kuviasuktilutalo

Erkatsainarpaptigut.

2. Kina illiktut saimava Nâpkiniktorsôvlune? Kina kamangitsertorka Akkêngaijunik mâne?

3. Nellâgôrlune tâpsoma Nagligidlarpâtigut, Kiksartigalloartapta Allarungnaimattigut.

\section{Mel. 37.}

Wir sind vom Fluche frei. 523.

1. Uminajuêrtut

Kittorngaugapta

Gûmut, illuptigut

Ajudlarpogut

Sulle ajornivut

Uvlut tamaita

Pertaugiazarput

Jêsub aunganut.

2. Pitlaræksaunermik Pijungnailuta,
Sulle unnuktunnik

Nunamêdluta

Ajortokarpogut,

Gûdib sângane

Kiksautigijavut,

Kaut tamaitale.

\section{Mel. 214.}

Sündig bin und bleib' ich immer.. 524.

Ajorsatsainarpungale Aunga pigingikupko, Kannimatsainarpungale Aiparingikupkole; Jêsusib inûlijima Uviningminut illa Inûlijomavânga Idluinimnit tamainit, Jêsub âniaminut Ômatiga âkikpâ.

\section{Mel. 166.}

Senningajolingmêdlutit. Jêsus âniagavit, Ajorungnailerkovlunga Mattomanêtillunga; Ajornimnit ubvarminga. Aungnut kovimajomut : Illingne uvlut tamaita Kuviasukovlunga. 
annersauminermiglo saimartitaujomanermut. 195

\section{Mel. 218.}

Herr! öftne mir die Tiefe meiner Sünden. 526.

1. Nâlegar ! Kaujitilaungminga

Ajornimnik nâmaktomik; Taimagletauk tækkotiminga

Saimanivit angininganik: MerngoêrneKsitailitinga Igvit assingne, Nâlegara, Illingnele nagvârtinga, Okartotit: „Okumaitose „Ajornipsingnut, kaileritse

"Nekkorsititsomavapse.“

2. Saugârsuk pivianadlartor Johannesib tækkojanga Toкkotautillugo кejungme!

Pêjakittôk ajornerit Uvamnit, najulaungmingalo,

Kanoк illinganeKarnernik, Ôksimarilerkovlunga, Illikullo sâlakartinga Uviningmik, Sâtanasemik, Ajortuniglo tamainik.

\section{Mel. 165.}

Jesu, Brunnquell aller Gnaden. 528. 1. Jêsus nâpkigosuktotit, Ajæktolunginangne Kiksartor ajornerminik,
Nekkorsitigangnele: Attêtôk piulinga Ajornimnit, Gûdiga, Illungertornermut mânna Tuksiarapkit tussarminga.

2. Aggangnut tigumianga Majangadlaramale, Saimanerniglo tunninga Kappiasungnêjarlunga, Tessiulaungmingalo Illa nutarak sorlo, Sâtanasib pêrkonnanga Illingnit, Piulijiga.

\section{Mèl. 168.}

Entbinde mich mein Gott von allen Banden.

1. Gûdîk! kellarudsâlaunga Kellaktimnit tamainit, Uviningmut pititimnit Sulle mâne nuname. Kellaksimajuksauguma, Tagva ômatekulluga Kellaksoruk, illingnut Kivgautsainarkovlunga.

2. Kaujimavunga, Jêsuse Nagligigalloarapkit; Ikpigikassiudjivunga Nâmanginimnik sulle, Pitakarmallo illumne Ullapitseкattartomik Tamainit piulinga Pigimariklungalo! 
456. Mel. 132 a.

O Jesu Christe Gottes Sohn. 529.

1. Jêsuse Gûdib Erninga

Pingortitsijôjotit!

Takpânget tækkosalaunga,

Tussakit okautsikka:

Igvit pingortilauramga

Piulijigigapkillo

Ikkajutsainarminga.

2. Tækkonânga pingortitat, Nangmineк sennalauktat! Omavît puigorlunga Ajorpâ illapsumut. Aggaktit tækkosalaukit, Pivlunga kejuksoarmut Kikkiæktortaulauktut.

3. Tâpкonanele attera Aungnut aglaksimavok, Ikkit tækkokit, pivlunga Tâpkonanêtsainartut; Tagva ômavît nagliktub Nâpkigidlarniarpânga Nipportilârlungalo.

\section{5\%. Mel. 79.}

Herr Jesu, mach' mich selig. 831.

Idluarsilaungminga Ikkingnut Jêsusiga, Inûlinga aungnut Pivlunga tokkogavit,
Asserorsimanerit

Tokkotikit uvamnêtut.

458. Mel. 167.

O dass ich der Sünde sterben.580.

1. Kanoktôk idluitunut

Tokkojotun-ilanga

InôKovlunga Gûddimnut,

Tâpsoma âkiklinga.

Opiginersarilago

Pivlunga tokkolaungmat,

Ajorsaralloartunga,

Sulle nâpkigivânga.

2. Kanoktôrlo ômatiga Angmatsainarle Gûmut Jêsusiblo naglingninga

Najorlugo tarnipkut;

KanoKtôk tættigilago

Gûdiga illûnapkut,

Pairitsainarniarmanga

Pijariakarnimnut.

3. Kanoktôk arvertarlanga,

Kittorngangititullo,

Perkutaule inôsera

Gûmut, adsigilugo,

Napârtub akkerungatut

Tarniga iptekarle;

Ôma idluarijanganut

Inôkovlunga mâne. 
annersauminermiglo saimartitaujomanermut. 197

459. Mel. 79.

Wenn Du von Deinen Gaben. 531.

1. Nâlegar tunnijarnik Akkillêkojiguvit, ErKartuivingmut Pitikuptigut, tagva Ajortoliksôgapta Kioluta sapperpogut.

2. Taimaigalloartogut, Ajæktungilaptigut Kittorngariluta, Kakrialeruptale; Ômigitsænginangne Pingortitat tættigijit.

\section{0, Mel. 132 a.}

Du bist der Hirt, der Schwache trägt. 532 .

Sangêtut pairidlarangne Illingnut Jêsusiga Pivunga, inûligangne Kanoêtut tamaita; Nẻllâgut kanoênama Aungnut inûlilaungminga Tarniga pairilugo.

\section{Mel. 97.}

O Jesu, Du mein treuer Hirt. 533.

1. Jêsusiga pairijiga!

Piniutidlalaukparma

Pingnik pivianartunik Piloriktitsainartimnik :

Kanoktôk kaut tamât ômatima

Kujaginersarilitit mânna.
2. Kaujimagalloarpotit, Kiksautigikattartamnik: Ajungituinarlunga Ajoksarkattarpakkama ; Issumagijungnaivigilaunga

Nutakkatut majangadlarnimnik.

\section{Mel. 141.}

O ich armer Sünder. 534.

1. Ajoktoksôvunga

Nellâgut illa, Nâpkiniktub Jêsub Saimadlarlune, Nivioringikuninga Itsar tâpsoma Saimautsungnaipânga Ipperarlunga.

2. Kuviasuklunga Nerriukpunga, Allatsængimanga. Tættigivara, Erkaititsinine Saimarsautiggit Uvamnut tamaita Nâniarligit.

\section{Mel. 26.}

$\mathrm{Du}$ treuer Seelenbräutigam 535 .

1. Tarninik naglingniktotit Sulle agviarpakkapkille, 
Ômatît âniatikapko, Omigivunga uvamnik.

2. Ajornima miklitipânga, Piluartomik naglingnivît, Kiksartigalloarapkille Sulle, nâpkigênaramga.

3. Illingnik ungadlarnera Kuviasûtigidlarpara, Illungertornermut nellâgut

Nertorupkit inôsimnut.

\section{Mel. 228.}

Wie sind wir doch so herzlich schlecht. 538 .

1. Ajortoksôjogut illa, Jêsub nâpkigivâtigut Taiksaungitomik illa. Ôminga sillatunermut Uviningmut aungmullônêt Kaujititaungilagut, Gûdib anerninganulle, Tâmna missigivavulle Ôma kaujitimmattigut,

2. Kattangnera Gûb sângane Kangusuktigidlarpara, Koaksârutigilugo ; Ôma, Engelivaksuit Tuksiarvigigunitsuk, Mattujutigijangata. Sôgle Jêsub ajortunga
Naglikpângâ? Kaujivunga:

Tokkominut piulingmanga.

3. Tarnipkut kuviasukpunga Jêsusemut okperama, Ajorungnaitimmanga, Tâpsomalo nagliktima Aiparitsainalermanga Mâne inôtillunga; Kuvianak, illagênut Pitimmanga, nertorpara, Tâpkonanêtsainarlanga.

\section{Mel. 4.}

Gott heiliger Geist. 539.

1. Anerneк Gûde!

Nertorpagille, Kaujitinamga, Ajortolik Jêsub nâpkigingmago.

2. Kennuênermut, Saimarsainermut Tessiormanga ;

Tamanna ipaksijungnangilara.

3. Kugvioruma,

Piulijiga

Kujagivlugo,

Tagva ômatiga nekkoksijauvok. 
annersauminermiglo saimartitaujomanermut. 199

4. Ivsôgamale, Kangusukpunga, Uvamnut aungat

Piniarninga pituarivara.

5. Naglingningale, Angivor illa, Kêmitikame Tokkotigivlunga . aunâdlarlune.

\section{Mel. 141.}

Herr und Gott der Deinen. 540.

1. Nâlegar Gûdivut!

Pigiarmitit,

Keasinivullo

Tussalaungmigit;

SaimartilaukittôK

Timekulluvut,

Sajungaitikillo,

Illa tarnivut!

2. Inôsiptingnetôk

Illagittigut,

Idluarijarnut

Âriksortigut,

Illagêktokotitit

Missigitikit,

Najuênarnernik

Nagligosumut.

\section{6\%. Mel. 155.}

Neige Dich, Du naher Mann. 541. 1. Nâlegak! mannilaurit Kittorngarnut aklujomut;
Saimailutit,

Tækkosalaunga, illa

Mânnamit

Saimanivit angininga,

Mannigorutigilugo

Sangnêtsainarkovlunga.

2. Epkêjarvigilaunga

Piunginimnik tamainik,

Ubvalaunga

Aungnut ivsornaitomut;

Illagêt

Akkorngænne, pairilaunga

Illingnit tammarkonnanga Inôsimne illûnâne.

\section{Mel. 121.}

O mein Immanuel. 543.

1. Imanuêliga!

Nagliguk tarniga

Iktôdlarmat sângne,

Kugviolarmelo

Missigilaukârdl une:

Piulijima

Nâpkigingmanga,

Ajortôvlunga.

2. Jêsuse! tarniga

Saimarsaruk mânna,

Pitsartutilugo,

Aungnut serparsaruk,

Okarvigilungalo:

Pivakka pitit 
Pikka pivattit,

Pilorikpotit.

3. Inuit nagliktut

Kilangmiut aglât,

Kattinajarunik,

Tâpkoa naglingningit

Amigarput sulle

Nagliktiptingnut,

Naglingningale

Anginerôvor.

4. Jêsus saimarsainît

Missigitsainarlavut!

Tarnivut nâpkigikit

Tækkonâkit kugvivut;

Kennuvigidlarlutit

Tikkipaptigit,

Uvaptingnit pêkit

Perkungitatit.

5. Okpernartôjotit, Nagliktotôjotit

Gûd! nâpkigigamga.

Ajortunga tamât

Tættetuakartunga

Jêsús illingnik,

Pigilaungminga

Illûnarmale.

\section{Mel. 97.}

Mit einem tiefgebeugten Sinn. 544.

1. Tarnipkut, kibliktaurlunga

Gûb sângane pâmakpunga,
Kugviorlunga serrrivunga, Tâpsoma tessiormanga; Inûlernimnit tagvunga aglât,

Saimaninga uvamnit pêng-imat.

2. Nellopkotivunga tagva, Angidlarmat saimaninga Kangusudlarpunga illa Næglingnekitsainarama; Ôma piniarningit aungalo. Okpernima pitsartutigivait.

3. Kujanak Kennilaungmanga

Nagvârlungalo pingmanga ;

Kujanak kaujimagapko, Tâpsomalo pigingmanga; Kujanak, nellâgôrvigingInanga

Taimaimat tâpsomanênarniarpunga.

480. Mel. 30.

Ach mein Heiland, segne meine Seele. 545 .

1. Jêsuse! tarniga nâpkigiuk,

Illumne Kêmitiktor illingnut,

Ômattivîlle

Najorninganênaromagame. 
annersauminermiglo saimartitaujomanermut. 201

2. Anernerpit innerteriningit Nippingalo nâlelaungikup. kit

Ômame, tagva

Kiksarnermik ikpigidlarpunga.

3. Jêsusiga Kugviortiminga, Tappagilerupkille Gûdiga, Ama nutâmik

Missigitiminga saimanernik.

\section{Mel. 146.}

Der Herr sah Petrum an. 546.

1. Nâlekab Pêtruse Tækkosarmago, tagva Aumadlalerlune Inukangitomullo Ailerpok, tagvane Ogguartok Gûmut; Tammadlarninelo Keajutigivâ.

2. Kamatsiaritse! Jêsusib ijikita Innertermassele Ajornipsingnik illa. Ajungitsuginek Pitlalermagole, Kakkialertulle Ajorningit pêrpait.

3. Nakudlarpok mânna, Tukkisititaugapta
Tarniptingne Jêsub

Ullapirsaininganik;

Kuviasukpogut

Najorangatigut,

Nagligæksaunata

Illannâringmattigut.

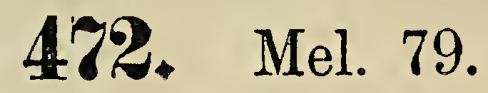

Mit einem tiefen Sehnen. 548.

1. Tarnipkut kippipunga

Kajungilârlungalo

Tækkonârtaunimnik,

Ômingalo piguma,

Nutâmik inôvunga,

Tamainit angnerpângongmat.

2. Tokkojotun-ipunga Asserorsimavlunga Illûnapkut, Jêsus Piginæk; taimaimalle Ikkingne nagvârlago Inôgutiksar tarnimnut.

3. Taiksaungitomik mânna Nagiigitsainaramga Kollaromananga, Nellâgut pigivagit Pigivarmalo igvit; Saimalaunga Nâlegara!

\section{2 a. Mel. 167.}

Heilger Geist voll Gnad und Liebe. 549.

Anerner naglingniktotit, Kennuvigidlarpagit: 
Innertinga, kiksartikupkit,

Pêjaivigisârlunga!

Piniaromadlartamnik Angerkattalauruma Angernikka serkomilugit Ômamut kiksarpunga.

\section{Mel. 221.}

Freundlichster Jesu! ich hoff'. 553.

1. Pitsiarnerpaujor

Nâlegar Jêsuse!

Saimanermik nerriuk-

$$
\text { punga, }
$$

Kênat saimarsaijox manniuk uvamnut

Tarnigalo innigiuk!

Illupkullo pitsartuti-

$$
\text { launga, }
$$

Nellâgut illingnênarkovlunga ;

Ômattiga eprêjaruk, inûlinga,

Nellâgullo perkutigitsainalaunga.

2. Takopsoaginerpângojor Jêsuse!

Tussakittôk rennunikka, Tækkolaurit каnок tarniga kêdlarmat!

Akкeartortitsuk illingnik! Itsak nunamêdlutit inuit Perlilerkojomalaunginangne,
Tamanna pivlugo, naglingniojotit

Tarniga kâdlartor kamagilârivat.

\section{4\%. Mel. 30 .}

Lieher Heiland, blick' mich an. 556. 1. Piulijîk tækkonâlaungminga

Attaruitilunga nutâmik, Kenuêviginga!

Idluinikkalo pêjalaukit.

2. KanoKtôk aumalerlanga sângne!

Tamainillo nagliluarlagit!

Nâmagijarnut

Axiktaunajarpungale, tagva.

\section{Mel. 79.}

Nimm mich mit Lieb'serbarmen. *774.

Aggangnut tigulaunga Pairiuk ômatiga, Pallangaertilauruk, Ullapitaukonnago Nangianadlartunut, Illingnulle angmalerle.

\section{Mel. 195.}

Unbeschreiblich's Herze. 558.

1. Nellekangitotit Gûd, sakkerviginga 
annersauminermiglo saimartitaujomanermut. 203

Illingnik ikpigilanga!

Missigingikupkit

Inôtsianginama;

Taimaimalle Kaitaulaule

Tarnima piksanga;

Nekkorsitiksamnik

Illumertaulanga.

2. Urlomelo sulle

Tussarlago nippît,

Pivlungataur toKKogavit;

Angervigivagit;

Igvit tessiunga!

Pêjakillo ajornikka

Nutâmik, tamarmik

Time tarne pikik,

Aukpit akkiksagik.

\section{8\%. Mel. $151 \mathrm{i}$.}

Ein armes Menschenherze.

1. Inukullub ômattâ

Ujarkatun-itok

Jêsub ânianingat

Aggilitilerpâ ;

Aungat mitsuktimmago

Opigosalerpok,

Jêsusib adsingannt,

Pingortitaulerpok.

2. Tamaita ajornivut,

Piulijiptingnut

Okautigiguptigik,

Nâpkigivâtigut;

Tâmna avatiptingnik

Attudlartor sulle,
Inûlijiksakarpoк

Inônartolingmik.

3. Taimak kannimakôrtut Aungminut saimarpait, Tamaita tuksiartut Axiktaujomamut, Sânganelo Keajut Mannigudlalerpait, Kannimakôrningillo Pijungnaitilerpait.

\section{Mel. 22.}

Ich finde mehr als ein Versehn. 559.

1. Illa tammarkattarama,

Taimak ikpigilerpara:

Ajortokullôdlarama

Ittiggangnutpâmakpunga.

2. Jêsuse tuksiarpagit Ama ubvarminga aungnut, Umunga âkilaungminga, Sângne idluarkovlunga.

\section{Mel. 146.}

Ich armes Würmlein bin. 560 .

1. Nellâgut illumne Ajortoliksôvunga, Jêsuble aungminut Pissiarilaungmanga; Aivigilerpara Illunarsoamnut, Ikkit kunnikpakka Annerilaungmanga. 
204 Ingminik illitarksivallianermik etc.

2. Nagliktiga mânna Tarnima ungagivâ, Tokkotigingmanga Inôtsainarkovlunga; Mannigorsârpânga IllannâKarnermik Piksakartilunga Nungusuitomik.

3. Nâma gijanganut Inôtsianginama Kênangat sângane Illerasudlarpunga, Saimarsautib nippâa Tussaromavara, Nâlengniarnimnik Kennuvigivara.

4. Ikpigingikuma Asserorsimanimnik, Jêsublo ômatâ Angmangipat uvamnut, Inônerle mâne Ajudlarivara,

Nâksaungitomullo A ssiolerlunga.

5. Tagva Nâlegara,

Kujagiviktaulerle!

Taimak pitingmanga

Nâpkiningnermut mâne; Inôtigigapko

Pinnanga uvamnik, Jêsusible aunga

Nâmaktôngmet Gûmut.
6. Saugârsuk opinak

Kujagivagit illa!

Kaikogamga augnut

Kovijomut pivlunga;

Tagva âniaruut

Piginga Gûdiga!

Mâne illanâmut

Nâkssungitomullo.

\section{Mel. 218.}

O Jesu, Du mein Seligmacher.

1. Jêsuse piloriktitiga!

Allarkattaralloartagit Ipperalautsængilarmale, SuKKosingimat pairksinît; Erkisimiarkattaramga Saimarlunga, oxarpakpunga:

Kina illiktun-inerka? AjoKsartut nellungilattit, Aklujulle pillisarpattit; Taimainama nâpkiginga!

2. Naglingniktor Piulijiga Nertutsainarlagit illa! Ikkajutsainalaungikumga Nanênajarkîk mânnakut? Inôserlungnadlartomulle Nangianartoliksoarme Asserorajarpungale ; Kanoêkumale pairinga, Angergarviksamnut pitinga

Najutsainalaungmingalo. 


\section{IVSORNAITITSINERMIK TIMEMIGLO TARNE- MIGLO.}

\section{Mel. 79.}

1. Ômatimnêtsainarlit, Tokкotab Nâlegapta Ikkingit aungalo; Kaumartigidlarapkit, Nekkorsitingmangalo, Kuviasuktimmangalo.

2. Taimaimat, Jêsub aungat, AjortoKsôniptingnit Âkiksorpâtigut; Piksaringikuptigo Missigijungnailugo ToKkungajotun-ipogut.

3. Aggut kovilaurangne Sujungnerit uvamne Pêjatsialaukit!

Nagligosulertinga! Kuviasukovlunga Illingne, inôtillunga.

4. Kiglissiniaruma, Uvamnik, nagvârpunga, Ajortoksôgama, Nachonadlaramalo; Nâlekable tokкunga Piulijiksarivara.

\section{Mel. 149.}

Selig ist ein reines Herz. 562.

1. Idluarsivok ômat Jêsub ikkinginut
ToKkunganut aunganut Sujungaititaujok ; Jêsusib ômattâ

Illeraginago

Tækkosarungnarpâ.

2. Kanortôk Jêsusiga

Nagligênarlagit,

Nâksaungitomut illa

Kêmitigilutit;

Assingnik illale

Kângnangale mâne,

Missigilagittôk!

\section{Mel. 151 a.}

Du, dessen menschlich Leben. 563.

Jêsuse, uviningme

Inôtsît illûnât,

Inôsipta nuname

Âkiksûtigivât;

Tokkogavit pivluta

Mânna inôvogut;

Aungnut ubvartaugapta

Idluarsivogut.

\section{Mel. 22.}

Nimm Dich, Du theurer Schmerzensmann. ${ }^{*} 789$.

1. Jêsuse âniajotit,

Tarnivut nâppkigilaukit,

Timiptingnut tarniptingnut

Attît nertutsainarlavut. 
2. Kaut tamât sapputitigut Perkonnata ajortomut; Taimak inôsek nuname. Ivsornaikovlugo sângne.

\section{Mel. 106.}

O Herr! gib meiner Seele Leben. 564.

1. Nâlegar! inôsekartiuk Tarniga, amuaruglo Illingnut : tunnidlalaukpotit

Pivlugo illa tokкomut, Illingnut perkutauvlune Ivsornaitsainarkovlugo.

2. Nâlegak! inôsekartiuk Tarniga, nutautingalo ; Ikkajorlunga ajorpunga Igville sappingilatit; Sangênama illûnapkut Ikkajulaunga Nâlegar.

3. Nâlegak inôsekartinga Pitingalo anernernik; Nippingatailitilaungalo Sunamut nâmangitomut, Kanôktok sulle nuname Anernekattigilagit!

\section{5 a. Mel. 230.}

Heiligster Jesu, Heil'gungquelle. 70.

1. Jêsuse ivsornaitotit Inungnullo ajortolingnut
Ivsornailijutaujotit!

Ômanartomik immermik, Mamartomik, issungitomik

Puilaviodlartotit!

Malligæksaugavit,

Adsiotilaunga

Illingnuttôk!

Jêsusiga, ikajunga,

Illiktut ivsornailanga!

2. Jêsuse nâlainartotit Atâtab pijomajanganik Tokкo aglât tikkidlugo! Taimaktauk pijomanera Sângne serkomitsainalaurle,

Pijomajat nâleklago! Sorusiarsutut

Nâletsiartotut Itilaunga! Jêsusiga, ikajunga, Illiktut nâlainarlanga!

\section{Mel. 155.}

Theuer Freund, hier ist mein Herz. 571.

1. Illænnar ômattiga

Perkovara naglingnernut,

Unganermut,

Ivsornarkonnagole

Nuname,

Kamaginagit assitit, Ônarsile naglingnernut Sakkijârtilauktarnut. 
2. Kittorngaritsianga, Ivsornaitiuk inôsek, Ikligunek

Nallâgut tokкolugo, Aungnullo

Asserorsimanimnille, Illupkut ubvartauvlunga, Nutautinga Gûdiga!

3. Gûd Anernek, piginga Tarnigalo pairilugo, Torkorlugo,

Sapperapko nangminer Pairinek :

Igville tessiorumga, Pitsartutilerumgalo, ÂkilârpoK inôsek.

\section{Mel. 22 a.}

Die Seele Christi heil'ge mich. 566.

1. Jêsuse tarningnut mânna Idluarsaruk tarniga, Illingnik Piulijiga Anernekattekarlanga!

2. Timingnullo ikkilingmut, Pivlunga aungêrotomut, Inûlidlakit ukkoa Tarnigalo timigalo.

3. Sennerkakut kappingmattit,

Kallugitsab inninganit, Aub immiublo kôdlartub Tarniga epkêjarliuk!
4. Aungmiglo kidsijârnernut Piulinga ajortunit, Âniarnut tokKungnullo Illupkut pitsartutinga.

5. Jêsuse tussalaungminga Ikkingnullo pullầrlanga, Torkortautsainarkovlunga. Ajortomut perkonnanga.

\section{Mel. 16.}

Liebe hat ihn her getrieben. 569 .

1. Naglingniub akkartipa Killangmit Gûb Erninga, Tokkotaungmallo pivlunga

Nagligæksarivara.

2. Naglingner kajungernernut

Ômatiga ônarle, Niglilerpallo, mânnakut Illumertaulermile.

3. Ajortorlo illumnêtor Toкkotilaungmiugle. Taimak idluarijarnut Âritsainarmingale.

4. Jêsusiga, piulinga! Sivorlimnit pijamnit, Idluitunit tamainit Sujungaitinga aungnut.

5. Kristuse saimarsainernut Tessiortigut taimak, 
Okperniptingnik illingnut Sagvaitsainarkovluta.

6. Ômativut issuanut Soккоsijuitikit, Perkojattit uvaptingnut Malliktaudlarkovlugit.

\section{Mel. 56.}

Segne, segne uns aus freiem Trieb. 576.

1. Saimartigut:,: Piulijivut Najorluta:,: naglingnernulle!

Nâlegar aunâravit Okpertunut tamainut! Ômativut:,:

Sângnêtsainarlit.

2. Inôtsivut:;: âkiksolauruk, Pairitigut:,: tammarkonata,

Serngnigitsainartigut, Ivsornailerkovluta, Kuviagi-jarnullotôK Nertorlaptigit!

\section{Mel. 16.}

Gib uns in den Gnadentagen. 577.

1. Saimanartovingme mâne Issumakartittigut Mallitsainaromavilutit Timekut tarnekullo.
2. Jêsuse aungnut tamapta,

Ubvatsainartiguttôk, Sângne idluarkovluta, Tamât inôsiptingne.

\section{Mel. 16.}

König, dem wir alle dienen.

1. Illingnik Gûde tamapta Kivgartorpogut mâne Nellâgulle pingmangâpta, Kaujimavat Jêsuse.

2. Issuma nautsertolauruk Nellâgut sâtermangât? Ungangmangâlle illingnut Takkomôrmangâllônêt?

3. Time tarne pairivâkka Kivgartorvmavlugo Inôserlo sapkupara Jêsusiga pivlugo.

\section{Mel. 185.}

Deinem Bilde ähnlicher zu werden. *Anh. 137.

Ablatsângortitaujomavlunga

Adsingnut, Jêsusiga!

Tarnima kajungerutigivâ

Kugviortipângalo

KanoKtôK nertornautiksarnullo

Idluarijarnullo ilanga! 
Arvertarnimne, tagva $\mathrm{Ku}$ viasugajarpunga.

\section{Mel. 37.}

Mach' uns're Herzen rein. *Anh. 140.

Aungnut ômattivut

Ivsornaitikit

AdseKarKovluta illûnaptigut

Illingnik Jêsuse.

Pigittigullo

Arvertartiluta

Tumingne mâne.

\section{Mel. 126.}

Wir 'r ennen nur die eine. $* 814$.

IvsornaitisimaneK

NelâgôrtotôjoK

Idluarijaujorlo

Atâtab sângane

Saugârsub aunganit pivor,

Tâmna nâmagivavut

Issokangitomut. .

\section{Mel. 166.}

Lass Du mich bei der Sünderschaft. 586.

Ajorama Anernernut Nekkorsitilaungminga, Gûdib nâmagijanganut
Inôsekarkovlunga,

Tarnigalo timigalo

Tamarmik pigilaukik,

Taimak opigidlarlutit

Kennuvigidlarpagit.

\section{Mel. 14.}

Den tiefen Eindruck, was. 589.

1. Jêsusib âniaminut

Piulilaungmanga;

Ômatingat naglingninga

Najoromavara.

2. Inukangitomêkuma,

Inutôtillunga

Jêsuse tuksiarupko,

Ônarpor illuga.

3. Aklujokullôdlarama

Nunamêtillunga,

Piulijiga najorlugo

Ungagênarpara.

4. Ômattiga kealerpat

Ôma najorliuk!

Akkitojomut aungminut Serparsariliuk !

5. Taimak nellopkotijunga

Ôma naglingmanga, Mallitsainaromavara Angergarkârnanga. 


\section{KRISTUSEMUT OKPERNERMIK ISSUMALINGA- SUERRERMIK.}

19\%. Mel. 208.

Wär'n wir nur stets Kinder. 590.

Kanoktôk! uvagut

NutakKatut Gûmut

Itsainalaukta!

Kuvianarnersoak

Pâne pijomârtoK

Ôgomavlugo,

Taimak inôseK mâne

Okumaisuêrtok, illa

Sulle inôvluta.

498. Mel. 184 a.

O süsser Stand, o selig's Leben. 591.

1. Inôser piloringnadlarmêk,

Pilertok miksekârtomit

Issumalingasuêrnermit,

Tungajorlo Kristusemut;

Ômat tagva alliangilar

Kristub aungat assianik;

Kêmitingnermulle rilangmik

Sâlakadlarpor tamainik.

2. Issumalingasuêrnerle Nênilauruk ômattimnut, Pêjakit assingit tamaita, Ivleridlarupkillônêt, Uigasuit sillatojut
Pigarkattigilaulakka, Tikkiviksat nerriugivlugo, Pijarêtsainarkovlunga.

\section{Mel. 165.}

Mein holdester Jesu. 592.

1. Ânanaunerpângojotit, Jêsus nellâgortunut, Naglingnadlartor ômatît. Sullele nâmaktomik, Illitaringilara;

Igville ikkajunga

Uvinik tokkolerlugo

IllingnînaK Kâkovlunga.

2. Illingne pitakadlarpoк

Kuvianadlartunik,

Serrinartut uviningmut

Uivêriklerpakput;

Nukkingêrutitipait

Okpernek næglingnerlo.

Illingnut Jêsus attajor

Kuviasudlarungnarpoк.

3. Illanârnerpângovotit

Tamainit Jêsus illa,

Ômatît aumatsainarpor

Naglimut uvaptingnik;

Tunnikassiutivat

Nâlegaunit illangnut 
Tagvane soraijunatik Kuviasudlarkovlugit.

4. Taimak tagva amulaunga Jêsusiga illingnut; Soraitiuk issumama Attanniotininga. Aukpit reblidlarningat Kaumaivigênarlinga, Kissiville Nâlegara Perkutigitsainalaunga.

\section{Mel. 37.}

Das wahre Christentum. 594.

1. Okpernek Jesumut

Nellâgôrune

Okidlarpok illa

Jêsub nangminek

Okumainingale

Pêjalaungmago:

'Tokkungmat pivluta,

Pijaukonnata,

\section{Orumaijutitit}

Gûmit pingilat

Kunnujorsônivit

Taimak pivâtit,

Ivleringikungne

Idluinitit

Jêsusivit tagva,

Pêjarniarpait.

3. Taimak kissiane

Pinialerit,

Ajoksarnerniglo
Ikpigiguvit

Jêsusib sânganut

Sakkijâlerit:

Ikkajudlarmattit,

Taimak piguvit.

4. Sorairnægle Gûde

Tættigigungne

Kaumajub apkutâ

Tækkoniarpat,

Tukkisilutillo

Okperijarnik,

Tunniniarmattit

Pijomajarnik.

\section{Mel. 228.}

Lamm, gieb uns das notwend'ge Stück. 600.

Saugârsuk · Gûd! tunnilauPijarialik uvaptingnut, ToKkut tautukôrlugo. Tâpsoma kibliklitigut, Mamaigijatit pêrlugit; Kajungertidlarligit Ômativut, Tussarnermut kissianut, Perkonnata, Piksarsimarerkovlutale.

\section{Mel. 16.}

Heil'ge Einfalt, Gnadenwunder. 603.

1. Issumalingasuernek

Gûdib tunnergutinga 
Sillatuneliksôvlune Pitsartunekadlarpoк.

2. Issumalingajor inuk Tamât rellaksortaungmat, Ânanaunek, akluineк Tættiginatsængilak.

3. Issumalingajuernermik Illumertauguptale, Tarniptingne kaumalerpoK

Tâksoarlo nipракрок.

4. Issumalingajuêrnek Jêsusib ikkinginit Puailavok, tâpkonane Nagvârtautuinarpok.

כ. Illa tættetuakartor Jêsusemik nagvârpoK Kairtomik tunganartomik, Gûblo kittorngarivâ.

\section{Mel. 14.}

Der Hohe und Erhabene. 604.

1. Kotsingnerub kittorngane Erdligitsainarpait, Nikkanartut illumingne Kuviagênarpait.
2. Tarnîk tamaita allakit Gûb perkungitangit, Nungusuitor kissiat Piniaruk illa.

3. Kennertiga Jêsusiga! Tessiulaungminga! Illautilauruk attera Kittorngarnut mânna.

\section{Mel. 165.}

Wenn doch alle Seelen wüssten. 606 .

1. Kanortôk Kaujimalerlit Inuit illûnatik, KanoK piloringninginik Okpertut nellâgôrtut. Illa piloringnerme Sivumuatsainarput; Inôtsib apkutânêput Saimaneksitsainarpullo.

2. Kanoktôk Jêsub tamapta Pigitsainarlittigut! Pitsartunekanginapta Pitsartutilitigut! Sâtikit issumavut Jêsusiga illingnut, Tagva miksekârnernêpogut

Ijivullo kaumaudsauvut. 


\section{ÔMATIB AKLUNINGANIK NELLOPKOTINERMIGLO.}

\section{Mel. 165.}

Meine Armut macht mich schreien. 608.

1. Aklunima Jêsusiga

Kaigarsudlartipânga

Illingnut saimarsaijotit,

Piksanartitigalo;

Igviovotit illa

Issumagênartara,

Mâne Kugvioraiguma,

Omatit aumavor tagva.

2. Nâpkigosuklutit illa

Pagreatsainarparma,

Akludlartunga uvanga

Erkisimiarparma :

Kêmitingnera illa

Taimaktauk kugvinikka,

Kaujimagangne Jêsuse,

Serritipat ômatiga.

3. Ah âk tarniga Kêdlartok Kaivok puailavingnut, Ki ppinermut Kannimavok, Nekkoksititsungnarpat, Ill ummertilungalo $\mathrm{Ku}$ viasûtiksamnik Ak keartortiksamniglo Ajoksarungnaerkovlunga.

\section{Mel. 121.}

Ach, was ich bin und thu'. 614 .

Kanoêleruma,

Sapputingikumga,
Illingnit pijakka

Ajungitut ama

Kattagajârivakka;

Piulijiga

Najuênanga,

Ajoksarama.

\section{0\%. Mel. $151 \mathrm{i}$.}

Ach, ohne Jesu Liebe. 615.

1. Jêsusib naglingninga

KaumaniôdlartoK

Piginago, uvanga

Kajungernikkalo,

Sunautsængilat illa:

Taimak tarnekulluk

Niplerpor Jêsusemut

Piulijiminut.

2. Piniaromagune

Ajungitomigle

Tagva nipliadlarpoк

Jêsumut, tâmnalo

Ômane piniarpok:

Tarniblo tagvale

Ômamut kujagivâ,

Ikkajulauktine.

\section{Mel. 22. a.}

Mein Heiland, dass ich ohne

Dich. 617 .

1. Pituarigapkit Gûde!

Uvamnut piniarnîlle 
Tamât kuviagigapko

Opigomut pâmakpunga.

2. Ajortunga pigivarma, Opinak taimaitinamga; Missigigapko, nellâgut Najorangne ajortuggit.

3. Sulle piojorigama

Okumaitsainalaukpunga; Miklilertilaukârdlunga Ômatipkut okipunga.

4. Taimaitsainarkovlungale Jêsus, pigannertiminga ; Tamât pituarilagit NangmineKatsænginama.

\section{Mel. 185.}

Lasst uns ihm ein Halleluja singen. 620 .

1. Hallêlujamik tuksialaukta Piulijaudlarapta!

Ivsornainermiglo piniarta Tunnergutaulutalo;

Aggakpit issamijut inungnut

Tigulutalo eriilittigut,

OKaravit naglimut

Kailauritse uvamnut.

2. Kinaulaukpitâ? sullelo mânna

Illitarivaptigut;
Nagligosumut kenneraptigut,

Ajulidlartilluta;

Jêsuse, âniarnik ikkingnik

Kejungme pijarnik, sakkêgavit

Okpertunut nellînut, Tagva ônarsilerput.

3. Aklunima angijorsôninga Okautaujungnangilak, Jêsuble aungminut saimarmanga

Mannigudlarivânga.

Timît torkojok tautulaulago,

NekkoKsilaungminga ikkingnullo,

Mâne inôtillunga Kuviasukovlunga.

4. Napkiningat angijorsôninga

Ipaksinatsængilak,

Uvaptingnut, ajortoksôluta

Illannârimut taimak:

Ôma ômattâ kêmitilermat Saimaromavluta naglingnermut;

Tuksiudsininganut

Idluarsilerpogut. 
SOKKOSIJUINERMIGLO SAIMARTITAUVALLIANERMIGLO.

510. Mel. 217.

Wer durch das Opfer Jesu Christ. 621.

1. Jêsub tunnergutauninganut

Ajornekarungnaîtitaujor, Ômamut Kêmitigivor Attavigênaromavlugo; Ômaterartitaukovor Nutâmik, illumertaujomik Kujalinermik, naglingmiglo

Tâpsoma ânianinginut, Taimaglo sakkêtsainar. por

Perkutaugame Jêsumut.

2. Illinganek ânanauvok Jêsuse okpervigilugo, Tâpsomalo agganginut Pairijautsainarlune mâne. Tagva inuk serridlarpok, Issumalisuêrlunelo,

KemâvoK uvingub pinginik

Pinasuarlune Jêsusib Adsinganik taimaiporlo Jêsub pitsartutingmago.

\section{Mel. 29.}

Hier legt mein Sinn sich. 623.

1. Sângnut Jêsuse pâmakpunga
Tarnima kaikovâtit ama: Naglimut tækkosalauruk! Aklunera nâpkigiuk.

2. Asserorsimanersoara Tokkungnut torkotaule illa!

Naglingneralo uvamnik Ipititaule âniarnut.

3. Missigigalloarpungalo Naglikapkit nâleklutillo Illingnut sulle naglingneк Nellâgut âkigêngilar.

4. Sulle nunamêtillungale Epkêjariakarpungale, Anernerpit issumaga Âziluariakarpâ.

5. Illingnêtsainaromavunga Assingnullo sangukonanga,

Sôngotipat arvertarnera, Aprome pâlakounanga.

6. Naglingnît illitarivara, Nellâgốrvigitsainaramga; Allarungnaiparma illa Pijungnaititaukârnanga.

7. Tarnimale illinganinga Illingnut pitênalerpara Kollarungnaitilaungminga Illingnut piulijaunimnik. 
512. Mel. 97.

Herr Jesu, der Du uas bestellt. 625 .

Nâlegak perкogaptigut,

Mâne sakkêjuksaugapta

Illingnut perorsianik

Anernernillo pijunik.

Tagva pitsartutilaungmittigut

Sagvaijuitokalerkonnago.

513. Mel. 10.

Ach lass auf Dein Versühnen. 626.

1. Jêsusele illingnut

Tamap ta kennuvogut,

Ômatti vut nippilit,

Aungn ullo âniarnut.

2. Aglisitsainarluta

Inôniarkovluta

Idluarijarnulle,

Tamât inôsiptingne.

\section{Mel. 167.}

Gnade, die aus Jesu Wunden. 629.

1. Saimanerub kôtsainartub Jêsusib ikkingnit, Akkerkutut napârtomut Illagêktipâtigut; Nangminek ajoraptigo Parritsainarpâtigut Inôsertârtilutalo Agviartut pêrlugit.
2. Jêsusib ânianganut

Kibliktaulerangapta, Inôniarnek serrimut

Pivavut tâpsomunga,

Piulijigigaptigo ;

Issuma, piniarnek

Naglingnermut pivavullo

Naglingmattigut taimak.

\section{Mel. 232.}

Ich halte mich getrost zu Dir. 630 .

Najuênaromavagit

Najormigamgatauk igvit,

Tagga kuviasûtiga;

Kairtotun-itôgavillo,

Tamanna tættigigapko

Tungavigênalerpara;

Tættigivâtit tarnima,

Ungagællutit Jêsuse

Tækkôterkâranuk aglât,

Piviksara nelliupat,

Nâlegara

Jêsuse nerriukpunga, lllingnut angergartinga.

\section{Mel. 214.}

Bis an's Ende unserer Tage. 631.

1. Uvluvut nâkârtinnagit

Merngoêrkârtinnata, Apperiakadlarpogut Akkulaitomik illa, Piulijipta sângane: Têppsoma saimaninga Tunnergutingillotauk Tunnergusearijavut. 
Nertornautânut tamât Attormangâptigiktauk?

2. Tagva kiksarnermut kugvit

Kugviorutaujôngovut, Missigigapta nellâgut Sunaunajarniptingnik Ôma saimatsainarnermut Pairilaungipattigut, Apkuta miksekârtor Tamaigijaulerkonnago, Tagvalo opigomut Nutâmik angerpavut.

\section{1\%. Mel. 115.}

Nun Kindlein bleibt. 632 .

1. Kittorngat Jêsusemênaritsetôk!
Tarnib pillorijutigijanga Attavigitsainarsiuk tamâne!

Errissiuk pivianartotut; Ahâk pivluta tokkomut Næglingnermut aivoK; Ungagissiuktôk!

2. Kittorngat Jêsub ikkinginêtitse

Tamanna kamagitsainarsiuk,

Tagvunga kivitisigik tarnise,

Tagva serrijutiksarsivose Illa soraijuitomik, Tamâne nuname Nâksaungitomelo.

\section{KIKSARNARTOME KENUERSÂRNERMIGLO MANNI- GORNERMIGLO.}

518. Mel. 83.

Mein Erlöser kennet mich. 633.

1. Jêsusima Kaujivæit Kugvikka serrineralo; Ômatîgle taimaimat Pairijit ungagênaruk; Saukane kaujingmagit Taigivaillo attingit.

2. Naglingninga angivok! Illagijomangmattigut, Ikpigikattaumingmat
Kiksarniptingnik tamainik,

Uvinikattigêmut

Kemangilâtigullo.

5̄19. Mel. 22.

Jesu! Du edler Bräut'gam. 634.

1. Jêsuse ivlerijara!

Tattigivâtit tarnima

Kuviagitsainarpagit

Tamainit angnerpaujomik. 
218 Kiksarnartome kennuêrsârnermiglo

2. Jêsuse erkarangapkit Kissivit tættigivlutit, Ômattiga serrilerpok, Illûnara nekkoksivok.

3. Kimut sânajartuksauvîk? Pingikumale illingnut, Illa Jêsuse, illingne Nâmaksivunga tamaine.

4. Piuligamga tokkungnut Kiksarnartunit tamainit; Illûnarma pigigamga, Kinamut akKonartauvîk?

5. Okperlune naglingniktor Nellâgut tukkisilerpor: Jêsusemut tættekartut, Tamaine ikkajortauvut.

6. Timipkut tarnipkullônêt Okumaikuma sunamut, Jêsuse tættigivagit, Piuligamga tamainit.

7. Kemangnanga oKaravit, OKautsit sokrosingilat, Nâksaungitomullo aglât, Pairijigigapkit tamât.

8. Jêsusemik pekangitor Ômamine tokKungâvor; Tâpsomingale pekartok, Serrivlunele inôvok.

\section{อ̆19 ̊. Mel. 16.}

Gott wills machen, dass die Sachen. 636:

1. Gûb illingatitsiarpait Tukkisiniartatit;
Mallît unêt iktulijârlît, Jêsumêtuinaruvit.

2. Idluarkutiksainarnik Pilâravit, okperit; Nipporuvit ômatikkut Siarklerungnaerpotit.

3. Gûb aggangit pitsiarput Tamaita pijungnarpait; Okumaitut nangianartut Sappiutitsængilait.

4. Gûd piviksakarkojikpat Ikajortaulârpotit, Suingartaunernut aglât Kangusuktitauvlutit.

\section{Mel. $151 \mathrm{i}$.}

Treulich will ich Gott bitten. 639 .

1. Gud Kennuvigivara

Tættigilugolo,

Siornioleruma

Nangiarnartune;

Allaromangilânga,

Nâpkiniktub Jêsub,

Illa najulerpânga

Siornioruma.

2. Jêsus pitilaukpara Omattimnut illa; Aunelo kovilaukpâ Uvanga pivlunga, Piulijaukovlunga Âniaviksamnit; Illale naglingninga Nellekatsængilar. 
3. Aut, kovijok pivlugo Nâlegar piginga!

Missigigaplio tagva

Kuviasukpunga;

Mâne inôtillunga

Okpervigigapkit

Piulijiga pâne

Tækkojomârpagit.

\section{Mel. 14.}

1. Ungagigapkit, assitit Kammagingilakka, Jêsus kuviagigapkit. Allaromavakka.

2. Ovane okperigapkit, Inôtigivlutit;

Pâne tækkojomârpagit Nakoridlarlutit.

\section{Mel. 164.}

Zuletzt gehts wol. 640 .

1. Kingorlermik tâmna illa, Idlualauktor mâne Jêsub Kristusib aunganut, Inôtsiaromârpok:

Jâr Kuvianartok

Piulijaunartok.

Kingorlermik kaijomârpoк Kuviasugvigijavut.

2. Kingorlermik Gûdib pine Mâne ôktorsimajut, Nâlegauvingminut illa Illallioromârpait Adsezartidlarpait
Gûb Engelinginik;

Sivornganele aikovait Kugviniglo perartipait.

3. Kingorlermik, ômattîgle, Tamanna kamagiuk! Tâmnalo kiksadlartitit Naglixassiutitille Najutuinaruk Issumakarlutit: Gûdib pijomajanganut Illinganera taimaipoк.

\section{Mel. 160.}

Was Gott thut, das ist wol. 641 .

1. Gûb sullijangit nâmakput, Issumangit idluarput: Kanorlônêt pilerangat Nâlegomadlarpara.

Tâpsomale - Pairijima Serngnigijungnarmanga, Ômunga ungavunga.

2. Gûb sullijangit nâmakput, Piulijaukovângı

Kaumaksarlungalo aglât,

Pigitsainadlarpânga; Naglimullo pigigupko, Sakkijalerpor tagva, MikseKârrtongmat tâmna.

\section{Mel. 155.}

Du bist ja mein Licht und Stern. 644 .

1. Inôsungnimnit aglât Gûde, kaumaksalaukparma 
Errilunga;

Inôsimnelo tamât

Pairksinît

Tukkisigapko, ômamut

Kujalimut ittiggangnut

Pâmakpunga Jêsuse.

2. Nâlegar Kennuvagit NâpKoaringa, kissivit Ajornimnit

Piulijungnaramga ;

Nâlegar

Kennuêrsârvigilaunga,

Ajornimnit inûlinga,

Nekkorsitilungalo.

\section{Mel. 112.}

Er wird es thun. 645 .

1. Tussarpâtit,

Gûdib nellâgôrtub,

Ôktortaukoluadlangilâtillo,

Atâtauvor kappiasuktunut;

Mannigoininga okititsivoк, Okaupâtit illanâkardlune: Kollarnagle.

2. Ômatinga

Angmarpok illingnut

Oкагрок: Nâpkigijæksarivagit,

Kittorngara pituarigamga Aggamnit sapkotailiniarpagit ;

Kiksarnagle ikkajortauvotit,

Okperuvit.
*3. Jêsusîttauk

Igjaræksauvlune

Nuname âniatsainadlalauкрок,

Ananauvingmut paungarkârane

Piloriklune piloriktitsijok. Akkilertautsainarniarivok Sorsutsiartoк.

\section{Mel. 54.}

Das Leiden dieser kurzen Zeit, *888.

1. Siorniorner nuname Orautigijæksaungilar Ânanauvingmut, okpertut Nerriugidlartangænnut Serrivingme.

2. Taimaimat Piulijivut Âniajok mallikpavut Kunnunata sumullônêt Kappiasuktitaugupta Nâleklugo.

3. Kappiasungnek nuname Orilune sivikipor, Nâksaungitok erkarlugo, Jêsus illagigaptigo Sorairata.

\section{2\%. Mel. 14.}

Du, der Du mir in Freud' und Leid. 651.

1. Kiksarangama Jêsuse, Kuviasukuma, Pituarivagit mâne Pairitsainaramga. 
2. Attorama nellianik, Tamât kaujivarma; Idluarsijutiksamnik Issumagivarma.
3. Ôktortauvingmêtillunga Sapputilaungminga: Sangutitaulerkonnanga Pairitsainarminga.

OKPERNERUB KUVIASÛTEKARNINGANIK GÛDEMIGLO TETTEKARNERMIK.

528. Mel. $151 \mathrm{~g}$.

Ist Gott für mich, so trete. 656.

1. Gûde illagigupko,

Kia inungnille

Sâlagijungnarpângâ?

Kennuvigigupko

Kingorpiosârtipait

Akkerartortikka;

Gûb illanârikpanga

Sapputijauvunga.

2. Kristus tættigivara

Tungavigivlugo,

Aungat epкêjarpânga

Inôtigigapko,

Ajoralloarpunga

Nunamêtillunga

Jêsub nâpkigingmanga

Kujagidlarpara.

3. Tokrub pitsartuninga

Tâpsoma pêjarpâ,

Aungminut ubvarpânga

Sinnaungalernimnit;

Kujagidlalerpara

Piulilaungmanga,

Allaromangilara

Pitsartutingmanga.
529. Mel. 199.

Ein' veste Burg. 657.

1. Gûde sapputsauviksauvor Sakkuksauvok ânanamik Kappiagijaptingnillo Ikkajutsainarpâtigut;

Sâtanas kassêtok KatsungaitorsôvoK; Pingitsertornermik Sakkuksalijarpok; Nuname adsekangilak!

2. Pitsartunivut sungilak, Assiosaraidlarapta Sorsugutigivâtigut Gûdib annerilauktangat: Kinauvâruville? Jesus KristusôvoK, Nâlegax Zebaot, Assekangilarlo, Sâlakatsainartotuak.

3. Êjomadlartiptingniglo Sâtanekaralloarpat, Erksiniatsængilagut. Sâlagitsængimattigut. Nunab attanningat Kassêtogalloab 
Sotsængilâtigut;

Erkartortaugame

Kingorpiasaraidlarpoк.

4. OKautsit pêrungnangilait SokKosijuitôngmatta;

Jêsuse uvaptingnêmat Tamât tunnergutiminut; Axsârtauguptalo,

Perkutiptingnigle

Unêt pigiligit!

Suksaringilaille;

Nâlegauvik pigaptigo.

\section{Mel. 30.}

Lebt ihr Christen so allhier. 659.

1. Okpertose, inôluaritse! Sorlo Jêsus inôngmat nuname.

Âniamigut

Angergalaungmat Atâtaminut.

2. Timise tunnissigik ômunga Ittigangginullo pâmagitse Pigigapsiuk,

Pigitsainaromârivâselo.

3. Okpertut sivorlitt erkarsigik,

Mallingmatsuk kuviasungnermut:

Kivgartorningit

Igsagæksakarutigissigik.
581. Mel. 167.

Von Gott will ich nicht lassen. 658.

1. Gûd allaromangilara,

Allarniangimanga:

Mâne tessiudlarpânga

Tamartailitilunga;

Aggangminik tunnijivor

Uvamnut, kaut tamâlle,

Pattangaitidlarlungalo,

Nanêmangârmalônêt.

2. Tættigijomadlarpara

Kiksarvimne tamaine, Tagva illa kiksarnera Ablatsângortisârpa : Taimaimat tarne timelo Inôseralo tamât

Tâpsomunga perkovakka, Piniarvigilunga.

\section{1 a. Mel. 130.}

Auf, ihr Christen überwindet. 660 .

1. Okpertut! ajugautitse

Jêsub Kristub aungagut,

Tungasimatsainaritse

Mâne okausinginut.

Kaumasek-torârsiuk!

Inôser pitôngimat.

2. Jêsuse ajugaujivut Mallênarsiuk tamât!

Sâtanab illangitalo Sorsukpattigullônêt: Engelit-illavut Unnunersaumarikput. 
3. Kristub aunga pigilugo Pigâtsainarmititse!

Tamanna sorungniptingne Merngortungititsivor.

Taimaglo-sossuktor

Sâlagijautsængilak.

4. Taimak sâlakalaurivut Okpertut sivorlivut, Ajugaujungnalauramik Gûb saugârsûb aungagut. Uvagut-taimaktauk Sorsuktuksaungilagût?

5. Inôsivut Kristus illagilugo Gûdeme. Ipsomanele SakKerkattaujomârpok Tagvane-kiksarnek Kuviasungnerorpok.

6. Tagvane Gûdib kivgane Saimanersoarminut Akkileromârpait illa. Nertordlerutiniglo Tâpkoa-attorput Issokatsængitomut.

\section{Mel. 230.}

Jesu, stärke Deine Kinder. 662.

Jêsusiga sôngotikit Kittorngattit, sâlakartikit Aungnut pissiarijattit; Nutâmik inôtittigut Sâtsainarkovluta illingnut
Mungulerupta sunamut;

Gûde nertortaule

Piulijaugapta

Illûnata,

Takpânele

Pilârpogut

Inôtsib niakôtânik.

\section{Mel. 189.}

O Jesu, hilf Du mir selbst. 663 .

Jêsusiga okititsainangatôk Kennuêtitsainalaungmingalo,

Kissivit nekkoKsititsungnarparma, Aungnullo pitsartutilaungminga!

Sangêtut igvit Sôngotipattit Pigârkovlugit SâlaKarlutik.

\section{Mel. 106.}

Sollt' er was sagen und nicht. 668 . 1. Gûdivullo okarajarkâ, Mallingnago okautsine? Miksekârnek sokkos ertipauk?

Kikkarajarka ômame?

Aukak illa! okausinga Tunganartotôjor tâmna.

2. Taimaimalle ikkingnut Gûde, Jêsuse Krist, torârpunga, 
Nelliunerit nelliænne. Kuvianartunelônêt, Kiksarnartunelônêllo Tungavigitsainarlagit.

\section{Mel. 205.}

Lamm und Haupt. 678.

Nâlegar! attaviksar Okpervigênarpaptigit, Illingnik tækkonata Saimanit tungagivarut, Okpertokotivîllo

Pitsartutigidlarpât:

Saimarsainît kaut tamât Sungertûtigilugo.

\section{Mel. 36.}

O Lamm, Du Ursach' aller Seligkeiten. *927.

1. Saugârsuk! idluarsinartogavit

Inôtsiarviginartongmilutit,

Aungnulle illagêktitaumajunut,

Inukotingnut.

2. Mâne nachogijaugalloartogut,

Mittautigivlutalo taivâtigut,
Igville inukotigigaptigut Nâmaksivogut.
3. Sakkervigivluta soraingilaurit!

Sorlo mânna tikkilugo pigavit;

Attît uvaptingnut nakorijaule

Nâksaungitomut.

\section{Mel. 218.}

Wer Gott vertraut, hat wohl etc. 567 .

1. Gûdemik tættekartok, tâmna

Kilangmelo nunamelo

IgloliorsimatsiarpoK;

Jêsumik sungertûtelik

Kilangmik kingormgujidlarpoк.

Taimaimat tættigênarpagit

Jêsuse Krist Nâlegara! Mannigorutigidlarpagit Siorniornimne tamaine Kanoênimne nuname.

2. Tættigitsainaromavagit Pattangaititsungnaramga Mâne pijariakartamnik Inôtsiblo aipangane. Kakкialernekartilaunga Nutangortiuk ômatiga, 'Timigalo tarnigalo Piulikik. Tussalauruktôk Kennunera, Nâlegarale! Angênarkoniarnanga. 
539 a. Mel. 8.

Die Gnade sei mit allen. 682.

1. Gûdib saimaninganut Illagijaulaukta!

Kuviagijanganut Arvertarkovluta.

2. Аркоте amitome Pijungnangilagut, Illagijaungikupta Gûb saimaninganut.

3. Pivalliakôrpallo Siorniornivut,

Niplerpogut: „Nâlegak, „Nâpkigilauktigut!“
4. Sâlagijaukonnata, Gûb saimaninganut Illagijautsainarta Kennuêtitsijomut.

5. Allarlavut tamaita Jêsuse pivlugo, Saimanek angnerpâmik Perkutigilugo.

6. Jêsuse, merngortornak!

Niplitsainarupta: „Saimanernut, Nâlegax, „Illagijaulaukta!“

\section{OMATIB UIMAJANGININGANIK.}

\section{Mel. 208.}

Meine Seel' ist stille. 684 .

1. Tarniga Gûdeme

Pairijomajimne

Innilangavok:

Ômatima Gûdib

Aulatsivangningit

Nâmagidlarpait;

Torâtuinaruma

Kilangmut, Jêsus allarnago,

Sattômadlarpunga.

2. Attausênak tagva

Okarpunga illa,

Nagligivara:

Jêsus, nellagôrtor
Kuviasûtiga

Perkutigalo,

Tâmnaujuksauvor illa,

Perkutauvigidlartara

Inôvigilugo.

\section{Mel. 79.}

Mein Herz, gib dich zufrieden. 686 .

1. Tarnîk mannigolerit,

Issumajârungnairit

Erksisuêrlutit:

Nerriutsainaruvit,

Tagvale kiksarnitit

Okumainarungnailerput.

2. Jêsub pairivâtigut Kappianadlartunut 
Tikkitaumagupta;

Okumailuarpatta, Pêjarpait uvaptingnit, Sangênivut kaujimavâ.

3. Âkigomavlutale Kemmâtidlarpâtigut Asserornartunit, Illusekulluvullo Attorungnaikovlugit Okumainartoksôngmatta.

\section{0x Mel. 151 i.}

Den Glauben mir verleihe. 690 .

Jêsuse okpertinga

Pairijomagamga;

Pêjakit tammarnikka
Pitsainartakkale, Kakkialersârtinga, Taimaitilungalo Nâmagijarnut mâne Inôlerkovlunga.

\section{Mel. 14.}

Ei wie so selig schläfest Du. 691 。

1. Tarnîk sattortitauvotit!

Piulijaugavit

Jêsub kennermattit, illa Merngoêrserpotit.

2. Jêsusib âniangita

Piulilaukpâtit,

Okausingat kingordliub Ullapirsarmattit.

\section{OKPERTUNNIK ILLUNANÊTUNNIK, ILLAGÊKTUN-}

\section{NIGLO ANGILUARTOMIK.}

\section{2x Mel. 520.}

Gott woll'n wir loben. 692.

1. Gûd nertorlavut!

Nâpkinersoarmut

Âkisulaungmattigut,

Illagêktiluta;

Tamapta ingminut

Amulaungmattigut,

Zionib kakkangane,

Ernikut Kristusekut

Kiksamut sâlagijaunata

Perorlutale sakierkovluta.
Serrinak angijok!

Nægliktigêkaptataur

Piulijauvluta.

2. Atâtaujunit

Tussaumaniptitut,

Gûb sapputênarmagit

Pine nægligilugit;

Taimak uvagut

Tautudlarivogut,

Illagêktok̄tingine

Sakkermat ovane,

Gûdib sapputênarmattigut, 


\section{Okpertunnik illûnânêtunnik etc. $\quad 227$}

Taimaimijor nâksaungitomut;

Pinersak, Nâlegar

Gûd, nangminer Kristuse Uvaptingnêdlarmat.

\section{3, Mel. 69.}

0 wie sehr lieblich. 693 .

1. Kưianadlarmêk Nâlekab iglungit, Illagêktut

Kattimaviksangit, Mâne nertortaukovlugo.

2. Okpertokotine Innigijartorpait Maunga pijut, Uvlâmit unnungmut, Ikkinik inôguterartut.

3. Gûmik nellâgut Opigosudlarput, Pititaujut

Illagêktitarnut, Nalegauvît pigilerpât.

4. Piloridlarput

Kappianartokut

Ingergajut,

Illatik aklujut, Aungmik ajokertormagit.

5. Najudlarangne, Kiksajarangatta;

Piuligangne, Tungavigivâtit, Kuviasutsainarlutik.
574. Mel. 58.

Ein grosses Gnaden und. 700.

1. Pairksinît okpertokotingnik

Kujalijutigidlarpavut, Tâpkoa illangit Annerigangne

Pitsartutilugit aikogangne Nellojunut.

2. Illagêt serngnigiluakit, Pijomajarnut akiksokit, Nertornautiksarnut Illingatikit, Nellopkotijut piloriktikit, Jêsusiga.

545. Mel. 161.

O ihr auserwählten Seelen. 701.

1. Tarnit annerimajose! Jêsusib pairingmasse, Nekkorsijaulerkovluse, Saimanartoksoarmik Pillingmasse;

Okaritse

Oma piniarninginik, Ajornise pêjarmagit.

2. Angijor Jêsub attinga Kujagitsainarsiuk, Opigomut nertorlugo Tuksiarvigissiuk, Ôma illa

Nâpkininga

Pitjutituarivavut, Opigivlugo Gûdivut. 
546. Mel. 26.

Ihr Seelen sinkt, ja sinket hin. 703.

1. Nâlegapsingnut sâtitse

Pâmakterluse unganermut Nellopkotimut opigomut, Aungminut pissiaringmasse.

2.Uvagulle kinauvitâ?

Komuneonemik pigapta, Pijæksautinnata ôminga; Angijomik nertorlavut.

3. Nâlegarle! angerpogut, Piniarkattigilerlutit, Saimaromagangne inuit, Nangminer sagvartittigut.

4. Attausiotênartigut Attautsemut tungatitigut Ikkingnillo immertitigut Taimak illagêktitigut.

5. Najuênalaungmitigut Nâpkigijituarigaptigit, Kennuêlutit tokKogavit Pivluta nagligosumut.

\section{4\%. Mel. 161.}

Gib uns Deines Geistes Regung. 708.

1. Nâlegan Gûd, Annernerpit Kajungersaininganik Illangiutittiguttôk Ônarsitilutalo, Ungatsainarkovlutale Jêsusib naglingninganut, Tâmna najutsainarlavut.
2. Tuksiarnivut Jêsuse! Tussakit saimarluta; Saimarsainît piganerle, Okpinginek pêrlugo, Uvaptingnik ikkajorit, Mâne arvertartilluta Okpernek sôngotilugo.

\section{Mel. 114.}

Komm Liebe, komm. 710.

Nagliktotit uvaptingnêlaurittôk!

Illagêktitarnut illûnainut Saimaudsit ko vilauruk nutâmik,

Nâpkiningnernut nekkoKsilaungmigit,

Illagêllo kujagidlarlitit Pititsomagangne saimanermik.

549. Mel. 155.

Herzenslamm, Immanuel. 711.

1. Saugârsuk Immanuel!

Ômat saimanelijartor

Naglingniktok,

Tarnipta næglikpâtit;

Illalo

Kajungimut, saimanitit

Nellâgut pijomavlugit

Illuvut angmarparut.

2. Anernivut kiblikit Ônartilugit nutâmik, 
Inôsernut;

Tækkuksaulerkovlugo

Tamaine:

Illuvut innigigangne.

Tarnivullo pairksinernut, Ânanausitinangne.

\section{5็0. Mel. 37.}

Mein Heiland, bist Du nicht. 713.

Pairksijotôgavit

Piulijivut!

Kennuvogut sângne

Inukotitit,

Pairitsainalaukit,

Pijomârtome,

Sorlo naglingnermut

Pairilaurangne.

2. Mânna tikkilugo

Pairigaptigut,

Kujagivaptigit,

Piniarnitit

Pitjutigællugit;

Piulijivut

Tessiornit kissiat

Tættigivavut.

\section{Mel. 167.}

Grosser Heiland! Deine kleine. 714.

1. Jêsuse Piulijivut!

Pissiarilauktatit

Illagêt nellopкotijut

Serrivut pigigamitit ;

Mittautigijaugangamik

Tungavigênarpâtit,
Ikkingnut pairijaugamik,

NekkoKsitigidlarpait.

2. Jêsus nâpkigosungnernut

Unnuktut amuvattit

Tausendillo âlaukpattit

Saukavit iglungænnut;

Attausiolermattalo

Kuviagidlarpattit

Tessiorlugillo, Gûmut

Inôlutik nuname.

\section{Mel. 221.}

Herr Jesu, Dein freundliches. 716.

Jêsuse, kênarnut illanârnartomut

Kaumaksakit illagêktut,

Inungillo tamaita ingmikôrlugit

Naglimut nekkorsitikit;

Kivgatit tunnitsivigilaukit Ikkivit aungat pitsartutânik,

Ajokertuijunik nertornartunik

Kingômaklerkonnagille illagêktut.

\section{5อ๊3. Mel. 26.}

Was singt man doch vom Gotteslamm. *987.

1. Kanor Gûdib saugârsunga

Imgervigijungnarpittigô?

Inungnit amulaungmattigut,

Aungminut pissiarivluta. 
2. Angijunut, mikkijunut Okpertunut nakorijaule! Pairksitsiarninga pivlugo Pilloriktênarmattigut.

3. Naglingniktor uvaptingnik

Sorlo kilangme nunametauk;

Illitarigaptigo tâmna, Sumik erksijuksauvitâ?

4. Gûb, illagêktokotine Aungminut âxilaungmiligit!

Inungnut tækkuksaukovlugit

Idluarsititauningit.

\section{Mel. 126.}

Das Lamm noch eh' es hatte. *994.

1. Nâlekab uvaptitut Timekakârane Issumagidlalaukpait Okpertokotine; Mânnakullo ajulertut Kattersorpait tamaita, Idluarsardlugit.

2. Illale angijomik Mannigortauvogut, Kattangnivut Gûdemit Erkarungnairangne; Illa, Gûdib saugârsunga, Akkitudlartomulle Piulijaugapta.
3. Tamapta kennunivut Illingnut Jêsuse, Imaiput: Uvaptingnut: Nutautitsainarle Kikkiæktaunit pivluta, Tarnivullo pairikit, Aunârnît pivlugo.

4. Ikkivit aunârningit Inôtigilavut, Okpertut illûnatik Tarningit saimakit, Kuvianartok kilangme, Toraræksarilugo Nerriugidlarpât.

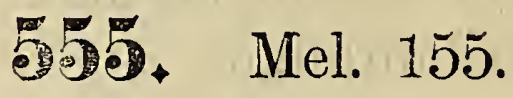

Unsere Seele harrt auf Ihn. 738.

1. Tættigivavut Gûde Sullinata pingitogut, Inôtsemut, Sapputênarmattigut, Tarnivut

Nekkoksititsainarmagit, Uvaptingnik naglidlarmat, Kujagitsainarpavut.

2. Illænnâkartor illa! Naglimut okausekartok, Saimarsaijor,

Piuluatangitut Kaikovait, Illagêlle pairilugit, Illanâkarninganiglo Tamapta okssivogut. 
3. Amen kiblingnartornik Illuvut illumertauvut; Sorairane

Ôma inukotinne
Tamaita

Nêklkoksititsainarmagit;

Napkigijæksaunginivut

Missigitsainarpavut.

\section{ILLAGÊKTU'T TUKSIARUTINGINIK.}

\section{Mel. 185.}

Gnad' und Heil' und Friede. 749.

1. Saimanermik ullapirsaumiglo

Pexarlit Jêsumêtut,

Nâmagijaujomamut ômunga

Ingminik tunnidlartut:

Illa inôvigitsainarsiuk

Kivgartuênarsiuk ômamut,

SoкKоsijuidluse

Ungagissiuk mâne.

2. Illagêt! mallitsainarsigiktôK

Piulijipse Jêsub

Okausingit, pivluta sêktaujub

Ômatimigut aglât;

Opigomut piniarisiuk

Pitsartutipse pijomajanga;

Kuviagisiugle

Pivluse inôninga.

3. Tâpsomane peratsainaritse .

Tussugidlartapsingnik,

Tamainik pitsainaromadlarmat
Okpertokotiminut.

Kiksarangapse mannigorlise,

Arvertarnipsingne saimaningat

Illagitsainarlise,

Nekkoksitiluselo.

\section{5\%. Mel. 102.}

Preisswürdig's Lamm, ich ehre Dich. 756 .

1. Nâlegak! opigivagit, Ittiggangnullo pâmakpunga,

Tâpkoa kuviasumut Kugviornermut kunnikpakka:

Unêlle tarnimne piunginama

Nellunginivagille pigigamga.

2. Pairrsijotôgavit illa, Saugakotingnutânaptigut, Illingnit tammarkonnata Attiptigut taisârpaptigut; Nellâgôrutsillo nutângortitsuk

Ikpinartilugo ômatiptingnut. 
558. Mel. 83.

Erdlingnarnerpângojor Ungagissiuk tamapse, Saimarsailune Jêsub Mannigudlaromavâse; Uma nagligingmasse Mânna Kuviasugitse.

\section{Mel. 159.}

Ajortuggit naglingmagit Jêsub kaikoluse, Mânna aggane issakpait, Erkitsomavluse, Tâpsomunga aisâritse Saimaninga attorsiuk; Ullapirsautiminiglo Tunitsivigingmasse.

\section{Mel. 155.}

O Gemeine, freue dich. 750 .

Illagêt serrititse!

Kujagisiuk tamapse

Nâlegase,

Saimanartovaksuit

Pivlugit,

Attortase tâpsomangat, Inôsipsingnelo tamât Tukkisijungnartasse.

\section{Mel. 151 a.}

Du gestern und auch heute. 753 .

Ikpeksar uvlomelo Nâlegax Jêsuse!
Kittorngavit illûnatik Missigivâtille, Kaut tamât najornivit. Ullapirsauvillo Illummertimattikik Illatit tamaita.

\section{Mel. 56.}

O Du theurer, werter Bräutigam. 754 .

1. Nâlegarle :,: najudlartigut!

Kennuvogut :,: saimarsaigavit,

Ômativullo aungnut Tamaita eprêjakit, Sorairata :,: nagligilutit.

2. Ajornivut :,: pêjalaukittôk,

Ikkingnullo :,: auliksoarnut

Ijertortigut mânna, Tagva inôtilluta

Nâletsaina :,: romavaptigit.

563. Mel. 79.

Gesinde des Getreuen. *1033.

Illagêt Nâlegangat, Engelit serrigijangat, Pingortitimiktut; Ajortuggit illipse Tokrotigilaungmasse, Kanok Kujagivisiûk? 
2. Inôgutigijavut

Nelliumat, tokkomut

Pivluta pijotit,

Nâpkiginadlarapta

Taitsiarnaugitomik

Piulijaukovlutale.

3. Tarnipta naglikpâtit

Anernipta ikkitit

Najoromadlarpait,

Unêt suksaungitogut

Tokkotigigaptigut

Akkiksârnut pigitigut.

4. Saimartigut Jêsuse! Attorpogullo tagva

Nangmagæksaptingnik;

Idluarijarnulle

Inônasuarpogut

Nâlegomadlaraptigit.

5. Jêsuse! tussartigut, Nellojungnaititigut Atternik, tamapta Ijertudlartitigut Ikkivit illuænnut,

Pivluta tokkolauravit.

\section{Mel. 166.}

Wir wollen alle wie wir sein. 762 .

Illuêngarluta Gûmut

Tunnijomadlarpogut

Uvaptingnik, inôvluta

Ômunga kissianut;

Kaujivogut Jesusivut,

Pivluta tokkogavit,

Taimaimat âriksortigut

Tokkovit kaitanginut.
565. Mel. 208.

Unserm Gott und Lamme. *1059.

1. Saugârsuk Gûdivut, Tokкojok rejungme.

Piulivluta,

Ânianartomut

Tokkomullo aijok

Pissiariluta,

Jêsuse nertortaule

Tâmna ômamut tamânut

Orautigilavut.

2. Illagêt kugvise

Kajungerniselo

Kaujimadlarpait,

Agviarkattartut

Omasiptingnêtut

Pêjaromavait,

Missigitiluselo

Piuliklernerminigle

Soraijuitomik.

3. Jêsub ikkinginut

Nippitsainaritse

Ajorsarapse,

Inôtigivlugit

Kaut tamầt nutâmik

Kannimajotut;

Taimak tamapta pita!

Piulijiptingnut illa,

Angergarkârata;

4. Tækkokârnagolo

Jêsus ikkingillo:

Itteruptalo

Nerrimarvinganut,

Pijarêrtanganut

Illûunaptingnut: 
Tagva erkilârpavut,

Kunniklugolo ômamut, Piulingmattigut.

\section{Mel. 184 a.}

Nâlegavut nakorivavut Illagêt pissiaringmagit, Pulivlugit kattitimmagit Inônialerkovlugit Ôma idluarijanganut, Issuanit kennermagit, Taimaimalle nagligosumut

Najorpait pairilugillo.

\section{5ீ6\%. Mel. 121.}

Im Friede Jesu Christ. 748. 766.

1. Mânna nagliktavut,

Illavut taunane

Jêsusib Kristusib
Ullapirsautâne

Salutidlarpavut

Jêsub tamapse

Tækkonarlise :,:

2. Uvagut tamapta

Kattimalerapta

Jêsuse! tokkungnut

Onartilauktigut

Missuktiluta aungnut;

Kattangutivut

Illûnânêtut

Illagilugit.

3. Jêsuse tokkungnut

Najulaungmittigut!

Nunakattivullo

Europamêtullo

Amerikamêtullo,

Asiamiut

Afrikamiut

Kattangutivut.

\section{UVLOKSIUTINNIK.}

568. Mel. $22 \mathrm{f}$.

Herr Jesu Christ, Dich zu uns wend'. 767 .

1. Najortigut, Jêsusiga! Anernit tillilauruktôk, Ôma tessiorlittigut Аркоtiksame illingnut.

2. Kannivut angmartilugit Ômativut nâlektikit, Illitarigannerlutit OkperKovluta illingnut.
3. Pakkoa illagilugit

Takpâne nertoruptigit

Taututsainalârpaptigit

Kuviagitsainarlutit.

\section{Mel. 205.}

Geht, erhöht die Majestät. 768.

1. Illagêt Nâlegangat Ânanaujox nertorsiuk! NâlengneK nâmagivâ Nertutsiarangaptigo. 
Inukotingitalo

Ômamut nâlengningat

Nertornautigiclarpâ,

Toknotigigamigit.

2. Jêsuse Nâlegase Malliklugo nagliksiuk, Pingmase saimarluse, Kujagitsainadlarsiuk! Saimaner tussarnertor Tessiortigivavut Kilangmut, saimanermik Kaujijok pilorikpor.

3. Illagêt kemangnagit Najutsainalaukilletôk, Piuliklertetôgavit Tættituarivaptigit. Tamaita allarpavut Ikkajortetôgavit, Tamât pingortitavit Nâlegaksarivâtit.

578. Mel. 56.

Jesu Kreuze. 770.

1. Jêsusiga :,: âniagavit, Naglikpagit :,: ungagivlutit,

Tokkokasaleruma

Tagva ômalerpunga Âniarnik:,: tussaleruma.

2. Nâlegavut :: ikkajortigut, PerKojarnik :,: malliktittigut
Tarnipta aut kissiat

Piulijiksarivât,

Okpernivut:,: angijororle.

\section{Mel. 26.}

Mein Heiland, wirf' doch einen Blick. 771.

1. Piulijivut! mânnakut

Takpângat tækkonârtiguttôk,

Kibliktigut, illa tamapta

Kaumanernut saimartigut.

2. Saimanernêtsomav ogut, Pijomavit tessiorlittigut, Kaumaksatsainarlittigullo.

Tammarkonnata illingnit.

5\%+. Mel, 146.

Ach Schöpfer meiner Seel'. 777.

1. Pingortilauktiga, Aungnut piulijiga, Kiksajarangama Kuviasuktitjga ;

Pivît niakoat Kivgarnut Nâlegar Illagêt angutât Nâlengnartoksoak;

2: Nâlegatuara Gûde okperigapkit; Tokkut auliksoak

Pituaridlarpara; 


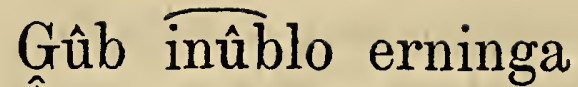
Ânanaunît illa Allartailivara Angergarkârnanga.

3. Âniavigijarnut Aungmik kidjijârvingnut, (Kênarnit nunamut Kuttit Korlulaungmatta,) Tagvunga illusikka, Issumakullukka Illûnaita illa Illijartorpakka.

4. Uviniga illa

Pingmalle uviningnit, Taimaktauk saunikka Saunernit pingmingmatta, Taimaimat timiga, Tarnigalo mânna, Sujungaititsainakik Nunamêtillunga.

\section{5\%3. Mel. 79.}

Gib mir ein lichtes Wesen. 778.

1. Kaumanekartilaunga Inûlidlarmingalo Illupkut tamapkut; Ikpigitillungalo Asserorsimanimnik, Saputinga ajortomit.

2. Kuviasugviginga Atâtak ! pigingmanga Ernerpit aungminut;
Anernek! pairilaunga ; Illa Piulijiga!

Illûnarma pigilaunga.

\section{5\%4. Mel. 208.}

Was wir immer müssen. ${ }^{*} 1085$.

1. Soraijuitomik

Missigijæksaujor

Omatiptingne

Imaipok: Gûdivut

Ânialik toккотак

Missigivlugo,

Timivut tarnivullo

Sujungaititaukovlugit

Jêsusib aunganut.

2. Taimak kissiane

Nellâgut ômame

Mannigorpogut,

Jêsusib aungminut

Inûlingmattigut

Ajorniptingnit;

Tuksiaruptigolo

Kaut tamât inôsiptingne

Pairidlarpâtigut.

575. Mel. 141.

1. Kattimalerapta

Illagittigut,

Ikkitit pivlugit

Saimausertigut

Erkaititigullo

Piuligaptigut, 
Uvlullo tamaita

Najulertigut.

2. Illagêlerapta

Piniarnernut,

Okartuksauvluta

Nagligusernik,

Missigitittigut

Ulıpkosênernik, Aungnut ômativut Missuktênakit.

5\%6. Mel. 184 a.

Hier sinkt, o Lamm, zu Deinen Füssen. 784.

1. Sêrroortorvigivâtit mâne Jêsus illagếKotivit,

Pissiarigangne Kejungme,

Ômamut kujagivâtit, Aumavlutiglo kugviormut, Napkinît saimarsainîllo Illume missigigamikik Ônarsitigivlugiglo.

2. Okautsit Jêsub ikkinginik, Aungmik kiægungninganik,

Nellojungnaitidlarpâtigut Annerijaumaniptingnik. Illagêt sungertûtigivait Sâtaniblo erksigivait; Uvapuingnut itteromakpat Jêsub aungat agviarpâ.

3. Kuviasutigivât tagva Illagêt, tungagijatik,
Idluarsijutigivâllo Aglissivalliavlutik; Gû̉b Annerningat tessiorpait,

Aungat illagêktimmagit, Saimarsautingat nâlektipait

Tamainik sâlakarlutik.

\section{5\%8. Mel. 9.}

Unser Gottes Lämmelein. *1091.

1. Gûdipta Saugârsunga Akkonaptingnêpor Sennerangat aunârtub Tarnivut sakkerpait.

2. Okautaujungnangimat Missigijæksauvok Tautulerangaptigo Ikkit kaumaningat.

598. Mel. 30.

Aeltster Deiner seligen Gemeine. 789.

1. Illagêt pairijingat, saukavit

Nakoridlarlugo saimarsainît,

Kujagivâtit,

Aungnut, tokkungnut piuligangne.

2. Illagêktitavit kennuvâtit Attannetuarijomagamitit 
Ikkingnut aungnut Axiksolaukit nertornautingnut.

\section{Mel. 70.}

Die Wahl der Gnaden. 786.

1. Saimanernulle

Kennilauraptigut

Nertorpaptigit

Unêt ajortogut,

Nâpkigosuktotôgaville, Sangêtut pitsartutivakpattit.

2. Illagêktose!

Jêsub saimarlise,

Kênangatalo

Kollangiarlise!

Anernipsigut ârlortose.

Tâpsomunga ikkinginullo.

3. Ikkinginulle

Perkojomavogut

Illûnaptingnik

Issokangitomut.

Ikkimine tunnilittigut

Piloringnermik nâksaungitomik.

\section{Mel. 195.}

Gûd uvaptingnêpor

Ullapirsartivut

Kuviasûtigivavut;

Naglingniktoksôjub
Inôjub nuname,

Najorpâtigut tamâne:

Kiksartut Keajut

Kugvingit allarpait,

Erkitsomadlarpait.

\section{Mel. 230.}

Souverainer Herzens-König. 790.

1. Nâlegaviksôjotille

Tækkonâlaukit nâlektitit

Illingnut angersimajut;

Illagêt, Kaikolauktatit

Mâne sângne pâmangarlutik,

Kanoxtôdlalerlutiglo

Saimanartovingnut

Nâmagomavlutik,

Sakkerlugo,

Serrinivut, Jêsus igvit

Mâne najorangaptigut.

2. Kennuvigivaptigille,

Ittervigitsainalauktigut

Sângne kattimagaigupta;

Okâlanerlo tokkungnik

Piniarnerilauktarniglo

Pitsartutitsainalauruk.

Tuksiarpaptigit

Kaujitilauktigut

Perkojarnik

Ajornartut uvaptingnut

Ajornarungnaitilaukit.

3. Illagêt kattersorangne, Igvit pairijigingmattillo 
Akkorngænnêtsainalaurit; Ahâk! Illagêt Jêsuse Najorungne serrivut, illa! Mannigortigingmattille; Pikit pairijivut, Kennunivut igvit Tammartulle, Sâtisâkit, nâpkigikit, Tamaitalo uttertikit.

\section{2x Mel. 166.}

Willkommen unter Deiner Schar. 791.

1. Akkonaptingnêlerittôk! Kuviagigaptigit,

IllagêktoKotitillo

Najornernut saimakit;

ToKkungnigle orautikit

Naipertugaringmattit, Têssiutsainalaungmigit Attanneringmattille.

2. Anerniptigut aggaktit Puttoggit kunnikpavut Issaktitaujut pivluta Senningajolingmulle. Attortatit kongmuarlutit Illatit saimarlugit, Nangervigilârtangattauk Okpertut, tikkivingne.

3. Illagêt, akkonapsingne Jêsumut inôngitut, Ômunga pijomangitut Sulle, kapsiolerкât?
Kailauritse illûnase Kikkitaujokarane, Kuviasûtiksapsingnik Tunnitsivigingmasse.

\section{Mel. 166.}

Verliebter in die sel'ge Schar. *1103.

1. Pivluta inôlauravit, Pivluta tokkogavit, Uvaptingnulle kissivit Nertoræksatôvotit. Ahâgle Piulijivut! Ârlorpogut illingnut, Ullapirsautingnik mânna Tunnitsivigittigut.

2. Mânnale Piulijivut Tuksiaromavavut, Tâmnalo ômatiptingne Missigitsainarlugo; Ômattivullo tamaita Aungminut saimarlugit Miksexârtome ovane Itsainarkovlutale.

3. Tuksiarvigiguptigo, Jêsub tussarpâtigut; Sujungaitidlarlutalo Aungminut; assianik Kajungilârungnairapta Mâne inôtilluta, Kissime Nâlegar illa NagliktôK uvaptingnik. 
4. Jêsusele sângne mânna Tagva kattimavogut, Kujagivlutit tamapta, Piulilauraptigut, Akkonnaptingnêlaurittôk! Saimartilaungmitigut, Timivullo tarnivullo Aungnut serparsarlugit.

\section{Mel. 159.}

Der Sabbat ist um's Menschen will'n. 796 .

Inuk pivlugo Sabbate Illingakojauvor, Nekkoksijaukovlutale Jêsub ômatâne,

Tâmna tokKungmat pivluta,

Attorpavut makkivia, Merngoêrviksaukovlugo Tarniptingnut illa.

\section{Mel. 159.}

Geschöpfe zur Geburt gebracht. *1106.

Illagêngortitaujose

Kristuse tokkungmat Aulingmillo sennerânit Erniangovose.

Tarningat idluarsarlise, AnerneKattigiluse, Ikkiliksôjub timingat Pairiniarlise.
586. Mel. 26.

Ein jeder Tag ist gnadenvoll. *1108.

1. Nâpkigijauvigivavut

Uvlut tamaita, Nâlegapta Pingmattigut, angerpavullo,

Nâleklugo naglingnermut.

2. Nuname illagêngnivut Soraerkonnago, nâleklugo, Attinganik kujaliluta, Nellâgut kittorngaujotut.

3. Nâlegaujor illagênut Pitôgavillo, taimaimalle Tættigivaptigit, ômavit Illagitsainarmattigut.

\section{8\%. Mel. 185̆.}

Herr und Aeltster Deiner Kreuzgemeine. 798 .

1. Nâlegar nâletsainarpâtille Illagêt nagliktavit, Kutsaservigijomainarpâtit Pairijigingmattille;

Opigivlutit ungagivâtit, Kugviorput tussumut illingnik,

Niakorigamitit

Pituarênarpâtit.

2. Jêsus, kênavit sângane mânna

Tagva kattimavogut, 
Kujagiviklutillo tamapta Pissiarigaptigut

Âniarnut aungnut ikkingnullo;

Pigitigut tamanna pivlugo Idluarijarnullo

ÂkiksulauktiguttôK.

3. Mattomanelo uvlut tamaita

Kattimajarangapta, Âniarnik, aungnik, tokKungniglo

Erkaumatsainarluta, Anernekullo ajunginekut Ômativut najuênalaukit, Ajokertortimingnik Nâletsainarkovlugit.

4. Taimagle ittikkatit puttoggit

Salutitsainarparut,

Illûnata opigosungnermut Nachoginginaptigut,

Illagêkotingnut ânaptigut Inôniarkovluta illingnut;

Mattomanêtilluta

Kuviasukovluta.

5. Atâtavut serngnigênartigut,

Pairiniarlutalo;

Gûd Anernerlo nâpkigitigut,

Tessiutsainarluta,

Jêsub ikkinginullo aunganut

Anertaudlartomut timinganut;
Piulijivut aungnut Serpakit ômativut.

588. Mel. 184.

Nâlegak! kattersoigavit Inungnik illannâkamut, Aniarnut pissiarigangne, Ittiggangnut pâmakpogut, Ómamut kujagivaptigit;

Kujalineк, Gûde, piuk! Tarnib mannigorvigingmattit,

Nertordlernek nâmagiuk.

\section{Mel. 114.}

1. Gûd pairikit tamât kattititatit

InôgutiksaKartilugillo

Tammartut kenneriartulaukit,

Kaikolugillo uttertilaungmigit;

Kannimajut inûlilaungmigit,

Illûnaita issumagilugit.

2. Jêsusiga, illagêt Nâlegangat

Attît uvaptingne nertornarpor;

Kingurâvut piloriktikittauk,

Illingnullo kittorngautilaukit

Saimanernut illangiutitikit 
Sorlo uvaptingnut pilauravit.

\section{Mel. 166.}

Herr Jesi, sei uns selber nah'. 780.

1. Nâlegak Jêsus nangminek!

Najortigut ovane, Ajornivullo tamaita Mânna pêjalaukittôK, Kejungme nivingalutit Pivluta tokkogavit, Aggangnut puttomajunut Naglimut saimartigut.

2. Jêsuse najudlartigut Perkutigigaptigut Angijomik kiksarpogut, Tammarnivut pivlugit, Illûnâne ajorapta Tuksiadlarpaptigit: Ikkajortaujomavluta, Idluarnersaukovluta.

591. Mel. 121.

Nâlegar Jêsuse, Illûnata mânna Tuksiarpaptigit, Najulaungmittigut, Aungnullo ômativut Tamaita ubvakit, Pairilugillo tikkiviksarnut.

\section{Mel. 151.}

Jêsuse! mânna sângne SerKortolerpogut
Tuksiarvigivlutit:

Ômatekulluvut Illumertilaukittôk Ullapirsautingnik ; Ungagapta illingnut Najutsainartigut.

\section{Mel. 9.}

Jesus Christus blick' dich an. 782.

1. Jêsub tækkonârlise Illagêt illipse,

Aungmut pissiaringmase Perkutigivâse.

2. Naglingniktotôgame, Illannarivâse:

Nunamêtillusele Tâpsomanêtitse.

3. Ikkinginêleritse Mallitsainarlugo Ômamut kennutitse. Opigidlarlugo.

4. Ôma naglingningane Agliserkovluse, Idluarijanganut Inôtsainaritse.

\section{Mel. 30.}

Hebe auf die durchgegrab'nen Hände. 789 .

1. Aggaktit puttomajut issakkit

Illagêktitarnut, saimalugit 
Saimarsautingnut

Ômavit kajungilârningatut.

2. Illingnut tarnivut perkovavut,

Perkojatit malligomavavut

Kuviasumut,

Pairksinernut nekkorsitittigut.
595. Mel. 164.

Ach mache dir doch jegliches. 799.

KanoKtôK nertornautingnut

Illûnata nuname

Illingatitsainartigut!

Takpaungartuksatitut

Itsainarkovluta,

Anernivut illa

Tarnivullo timivullo

Pitlaræksaulerкonnagit.

\section{BAPTIJUMIK HAILIGEMIK.}

\section{Mel. 132 a.}

1. Illagêt opigugitse

Mânna erkaumagapta Jêsub saimarsaininganik Illagêktitaminut, Ômatiminik naglimut Tættætomik uvaptingnik, Kaujitilaungmattigut.

2. Nâlegak taimak pivlugo

Kujagênarpaptigit, Illagêktilauraptigut Epkêjarluta aungnut: Kennuvogut naglingnernut Illumertilaungmittigut, Ungagapta illingnut.

\section{Mel. 161.}

1. Piulijiptingnut tamapse Kaikojauvose maunga
Illagêlerkovlusele,

Nâgliktigêkluselo; Inuillo illûnatik

Tussaumalerkovlugille

Tokкominut piulingmase.

2. Okpingitut akkorngænne Arvertarkâlaukpose, Mânnale baptijutikut Pêjarpait ajornise: Jêsusetor nagliksiuk Tâpsoma naglerkârmase; Ikkinginut nippititse!

\section{Mel. 56.}

1. Illagêktut :;: Kuviasugitse Piulijipse :,: kaikolaungmase,

Illagêktitaminut

InôKovluse Gûmut, Mattomane :,: inôtilluse. 
2. Taimak illa :,: Piulijise Ômamullo :,: nagligissiuk Tâpsomalo ikkingit, Issumagênarlugit, Tokkunganut :,: baptitaugapse.

599. Mel. 14.

1. Nâlegar Jêsus Kristuse ! Aut kovilaurangne Inôgutekarkovlugit, Tamaita inuit.

2. Manna tuksiarpaptigit : Tâpkoa inuit Aungnut sujungaitilaukit Baptitautillugit.

\section{Mel. 23.}

1. Jêsuse! kattimavogut Tuksiarluta illingnut, Ômativut Anernernut Aungnullo najulaungmigit.

2. Immer tâmna tækkovavut, Aggullo missigivavut; Sennerarnit puailajub Ubvarligit ômativut.

601. Mel. 206.

Jêsusiga :,: tâpkoale, (una mânna)

Baptitaujut, (joк)
Torkokit, (ruk) :;: ikkingnut,

Pissiarigangne :,: aungnut Tokrungnullo,

Illivavut ômatingnut

Kennuvluta, tessiutsainakit, (ruk) :,:

\section{Mel. 22.}

Gott sei gelobet und gepreist. *1112.

1. Gûde nertutsainarlavut

Timivullo anernivut

Issumagiluarmagit

Piloriktitsomavlugit.

2. Kristusib piniarningit, Kejungmêdlune tokKungmat

Puailajotun-idlarput Âkiktaujutigivavut.

3. Tâpkoa baptijutikut Illangiubvigivavut, Okpernerme aulangitor, Nellâgut piloriktipat.

4. Taimaimat inôtilluta Tættigidlarpavut tâmna, Tagva ajortaptingnigle Pisârpâtigut tamainik.

5. Kanoktôk Gûb okausingit Tussaumajaulit tamaine, Pissiarijangit tamaita Baptitaurovlugit aunganut. 


\section{Baptijumik hailigemik.}

\section{Mel. 84.}

Liebster Jesu wir sind hier. 804.

1. Jêsuse mânêpogut

OKautsitit nâleklugit:

Unale nutarârsuk

Piuk, imâk okaravit:

Nutakкat uvamnut pilit,

Kilaub pingit pigingmagit.

2. Taimaimat kennuvogut:

Tâmnatauk pairilugolo

Illangiutititsuk

Pingnik, nâpkigilugolo,

Kittorngariuk nuname,

Kingormgujititsuk pâne.

3. Jêsus! aungnut ubvaruk

Idluitunit tamainit,
Ômûna ubvautekut

Ulliguk pitsartunernut;

Ajorningit pêjalaukit

Attitilugo illingnik.

4. Pairksigavit, saugârsuk

Piuk, pairiniarlugo ;

Unertuijok, kittorngat

Kilangmut tessiorlugo:

Akkerkotut napârtomut

Taimak illingnêtilauruk!

5. Jêsuse naglingnernut

Tussalaukit Kennunivut

Nâmaksitilaukillo,

Tâmna tuksiugaptigo.

Attingalo aglalauruk

Aglangnut ômarnartunut.

\section{KOMMUNIÔNEMIK HAILIGEMIK.}

605. Mel. 182.

O Lamm! Du branntest vor

Verlangen. 810.

1. Kingorlermik nerrimârnernik

Jêsus rillanâlaukpotit;

Tarnivit tagva ungagivait

IlliniartoKotitit;

Kannernit ômanadlartor Inôtsib kônga kôktidlar-

$$
\text { pat, }
$$

Naglingnerub aumatipâtit Illingnik raujitinangne.
2. Naglingningnît innornadlarpok

Taimak tattamnartullijox, Pitit nerritinangne timingnik,

Aungniglo immertinangne. Suglugiviuk Jêsuse!

Kejungme tokKotigiluta? Illuptingnêtsomavotillo Tarnipta nagligijingat.

3. Naglingningnît pitsartudlarpok!

Tokkomit sôngonersauvOK ; 
Naglingnermut tunnergutauvor

Pingortitivut nangminek; IIluvermut pitipâ, nunab Kilaublo sajûtigijangæek, Tamaita inôtigijangat Taimagle naglingner pivor.

\section{Mel. 159.}

Wie ofte hat mein Herz geweint. 824.

1. Illupkut keavakpunga

Sannikterivlunga,

Issumagama Jêsuse

KiksarpoK pivlunga.

Ômunga pairijaulunga

Sulle inôlunginama

Ôma kuviagijanganut

Kangusudlarpunga.

2. Jêsus Nâlegatôjotit

Illagêktitarnut,

NekkoKsititsainarpattit

AkkeKangitomik

Ingminek nellopkotijut:

'Tagva ajoksadlarama

Illumnelo kiksarama

Nâpkigilaungminga.

\section{Mel. 22 a.}

Was weinst $d u$, warum girrt die Kehl'. *1147.

1. Illumne sôg keadlarkîk? Tammarnikka erkarapkit;
Jêsusiblo tarninganut

Kugviorpunga ungamut.

2. Mattomane illagêktut, Sulle innersimangitut Ajulitsainaramigle Korlorput Kugviorningit.

3. Angergarkojaukârnanga Jêsumut, nunamêdlunga Timinga aungêrotijor Najutsainaromavara.

4. Kilaublo ânanauninga Tækkogupkolônêt mânna, Jêsusib aunâdlarninga Ânanaugiluarpara.

5. Pissiarigamga aungnut, Jêsuse tuksiarpagit: Timingnut âniajomut, Inûlinga ajornimnit.

\section{0\%. Mel. 14.}

Es segne uns Gott, unser Gott. 783.

Gûd, Gûdivut saimartigut Sakramentekotingnut, Nerrititigut timingnik, Immitigut aungnik.

\section{Mel. 9.}

Bis die Stunde kommt heran. 813.

1. Nelliuxârtinnago Jêsub sakkervia, 
Tækkuksaukârtinnago

Inukotiminut.

2. Erkaumanartotuamik Pillilaukpâtigut,

Pivluta tokKominik Erkaitipâtigut.

3. Timiminik aungminik Komuniôneme, Aitortormattigulle, Kujagidlarlavut.

4. Nekkoksitipâtigut Timingat aungallo, Inôniarkovluta Illa Gûdiptingnut.

5. Tagva Gûdib Erninga! Umamut tamânut Nakoridlarpaptigit, Inûligaptigut.

6. Akkunaptingne, unêt Tækkuksaulerlutit Arvertaromatinnæk Missigivaptigit.

7. Tagva time tarnelo Sângnut pâmakterput; Timît nerrigivlugo, Immerlugo aggut.

8. Nekkorsijaurovluta Timivit aukpillo Pitsartutipâtigut, Najudlaraptigit.
9. IllagêktoKotitit

Komuniôneme

Pitsartutilaungmigit

Timingnut aungnullo.

\section{Mel. 97.}

Der wahre Mensch und Gott. *1130.

1. Pititaugame unnuak Jêsub tiguvâ каккôjak, Avgorpâ Apostelinut, Kujalivlune окагрок: Tâmna nerrisiuk inôtsemut

Pivluse timiga tokkotauјок.

2. Ermgusiarsuk Wainelik Kujalimut tigugivâ, Tâpkonungalo tunnivâ, Imâglo okarvigivait: Tamapse tagvanget immeritse,

Tagva auga kovijok pivluse.

\section{Mel. 99.}

O Handlung voller Majestät. 815. Piniarnek ussornartok! Naglińgneк nelleкangitor! Nelliutor hailigeojor! Tarnivut nekkorsilerput: Tоккunga окаutigivarut, KomuniồneKarangapta. 


\section{Mel. 22 a.}

O Jesu, lass uns allezeit. $* 1133$.

1. Jêsus Komuniônemut

Killanârtitsainartigut,

Ômamut kajungertomut

Timît aullo attorlugik.

2. Kannimasivut âkikit Sujungnivut pêjalaukit; Igvit nâpkoarivluta Aut kovigangne pivluta.

\section{Mel. 14.}

Je näher man dem Heiland. ${ }^{* 1134 .}$

1. Pigannerpalliagupta

Piulijiptingnut,

Ajornek, idluarnerlo

Kaujiluarpavut.

2. Tagvale kêmitikpogut

Âriktaujomamut, Inôniaromagapta

Kuviagijanganut.

3. Nellekangitub tâpsoma Naglikamittigut, Tuksiarnivut tussâvait, Piksarserkovluta.

4. Timiminik aungminiglo, Komuniôneme, Inukotine aitorpait Nekkorsitilugit.
613. Mel. 83.

Er nimmt mehr sich uns'rer an. 819.

1. Saukanik pairksijomit Gûb pairiluarpâtigut, Ômatinga angmarpor Nekkoksititsomavluta. Saugakutaumavogut Aungmut piulijaumajut.

2. Kâktut immeruktullo Mattominga illûnatik, Nekkorsivut ômunga Orautaujungnangitomik: Tâmna adsigivlugo Pâne perkârtinnata.

\section{Mel. 36.}

Was könnte wol beim Gläuben. 820 .

1. TækkoKârnago okpervigivlugo,

Komuniôneme nerrigaptigo

Timinga, aungalo immeraptigo,

Uvaptingnêpor.

2. Tagva Jêsub Kannimajorotine

Inûlidlarpait nellekangitomik;

Naglimut kiblingmagit ômativut

Ômartitauvut. 


\section{Komuniônemik hailigemik.}

3. Ômativullo kajungilâdlarput

Adsigijomadlarlugo Gûdivut;

Timivut tarnivullo tâpsomunga

Killanâdlarput.

\section{Mel. 149.}

Gûb Erninga nagliktor Tækkonâlavuttôk, Inûliklertotuak Ajorniptingnille: Ômunga aingmita! Inûlingmattigut Illâle aungminut.

616. Mel. 166.

Mein Heiland, der Du uns zu gut. *1139.

1. Piulijiga! pivluta

Kikkiaktortaujotit;

Ittertotillo kilangmut

Aungnulle nangminernut:

Mânna illagêktitatit

KomuniôneKârtut,

Nerrinermut immernermut,

Illingnut kivitikit.

2. Nellâgut ômanartomik

Okpernek sakkijârle, Okperluta Gûudiptingnut; OKautigidlarlugo
Inungnut tamainut, illa Inôtilluta mâne;

Nagliktigêngniptingnelo Pivalliakovluta.

\section{1\%. Mel. 23.}

Schmücke dich, o liebe Seele. 822.

1. Tarnîk opallungailaurit

ErKarlugolo nagliktit, Kaumajomullo ailerit Ôma piksarserkongmattit.

2. Jêsub najoromavâtit, Timiminik aitorlutit, Tâmna kilangmêgalloartor

Mânna illungnêtsomavor.

3. Pagreartorvigilugo

Umatingnut erkileruk,

Nachoginiangilâtit, Illanâridlaramitit.

4. Ômatit angmaruk mânna Imâglo okarvigiuk: Tarnimne ajoksarama Ikkingnut kaivunga mânna.

5. Illingnut Piulijiga!

Ômatipkut kâdlarpunga, Kaitarnullo mattomunga . Kugvinut killanârpunga.

6. Timipkut sajulerpunga Illupkut opigukpunga; Nellekangimat pijara Kujagidlarpagit mânna. 
7. Jêsuse каккôjaujotit, Illa kilangmit pijotit, Ikkajunga sullinanga Pingnik nerrilerkonnanga.

8. Mâne ittiggangnêpunga Illangiutitjlaunga Kilaub pinganik ôminga Piulijaujutiksamnut.

\section{Mel. 242.}

So oft wir in Erwartung stehen. 823.

Nerriugviptingne tamaine Jêsub Komuniôninganik, Ômativut illungertudlarput

Kiksamut serrimullo; Serrimut, Jêsusib piminik Pillingmagit;

Kiksamut, inugoininga Ôma, inuKotigijomavluta Kammagitsianginaptigo, Nertutsiarnagolo. Ômat ajortolik Okpertorle tussuror: Kanortôk aggakpit puttortaujut

Nâpkigilittigut, Nekkoksilutalo Ullapirsautingnut.

\section{Mel. 141.}

Sünderfreund voll Gnade. 825.

1. Saimarsaijôgavit,

Tuksiarpagit,

Ajutsainarama,
Nâpkiningnernik

Missigênartinga,

Najornerniglo;

Kinaunera igvit

Kaujimagangne

2. Nâpkiningnît Jêsus

Missigivara:

Ajoksarneralo

Ikpigivara,

Pitlaræksaunimnik

Okautigupkit

Igvit okauparma :

Piulivagit.

\section{Mel. 23.}

Naht heran, ihr lieben Glieder. 828.

1. Illagêt najûtititse, Unêt ajoksadlartose, Jêsub aunga immerlugo Timingalo nerrilugo.

2. Mânna Jêsub timinganut Tarnivut illa kâdlarput Taimaktauk immerudlarput

Ômartitsijub aunganut.

3. Illagêt, opigilavut!

Jêsusib najormattigut, Nekkorsititsomavluta Timiminut aungminullo.

\section{Mel. 22 a.}

0 dass nun Jesu Kreuzgemein'. 829.

1. Jêsub illagêkotingit

Uvininganik pigamik, 
Aunganiglo immeramik, Tokkotaulit ajorningit.

2. Tarnivullo timivullo Idluatsainarkovlugit, Inôsiptingne tamaine Ungalerta timinganut.

3. Gûb kuviagingitangit Illuptingnêtut tokxolit! IkliguneK ajortomut, Sangusarainerlo tamât.

4. Illa Jêsuse tokkungnut, Nutâmik âkigaptigut; Tamât idluidlarnivut Tokrotikit âniarnut.

\section{Mel. 36.}

Sein Schweiss im Busskampf. *1152.

Jêsusib kidsijârninga, pivluta

Aunga senningajolingmêdlune kôktor,

Timingalo tokкomut tunInôtigivavut. nijaungmat,

\section{Mel. 145.}

Wenn ich ihn essen kann.

1. Timinga nerrigapko, Tagva inûlivânga, Aunga immerapko,
Nekkorsivor illuga;

Perkojanga tậmna

Attorpara mânna

Ajornikka illa

Pêjarpait tâpsoma.

2. IllagêKotitit

Aungnut piulijattit,

Aitortulaungmigit

Timingniglo aungniglo

Mânna najortigut,

Kingorngagulletauk

Pigannerkovluta

Illingnut tamapta.

\section{6\%4. Mel. 69.}

1. Piulijivut

Illagêktitavit

Ovanêtut

Tuksiadlarpâtit:

Timingnut nekkoksidlartigut!

2. Jêsus, timingnik Nerrijomavogut, Najortigut!

Ômatiptingnele, Killanârapta illingnut.

\section{Mel. 22 a.}

Gemeine, zitt're heiliglich. 829 .

1. Illagêt sajuleritse!

Piulijipta ômatise

Mânna kiblingniarligit!

Tokzome pitsartutânut. 
2. Illagêt kuviasugitse Piulijipta tarnise Timiselo timiminut Ivsornaitiligit mânna!

3. Illagêt, kuviasugitse! Timinganik nerrigapse, Tokkunganik erкaikovluse

Imailiorkolaukpâse.

\section{Mel. 185.}

Drück' uns die Empfindung

Deiner Schmerzen. 833.

1. Ômatiptingnut nênertolaukit,

Anianitit tamaita,

Ittikkatit pivluta puttoggit

Omamut kunniklavut;

Kugviornermut kennuvaptigit,

Timingnut ikkilersimajomut

Pairitsainadlartigut,

Missuktiluta aungnut.

2. Ômativut ônarsivut tagva Jêsuse âniarnut, Aungnut tokkungnullo illuptingne

Nekkorsitigaptigut;

Tuksiadlarpaptigit ômamut,

Tarnivut aungne inôtsainarlit:
Tokkut nakkorivlugo

Angergarkârtinnata.

3. Nekkorsinartor Komuniôneme

Okautaujungnangilak, Ômatipta upkoangagulle Ittervigikpattigut, Tokkomelo pitsartuninganik

Ômativut illumertippagit; Ullapirsautinganik Tagva missigivogut.

\section{2\%. Mel. 184.}

Sein Fleisch- und Blut-Genuss gibt Leben. *Anh. 217.

Jêsusib timinga aungalo. Illangiubvigilugik. Inôsezartitsivok illa, Ajortoêrtilutalo.

Saimanarmêk! Sakramenteme

Kibliktaugupta Jêsusib Tokkungat pitsartuninganut,

Ittervigikpattigullo.

\section{Mel. 71.}

Auf Seele! schicke dich. 834.

1. Opallungailerit Tarnîk! Piulijît

Pâmagvigiuk, 
Nâlegaujotojub,

Kakкôjakut Wainekut

Ingminik pivâtit.

2. NâglingneK angnerpâK!

Kiblingnartorsoar!

Nâpkigingmattit

Nellekangitomik;

Aglâttauk Engelit

Ipaksingilât.

3. Uvanga kinauvîk?

Igvit Nâlegavik

Itteramgale,

Timingniglo aungnik

Komuniôneme

Aitortoramga.

4. Pijomajarnulle

Igvit каiкogamga

Kaisâdlarpunga,

Jêsus okaravit

Aitoromavlunga

Tættigivagit.

5. Tagva okpernermut

Ômamut illingnut

Kellaksortinga!

Komuniônemut,

Timipkut tarnipkut

Inûlilaunga.

6. Tokrotaujok timît,

Inuit pivlugit

Tunnilaurangne;

Nerkigilerlugo,

Pitsartuninganut Akilaungminga.
7. Aukpit saimanartub

Tattamnartitsijub

Kiblidlarlinga,

Iptekadlartomik;

Inûliklertomik

Immertilaunga.

8. Akkerkotut illa

Iptekartiminga

Taimak illingnit,

Perortilaungminga,

Inôniarlunga

Idluartomik.

629. Mel. 32.

Ich fall' in Staub vor Dir. 835.

1. Sângnut Jêsuse pâmakterpunga,

Sanningnit mingnerpângodlarama ;

Anernermale nertorpâ saimanît,

Opigomut, kunnikpait ittikkatit.

2. Ômatiga pannertotun-ipor, Nellungilatillingnut rêlertor,

Sangêtunga, pigigamga nellâgut

Ukperlunga katjârpunga illingnut.

3. Perkutaulangaletôk illingnut,

Komuniôneme aitoramga, Timingnik aungniglo; opigosumut 
Erkilagit, nekkoksitigigapkit.

4. Suksaungigalloartunga sângne,

Taimaimalle naglingnît sakkerpok

Ajoksartor nekkorsitidlarlugo

Tagga ômattiga, nekkoKsititsuk.

5. Angidlarmat oKautaujungnangilak

Tunnergutit, kinauvigle sângne?

Nâlegaujok, uvangalo illikut

Attausînangortitaulermigamnuk.

6. Issumairotivungale sorlo Nâlekama ittervigingmanga,

Aiparijomavlunga inôsimne:

KujanaK nellâgut pilorikpunga!

7. Jêsus attorpâkka timît aullo

NekkoKsitigiluarlugiglo;

Illingnullo inôjomadlarpunga,

Illûnamnik illingnut tunnilunga.

8. Sângne okausiksakarungnaipunga,

Okarille igvit, nagliktara!
Sorainanga, illingnêtsainarlanga,

'Гоккullotauk erkaumatsainarlago.

\section{Mel. 97.}

Der Othem, der die Todten regt. 836 .

1. Anernek! tokкungajunik Tamât ômartitsijotit, KiblikittôK illagêktut Imanuêlib pijangit: Ermgusiarsuk immersimavoK

Jêsub aunganik ômanartomik.

2. Gûmut illagêngojose, Aumalerlit ômatise! Waineliksablo napârtub Iptingallo, pitsartôjub Jêsusib aungat kiblidlarlise

Kovimajub piulidlarmase.

\section{Mel. 70.}

O Blut der Wunden. *1161.

Jêsub aunganik

Ilkpigilerapta,

Ômativulle

Opigosudlarput;

Nâlegar! ussornadlaravit, Aggulle inôtigidlarpavut. 
632. Mel. 23.

$O$ dass bei den Wundenbache. *1162.

1. Ikkinginit kôktomulle Tarnivut ômartitaulit, Kuviasuktitaukovluta Unganermut tâpsomunga.

2. Piniarnivut okautsivut Nertordlerutiksaukovlugit Ômamut opigilavut Kuviagijaukovluta.

\section{Mel. 11 a.}

Jesu Wunden thun sich weit. *1164. 1. Jêsub ikkingit angmarput Aunga nipliagivok: Immeruktor Kailerle! Jêsus, immerukpunga.

2. Piulijiga aulik!

Imitsuk sennerarnit Ajoksartok tarniga, Saimartitsuglo mânna.

\section{Mel. 184 a.}

O heilig's Blut des Lebensfürsten. 837 .

Jêsusib aunga ikkingillo Uvaptingnik inôlêjut, Kêmigangapta tâpsomunga
Pagreasârivâtigut;

Inûlinartut ômanartut Nekkorsitipait tarnivut, Ikkingita puailavingat Pitsartutidlarpâtigut.

\section{6อือ. Mel. 79.}

Lamm Gottes, Deine Wunden. 1167.

1. Jêsusele; ikkitit

Sulle uvlut tamaita

Saimanartoksôvut,

Tâpkonungalo sâtut,

Kuviasumut okarput:

Piksaritsainaramigit.

2. Aungnut piulijatit

Uvlome erkilaukit

IllænâKarnernut;

Ikkingnelo torkokit,

Aungnullo missuktikit

Ômattingit nekkoksikit.

\section{Mel. 70.}

Vergönn' uns stündlich. *1168.

1. Uvlut tamaita

Ikkingnêtitigut!

Tâpkonungalo

Ungatsainarapta;

Nerrinermullo immernermut

Illûnata pitsartutitigut.

2. Illagêngnivut Aulajuititsuk 
Nagliktigêngneк

Tækkuksaurovlugo.

Uvaptingne, nâpkigosumut

Aggangnut tessiutsainartigut.

\section{3\%. Mel. 149.}

$\mathrm{O}$ die Seligkeit ist gross. 839.

1. Piloringner angivor

Taitsiarnangilak:

Jêsuse okpernikut

Pigimariklugo ;

Sullele ungamut

Nutakkatut sorlo

Keagênarlugo.

2. Inûliklertub aunga,

Killanârivara

Kônganit Kalluvakka

Kuttit, immiksakka;

Ômunga tarniga

Ajoksarkattartok

Kêniatsainarpok.

\section{Mel. 26.}

Halt' uns Dein Herz stets aufgethan. $*_{1073 .}$

Ômatit angmatsainarle. Kippivigidlaraptigille Ômativut nekkoKsitikit. Aungniglo immertitigut.

\section{9+ Mel. 69.}

Dein Blut wir trinken.

Aut immerparut

Mânna kajungimut,

Kivitikit

Tarnivut ikkingnut

Tagvane nekkorserovluta.

\section{Mel. 58.}

Gott ward im Fleisch für uns offenbar. *1170.

1. Gûb nelliuninga nelliumat Uvinekarlune sakkerpok, Ullapkoserluta

Âniaminut,

Piulivluta ajorniptingnit Tokkominut.

2. Jêsub timinga Gûb perKovâ,

Tarniptingnut ovanêluta

Nerkiksaukovlugo,

Taimaglo aunga,

Ungagijaptingnnt aikârata

Immiksauvok.

3. Okperaptale okarpogut

Inungnut Jêsub tokKunganik,

Taimaglo aunganik;

Kovijaumangmat, Tagva Gûde ullapirsartauvor

Pivlutale. 


\section{Komuniônemik hailigemik.}

4. Uvlut tamaita. imailerta: N̂Alegar kujagidlarlugo, Ânanaudlarninga, Agganginelo

Ittiggangine ikkingit augTautukôrlavut.

\section{Mel. 185.}

Gloria des Lammes Wundenhöhlen. 840 .

Nakorijaulit Jêsub ikkingit Illûnaptingnut mânna, Tamapta ajoksatsainarapta Killanaridlarlavut.

Timiminik nerritippattigut, Aungminiglo immertidlarluta,

Timivullo tarnivut

Tagva nekkoksijauvut.

\section{2, Mel. 22 a.}

Gott seiłgelobet und gepreist. $* 1173$.

Gûde nakoridlarlavut

Timiminiglo aungminik Mânna aitortormattigut, Inûlivlugit tarnivut.

\section{Mel. 132.}

1. Piulijima Jêsusib

Aitortutsainarpânga

Timiminik aungminiglo
Tâpर̄oa inûlivânga; Ikkiminut ajornikka Pêjarlugille tamaita, Mannigutsainarpânga.

2. Ômat auktojor pivlunga! Sumik akkilerlagit? AkkilêneK ajorapko Illûnarma piginga! Kidsijârnivit pivlunga Sennerkavillo ikkingat Kibliliuk tarniga.

3. Kugvinut kujagivagit, Tokkungnut piuligamga, Kennerivarma inungnit, Sulle ajulaurama, Aungnut pissiarigamga Kittorngarijomavlunga Nuname, kilangmelo.

\section{Mel. 151 a.}

$\mathrm{Du}$ uns so nahes Wesen. 841.

1. Jêsusivut najornît Missigênarlavut! Illumertinaptigut, Kujagivaptigit! Pijominarpok mânna, Testamente una, Illûnaptingnut illa Nertortaukovlugo.

2. Ômâtit, issumallo Jêsub ikkinginut Itteromadlarpulle. 
Innigênarlugit;

Pituarilavulle

Kikkiaktortaujor,

Timingat aungêrotub

Inûlingmattigut.

\section{Mel. 146.}

Herr Christ, ich danke Dir. 842.

1. Nâlegar Kristuse!

Tagva illa ômamut

Kujagivaptigit

Nagligilauraptigut;

Toknotigiluta

Tunnidlaraptigut

Timingnik aungniglo,

Nekkorsekovluta.

2. Nagliktor Jêsuse

Puigungilaptigit

Timingnik nerrigapta,

Aungniglo immerapta:

Mânna ômativut

Illingnêtsainarlit,

Illuvut illingnik

Illumertilugit.

\section{Mel. 107.}

Mein Jesu, der Du vor dem Scheiden. 843.

1. Jêsusigale, toKkoKârnak, Kingordlek taimna unnuak,

Siornioravit âniarnut, Pitsartutitaukovluta
Komuniôn illilaurangne

Uvaptingnut, nertorpaptigit.

2. Tamanna attorangaptigo, Erkaumatsainarpaptigit Missigitidlarpaptigullo Ônartomik naglingnernik, Ânianitit aullo tokKut Ômatiptingne nutângorput.

3. Timît inôgutigivavut Aut nekkorsitigivavut, Illuptigut ômarikpogut Pingnik tunnivakkaptigut: Kajungilârpogut ômamut Nâletsainaromadlarlutit.

4. Timivit avatinginulle Attausiotitaurogut, Tâpkoa uvagullo sângne Tarnekattigêlerpogut Timingnut aungnullo tamapta

Nekkorsijaukattigêkapta.

5. Timîllo pitjutigivavut Timiptatauk sangêdlartut. Sanningmit makkitsomârningænnut Ânanausimariklutik, Nungusuitomiglo pâne InôseKaromârniptingnut.

6. Jêsus! ivlernartoksôjunik Piksakartidlarpaptigut, 


\section{Komuniônemik hailigemik.}

Komuniônemele igvit

Nangmineridlaraptigit,

Unêt sulle âniavluta

Piloreutigivavut mânna.

\section{4\%. Mel. 82.}

Weil ich Jesu Schäflein bin. 844.

1. Gûb kittorngaringmanga Kuviasudlarpunga

Nagliktima annerijima

Pairivlunga naglikpânga,

Illitarivlungalo,

Attimnik tailungalo.

2. Nâlekab iglungane Illagêksimajune,

Tâpsoma nerritipânga

Timiminik, kâkonnanga,

Immerulerangama

Aungminik immipânga.

3. Kuviasudlarpunga

Piloridlarlungalo,

Uvlut mânele nâkpatta Angergaudjaularamale, Pairijimnut kilangmut Nertornartotuamut.

\section{Mel. 23.}

1. Jêsuse mânna toKkokit

Idluartomut timingnut

Illuptingne idluitut, Ajortuggit avativut.
2. Ônadlartomullo aungnut Ômativut ônartikit, Ikkingnullotauk aulingnut Tessiutsainadlartigut.

3. Inungnut tamainut tokkut Okautigijomavavut, Naglikluta uvaptingnut, Nagligidlalauraptigut.

\section{Mel. 102.}

Wenn ich im heil'gen Abendmal. *Anh. 228.

1. Tokкut erkaumadlarlugo, Komuniôn attorangapko, Erкailungalo Nâlegar Kanok pissiarilauramga: Tagvale, Jêsuse, inûlilaunga,

Inôsertâmiglo pitilaungminga.

2. Ânianitit nênikit

Taimak ittijomik tarnimnut

Pijitsertornît uvamnik PuigortaujuerKovlugo; Tagvale, Kaut tamât, serrigivara

Ittertuksaunera nâlegauvingnut.

\section{Mel, 22 a.}

Wir danken Dir, Herr Jesu Christ. $* 1182$.

1. Jêsus! kujagivaptigit Pivluta tokrolauravit, 
Ajornivullo tamaita

Timingnut illilaurangne.

2. Komuniônît hailige, Uvaptingnêtsainalerle; Timiptalo tarniptalo Inôtetuaringmattit.

\section{Mel. 23.}

O Du Herz voll Gnad' und Liebe. 847.

1. Ômatille tættædlarmat Naglingnermik uvaptingnik,

Mattomunga aumavogut Ômame kiblikaptigut.

2. ToKkonît illûnaptingnik Kiblêdlarmat, ômativut Nippinganasuadlarput, Serkomitomut timingnut.

3. Nekkoksidlalauraptigut, Inônasuadlarpogut Gûb idluarijanganut Illa ômamut tamânut.

\section{Mel. 22 a.}

Die Frucht vom heil'gen Abendmal. 848.

1. Tunnergut Komuniồneme Ivlernarnerpângojorle Pigigaptigo, uvagut Nagliktigếnojauvogut.
2. Pinele kemmakârnagit Tuksiarutigigamigit Nagliktigêngnex Jêsusib Tâpkonanêtsainarkovâ.

3. Tamanna Testamentinga Nâmaksekojominarle! Illagêt nagliktigêmut Kellaksormatta ômamut.

4. Ômat, aggait pitiparut, Sokkosijuêromamut, Nellagut inôtilluta Nagliktigêgomagapta.

5. Tikkitsomârninga taimak Neriugilugo kaut tamat, Opallungaitsainarluta, Illeragijomanago.

6. Okpernermik illûnata Atsuilitsainarluta! Nâlegar ikkilertaujor TækkoKârnago pânetor.

\section{5อ̉. Mel. 23.}

Wenn wir arme Sünder könnten. 850 .

1. Ikpigijaptingnik mânna

Okarluta ajorpogut;

Ônarmatta ômativut

Jêsusib najorninganut.

2. Engelit illagivlugit Piulijipta sânganut 


\section{Komuniônemik hailigemik}

Sêrkortorluta ômamut Gûdivut nakorivavut.

3. Illûnatik oKautsivut Amigârput, ussornarmat; Ômativut pikit mânna Tunnitiksakanginapta.

4. Mânna kujagivaptigit, Pakkoa illagivlugit. Timît nerrilauraptigo Aullo immilauraptigo.

\section{Mel. 121.}

Der Vater segn' und hüt. 852.

1. Atâta! pairikit

Inungit Ernerpit!

Jêsuse, kênarnut

Kaumartilaungmigit!

Anernek ajunginek

Tækkonâlaukit,

Uvlut tamaita

Kuviasuktikit!

2. Tussarnidlartunik

Nertordlerutinik

Attausênaujotut

Imgerkattigêkta!

Illanârijivulle
Opigosumut

Nakorilavut!

Najormattigut.

655. Mel. 151. Aria.

Da sind wir arm und blöde.

Kikkiætortaugavit

Uvanga pivlunga, Tagva nakorivagit llla Jêsusiga.

KanoK pinniarlunga Akkilêjomavlunga?

Pigidlalaungminga Illûnarma illa.

\section{Mel. 185.}

Eine andre Stunde lässt $\mathrm{Du}$. schlagen. 798 .

Kennertaujut baptitaukovattit,

Idluarsitilugit

Ajungitomut tessiorpattit, Okarvigivattillo:

Komuniônemut pitigapse, Timimnik aumnigio tunnivapse!

Mannigoromavluse, Illumnêtsainaritse! 


\section{INGMIKÔRTARTUT IMGERUTINGINIK.}

a) INGMIKORTARTUNUT ILLUNAINUT.

\section{5\%. Mel. 37.}

Heil'ge Dreieinigkeit. 853.

Gûdivut, tamainik

Pingortitsijok

Ullapirsaijorlo,

Idluarsaijok

Tuksiarpaptigit,

Piloriktikit

Illagêktitaujut

Ingmikôrtartut.

\section{Mel. 58.}

O Du, dess Güte kein Mund ausspricht. 856.

1. Naglingnît ipaksinangimat:

InuKotitit tækkonâkit,

Uvlullo tamaita

Kattititatit,

Ingmikôrtartut najutsainakit

Saimanernut.

2. Aungnut serparsalauktiguttôk

Piniarniptingne tamaine!

Missigerкovluta

Najornernigle,

Sorlo sâptingne nangeraville

Tækkuksaunak.
3. Naglimut pingortipaptigut, Aungnut pissiarivaptigut, Pilorerkovluta

Nâksaungitomut:

Anernerpillo illagêktunut Âpâtigut.

4. ÔKautigijæksarivavut Illiornitit ajungitut Inungnut tamainut; Anerniptingnut, Timiptingnullo nertorlaptigit

Sorairata.

5. Jêsuse tuksiarpaptigit: Illingnut adsiutittigut, Tukkiserkovlugit Inukattivut, Innigingmagit Piulijipta Ômativut.

659. Mel. 23.

Dass der Sinn des Lamm's Sinn gleiche. $* 1200$.

1. Jêsusiga! issumaktut Taimailaulit issumavut: Time tarnelo âKikik, Ikkilertamut timingnut.

2. Illitarimarikluta, Saugakotingnilk tailuta, 
(a) Ingmikôrtartunut illûnainut.

Tokkungnut âniarnullo, NekkoKsititsainartigut.

\section{Mel. 215.}

O grosses Evangelium. 63.

Okautsit tussarnernerpât: Gûd uviningme sakкerpoк, Inuit perkutimetut Aungminut piulilugit! Inukattigêt illûnatik Mânna attutsiarligit

Piniarnerijangit,

Kiksanarvingmut kaigame Ajornivut errsuklugit. Tokkotigilutalo.
661. Mel. 70.

Mein einzig Flehen. 857.

Piulijiga,

Kennuvigivagit!

Ingmikôrtartut

Tamaita saimakit,

Illingnullo tessiulaukit

Tamaine kuviasukovlugit.

\section{Mel. 79.}

Nun, erstgeborner Bruder.

Kattangutigijavut Angervigivaptigit:

Nâlegomavlutit

Inôtsivut nâlugo,

Nellagôtsainarluta

Ungagijomagaptigit.

(b) SORRUTSIT IMGERUTINGINIK.

6ี่อง. Mel. 79.

Appersût. 1. Nagliktakka, tamâne

Sôgle kattimavisê?

Kiolersingale!

Kigusек. Tussaromadlarapta

Pilorilerkovluta, Kattimalerpogut mâne.

A p. 2. Kia pingortipâse, Angminullo tarnise Piulilaukpagit?
Kig. Gûdib Piulijipta Pingortilauklutalo Piulilaurivâtigut.

A p. 3. Tagva piulingmasse Illagêktimasselo Nagligingilassê?

Kig. Aukak, nagligivarut Pissiaringmattigut. Aungminut ikkiminullo.

A p. 4. Jêsub sorrusiolune Sôgle angajokângne Nâletsainarpagîk? 
Kig. IgsagæksaKarkovluta Tappatokonnatalo

Nâlektitsomavâtigut.

A p. 5. Inumariolermat

Kanox illiolauka

Nunamêtillugo?

Kig. Atâtab rilangmêtub

Perkojangit mallikpait,.

Sennavorlo aggangminut.

A p. 6. Merngortorpalaungilar

Kâlaungilarlônêllo

Kiksalaungilarlo?

Kig. Aukak kiksadlarlune

Tamainik ôksilaukpor, Aungmik kiægudlarlune.

A p. 7. Tamaita ajornivut Pitingmagit ingminut

KanoK illitipât?

Kig. Avatingit puttorpait Sennerâlo kappivât,

Tagva immeк auglo kồkpuk.

A p. 8. Suksauvâk tamakkoa
Korlortuk immeKauglo

Jêsub senerânit?

Kig. Pêjarpait ajornivut, Idluarijanganut, Inôniarkovlutale.

\section{Mel. 20.}

Jesulein, man kann es lesen. 861.

1. Jêsuse, tussalaukpogut, Nutarârsôlaungmigavit, Piulijekarkovluta, Asserorsimagapta.

2. Aglaksimavor illingnik, Sorrutsit nagligigangne, Kaikolugillo illingnut Nuname-itillutit.

3. Taimaktauk naglikaptigut, Ômativut eprêjakit, Unnuktullo tammarnivut Issumagijungnaikit.

4. Taimaktauk angajokârut Tuksiuparut illingnut, Pivlutit pairingmattigut Saimartidlarniakit.

\section{6อ. Mel. 149.}

Was hat denn ein Kindelein. $* 1211$. 1. Kanor tagva sorrutsit Jêsus adsigivât? 


\section{(b) Sorrutsit imgerutinginik.}

Timâtut timekarput

Aullo tâpsotunak:

Sorrutsit okpertut

Jêsub inôsinga

Igsarasuarpât.

2. Jêsus erkaititigut,

Tappatoniptingnut,

Kuksalainiptingnullo

Kiksartigaptigit;

Taimaitut, Jêsuse

Illaginginangne,

Serngnigilauktigut.

3. Sorruserle Jêsuse, Tammalaungitotit!

Ajornekadlarapta

Tuksiarpaptigit:

Kaut tamât nuname

Inûlilauktigut

Piuliklernernut.

4. Jêsuse, naglingnernut

Ônarsitilaukit

Tamaita ômativut.

Ajornek pêrlugo

Illingnut ailuta

Tuksiarpaptigit,

Sujungaititigut.

\section{Mel. 14.}

Ich bin ein kleines Kindelein. 863 .

1. Sorrusiogama mânna,

Pitsakidlarama

Jesuse tuksiarpagit

Saimartilaungminga.
2. Piulijiga pivlunga Sorrusiolauktotit, Ajortunit attê aungnut Piulilaungminga.

3. Ômatigalo illunât Kaigapko illingnut, Piuk kaujimadlarapkit Perkutigigamga.

4. Sulle kaujerkârtinnanga

Kittorngarigamga,

Baptijutikullo illa Aungnut ubvaramga.

5. Ômatiga serngnigiuk,

Sallumainingalo

Ubvarlugo, erdligiuk,

Nagliktor Jêsuse!

6. Nunamêkojomagumga

Jârekadlarlunga,

Attortuksangortilunga

Kivgaksarnut tagva.

7.:Perkojatit malliklugit Arvertarlauruma, Uvlukka tagva nâkpatta. Illingnut pitinga.

\section{6\%. Mel. 141.}

Das ist Jesu Freude. 860.

1. Jesusib sorrutsit-tækko gunigit

Malligasuartut - okausinginik, 
Kuviagilerpait, - kujagivâllo

Piulilaungmagit - tokkominulle.

2. Nutarauniptingne - baptitaugapta,

Illa Gudiptingnut - tunnijaugapta;

Ômanêtsainarta! - ajorniptingnit

Ubvalaungmattigut, - illa aungminut.

3. Jêsus Anernerpît - pairilittigut

Ômativut aukpît - aumatiligit

Nâpkigosungnîllo - pêrniarnago

Pigingikuptigo - ajudlarpogut.

4. Ittiggangnut mânna-serKortorluta

Tuksiarpaptigit,-- saimarsainernut

Tækkonâlauktigut - kiblingnartomik,

Pissiarigaptigut - akkitujomut.

\section{Mel. 22 a.}

Drum sollen die Unmündigen. 859.

Taimaimat sorrusiarsuit

Okautigijæksarivâtit
Kejungme tokrolauktotit Tamainik pingortitsigavit.

\section{Mel. 70.}

Ach gebt euch alle. 865 .

1. Illûnaptingnik Tunnijomavogut

Jêsus illingnut Ânialauravit;

Torkortilauktigut ikkingne,

Inôtsiarkovluta sângne.

2. Ittiggangnullo

Tagva pâmakpogut

Tuksiarluta

Pikit ômativut!

Ajortunit sujungaitikit,

Nagvârtitigut saimarsainernik.

3. Ungasiktullo

Illingnit, Jêsuse,

Amualaukit,

Pijomadlarangne,

Nagligosumut kennilaúkit Gûdemut kittorngaulerKovlugit.

4. Kaikogaptigut

Kailerpogut tagva,

Ajoksartunik

Nâpkigosuktotit!

Uvlome sulle ubvartigut Aungnut idluitunit tamainit. 
(b) Sorrutsit imgerutinginik.

5. Kangusukpogut

Nippunganiptingnik.

Kennuvaptigit:

Idluarijarnut

Ârilaungmittigut nuname

Piloriktitigullo takpâne,

6. Âniajotit

Tessiulauktigut

Pititigullo

Kittorngakotingnut,

Aungnut pissiarilauktarnut.

Pijuksaujunullo najugarnut.

\section{6\%0. Mel. 79.}

Du Blutverwandte Liebe. *1222.

1. Uvineкakattivut!

Jêsuse naglingnernut

Kajungertitigut

Sangêtut pairigangne

Sorrutsit nagligangne

Kilangmut ittertitigut.

2. Aggut kovilaurangne Ômunga kibliktigut, Nutaulerkovluta Sujungaititigullo Kittorngautilutalo Gûdemut adsigilutit.

3. Asserorsimagapta Illûnata Kaivogut Pâmagvigivlutit Kennuvogut kugvinut
Ômamut killanâmut

Nutâmik nâpkigitigut.

4. Nâpkigosuktôgavit

Tuksiadlarpaptigit

Unganikinivut,

Tappatonivullotauk

Issumagijungnaikit,

Nagligitsainartigullo.

5. Illænâkarnît illa

Angimat uvaptingnut.

Erdligivaptigit,

Tarnivut timivullo

Tunnivavut illingnut,

Pituaridlaraptigit.

6. Jêsuse nagliktivut Igvit Kaujivaptigut Tokkungalaurapta Tamapta kattangnermut Sorrutsit ômatingit Illingnut ungatilaukit.

\section{Mel. 22 a.}

Though but a little child I am. 1016.

1. Sorrusígalloartunga Tоккоtaujok Jêsus illa, Nortorlago, naglingmanga Unêt ajoktorsôjunga.

2. Tættigivagit Jêsuse Sorrutsillo naglikangne, Nunamêdlutit pigavit: "Uvamnut sorrutsit pilit!" 
(b) Sorrutsit imgerutinginik.

3. Pitiminga Jêsusiga! Nelloprotinimne sângne, Ubvarminga ajortunit Aungnut, nagligomagapkit.

4. Ajortunut unganermit Jêsuse sapputilaunga; Nâleklanga perкojarnik, Pâne tækkojomagapkit.

\section{6ชึ. Mel. 159.}

How heart-affecting Christ to see. 1039 .

1. Illa kiblingnartorsôvor Kristus tækkolugo, Ânialerkârtinnago Naglimut pivluta, Jerusalemut ailune, Kaglilermat, apkotânut Kessertutit annorâtiglo Inuit illivait.

2. Imgerput Hosianamik Taimaktauk sorrutsit, Taivâllo illagêklutik Attinga serrimut; KanoKtôk sorrusiptingnut Tâmnatauk Gûb saugârsunga,

Pivluta tokkotaulaungmat, Nertortaule mânna.

\section{6\%3. Mel. 4.}

Mein einziges Gut. *1226.

1. Illûnaralo

Inôseralo
Tunnidlarpakka

Illingnut Jêsuse, piulilaunga!

2. Piulijiga-ajortôjunga Tuksiarpunga

Aungnut ubvarlunga pigilaungminga.

\section{Mel. 39.}

Ihr Kinder, wo seid ihr unfehlbar. 867 .

Appers ût; 1 . Sorrutsit kiale sapputidlarpâse, Pairidlarluselo kiale pivâse?

Kigus eк. Jêsusib kissime sapputivâtigut, Illagêktullo pairidlarpâtigulle.

A p. 2. Kattimalerapse sumik tussarpisê?

Kig. Jêsusib aunganiglo tokkunganiglo ;

Tâpsoma ikkinginik tussarangapta

Kujalivigidlarlavullo tamapta.

A p. 3. Piulijisele капок pijaulaukâ?

Kig. KikkiaktortaujoK kejungmut pivluta, Aggangillo ittiggangillo aunârput, Illa illûnane ikkilersimangmat. 


\section{(b) Sorrutsit imgerutinginik.}

Ap. 4. Taimak âniangmat kinale pivlugo?

Kig. Illunarsoata ajortoKsôgapta;

Tamanna pirlugo tokкоvor pivluta, Aungminut piulijomavluta illa.

A p. 5. Kujagivlugole piulilaungmasse. Tâpsomunga sumik tunnijomavisê?
Kig. Ômativut ômunga tunnidlarparut,

Nagligivlugolo nungusuitomut.

A p. 6. Kialo ajorungnaitinivâsele?

Kig. Piulijiptale aungminut kissime;

Tamanna pivlugo nagligidlarpavut, Inôsiptingnelo nakoridlarpavut.

\section{SAIMARTITSINERMUT.}

\section{Mel. 10.}

1. Sorrutsit perkutitit Mânna pitsartutikit, Kunniklugit tamaita; Tussartigut Jêsuse.

2. Sôrrusiolauravit Ômatingit pigikit Aungnut serparsarlugit, Pêrkonnagit illingnit.

3. Ubvarsimajut aungnut, Pigannertilaukille Unganerme illingnut, Nâletsiarkovlugit.

4. Illingnêtsainartikit, Pannilerkonnagille,
Aungnik erdlingnartomik InôguteKartikit.

\section{Mel. 185.}

O Du, aller hilfsbedürft'gen Sünder. 869 .

Ajulertunik tamainik igvit

Inûlêje Jêsuse,

Sorrutsittauk angiluartomik

Nâpkigijomagangne, Ingmikôrlugit kattilugillo, Ajortunit amualaukittôk! Gûd idluarijarnut Perorniarkovlugit. 
(c) Nukapitsat nullêtullo imgerutinginik.

(c) NUKAPITSAT NULLÊTULLO IMGERUTINGINIK.

687. Mel. 37.

$O$ dass wir Kindlein sein. 871 .

Pijominadlarpoк

NutakKatitut

Itsainarkovluta,

Inôtilluta,

Taimaigupta illa

Kilangme sorlo

Inônajarpogut

Gûb najugâne.

\section{6\%8. Mel. 22 a.}

Ihr lieben Knaben allzumal. *1233.

1. Nukapitsat illûnase,

Gûd igsagæksarijase

Nukapiak inungortor

Jêsuse tækkonârsiuk!

2. Perortor silatovlune, Saimarsaivalliagame, Sangijôvlune tarnekut, Gûb, inuillo sânginut.

3. Nukapiangoniptigut Igsagæksarilerlavut, ÔtunaK ivsornailuta Ubvautigilugo aunga.

4. Uvaptitulle- ilaungmat, Illinganivut illûnât Ôkpâa, ajorneK pinnago Akigomagamittigut.
679. Mel. 164.

Ihr lieben Knaben, wenn ihr wollt. *1237.

1. Adsigijomagaptigit Jêsus Piulijivut Inôjomadlaraptatauk Kuviagijarnulle; Ômativut tagva Kaipavut illingnut Aungnut sujungaitilaukit Idluarsitilugillo.

2. Arvertartilaungmitigut, Tamât nâmagijarnut, Okpertullo âkkorngænne Piniartilauktigut Gûb nertornautânut, Nellâgôrtitigut Okpernekartilutalo Taimailingatitiptingnik.

3. Tarnimigut tinimigut

Jêsusib tokkunganut,

Piuliklerninganullo Idluarsititaujok, Attaruinermik Nâletsiarnermik Issumalijuernermiglo IllangiutititauvoK.

\section{Mel. 214.}

Amen, ja ihr lieben Kinder. 873.

Jêsub piuliklerninga Piksar angnerpâr tâmna 


\section{(c) Nukapitsat nullêtullo imgerutinginik. 271}

Pijominarnerpângongmat, Tuksiutigivavut, Tarniptigut timiptigut Asserorsimanivut Ikpigidlaraptigo, Sâvigivaptigit, igvit, Piuliklertetôgavit Ajortunit tamainit.

\section{Mel. 58.}

So oft du aufstehest. 876 .

1. Uvlâkut tuppalerangapta Jêsuse erkaitisârtigut Kujalinermigle.

Sinniktilluta

Najorluta serngnigigaptigut

Unnuame.

2. Tuksiarvigivaptigittauk Tarnivullo ômativullo Aungnut missuktikit, Erkarsautivut,

Taimaktauk tamaita orautsivut, Serparsakit.

3. Nellojungnaitilaungmitigut

Ikpigitsainalerkovluta:

Tarnivut timivut

Uvlut tamaita

Sujungaititaugiakarmatta, Aungnut illa.

4. Issumaksarsiortitigut

Uvaptingnik appertsorluta:
Ajornekarnimnik

Ikpigilerkîk?

Ikkingitalo inûlingmanga

Missigivîk?

5. Issumagijaujungnainermik

Idluinimnille tamainit

Tuksiarkattarkîk? Piulijima

Aungminut pêjalaungmangâgillo

Kaujimavîk?

6. Arvertatsainaromagama

Passijæksaungitomik sângne,

Nâlegomavakka Gûb oKausingit.

Igvit aungnut ubvatsainarminga

Ajornimnit.

682. Mel. 166.

Was ist ein wahres JünglingsHerz. 877.

1. KanoK illingajuksauvât Inûsuktut okpertut? Tâpkoa Jêsub ikkingine Inûlijiksarsivut.

Nellâgôrtomik nertorpât Jêsub akkitojomut Aungminut kovimajomut Pissiarilaungmagit.

2. Ipaksinatsangilarle Jêsub nâpkiningninga: 
272 (c) Nukapitsat nullêtullo imgerutinginik.

Tamainik pingortitsijoк,

Nâlegar Engelinut,

Tâmna kilangmit akKartor

Nuname innûlermat,

Ajorneк piginagole

Uvaptitun-ilaungmat.

3. Tâmnatauk sinnigvingminit

Makkipok, merngortormut

Ama innangalaurivor, Kâlauкрок immerukpor, Taimaktauk orâlalaukpor

Uvaptitun-idlune,

Illânelo kiægukpoк,

Illâne ajoкsarрок.

4. Tamanna erkaraptigo

Kuviasudlarpogut,

Nellopkotigalloarluta

Imâk oKautivavut:

„Inûsuktôlaungmigavit

„Ajornangilar mânna,
„Tautukôtsainaruptigit "Idluarsilerpogut."

5. Tagva timivut tarnivut Tunnivavut Jêsumut Aunganut tokkunganullo Inûlijaukovlugit, Tarniptingne kênaptingne TækkuksaulerKovlugo, Pinasuarnivut Jêsus Adsigijomavlugo.

\section{Mel. 164.}

Wer durch sein blutig's Lösegeld. *1249.

Kivgaujungnaititaumajoк Ajortunit aunganut, Sorairane tætteKartoк Jêsusib tokkunganik, Okpervigivlugo, Adsigilerlugo, Nâleklugo opigomut; Idluarsivok nellâgut.

\section{SAIMARTITSINERMU'T.}

684. Mel. 218. Attê, Jêsusiga! tussakit, Nullêtulle nagliktavit Angerviginiarmattille Ingminiglo tunnivlutik. Nutâmik aggaktit puttoggit
Tâpkonunga mânna issakit

Tamaita pitsartutikit

Illingnut ungadlarkovlugit

Pinasuatsainarkovlugit Perkojatit nâleklugit. 
(d) Niviarsiat uigasuillo imgerutinginik, 273

(d) NIVIARSIAT UIGASUILLO IMGERUTINGINIK.

\section{Mel. $151 \mathrm{i}$.}

Viel Gnade, Fried' und Freude. *1250.

1. Saimanernut Jêsuse

Tækkonârtiguttôk!

Tunitsivigittigut

Ullapirsautingnik!

Nertoromagaptigit

Aunârnît pivlugo

Iûlilauraptigut

Kannimaniptingnit.

2. Kattaksimaniptingnik

Ikpigidlarapta

Attoriakarpavut

Gûd Piulijivut.

Attijomagaptigo

Piuliklerninga,

Kennuaridlarpavut

Tâpsomangat mânna.

\section{Mel. 208.}

Ein gross Mägdlein drücke. *1254.

1. Nunamêniptingne

Issumagilavut

Pijarialik:

Jêsusib sângane

Nutakkatitulle

Takkomôrata

Sakrijâlerkovluta;

Taimaipatta ômattivut

Gûdib nâpkigivait.
2. Illiornek tamanna

Attutsiarlavut

Takkomôrata,

Gûb okausingita

Imak pingmattigut,

Nutakkatitut

Nuname ilungitut

Gûdib nâlegauvinganut

Pijuksaungilattauk.

3. Asserorsimanek

Sakkijâtsainartok

Tailugo tamât,

Âtæksarivavut

Inûlijiptingnut;

Ôma naglimut

Ômatine angmarpâ,

Illûnaita kanoêtut

Âkitsainarmagit.

\section{Mel. 22.}

1. Tiulijipta kaujimavait

Okpertut illiorningit,

Idluinitik pivlugit

Ômame sanikterijut.

2. Taimaitut illagênarpait,

Tuksiarvigikpatsuglo

Piulijaujomavlutik

Mannigorpait nâmaktomik.

3. Taimaglo tarnit timillo Jêsus kuviagingmatsuk 
274 (d) Niviarsiat uigasuillo imgerutinginik.

Ajoksarangamik sumik Ôma najorpait mânnakut.

\section{Mel. 79.}

Ihr lieben Jungfrau'n-Chöre. *1266.

1. Jêsus nertornautingnut Ariksulaungmitigut

Naglerkâraptignt:

Najulerangaptigit

Erritsainaraptigut

Torârvigênarlaptigit.

2. Perkojarnik tamainik

Illinniartitigut,

Ivsornaikovluta,

Tamât ômatiptingne

Gûb mamaigijanganik

Tækkuksaujokarkonnago.

3. Tamapta ômativut

Tunnivavut illingnut,

Igpigilerapta :

Idluitullinerub

Kiksartimmago inuk

Ôksilauktok Jêsusemik.

4. Gûd tokkungmat pivluta Piulijaukovluta

Idluiniptingnit,

Ômallo issumallo

Ârikpait adsiminut;

Katjârtine mannigorpait.

\section{Mel. 22.}

Das ist und bleibet unser Trost. *1268.

1. Mannigortauvogut, Jêsus Aungnut piuligaptigut;
Sangêtogut saimanernut

Najutsainalauraptigut.

2. Ômatiptingnut itterit, Innigitsainarlugillo;

Tarnit timillo saimakit, Tamainit serngnigilugit.

\section{Mel. 185.}

Jêsuse Kristuse Nâlegavut. Naglingniktorsôgavit, Pissiarigaptigut tamapta, Kejungme tokKogavit: Ômativut pigilaukit mânna

Ânianivit akkiksangænnut. Perkutigilutalo

Mâne inôtilluta.

\section{Mel. 22 a.}

Die Art des neuen Herzens ist. *1274.

1. Ômatib ablatsauningat Illinganinga sakkerpor, Nellâgotsainarninganut, Sorlo Gûb perkolaungmago.

2. Naglingniktor Nâlegarut, Ômamut tuksiarpavut, Idluarsinerme mânna Pigannitsainarkovluta. 
(d) Niviarsiat uigasuillo imgerutinginik. 275

3. Nâlegar ikkajortigut,

Illitariluarlutit

Tamât illinniarluta

Perкojatit nâleklugit.
4. Ômame missigivlutit

Imgerluta nertorlutit, Sorairatalo Kaut tamât, Uvlâmit unnungmut aglât.

\section{SAIMARTITSINERMUT.}

\section{Mel. 185.}

1. Jêsuse aungnut uigasuit

Pitsartutilaungmigit!

Nertornautingnullo ômatingit

Ubvalaungmigit, sângne

Nellâgut ivsornailerkovlugit,

Najornernelo inôKovlugit,

Issumalinginermik

Illangiutitilaukit.
2. Piuliklernerniglo tamainik

Opigosuktilaukit!

Nikkanarnerlo nâmagi-

gangne

Piojoringitikit!

AjoKsarmatta tukkisitikit

Aklujut nâpkigijauninginik;

Ôksitilaungmigillo

Pitakaloringnernik.

\section{(e) NULLIARET IMGERUTINGINIK.}

\section{Mel. $151 \mathrm{i}$.}

O Vater deines Sohnes. 892.

1. Atâtanga Ernerub Pairksijotôgavit, Anerner nầksaungitor,

Piuliklerte Jêsus,

Naglingnît nertorpavut

Opigidlarlugo

Kaut tamât nâpkiningnît, Nutângortinangne.

2. Nulliarêk ukkoa Kattititaukammêk,
Amuakik illingnut,

Kittorngarilugik;

Nâlegak naglingnernut

Igvit rellaksukik;

Tuksiarvigingmattit,

Tussalaungmigigle.

3. Inôniarkovlugik

Idluarijarnut,

Tokkungnut kiblidlakik

AdsigêKovlugik;

Mikserârnernik Gûde,

Ijekartidlakik 
(e) Nulliarêt imgerutinginik.

Passijæksaukonnagik Tarnekuttimekut.

4. Tuksiarnivut Gûde!

Tussalaungmigille

Gûb illagêngningane Perorluartikik,

Akkonaptingnêdlutik IllagêrKovluta,

Kuvianartovingmik

Tækkorârtinnata.

\section{Mel. 159.}

Herr Christ, der Du den Ehestand. *1284.

1. Nâlegarle! nulliarêk Pingorterkârangne, Sivorlerpâglo aggangnut Saimausilaurangne: Tagva kuviasukpogut, Mânna sujungaititigut, Kristumut illagênullo Adsiokovluta.

2. Timivut ômativullo Aungnut serparsakit, Innigitsainarlugillo Tamaita tarnivut, Uvlut tamaita nuname Inôlerta najornerne; Tamaita piniarnivut Idluarsitikit.

695. Mel. 230.

Unser von dem Hérn beglückter. *1285.

1. Nulliarêngnivut mânna, Nâlekamut ullapкosertar
Kilangmelo kellaksortak, Nangminer pingortimmago,

Aungminut sujungaitilugo Gûb sângane idluarpok;

Issumanganullo

Aiparêngniptingne

Perkovluta;

Gûdiptale illuptingne, Ajokertormijâtigut.

2. Kittorngavut uvagullo Nâpkigijæksaungitogulle, Ajortogut illûnane;

Taimaimat Gûb nâpkiningit

Sakkertut nulliarêngniptingne

Angijôvut illagêne;

Gûb Kaumaninganik

Id Tuarninganiglo

Pekarapta,

Mannigoijub

Najorningat

Nekkoksitidlarmattigut.

\section{Mel. 79.}

Der Heiland ist der Seelen. ${ }^{*} 1286$.

1. Jêsuse igvit tarnit

Annerimalaurangne

Issokangitomit;

Nâlegarivaptigit,

Erniangojogullo

Inôkovluta illingnut. 


\section{(e) Nulliarêt imgerutinginik.}

2. Ajornekangitotit Jêsus Kaujivaptigut, Aiparêngniptingne

Ubvartaugiakarapta Aungnut; Jêsus atterne Kattititaumagaptale.

3. Omat taktuglo igvit Nautsertutsainarangne, Kaujimavaptigut

Kivgaujungnaimangâpta Ajortunut. Tokkungnut Pitsartutilaungmitigut.

4. Aiparêk sivorlerpâk Kattangmanik illingnit Óminarsilaukpuk. Mânnale kattangnivut Pêjarangne tokkungnut, Okpertungortipaptigut.

5. Jêsuse aukpit kôktub Ikkingnit, kiblikligit Timivut tarnivut; Nulliarêngnivullo Âkiguk naglingnernut Perkutaugapta illingnut.

\section{9\%. Mel. 16.}

Jesu Christ, Du Haupt der Ehe. *1288.

1. Jêsu Krist! nulliarêngner Vvsornaitilaurangne Nâlegavut najornernut Nulliarêt saimakit.
2. Aunârnernut ômativut KiblitsainalaukittôK! Sângne idluarijarnut NulliarêKovluta.

3. Nulliarêngniptigulle Ajornerilauktavut, Aungnut pêjalaungmigille, Tuksiadlarpaptigit.

4. Mânnamit inôsiptingne Akitsainarmitigut, Kaumanernut Kaumartigut Sângnêtsainarkovluta.

5. Ômativut missuktikit Aungnut, piniarnivut Illa idluarijarnut Perkovlugit nellâgut.

6. Kittorngavut illunaita Kuviagidlartarnut Perorkovlugit; illingnut Perкutaukovlugillo.

7. Illerasukonnatalo Sângne, tikkimiguvit, Itterkattigilaptigit Kuvianartovingmut.

\section{Mel. 15.}

Wir beten an vor's Königs Thron. *1289.

1. Nâlekaptalo sânganut Mânna pâmangavluta Tuksiarpogut: aunganut Ubvartaujomavluta. 
(e) Nulliarêt imgerutinginik.

2. Ikkajortaujomavluta Illa sangêdlarapta, Nellâgut nutakkatitut Sângane - erkovluta.

3. Illuptingnele ajortut Kivgartorungnailugit, Pêjalaungmigit, timivut Nellâgut âkiklugit.

4. Ânertaulauktotit Gûde; Nakoridlarpaptigit, Sullele timeKaravit Timipta adsinganik.

5. Uviningme inôvogut, Ajorner mallingnago; Okperapta Jêsusemut Sapputivâtigulle.

6. Timivut tarnivut Jêsub Ivsornaitidlarmagit, Aungminut kiblidlarluta Illuvut aumatipait.

7. Jêsusib ânianganut Âkiktautsainarupta, Idluartomik pivogut Nulliarêngniptingne.

\section{Mel. 10.}

Vor Jesu Angesichte. 896.

1. Jêsub kaumasingane

Ijikita sângangne

Nulliarêlerupta

Idluarpor illâle.
2. Kanniname uvaptingnut, Nulliarêt taimaitut Najutsainaromavait, Naglimut tækkonârpait.

3. Jêsuse! innôsernut, Aungnullo âniarnut Inûlidlaraptigut, Missigitsainarlavut.

4. Tamaita piniarnivut Issumallo oKautsit Nellâgut illingalit Jêsusib adsinganut.

5. Kittorngiolerupta, Inoguilugillo Gûd idluarijarnut Ikkajutsainartigut.

\section{Mel. 185.}

Nulliarêngortitsijôgavit, Saimaijorlo Nâlegar! Kittorngavit kennuvigivâtit

Pilorigomavlutik; Aggangnullo puttosimajunut

Pikit, kattimajut ovanêtut Sakкervigilerlutit Nerriugvigingmattit.

\section{Mel. 114.}

o Jesu! Du Gott aller Geschlechter. *1278.

Jêsusivut nâlegarigaptigit Ômamut kennuvigivaptigit; 


\section{(e) Nulliarêt imgerutinginik.}

Kittorngavut illagilugittôk Kittorngarnut illaliulauktigut,

Saimanernik nungusuitomik

Issuanit angilauraptigut.

902. Mel. 166.

Kittorngavut piluarlugit
Perkovavut illingnut,

Tuksiarvigivaptigit

Jêsuse, tussartigut!

Aungnut serparsarniakit,

Ungatikit illingnut;

Illûnatik aglissiglit

IdluarijarnuttôK.

\section{SAIMARTITSINERMUT.}

903. Mel. 83.

1. Sokrosijuitikit

Jêsuse pitsartunernut,

Najornernullo mâne

Timingillo tarningillo

Nekkoksijaukovlugit

Tuksiutigivavut.

2. Ivsornaigannertikit

Saimanartitilugillo

Inuit tækkoænne

Pitsartutilaungmigillo,

Idluaringitarnik

Tækkotiniarlugit.
3. Taimailiorkovlugit

Nâlegak ikkajulaukit!

Kajungersainingillo

Igvit nâmaksitilaukit,

Sillatojutitullo

Arvertartikit mâne.

4. Jêsus saimalaukittôr

Uvlome angajoxâvut

Ikkajutsainalaukit,

Uvaptingnik pairksingmatta;

Pitakaloringnermik Illangiutitilaukit.

(f) UIGARNERIT IMGERUTINGINIK.

204. Mel. 79.

O Jesu, unsre Freude. 898.

1. Kuviasugviksavut

Mannigorvigijarut,
Kiksarviksaptingne:

Jêsuse pitôjotit,

Tamaita kittorngatit

Naglimut mannigorangne. 
2. Nellekangitor ômat, Naglingnermik tættætok, Nertornartôjotit! Jêsuse issumangnut Illingatsainarupta Tamaine nâmaksivogut.

3. Magguk attausiojuk Avikunik illingnut Kiblingnarpoк illa, Taimaidlarmat illuvut Kiksarpok, unganermut Aipaulauktok angergarpat.

4. Jêsuse naglingnernut

Ômame angijomik Mannigorpaptigut: Pituarigaptigit Najutsainarpaptigut Inutôgapta nuname.

5. Kennuvigivaptigit: Jêsuse mannigukit Tamaita kiksartut; Najukit, næglingnernut Kuviasuktilaukit, Uigarnerit tamaita.

705. Mel. 14.

$\mathrm{O} \mathrm{Du}$, der Wittwen höchstes Gut. 900 .

1. Jêsuse, uigarnernik Mannigoijotit, Kuviasuktênartigut Tokrungnut aungnullo.
2. Ômativut mannigukit, Nagliktotôgavit; Kuviasugvigivlutit Kujalerkovluta.

3. Sulle akkunera mâne Kugviorviptingne, Inôguptalônêt, sângne Itsainalaungmita.

4. Kiksartut tamaita sângne Jêsuse naglimut Mannigotsainarpattille, Ikkajudlarlugit.

5. Ômatit kugviorningit Igvit kittipattit Kiksarningillo pêrlugit Sattutidlarpattit.

6. Inôniarviksavullo Okitipat mâne, Angergarviksar pikpallo, Aijomârpat tarne.

7. Taimaimat tunnidlarpogut Uvaptingnik mâne, Jêsuse tessiornernut Uvlut tamaitale.

\section{\%06. Mel. 26.}

Halleluja, gelobt sei Gott. *1310.

1. Hallêluja! nertortaule Gûd, nâpkiniktor uvaptingnik,

Uigarniojogut, tokkomut Nagligilaungmattigulle. 
(f) Uigarnerit imgerutinginik.

2. Ômatinga tættædlarmat Nâpkigosungmik, kiksarnivut

Kugviornivullo tautungmagit

Mannigotsainarpâtigut.

3. Kemmangniangilâtigut Nâlegapta, tamattominga Kaujigapta serridlarpogut Pattangaitigigaptigo.

4. Omamelo naglingninga Missigigamitsuk tarnipta Nuttakkatut opigidlarpât, Inôsek okitimmago.

5. AjoKsatsainarnivullo, Killanârutigidlarpavut Ungagivlugo nutakratut Missigiganneromamut.

6. Kanoktôrle ômativut Jêsusib naglingninganigle Illumertaulit, uvaptingnut Nertortautsainarkovlugo.

7. Tâpsomangat taimaitomik Pititaujominar tamâne Tamaptalo najorningane Kuviasutsainarkovluta.

907. Mel. 16.

Welch ein frohes suisses Leben. 903.

1. Omame kuviasungneк Unêt sakkertinnago,
Angijomik nekkorsiner Gûd inôvigivlugo.

2. Aiparut tækkuksaujungnaipat

Jêsus tækkonârlugo.

Ikkingit nagvâruptigik

Nekkorsijaudlarpogut.

3. Uigarnioniptingne Gûde ikkajortigut Attit nertortaukovluga Ungamut uvaptingnut.

4. Asserutsijut issumat Pêjartaulit tamaita Kiksartitsituinarmatta Nekkorsinatsangilat.

5. Kuviasugvituavut Jêsuse pitôlaurit, Taimak tokкoviksaptingnut

Ungagidlarlaptigit.

\section{Mel. 167.}

Ruhm und Preis und Dank. *1314.

1. Jêsub Kristusib attinga

Nertornak opigomut,

Uigarnerit tâppsoma

Mannigutsainarmagit,

Taimaktauk illiarsuit

Ôma nâpkigosumut

Pairitsainarmagit illa Inoguidlarlugit. 
2. Tâpsoma nâpkiningninga Sokкosingilak illa! Kittorngavullo tamaita Nagligivait tâpsoma, Ajoksaluarangatta Mannigorivait tagva, Kugviolerangaptalo Najutsainarpâtigut.

3. Uigarniub Jêsuse Pituariguniuk Hannatut kattimavingme Tuksiarvigivlugo:

Killanârtorlo Jêsuse Tækkojomavlugole, Erkilugolo takpâne Mâne Kuviasukpor.

4. Uigarnek Jêsusemik Ôksijok illumine Illanâkarninganiglo, Tessiortaulerame Tâpsoma Anerninganut, Illumertaumagame Jêsusiblo ijingminut Tessiutsainarmago.

5. Taimaitub nellâgut mâne Kaumaksarutigivâ Unêt aipairutiylune Ômame serrigivâ. Kina taimaitsomangitor? Nutakratut Jêsumik Tækkonâromatsængitor Nekkorsijomanane?

6. Uigarnerit tamapta Idluarijanganut
Taimaglo inôniarta!

Nekkoksinartotôngmat. Okpernerlo naglingnerlo Tættekarnek Gûdemik, Sakzijârlit uvaptingne Perkutigingmattigut.

7. Merngoêrservigilavut Gûd Kuviagilugo; Êgangavigilavullo Tættituarilugo.

Uvlorme unnuamelo Pitilavut inôser Ômunga, missigiluta Ullapirsautinganik.

F09. Mel. 205. Amen Ja, Halleluja. Herr. *1316.

Amen illa Hallêluja! Gûde tættigivaptigit, Jêsuse naglingnîlle, Pivluta aunâlauravit, IpaksinatsængitoK Idluarsinadlarmat, Tagva kujalinermut Opigidlarpaptigit.

\section{F10. Mel. 79 .}

1. Tættigilaptigille Jêsusivut! tamapta Tuksiarpaptigit: Serparsartigut aungnut, Illuvut Anernernut Najorlugit, innigikit. 
(f) Uigarnerit imgerutinginik.

2. Nâpkiniktorsôgavit, Sangêtut illûnatik Tikkivigivâtit ;
Jêsu Krist, Anernernut, Tokkungnut ikkingnullo Uigarnivut saimakit.

\section{SAIMARTITSINERMUT.}

\section{Mel. 37.}

Habt eure Lust am Herrn. 904.

1. Jêsuse saimakit

Uigarnivut,

Tunnitsivigikit

Ômattingita

Pijomajanginik,

Tættigingmattit
Nertornartôgavit

Pairilaungmigit.

2. Nerriungninginit

Piluartomik

Nâpkigilaungmigit,

Saimarlugillo,

Piloriktilaukit;

Inôsingine

Kuviasuktikit

Uvlut tamaita.

\section{NÂLEKAB ILLAGÊKTULLO KIVGANGINIK.}

\section{Mel. 228.}

Geist, Seel' und Leib ist Dir geweiht. 906.

Tarne time Gûdiptingnut Tammarmik tunnidlarpavut,

Âxilikik Jêsuse, Âniajotit pivluta Tækkolauraptigit, tagva Inôjomaerpogulle Uvaptingnik; kissiane Sangêtogut opigomut Kivgartorlaptigit mâne.
213. Mel. 70.

Wir sind nur dazu. 915.

Inôvogulle

Perkojaumagapta,

Nâlegavulle

Orautigilugo ;

Angerpogut taimaitsomamut,

Tâpsomalo anneringmattigut.

214. Mel. 206.

Lamm, Lamm o Lamm. 916.

1. Nâlegara :,; nagliktara

Tokkojotit 
284 Nâlekab illagêktullo kivganginik.

Uvanga :,: pivlunga

Pigijungnaipara:,: tarniga, Jêsub ikkingita aungata mânna

Pigingmatsuk :,:

Pissiaringmago :,;

2. Nâlegitse :,: illûnase, Gûdiojok

Nunamut:,: tikkipor;

Ajornivut illa :; pêrpait Aungminulle, tokrogame Piulilugit :,:

Inuit tamaita. :,:

715. Mel. 185.

Einig's Herze, das soll meine Weide. 917.

1. Kissivit nagliktara Jêsuse! Pituaridlarpagit;

Illingne inônialaulanga, Arvertarlunga sângne;

Pivlunga kuviasurovlutit, Attulaungmingalo issumangnut:

Ikkingnut pairilaunga.

IdluarserKovlunga.

2. Merngoêrsertuksaumangârmale

Piniarlungalônêt,

Igvit nellojungnaitidlarparma:

Tamarmik ajungilak.

Najornernele pilorikpunga,
Pikkarludlarpakalloarama Ajoksadlarnimnele Tættigivagit illa.

\section{Mel. 26.}

Ein inniglich vergnügtes Herz. 920 .

1. Tunnitsivigittiguttôk Ômatinik kuviasuktunik, Aungnullo ônartitaujunik, Nekkorsititsainartigut.

2. Najutsainartigut illa Perkojarnik piniarupta, ToKkungniglo orâlagupta OKausiksakartitigut.

\section{1\%. Mel. 155.}

Mache uns zu Deinem Dienst. 923.

1. Kivgartorkogaptigut Inungne annerijarne Illagêne

Kaikojomadlartarne Kilangmut, Attaruitilauktigut! Kamatsialerkovluta Piniaræksaptingnik.

2. Illa Jesus, tamaile, Tukkisititsainartigut Inurotitit Aglikojomavlugit Pigavit: Illangiutitilaukit 
Attunit piuliklernernik, ... Kuviagilugillo.

\section{Mel. 58.}

Lass keinen unter uns träge sein. 925 .

1. Erkeasujuititigut,

Kajungersarlugit tarnivut Taimaktauk timivut,

Perkojatille

Nâniatsainaromadlarlugit

Kuviasumut.

2. Kattersortatit sapputikit, Pairksinerniglo opiguktut,

Tungatinangnelo

Timekut, tarnekut;

Illûnatiglo piniarningit

Nellâgôrlit.

\section{Mel. 141.}

-Unsers Lammes Wunden. 930.

Jêsusib. ikkingit

Angmartak senneranga

Inôvigilerpait

Sangêtut kivgangita;

Pituarivaillo

Piniarunik,

Nokkarangamiglo

Sullinermingnit.

\section{\%20. Mel. 37.}

Wir sind dem blut'gen Lamm. 928.

Saugârsuk ikkilik

Kivgartorlugo,
Pijuksaudlarpogut

Inôsiptingne;

Arvertarniptingnut

Kikkarniptingnut,

Adsigêdlartomik

Nakorilugo.

\section{Mel. 166.}

Nimm mich von neuem in die Hand. 936.

1. Aggangnut pitsartujunut Nutâmik tigulaunga! Tâpkonunga illûnaita Sâlakartidlarangne. Uvangatauk tessiunga Aprotimne tamaine, Pairrsinernik nâmaktomik Kujalidlarkovlunga.

2. Illûnamnik tunnivunga Illingnut, Nâlekamnut, Nâmagijarnullo illa Kivgartoromavagit. Igville sapkotailiuk Tarnigatôk aggangnit, Illa uvlâmit unnungmut, Napkigitsainarminga.

\section{Mel. 14.}

Wie selig ist Dein Hausgesind'. *1366.

1. Kivgavit nertoramitit Jêsus, pilorikput Tattigênarangamitit Pitsartutitauvut. 
286 Nâlekab illagêktullo kivganginik.

2. Nâlegak opigidlarpât

Pitsartutimmagit,

Kennuêlutik nertorpât

Sullitsainarlutik.

\section{\%23. Mel. 29.}

Ein Führer muss vor andern Gaben. 939.

Assiminik tessiortuksar

Nangminer pijariakarpok

Ômamik nâletsiartomik;

Gûb inungit pairilugit.

\section{Mel. 10.}

Wollst Deine Diener salben. 941.

1. Kivgatun-ilauravit

Kivgatit nekkorsikit

Illagêt akkorngænne;

Tussartigut Jêsuse!

2. Ômativut serrilit

Tarnivut ônarsilit

Kibliktaunerlo aungnut

Tækkuksaule kênakut.

3. Kanoktôk! illûnâne,

Nellâgôtsainarluta,

Sakkijârta tamainut

Jêsub kivgangititut.

4. Gûd kivgavit pijangit

Idluarsitsainarlit!

Illûnâne tamapta

Attaruerkovluta.
5. Tarnivut timivullo

Nekkorsitilaungmigit!

Mannigulaungmittigut

Kiksartitaulerupta!

ซ25. Mel.'-184a.

Nâlegar pitakaloringnît

Piniutigilauktatit

Inûlernernut tokkungnullo

Illangiubvigilakka.

Ômatiga tamakkonunga

Nekkorsijautuinarpat,

Serrilunga kivgartorpagit, Tækkokarnanga illingnik.

\section{F26. Mel. 185.}

Hast Du mich zu Deinem Dienst. *Anh. 240.

Kivgartorkogumga Nâlegara,

Iglungne, illagêne,

Sunamik piniartuksauguma,

Kennuvigidlarpagit:

Sungertûtekartailiti-

launga

Pitsartunimnik; saimarsainîlle

Tættigitsainarlago

Sullitsiarkovlunga.

72\%. Mel. 189.

Von neuem sei Dir unser ganzes Leben. *Anh. 243.

Nutâmik illa inôtsivut tamât 
Pigijavullo tunnijaulaulit Illingnut, ômativullo tamâne

Kivgartorkojinernut attukit.
Tokkolauravit,

Kivgartorlutit

Opigosumut

Serrigilavut.

KRISTUSIB NÂLEGAUVINGATA OKAUTAUNINGANIK TILLIJANGINUT KIGLIGIUDSININGINULLO NUNAME.

728. Mel. 58.

Wollt ihr Posaunen der Gnade sein. 957.

1. Okautigijomagupsiuk Saimaninga, pigerkârsiuk, Ajorungnairitse Jêsub aunganut; Kingorngagut oKautigisiuk Illapsingnut.

2. Illagêt illûnânemiut Illagivlugillo, inungnut Okaromavogut: Jêsub aunganut Kivgaujungnaititaujuksaudlarput Ajortunut.

3. Tamaigiguptigo Jêsuse Pivluta tokкojok kejungme;

Illa naglingnermut Aunâdlalauktor Missigijungnairuptigo, tagva

Kappianar.
4. Illagêktut inôtsiarput Êgaramik nagliktimingnut,

Jêsub ikkinginut

Ajorungnaiput, Âniangane merngoêrserput

Sorairatik.

\section{Mel. 39.}

Hier hast $\mathrm{Du}$ uns alle zu

Deinen. 958.

Mânetogut perkojatit malliklugit,

Piniarrogaptigut nâlekpaptigit

Perkojatillo illûnatik nellautsivut

Tættigidlarlutit piniaruptigik. 
288 Kristusib nâlegauvingata okautauninganik

\section{\%30. Mel. 70.}

Preis sei dem Blute. 960 .

1. Jêsub aungminut

Inuit tarnaita

Sillaksoarmiut

- Kiblikpait, opinar!

Ômunga ônarsilingitut

Tarnit, illûnatik tokkungavut.

2. Naglingningane, Merngoêrserpogut,

- Piniarnermut

Kajungertipâtigut.

Ôlsinermik tamattominga Tukkiseкattalaukpogut illa

\section{\%31. Mel. 79.}

Lamm, schenk' mir Deinen Segen. 964.

1. Nâlegara saimaunga!

Nane arvertaruma

Inuit nellîne,

Kivgarnik taijaulunga,

Taimak piniaruma

Nekkoksititsainarminga.

2. Pivlunga Kennuvagit Assimnik nâpkiguvit Nachogiminanga. Missigitinga, Gûde, Naglingnermik illumne; Najornernut nekkoksinga.
3: Aulik Piulijivut

Illitarivaptigut

Sulle ajortogut,

Taimainapta illingnut

Torkoênarmitigut

Tamâne inôtilluta.

\section{1 a. Mel. 155.}

Herr und Haupt Immanuel. 967.

Nâlegar Imanuêl,

Ômat saimaneliksôjor!

Naglingnivît

Kiblikpâtigut illa;

Tuksiarnît (Joh, 17.)

Atâtangnut aikârtinnak

Nellautsijariakarpok

Sillarsûb naggatânut.

732. Mel. 95 .

Jesu durchgegrab'ne Hände. 969.

Aggane puttoggit Jêsub Sorairane tammartunut Issakpait illûnainut, Nellojut sunatuinait Piulijaujomadlartut Aitipait iglominut.

\section{Mel. 70.}

Wol auf, ihr Hoiden. 1109.

1. Piulijiga!

Tuksiadlarpagit 
tillijanginut kigligiudsininginullo nuname. 289

Inukattikka

Naglimut saimakit,

Ikkingnullo tessiormigit

Tamaitale pilorekovlugit.

2. Aniagavit

Kiæguklutillo

Auktun-itunik,

Erkaumadlârapko,

Ômunga sapputilaungminga

Ajortunut kajungerkonnanga.

3. Attê nellojut

Tuppaleritsele,

Pitsartujomut.

Piulijautitse

Jêsusib mânna kaikungmase

Allarniarsigik ajornise.

\section{Mel. 230.}

Piulijivut tamapta

Ômamut nakoridlarlavut

Tagvunga âlaungmattigut,

Itsar sulle ajornipta

Kivgaringmattigut, tâpso-

ma

Kivgaujungnaitipâtigut;

Kujagidlarlavut,

Nagligilugolo

Sorairata,

Ânianga tokkungalo

Erkaumadlarlavut illa!
\%35. Mel. 189.

O Geist, Du Prediger von Jesu. 968.

Anernek! Jêsub ânianinginik

Okâlajotit, tuppaktilaukit Ômatit unnuktuttôK, okautsivut

Ôminga, tussartaulerkovlugit;

Perkovlugolo tussunerminik:

Sulle tarninik

Sillingilar.

\section{\%36. Mel. 155.}

1. Ananaujor Nâlegar!

Illa ânanaugivagit,

Auktogavit,

Ikkitillo aunârtut

Tamaita

Inûlijigidlarapkit,

Kunnidlarpakka kugvinut

Piulijigigapkit.

2. Sumik okalerlangâ?

Erkaigama ômatimne,

Nellojune

Tarsiudlalaurama,

Tamaunga

Jêsuse aitilauramga

Aungnullo: eprêjarlunga,

Najutsainalerparma.

\section{ร3\%. Mel. 166.}

1. Tamainik pingortitsijor Akrarlune rilangmit, 
290 Kristusib nâlegauvingata oxautauninganik

Inûlilarmelo pijor

Nunamut naglingnermut;

Tokкotæksaudlalaurapta

Ajornivut pivlugit,

Gûmut ullapKoserluta,

Pivluta toккоlerpoк.

2. Gûdib ningausinga illa

Ikpigivâ pivluta,

Ingminik tunnilaurame

Pitlaræksaukonnata;

Sajulerpor kiksarnermut

Tarnimigut, tokkomut

Kappiaktoadlarnermut

Aungmik kiægulerpor.

3. Pititaungmat unnuame

Ajortut agganginut,

Tâpkoa tigludlalerpât,

Kênanga ketserarpât,

Mittautigidlarlugolo,

Unertôtigidlarpât

Ajortullijorlo sorlo

Tokrotæksangortipât.

4. Sorsuktut annoraijarpât,

Timinga ivsornaitok,

Issakautinut killerpât

Naiperkotiginago

Orpinut kakkilautinut

Niakoserpât illa,

Killerlugo taimaitomut Âniatidlalerpât.

5. Taimak âniatitaungmat Serlexititauvlune

Kejungmut kikkiæktorpât,
Tagva kovivâ aune;

Inôtsine tunnivlugo

Anernangilerlune

Mânnale ajortorijut

Inôtituarivât.

6. Illuvermullo illivât;

Uvluk pingajuangne

Makkipor ômalarmelo;

Mânna appertsorpâse:

„Naglikapse taimak, mân-

$$
\text { na, }
$$

„Tunnidlaromavise

"Illipsingnik? inôvluse

"Idluarijamnulle?"

7. Atâtab tallerpiane

Mânnale jksivajok, Ama ânanauvingmine

Nakorênadlartaujoк,

Ullernaigidlarlavuttôk,

Nagligilaungmattigut,

Naglingningat nungutip $\hat{a}$

Pivluta torkogame.

8. Nâlegak opigosumut

Kujagidlarpaptigit,

Tikkinaptigut nunamut,

Pivluta toKkovlutit;

Tagva tuksiarpaptigit

Ômativut pigikit,

Ubvarlugit ajortunit

Aungnut arklingnartomut.

938. Mel. 166.

Die Heerde freut sich, treuer Hirt. 972.

1. Pairksijok, illagêt tagva. Ômamut serridlarput, 
tillijanginut kigligiudsininginullo nuname. 291

Unnunersaulerangatta

Piulijatit aungnut;

Kanoktôx illagilugit

Âniat nertorlavut!

Aullo okautigivlugo

Illagêt tungavingat.

2. Taimaimat, tillijatittauk Aularput nunalingnut:

Tarniggit ivlerigangne

Pijomadlarangnelo;

Merngortornak nunamiut

Unangmitsainarangne,

Ikkivit Kaumaningænnut

Kaumaksartaukârtinnagit.

\section{ซึ9. Mel. 121.}

Ach säh' doch jedermann. 973.

1. KanoKtôk tamapta

Mallugijaulerta,

Inungnut tamainut

Perkojaumagapta

Tarnivut timivullo

Tunnimariklugit,

Piulijivut

Kivgartorlugo.

2. Jêsuse tokkungnut

Aulingnut ikkingnut,

Sennerkab ikkinganut,

Aungmik kiægungnernut

IllagêktoKotitit

Pairilaungmigit,

Sapputilugit

Tikkiviksarnut.
740. Mel. 79.

Geht, Zeugen, Jesum malen. 974.

1. Kigligiudsijose

Okariartoritse

Inungnut tamainut:

Jêsuse âniangmat,

Kejungme tarningêrmat;

Piulivait tokkominut.

2. Aulik Piulijivut

Tamât kaujivaptigut

Ajorapta sulle:

Taimaimalle tokkungnut

Inûlitsainartigut,

Kivgartorkogaptigulle.

\section{Mel. 166.}

Ein Lamm stellt sich zur Zahlung *1423.

1. Saugârsuk tunnidlalauktor

Ingminigle tokromut;

Piulijomagamigit

Tamaita pattangajut;

PiulijiksaKangimat

Assianik nuname,

Ikkingita aunârningat

Piulijautigivât.

2. Mânna aularpogut tagva

OKarluta inungnut

Pijuksaungmatta Jêsusib

Iglunganut nellâgut;

Uvaptigut appertsorpait:

Kaijomangimangâta 
292 Kristusib nâlegauvingata oкautauninganik etc.

Akkiktaulerlutik ôma Aniadlarninginut.

\section{Mel. 29.}

Kein Volk noch Sprach'. 979.

1. Tamainik pingortitsijotit

Jêsus nachogitsangilattit

Nellìt sillaksoarmiut

Tamaita nâpkigivattit.

2. Taimaimat tussumarikpogut

Sulle nellojut unnudlartut Okautilugit illingnik,

Piuliklerteojotit.

742 a. Mel. $151 \mathrm{u}$. (ubvalo g.)

From Greenlands icy mountains. 934.

1. Karâlit Kakkanginit

In đia tikkidlugo,

Afrikab nunangine

Kiaktakartune,

Nellojune tamaine

Niplertokadlarpoк:

„Kaititse, pêrsititse

„Kelaumgujaptingnik!“

2. Kaumaksartaujogulle

Takpângat kilangmit

Nellojut tâksiortut
Nâpkigidlarlavut!

Piulijautiksamik

Tussarnidlartomik

Inuit illûnaita

OKarvigilavut!

3. Nunanut illûnainut Jêsusib attinga Nertornartok âtaule Annorinut sorlo!

Siamartitaulaurle

Opigijauninga

Nâlegaumariklune

UtterKârtinnago.

743. Mel. 58.

Lass Deine Kirche je mehr. 981.

1. Jêsuse! illagêktitatit

Unnuksivalliatilaukit

Nertornadlarnernut,

Aukpit inuit

Okpingitut sâgiartiligit

Illingnuttôk.

2. Illagêngnivut saimauseruk,

Omativullo tunnilaukit

Ullapirsautingnik;

OKautsivullo

Illingatsainarlit illa tamaine

Issumangnut. 
(a) Nangminer pivlune assivullo pivlugit. 293

\section{KENNUNERMIK，TUKSIARNERMIGLO TUKSIARUT- SINERMIGLO.}

(a) NANGMINEK PIVLUNE ASSIVULLO PIVLUGIT.

\section{Mel. 136.}

Das ist mir lieb. 1000.

1. Nakudlarpor

Gûdima kennunikka

Nachogitsænnagit tussadlarmagit;

Saimarsaijub, illa naglingniktub

Tulssiartut ajæktorniængilait,

Ajoksartut ajortuggit nagligidlarpait;

Taimak illitarigapko :

Nakudlarpox.

2. Sorairnanga

Erkaumajovakka

Taikattarlugillo angerningit;

Nellâgôrningalo tættig:va$\mathrm{ra}$,

Nagligosungningalo erkarapko,

Kujalimut Kugviornermut nertorpara;

Saimaninga missigivara Sorairnanga.

3. Nerriuktor

Okperidlartaminik

Tækkojomârpor, piloridlarlune
Imâk pivor: piulijauvunga,

Issuanit Gûb nagligidlarpânga;

Kugvikka pêrpait, ijukkavêrutivânga,

Mânnale tækkuksaungitor

Pijomârpok.

\section{4 a. Mel. 110.}

Dir dir Jehova will ich singen. 995.

1. Jehôva! imgervigivagit

Nanele illiktut GûderarKa?

Nertordlernikka kailerlugit,

Pitsartutilaunga Anernernut!

Perkovlunga Jêsub attingane

Tâpsomûna kuviagigangne.

2. Atâtak! amuanga Ernernut,

Ernivîllo pilinga illingnut; Anernît illumnênalaule, Issumamnik aulatsitsainarle, 
294 (a) Nangminek pivlune assivullo pivlugit.

Ullapirsaumik Gûmit pijomik

Ômatimne pekainarkovlunga.

3. Anernerpît tuksiulinga OKauserijaujungnangitomik.

Tâpsomalo Kaujitipânga Kittorngarigamga Jêsuseme;

Tâpsomane Atâtagigapkit, Tagva Abbamik tailerivagit.

4. Anernerpît 'tuksiartitima

Issumangnik nellautsitipânga;

Tussarpattillo Kennunik$\mathrm{ka}$,

Jêsub attingane Kennugama;

Tuksiarnimnit anginersamik

Pititsivigitsainadlarparma.

5. Jêsub attingane $\overline{\text { Kennu- }}$ vunga,

Akkisartortima, Gûd, illingne!

Tâpsomane ahailângovut Pângat kennunikka okpernerme.

Pitsainalaurit Kujalinermik,

Piloriktigamga angijomik.
ข45. Mel. 155 .

Mein Versöhner Jesu Christ. 998.

1. Erkarapko tamanna UllapirsaijoK Kristuse, Jêsusiga

Kanor piniaramga,

Taigamga

Gûb kittornganganik, mânna

Opigomut pâmakpunga Kutsaservigællutit.

2. Atâtavit sângane

Tuksiarutilerungne

Kittorngatit

Tagva erkalaungatauk:

Aunârnît

Ikkitit nertorangapkit, Sagvaikovlugo uvamnut Kanok pijuksaunimnik.

\section{Mel. 218.}

Herr Jesu, höre unsre Bitten. 1006.

Tuksialerupta illingnut, Tussartigut, saimarluta; Ullapirsautingnik tarnivut Illumertikit Jêsusîk! Ungagijivit illûnatik Mânna ômamigigamitit, Erkaumatsainarpâtittauk; Mallungnarsiluta tamapta Jêsusib arvertarkattipta Avatine pairingmagit. 


\section{(a) Nangminek pivlune assivullo pivlugit. 295}

947. Mel. 36.

Du Ha upt des Leibes Deiner. 1007.

1. IllagêktoKotivit niakungat Uvinekattitit tækkonâlaukit

Nakorivaptigit kujagilutit Sêrkortorluta.

2. Ikkingne Gûde, innilangajogut

Ôgaptigo tokkovit pitsartutâ;

Nellopкotimut kennuvogut sầngne

Saimarsainernik.

3. Jêsus ittersimajotit kilangmut

Inukotitit tuksiutilugit,

Sorainiarnæktôk nagligilugit

Erkarlugillo.

4. Illagêktitatit aglissitikit Tungatilugit aungnut toKKungnullo;

Attît nakorijaule uvaptingnut

Illûnaptingnut.

\section{Mel. 79.}

$\mathrm{Du}$ grosser Hoherpriester. 1011.

1. Kennuvogut illingnut

Pairksijor illagênik, Kajungersartigut,
Tuksiatsainarluta,

Naglingnernut illuvut

Ônartikit saimanernut.

2. Siutivut angmakit Tussatsiarkovlugit Iilanâkarnernik ; Orâlaleraigupta, Sumiglônêt pigupta, Pitsartutilaungmitigut.

3. Saimarlugit tarnivut Jêsus, illanârimut Timit innigikit Kaujitilaungmitigut Sillaksûb inunginit Annerijaumaniptingnik.

\section{Mel. 79.}

Er geb' uns munt're Kehlen. *1474.

1. Kajungertilauktigut Tattamnartullinernik

OKâlakovluta, Perkojarnik tamainik Nâlektilaungmitigut Nutakkatitut tamapta.

2. Illagêt tækkonâkit!

Pijariakartanginik Tunnitsivigikit; Tessiutsainalaukit, Kennuningit tussakit, Nekkorsititsainarlugit.

3. Pakkoa illagivlugit Nertutsainarlaptigit. 
296 (a) NangmineK pivlune assivullo pivlugit.

Opigilutillo!

Ittikkavit sângine

Kujagidlarlaptigit,

Uvaptingnik pairksigavit.

\section{Mel. 11 a.}

Ach mein blutig's Lämmelein. 1013.

1. Aulik Gûb saugârsunga

Illagêktorotitit

Pitsartutikit, tamât

Ungagitsainarmattit.

2. Najutsainarmitigut.

Illanâkadlarnernut,

Missuktikit tarnivut,

Aungnut kovimajomut.

\section{\%51. Mel. 119.}

Herzens Herr. 1015.

1. Nâlegan :,:

Tarnivut tunnivavut

Illingnut perкutauvluta

Nâlegomavaptigit;

Kissivit ungagilutit

Pituaritsainarlaptigit

Tamapta :;:

2. Jêsuse :,:

Ikkajutsainartigut

Piniarniptingne tamaine,

Tikkitauniptingnelo

Kiksarnartunut nuname,

Kaikojiniptingne inungnik

Atterne. :;:
252. Mel. 114.

Unendlicher, dess Jahre. *Anh. 197.

1. Nâlegavut! ipaksinangitotit,

Nelliutune tamaine illa,

Saimailutit naglingniojotit,

Issumangnik piniarungnartotit,

Opigilaptigit, illagêllo

Perkojaulit saimanernut. mânna.

2. Naglingniktok! kennunivut tussakit

Attortavut illagêt pivlugit,

Annerilauktatit perKutingnut:

Illingatsiarningit tautuklavut,

Aggangnullo ânanausitikit,

Kuviagijarnut erkovlugit.

\section{ชรอ. Mel. 184 a.}

Herr Jesu, thu' nach Deiner Gnade. *Anh. 205.

Nâlegak Jêsu, saimanernut

Tamât tuksiarniptingnit

Tunnitsivigiluartigut;

Arvertartimitigullo

Opigukluta aprotingne

Orautsitit malliklugit; 
(a) Nangminer pivlune assivullo pivlugit. 297

Taimak akkonaptingnêlaurit

Tamapta najorlaptigit!

954. Mel. 230.

Sprich zu unserm Flehen: Amen. *Anh. 213.

Kennuniptingnut Jêsuse, Ahailâlaurit, tagva attit,
Naglimut kujalimullo Opigijomadlarpavut. Nâletsainaromavlutillo Illûnâne inôsiptingne Ikkitit aunârtut Nênituinakit

Tarniptingnut, Tagva mâne - najornerne

Kilak illa ôgêrpavut.

\section{(b) ATTANNIVULLO PIVLUGIT.}

755. Mel. 97.

Wir fleh'n auch dankbar allezeit. 1016.

1. Erdlingnartor attannivut Tuksiaruparut sângne, Jêsuse nagligigangne. Nekkorsiuk ômatinga Inukotingillo illûnaita Ullapirsautekartilaungmigit.

2. Aggaktit puttortaumajut Tâpsomunga issalaukit, Pairilugo sapputiuk Tamainit piulilauruk, Sullijangillo perkojangillo Idluartut nâlektautsainarlit.

3. Taimak sulliarijane Nâniarpagit nuname, Takpaunga sângnut pilerpat
Pijangit akkilerungne, Idluarsitilauruktôr Gûde, Illaliorlugolo kilangme.

\section{Mel. 132 a.}

Gib mir ein recht gehorsam Herz. 1019.

1. Gûde nâletsiartomik Ômaterartilaunga. Kittorngatit perkogangne Attannek nâleklugo. Illingnut tillijaugamik Attannît, kamakovlugit Inuit illusinginik.

2. Perajaluktut tamaita Sâtilaukit illingnut; Ikkajortigut tamapta Nertoromagaptigit; Attanniojut aggangnut. Tessiokit inôtsemut Pâne nâksaungitomut. 


\section{IMGERUTINIK UVLÂKUT.}

\%ร\%. Mel. 205.

Jêsuse, attê mânna

Sinnimnit tuppalerama

Najunga ômatimne, Ikkitit missigilakka, Mâne inôtillunga

Tautukôromavakka;

Illingnulle aiguma

Kemmergojomârpakka.

\section{Mel. 22 a.}

Des Morgens, wenn ich früh. 1029.

1. Unnukpat sinnigomaguma Uvlâkullo makkikuma Erkarpagit Jêsusiga, Sapputijaujomavlunga.

2. Aggangnut puttomajunut Tessiunga nutakkatut, Aularvimne illûnâne Aiparitsainalaungatôk.

3. Jêsusiga, aut illûnât

Pivlunga kovisimangmat, Ubvalauruk ômatiga, Sujungaitsainarkovlunga.

4- Uvlâkut tuppaleruma, Taimaktauk sinnileruma, Illingnut ârlorpakpunga Saimausertaujomavlunga.

5. Uvlome pairilaungminga, Illingnit tammarkonanga,
Illanâtut najulaunga Kuviasudlarkovlunga.

ชอ๊ 9. Mel. 151 i.

Man steht aus seinem Schlafe. 1067.

1. Jêsub najorningane

Mânna makkipogut,

Taimaimat pitlartaunek-

Sivôraginago:

Uvloksiortitigut

Jêsus najornerne, Illagilaungmitigut

Uvlâmit unnungmut.

2. Inûb ajorninele

Kristusib aunganut Tokkotaukogunigit, Gûb sângane tagva Inôtsainaromavok;

Jêsuse kênarnut

Tækkonatsainartigut

Kaumaksarlutalo.

\section{Mel. 68.}

1. Illagêt illa,

Kuviasulerta!

Uvlâkut tuppagangapta, Jêsusib ikkingit tagva

Puigornagit

Kemmergolavut.

2. Avatingita

Puttortauningit, 
Nuname inôtilluta

Innigilavut tamapta, Erdligivlugit

Ômatiptingne.

\%61. Mel. 89.

Gott, ich danke Dir von Herzen.

1. Ômamut kujagivagit Sapputidlalauramga Gûde, nangianartunit Unnuarme kângertome, Sâtanasib kassêtub AкKonalaungimanga.

2. Tessiulaunga Nâlegar ! Arvertartilungalo Okautsitit malliklugit; Sapputilaungmingalo Uvlometauk, assingnik SapputjauveKangimat.

\section{Mel. 184 a.}

Mein Morgensegen sind die Wunden. 1031.

1. Jêsub ikkingit erkarlakka Tuppaleruma uvlâkut, Tamât sullinimne pilakka, Najortigitsainarlugit, Unnugangallo anernipkut Ailaulanga Jêsusima Timingat illijauvianut Najorlugo sinniklunga.

2. Kanoxtôk Jêsusiga tamât Missigênarlago aggut,
Siorniornitit tamaita

Kemergolakka tarnipkut Âniarnut tamainut Gûde Uvlut tamaita pairinga, Uvlâmit unnungmut illumne

Nuname inôtillunga.

96อ. Mel. $151 \mathrm{i}$.

Den Glauben in mir stärke. *1509.

1. Jêsumut okpertinga,

Gûde Atâtaga!

Saimarasuarlunga

Toккоtigingmanga;

Sungertôtigivagit

OKaravit imâk:

Jêsusib pêjarmagit

Ajortut uvamnit.

2. Taimaimattauk, illingnik

Tættekartiminga,

Ama ningarijimnik

Nagliktilaungminga,

Tâpkoa sâtilugit

Nellâgôrnernulle,

Uvaptut nagliklugit

Taimak perкogamga.

3. Jêsuse, Nâlegara

Kujagidlarpagit,

Tunnitsivigigamga

Pijariakartamnik

Attit nakorivara

Pituarigapkit,

Timingnik nerrilanga,

Immingalo aungnik. 
964. Mel. 185.

Piulijivut ikkajortigut

Ullapкoserlutalo

Idluarijarnut sorairata,

Kuviasukovluta.

Kaut tamâlo rajartorangapta,

Sumiglônêt sullijarangapta,

UvloK nâniarlavut Jêsuse najornerne.

965. Mel. 79.

1. Naglingniktotôgavit Tuksiadlarpaptigit, Erkilaungmitigut, Aulingnullo ikkingnut Torkulaungmitiguttôk, Perkonnata ajortomut.

2. Taimak uvlor nâlavut Uvlâmit unnuamut, Aniallo tamât

Erkaumatsainarlugo Allarungnailugolo, Inôtigidlaraptigit.

\section{Mel. 14.}

Hab' Dank, o Jesu! habe Dank. 1033.

1. Nertorlagit Jêsusiga!

Naglingniktôgavit;

KanoktôK inôtillunga Kujagidlarlagit!
2. Jêsusiga uvlometaur Issumagingminga, IdluaringitarnilloSapputilaungminga.

3. Tussalauruk kennunera Idluarilugo, Aularvimne Jêsusiga! Aiparilungalo.

4. Uvlome sulliæksakka Oritilaungmigit, Kuviasutsainarkovlunga Pituarilutit.

76\%. Mel. 184 a.

Im Schatten dess, so ich begehret. 1034.

Piulijiga najorlugo Merngoêrsitsiarpunga Illumnelo missigivlugo Ijimne sikkungivunga; Tuppaklunga makkini- vunga

Tâpsoma najorningane, Missigigapko serrilunga. Hallêlujârvigivara.

\section{Mel. 10.}

Wach' auf, mein Herz, und singe. 1035 .

1. Jêsuse nagliktiga! Tuksiarpagit mânna: 
Uvlome tessiunga

Illingnit pêrKonanga.

2. Anernernut najunga, Ômatimne nippinga TussatsainarlagotốK Sullinimne tamaine.

3. Timimnik tarnimniglo

Tunnitsivigivagit, Tâpkoa sapputikik, Aungnullo ubvarlugik.

ว69. Mel. 244.

Ikkitit Jêsusiga

Issumagigupkit, Illa uvlut tamaita

Inôtigivlugit, Tuppalerangama Sinnilerangama Allaromangilakka Ovanêtillunga.

ร70. Mel. 146.

O Jesu, süsses Licht. 1036.

1. KaumajoK Jêsuse!

Unnuak Kângermingmat Saimarsainît ama Illumne pilerivok, Mânna illûnarma Erkomalerpunga, Ômamut illingnut Kâkpunga nellâgut.

2. Kanorle Gûdiga! Kutsaservigivagit? Saimanernut tagra
Pitinga illûnarma,

Uvlome tarniga

Pairællugo piuk:

Tagva kailerpara,

Tâmna nekkokssiuk!

3. Timigatauk piuk,

Innigitsainarlugo

Perkutigilauruk

Ivsornaitilugolo.

Inôgutiksara

Jêsus! ômartinga!

Tarnît tarnigalo

Attausingortikik.

4. Uvlome Jêsuse

Erkaumatsainarlagit

Illûnânênivit

Illumatsainarmanga;

Piniaræksakka

Gûd nertornautingnut,

Illingakovlugit

Uvlut nungulugit.

\section{Mel. 22.}

Du heilige Dreieinigkeit. $\$ 1517$.

1. Gude, Atâta, Ernerlo Anernek ajunginerlo, Nertornautingnut uvlome Perkovlunga ikkajunga.

2. Pairilaunga sapputinga, Saimarlunga kaumaksanga,

Tækkosanga, illûnarma Ullapirsalaungmingalo. 


\section{ร92. Mel. $151 \mathrm{i}$.}

Aus meines Herzens Grunde. 1038.

1. Illungertornermulle Ômamut, Gûdiga! Kutsaservigivagit Nunamêtillunga, Uvlomelo nutâmik Nertoromavagit Jêsusekut pivagit Nâlegarigapkit.

2. Saimanernut unnuar Pairiluaramga,
Nangianarnermillo Sapputilauramga Nelloprotidlarpunga; Kennuvigivagit: Ajornikka pêjarkit Nâpkigilungalo.

3. Gûde remmangilara,

Kemangimangalo Ôma tessiorpânga Tammalerkonnanga Nagligidlalaukpânga Uvlâmit unungmut Nutakkatut nellâgut Pairitsainarmanga.

\section{IMGERUTINIK PINIARNERMIK.}

ร9อ. Mel. 79.

In Jesu Namen ganz alleine. 1041.

1. Jêsusib attingane

Mânna pigiarpunga

Sulliæksamnigle;

KanoKtôk pilaulanga

Tâpsotunak, tagvale

Sullitsiarniarpunga.

2. Nâlegar ikkajunga!

Piginæk ajorpunga,

Sangêdlarlungalo,

Taimaimat sullinimne

Najornernênartinga,

Issumangnik perkovlunga.

\%94. Mel. 146.

Komm Segen aus der Höh'. 1042.

Jêsuse, takpângat

Saimakit sulinikka!
Anernek timelo

Nekkorsitilaungmikik,

Pigârtilaungminga,

Nâlektilungalo,

Ômat, sennanerme

Illingnêkovlugo.

\section{Mel. 121.}

Nun ich verlasse mich. 1043.

Nâlegak Jêsuse

Tættigigapkille,

Aukpit kibliklinga

Nekkorsilungalo

Erкeasukonanga,

Attaruilinera

Sennajæksamnullo,

Aungnit pivara. 


\section{Imgerutinik piniarnermik.}

996. Mel. 14.

Nun dann, so fange ich mein Werk. 1045 .

1. Atterne sullijæksakka Pigialerpakka, Anernerpit ikkajorlinga NâmakserKovlugit.

2. Unêt sangêtorsôgama Piniarkojatit
Tamât kunnugingilakka Nâjomadlarlugit.

\section{8\%. Mel. 84.}

Unsern Ausgang segne Gott. 1047.

Gûde, saimartilaukit Anninivut, itternivut; Nerkiksavut saimakit, Saimakillo sullinivut, Ikkivit missuksoanga Pitsainarle illuptingnut.

\section{NERRIMÂRSIUTINNIK IMGERUTINIK.}

(a) SIVORNGANE.

988. Mel. 1.

Nâlegan Jêsuse illagittigut

Nerkiksavullo pitsartutilaukit.

999. Mel. 119.

Täglich Brod. 1015.

NerkiksaK :,:

Pijariakartavut,
Kilangmit tikkilauktotit, Tarne perlertuksaungmat Illingnik nerrilaungitor: Tarnima killanârivâtit, Tunninga - illingnik.

\section{Mel. 23.}

Jêsuse! nerrigangapta Toкkut erкatsainarlavut; Jêsuse, immerangapta Ikkingnut kivititigut!

(b) KINGORNGANE.

781. Mel. 1.

Danket dem Herrn, denn. 1058. 1. Tamapse Nâlegak Kujagisiuk!
Illannârimut nerritimmattigut,

2. Tâpsoma naglingninga uvaptingnik 
(b) Kingorngane.

Soraingimat illa, nâkksaungitomut.

3. Gûdib tunnergutingit nerkiksavut,

Pitjutigællugit opigilavut.

4. Nagliktor Nâlegar tuksiarpavut

Nutâmik tunnitsiviojamamut.

\section{Mel. 10.}

1. Gûde kujagilavut Opigidlarlugolo, Tunnergutit pivlugit, Pijavut tâpsomangat.

2. Time, tarne, inôser

Tunnilaukpait nangmineK; Tâpkoa pairilugit Kunnutuitsainarpoк.

\section{INGERGARNERMIK IMGERUTINIK.}

\section{Mel. 22.}

In Gottes Namen wallen wir.

1. Atterne ingergagapta Saimainernik tussuvogut Atâtak! tuksiarapta, Tamainit sapputitigut.

2. Illagittigut Kristuse Tamât apkotiksaptingne, Idluitunit tamainit Piulitigut aggangnut.

3. Gûdiptalo Anerningat PairitsainarlitiguttốK, Nangiarnartomêgupta PitsartutilitiguttôK.
984. Mel. 97.

1. Tamângat aulalerapta, Erkaikattigêtsainarta! Tuksiartigêklutalo, Najortautsainarkovluta Jêsub tokkunganut aunganullo,

Anerningallo tessiorlitigut.

2. Imâgìo tuksiarmita:

Tamâne inôtilluta

Piulijipta ikkingit

Okausingillo ômame

Erkaumadlarlugit sorairata,

Perkojanginigló nâlekluta. 


\section{UNNUKTOME IMGERUTINIK.}

\section{Mel. 164.}

Mit Dir will ich zu Bette gehn. 1066.

1. Jêsuse aiparilutit

Innangajomavunga,

Tamât tættigênarapkit

Pairitsainalaungminga

Nâpkiningnernulle!

Sumik erksinanga;

Najornerne sinnilertor

Serrilune makkinivoк.

2. Takkakka tiglertillugit,

Tarnima errilitit;

Ômallo aulatillugo,

Tusunera imaile,

Taimak sinniklanga:

Jêsus pigivarma, Illa Jêsus pigivagit Avitauniangitomik.

\section{Mel. 141.}

Leg' Dich schlafen nieder, 1072.

1. Jêsub tallinganut

Innangavunga,

Tarniga timiga

Perkolerpâka

Sapputsininganut;

Kênangallotôk

Aungerotilaurtub,

Tækkonârlinga.

2. Sinniktilauktigut Sapputsinerne,
Sinnektômagupta,

Ânianernik,

Tarningêrnerniglo

Tautuktitigut,

Pilorerkovluta sinniktilluta.

3. Sinnilerkârnanga

Erkailerpunga

Taipsomane Jêsus!

Sinnilaunernik.

Umiakotôme

Nangianartome ;

Taimak sinniktinga

Nangiarnanga.

78\%. Mel. 22 a.

Wenn uns're Augen schlafen ein. 1069 .

1. Timivut sinniktillugit,

Umativut erkomalit,

Aggangnut sapputitigut

Perkonnata ajortomut.

2. Engelit tillidlarlugit,

Udsertortaukolerluta, Timivut sinniktillugit Ajortunit sapputikit.

\section{\%88. Mel. 165.}

Wenn mein' Augen schon sich. 1065.

Ijîkka sikkungipannik Merngoêrsertillugik, 
Torârkojominadlarpor

Ômatiga illingnut, Nægliktiga Jêsuse!

Ungagidlarapkille, Illingnêtsainalaulanga Unnuar sinniktillunga.

\section{Mel. 132.}

Stärk' mich mit Deinem Freudengeist. 231.

Najortigut Anernernut, Aungnut inûlitigut Kidjijârnerilauktarnut Serparsalaungmitigut; Jêsusiga tussartigut! Ikkingnut torkormitigut, Sinnitsiarkovluta.

\section{Mel. 75.}

In's Blut, o Jesu, Dein. 469.

1. Aungnut Jêsusiga! Najortigut mânna, Aggangnut puttolingnut Saimausilaungmitigut; Sapputilauktigullo Sinnigomalerapta.

2. Kaulerpat, amalo Tuppalermigupta Sinnigniptingnit, tagva Timingnik ikkilingmik Aunârtorsôjomiglo Tautukôrtimitigut.
891. Mel. 151 i.

Uvlut taimaita tokKut, Anianit aullo Ômatimnêtsainarlit. Kuviagigapkit. Sinnilertillungalo ErKomatillunga Missigilaungmilanga, Ullapirsautingnik.

792. Mel. 68.

Blut'ger Schmerzensmann.

Aulik Jêsuse!

PigittiguttôK Innangajomalerapta, Nâpkigitigut tamapta, Illanârimut,

Piuligaptigut.

\section{ช93. Mel. 79.}

Wenn ich mich schlafen lege. 1075 .

1. Innangalerangama

Erkaimalermigama

Uvamnut pijarnik, Nertorpagit Jêsuse! Nâpkiginga unnuar, Sinniklunga najorlagit.

2. Sinnigomalerama Kaujivat tussunera; Sinnektômalanga Âniarnik pivlunga; Illa sinniktillunga Najorlitit ômatima. 


\section{Mel. 36 .}

Die Nacht ist kommen drin.

$$
\text { * } 1575 \text {. }
$$

1. Unnungme merngoêrserniarupta,

Gûdib idluarijanganut pita!

Saimaninga rebbigitsiarlugo

Naglingningalo.

2. Sinnilerupta erkaumalaptigit,

Tuppalerupta kuviagilaptigit,

Najornerne sinnektômagilavut

Auggit ikkitit.

3. Ômativut aungnut idluarsakit

Piungitut kajunginerinagit,

Erkainiksavut âkikit tokrungnut,

Inûlitigut.

4. Parngnanaersimavingmêtut pivlutit,

Kiksadlartullo, mannigorsâdlakit,

Sorrutsit Kannimajullo

\section{pairikit}

Najornernuttôk.

\section{Mel. 79.}

Wo bist du Sonne blieben. 1083 .

1. Sekкinek nippilermat, Unnuak tikkilerpok, Târsilermat mânna; Taimaimat, Jêsusima Kaumaksarpâ tarniga Kuviasuktilugolo.

2. Uvlok tachesimavok Kilak pinnarnarsivok, Uvloriakarmut.

Mângallo aularuma Pinnarnarsilârpunga Nungupatta idluitut.

3. Timiga tarningêrpat Makkoa annorâkka Attorungnaipakka. Jêsusible tarniga Annorâkartilârpâ Kilaub pinnarnarninganik.

4. Merngortortok timiga Merngoêrserkovara Innangalerlunga, Taimaktauk erkarpara Assia innangaviksak Illuvermêniksarale.

5. Uerngadlarnermulle, Nanênajarkak tagva Timiga tarnelo? Jêsus! nâpkigilugik, Sapputênalaungmigik Okpertut udsertorangne. 
6. Arngnaungub piarkane

Ivavait esarkungminut:

Taimak kibbiktinga!

Pijaukonnago sumut,

Jêsuse! Engelingnut

Sapputitsainalaungminga.
7. Illakkale okorse!

Gûdib sapputilise Nangiarkonnase, Sinnitsiarkovluse, Pairijaukovluselo Engelivaksoanginut.

\section{ENGELINIK.}

796. Mel. $22 \mathrm{e}$.

Herr Gott, Dich loben alle wir. 1088.

1. Nâlegar! nakkorivlutit Kujagênarivaptigit, Engelit pingortinangne Sângnêtsainartut kilangme.

2. Taipkoa kaumajôvlutik Illissimajoksôvlutik

Tækkonâtsainarpât kênat, Nâleklugo Jêsus, nippît.

3. Kikkaratik sinningilat, Piniarningit imaiput: Jêsuse naipertorlutit, Illagêt udsertorlugit.

4. Okpertut sapputênarpait Sâtanas agviarivât, Perkonnagit ajortunut, Uivêriklerninganut.

5. Taimaimat nertorpaptigit Gûde, Kujagiviklutit
Engelinut hailiginut Sapputijaukogaptigut.

6. Inukotitit tamaita Engelinut sapputsaulit, Jêsuse opigingmattit Orautsitit nâleklugit.

\section{Mel. 70.}

Die Gotteshelden. 1091.

1. Pitsartudlartut

Kuvianartomik

Kaujititsijut

InuKotinginik,

Ômisuktullônêt unnuktut Tagvainak pêrlugit perrojauvut.

2. Anerneojut

Ivsornaidlartose!

Pâmangavose

Gûdipse sângane

Opigungnipse angininga Innotsainarpavulle tamaine: 
3. Kattimagapta

Erdlingnadlartose

Illagêkluta

Salutidlarpapse

Kujalimut, sangêtogulle,

Nippeкакârata pâne kilangme.

\section{Mel. 39.}

Wir danken's dem Heiland. 1093.

Jêsus Piulijivut kujagivavut

Engelille kivgartutsainarmattigut;

Tammarkattarluta sangêgalloartogut

Taipkoale ânanaujut ivsornaiput.

\section{Mel. 159.}

Wenn wir in dulci Jubilo. 1094.

Gûb sauganga nâleklugo

Tuksiarangapta,

Nertudlarangaptigolo,

Tussarnertor illa!

Engelit illagiluta

Tuksiarkattigælluta,

Âpaillo serrijutivut

Kilangmiôjunut.

\section{Mel. 249.}

Die Gottes-Seraphim. 1096.

1. Engelivaksuit

Nertordlertoksuit
Kaumainartoksuit

Ivsornaitose, kênase

Mattulugit imgertose:

Hailig, hailig Gûde!

Hailig Gûde,

Nâlegak Jêsuse!

2. Jêsub illagêngita,

Timekattingita

Imgerkattigivait

Kilangmiut tuksiartut

Illagêt Piulijingat

Gûdib Saugârsunga

Tokkotitak

Gûdivut opinak!

3. Tikkipat Nâlegak Ananaujorsoak, Ikkilersimajok,

Okpingitut erksigivât, Okpertut kittiksaungitut Imgervigidlarpât

Nertorlugo

Kappimaningalo.

\section{Mel. 164.}

Ihr Schaaren vor des Lammes Thron. 1097.

Sânganêtose Saugârsub Engelivaksoangit,

Gûdib Erninga kilangmut

Paungautingmigapsiuk:

Angergartoselo,

Time kemmaklugo,

Jêsusemik imgeritse.

Pâne, uvagullo mâne. 


\section{IMGERUTINIK NERTORDLERUTIKSANIK KUJALITIKSANIGLO.}

802. Mel. 61.

L o be den Herrn, den mächtigen. 1100 .

1. Nâlegak nâlengnartoksoak nertulauruktôk Tarnîk! tamanna ômamut tussugidlarpará.

Kailaurittôk,

Kujalidlarlutillo

Sagvairit opigungnernik.

2. Nâlegar nertoruk mânna, pitsartutingmattit, Piksakartilutillo kilangmit naglingnermik;

Erkariuk, Ajugakangitôjub Issumagitsainarmattit.

\section{Mel. 132 d.}

Sei Lob und Ehr dem höchsten Gut. 1101.

*1. Gûde nakoridlarlavut Atâta saimarsaijox, Tattamnartullitsainartok, Gûd ullapirsartivut! Mannigutsainarmattigut, Pêjarmagit kiksarnivut; Gûdivut nertorlavut!

*2. Pakkoa nakoridlarpât Engelit pitsartujut,
Mâne kujagidlarivât Okpertut nunamêtut; Tamapta nakorilavut! Anerniggillo inôjut Attinga nertorlitsuk!

3. Gûdiga inôtillunga Kutsaservigivagit; Nertornermik, Nâlegara! Illûnatik pivâtit; Kuviasugit ômatîk Anernerma timimalo Gûd nakoridlarlikko!

4. Nâlegar avitsængilar Illagêktitaminit; Tâpkoninga saimarsaijub, Ullapkositsainarpait, Anânatut kittorngane Tessiorpait aggangminut: Gûd nakoridlarsiuk!

5. Kujagijartorlugolo Tikkisiuk tamapse Nertoriartorlugolo Imâk tuksiaritse: Tamaita issumagivait Tamaita idluarsarpait; Gûd nakoridlarsiuk!

\section{Mel. 168.}

Lasst uns unsern König loben. *1604.

Nâlegavut nertorlavut! Kinguvâkattigênut 
Opigijaule illûnâne;

NunaKattigêksuit

Sânganut sêrkortulaulit,

Ômame kibliktaulutik,

Sorlo kilangmiunut

Nakorijautsainarmat.

\section{Mel. 146.}

Nun danket alle Gott. 1003.

1. Gûd nakorilavut

Ômamut illûnânut,

Tattamnadlartomik

Uvaptingnut ping malle;

Inûlerviptingnit

Mânnamullo aglât,

Serngnigivâtigut

Illa uvlut tamât.

2. Gûde tussartigut

Kennuvigigaptigit:

Opigosuktunik

ÔmateKartitigut.

Uvlullo tamaita

Piloriktitigut

Pitit illûnaita

Pitsartutilugit.

\section{Mel. 225.}

Singt dem Herrn. 1110.

1. Nâlegak nertornak! Tamapse nertorsiuk! Pitokar itsungnaimat
Nutaunerlo pimangmat.

Nutânik issumaggit

Imgerlutiglo pilit:

Nertornautâ attorlugo,

Illûnatik pitsainarlit

Illûnâne inôjut!

2. Gûdivut Jehôva

Imgerutigisiuk;

Taimaitsungnartoselo,

Aunga âniangillo

Inungnut illûnainut

Sorairase taisigik.

Nellojullo illûnaita

Angijomik Kaikungmagit,

Nellojungnaitisigik.

3. Aititse, nellojut

Nunanginut, inungit

Okautisigik Gûdib

Tamainut nâlegaujub

Nuname nâlegauvik

Âkingmago ingminut.

Nellojose sâgiaritse

Nâlekamut, okpingitut

Erkartoromârmagit.

4. Jêsusib Kristusib

Gûdib annerijangat,

Inuit illûnaita

Sakkertitsomârmagit,

Idluartotuamik

Erkartorniarlugit.

Okpertulle illûnaita

Ittertipait iglominut

Hosianamik pita! 
80\%. Mel. 14.

Er hat noch niemals was verseh'n. 1106.

1. Jêsus aulatsinermine Tammarungnangilak, Piniarningit illûnatik Nertornatsainarput.

2. Taimaimat tættigileruk Nunamêtillutit

Ôma nâmatsainartomik Pairitsiarmattit.

3. Illuptigut keagupta Nâpkigivaptigut, Gûdivut tunuergutingnut Serritipaptigut.

4. Okpertut keavangningit Kittipattit tamât, Mikkinerpâk kugvinginit Erkaumavat aglât.

5. Nuname inôtilluta Inôtigivavut, Angergarsimaguptalo Pigidlalârparut.

\section{Mel. 227.}

Lasset uxs den Herren preisen. 1113.

1. Nâlegar nakorilavut Nertornauta :; perorle Perkutaujose ômunga Nertutsainad :,: larsiuk! Nâpkigitsainarmattigut,
Erkitsomangmattigullo, Naglimut uvaptingnik Pêjarpait ajornivut, OKaritse attinganik, Illa annerimajose, Piniarningit tamaita Nertornautik :,: sarællugit.

2. Inûlerkârtinnatale, Illitari :,: vâtigut, Annerilaukpâtigullo Jêsusekut, Jêsuse Kristusekut.

Aglât killaglo nunalo Kivgaridlarlugillotauk, Naglimut Ernermigut Kittorngaringmattigut, Saimaninga soraingimat, Piksarsitsainarpogulle; Taimaimat pinasuarta Tamainille, tamainit ungagilugo.

3. Mânna Kuviasungnermut Atâtapting :,: uut pita! Najorninganêtutitut Opigomut :,: imgerta : Hailig, hailig, hailig, illa Nertorsiuk, Hallêluja! Nagliklugo Gûdivut, Illa Piulijivut

Nertordlerutigilavut Naglingninga uvaptingnik, Aglât inukotiminut Anneridla :;: laungmattigut. 
4. Aungmut pissiarilaungmase

Illitarid :,: larsiuk,

Kattangutise Jêsuse

Tættigilu :,: arsiuk ;

Illannârijigilugo,

Piulijigidlarlugo

Kattangutigisiuk,

Kuviasutigisiuk;

Piloriktitaudlarpose,

Ôma piulilaungmase;

Erkatsainarlugo mânna

Nertorlavut :,: illûnata.

*. Iksivautamut aititse

Saimarvigi :,: jauvingmut!

Nâpkigijautuinarapse

Gûdipta er :,: ninganut.

Killanâdlarporlo tâmna

Erkitsomavluta illa

Perkumik angnerpâmik Aungminut pillidluta. Saimanadlarmêk illâle!

Ingminik aitortudlarmat, Ômalo naglingninganit Avitaujung :,: nanginapta.

6. Perkangarviksax tâpsoma Uvaptingnut :,: angmarpâ, Illungertorlune mânna Kaikovluta :,: ingminut, Tagvangat nutakkatitut Saimanermik perkovluta Kâktomik ômatelik, Pekartipâ piminik. Nungusuitôjor tâmna Attutsainarlavut : tagva
Piloriktilârpâtigut Issokangi :,: toksoarmut.

\section{Mel. 195.}

Gott ist gegenwärtig. 1117.

1. Gûd uvaptingnêpor Kenuvigilavut Opigukta sajungnermut Akkonaptingnêmat Nekkorsitiluta, Nellopkotimut pâmakta! Tâpsoma tamapta Kennilaungmattigut, Nakoridlarlavut!

2. Gûd uvaptingnêpor, Tâmnalo Serafit Kivgartorpât sorairatik; Hailig, hailig, hailig! Engelit imgerput Tamât nertornautinganut: Taimaktauk nippivut, Gûd! tussalaungmigit, Kujagigaptigit.

3. Gûdeviksôjotit, Kanortôx uvagut Nertorlaptigit ômamut; Sorlo Engelivit Naipertudlarmattit Kênarniglo tækkovaktut; Taimak Gûd tamainut Idluarijarnut Inôtimitigut! 
$314 \quad$ Imgerutinik nertordlerutiksanik

4. Issumatunata

Gûd taimaititigut

Ullapirsalaungmitigut.

Sorlo perorsiat

Sekkinijârnermut

Perorsitsiartutitut;

Illume Jêsuse

Perortimitigut!

Napkigosungnernut.

5. Nâlegan! illuvut

Innigilaungmigit,

Nuname ivsornaitikit;

Kailaurit Jêsuse,

Kaumaksarmitigut

Nâleklutit naglingnermut,

Ômamut illingnut

Kêmitênarluta

Illingnêkovluta.

\section{Mel. 230.}

Auf, auf, mein Geist. *1624.

1. Attê annernîk tarnîklo, Atte timîk, issumakkalo, Attê ômattîk augalo, Attê pitsartunîk tamât! Kuviasugitse Jêsume. Nakorilugo pititse, Imgervigisiuk Kujagilugolo! Sakrêtitse opigungmik, Serrinermik Ânianingit pivlugit!

2. Illa ikkitit pivlugit Jêsuse illûnarma mâne
Nakoritsainadlarlitit!

Tagva uvlumne tamaine, ToKkovit kiblêninga illa;

Illumne missigivara;

Ijîkka torârlik

'Tokkotaudlarvingnut!

Siutîkka - tussarkôrlik

Okautsinik

Pjvluta ânianernik!

\section{Mel. 249.}

Du Herr bist unser Licht. 681 .

Kaumartigivaptigit;

Kittorngarijatit

Kematsangilattit,

Kaumanerne okpernerme,

Arvertarpogullo sângne,

Kuviasukluta,

Nâpkiningnît

Nutângotsainarmat.

\section{Mel. 90.}

Geht, werft euch vor die Majestät. 1118.

1. Gûb nagligosuktotôjub Sângane pâmagitse; Saimarsaijok najorsiuk, Nâlegar opinartor! Tautunggalloarpasse, Mâne okpervigijase.

2. Jêsuse inûliklerte! Tarninik illûnainik! Kannimajut tikkititit 
Nuname âkigangne;

Uvangatauk aktorapkit Aungnut inûlilaungminga.

3. Jêsuse inukotingnut Nellâgut kittinamga, Nertorpagit; ômatingnut Kellaksulaungmigamga; Atâta tuksiarangne Pivlungatauk pigaville.

4. Tagga illa, ômatiga Aniajutigigangne, Illingnut tunnilerpara Inûlijaukovlugo, Âniarnut ikkingnullo Ikkomatiniarlugo.

5. Kivgartorlutit Gûdiga! Angerpunga ômamut, Illûnarma âkingminga Timipkut tarnipkullo, Kaujilit kaujijomajut Inukutaugama Gûmut.

\section{Mel. 58.}

Gottlob! dass unser Herr. *1631.

1. Nâlegar Jêsus nertorlavut (Inuartuk akkorngangnele Kikkiæktortaujoк)

Aniadlartok,

Ômatiptalo kaujimangmatsuk

Gûdeongmat.
2. Opigijaule miksekârneк, Angnerpâkutiginiarpât Illa ômatipta, Jêsub sânganut Sêrkut sêrKortortuksaudlarmatta

Illûnatik.

3. Taimaimat, issokangitomut

Mâne pânelo imaitsomârmat:

Nuname kejungme,

Nivingalauktok,

Illûnainut nertortaujuksaungmat

Gûdeongmat.

\section{Mel. 206.}

Herr Zebaot, Du wahrer Gott. 1119.

1. Kilangmullo, nunamullo

Nâlegaujor! Gûdeojor!

Pingortit :,: sigavit

Gûd sillaksoarmilk

Kissivit pairksijô - votit Ajortunut kattalauktut Aungnut pissiarigangne, Idluarsarlugit Opigivaptigit.

2. Kernbillo Serafillo Sajuklutik nâlekpâtit Jehôva :,: ogavit; Kristuseojotit :,: igvit! Aut kovijok erdlingnartor 
Pattangajut nunamêtut, Nungusuitomut Inôtigidlarpât.

\section{4a, Mel. 208 d.}

O ihr Gottesheere. 1122.

1. Piulimajose,

Imgitsiaritse

Kujalinermik!

Illûnamarita

Illa nertordlerta

Gûb sauganganik!

Tamapta ingergapta

Asserorsimadlartogut

Kellujaujogulle.

2. Nertordlerutivut

Kugviornivullo

Nachogingilait

Kujalijutinik

Serrijutiniglo

Pijungnangitok,

Taimaitor kiksarlune

Jêsusemut aijuksauvok, Kugvit Kaujingmagit.

3. Naglingniojotit,

Pitit tækkonâkit!

Uvagut tagga!

Ômativut pikit,

Ônarsitilaukit

Nutâmik mânna!

Sangêtut saimanernut

Kippalôjungnaermariglit Ikkitit pivlugit!
4. Kivgatit tamaita

Pairitsialaita

Annorâmiktôk

Ubvartaumajomik

Aungnut ânanâmik

Pillilaukittauk!

Sullititsialaukit,

Kuviasungnermut Kugvingit

Ablatsautilugit!

5. Illa kittorngatit,

Piniutijatit

Piulijaunermik,

Pairigannilaukit!

Nertorniarpâtit

Tagva illûnatik;

Mânêtut sangêtokut

Pânêtulle nâmaktomik

Tussarnidlartomik.

\section{4t. Mel. 595 a.}

To God the only wise. 687 .

1. Sillatujotuak

Gûde attanivut

Nertortaule, itsaksoak

Piulingmattigut.

2. Aulatsiningata

Pairidlarpâtigut

Ajortogut sulle mânna

Issokangitomut

3. Jêsusib tarnivut Sapkuniangilait, 


\section{кujalitiksaniglo.}

Tamaita Atâtaminut

Pititsomadlarpait.

4. Kattijomârpatta

Piulijaumajut

Takpâne, Kanoktôk tagva

Illauta uvagut!

815. Mel. 164.

Gelobt sei Jesus, Gottes Lamm. 1124.

Jêsuse Gûb Saugârsunga

Opigidlarlavuttôk!

Nakorilugo, ômunga

Piulijaumagapta.

Nâpkigingmattigut

Nertutsainarlavut!

Nunamiut rilangmiut

Anerniggit nertorlitsuk!
816. Mel. 39.

$\mathrm{O}$ dass Ihn doch jedes. 1125 .

1. Attêtôk! illûnamassiata mânna,

Jêsus nagligilavut inôtilluta!

Tarniptigut kuviasugvigilugo

Anerniptigullo nakoridlarlugo.

2. Saugârsuk, tâmna, tarniptingnik tamainik, Aungminut kovimajomut pissilauktor,

Illanâk naglimut tokkotigijivut,

Illûnata illa nakoridlarlavut!

\section{KRISTUSIB TIKKITSOMÂRNINGANIK ÂNANAUNER- MINE, ÔMINGALO NERRIUNGNIPTINGNIK.}

\section{1\%. Mel. 1321.}

Es ist gewisslich bald die Zeit. 1126.

1. Nelliutilârpor illa

Jêsusib akKarvia,

Erkartorniarpait tagva

Inuit illûnaita;

Kuksanarpok, silla tamât Ikkomamut nungutaukpat, Sorlo Pêtrus aglangmat.

2. Tiptulausijaromârput Engelit illûnâne,
ToKkungajut ômarlutik Tagvainak makkilârput; Taimaipat sulle inôjut, Tagva tagvainak Gûdemut Ablatsângortitauvut.

3. Jêsusiga! taimailerpat Ikkingnut piulinga, Annerilauktavit pâne Akkornganêkovlunga, Illakka illagællugit Kilangmut ittertiminga, Tokkotigilauramga. 


\section{Mel. 211.}

Liebster Jesu, liebstes Lieben. 1127.

Nagliktara Jêsusiga, Gûdiblo Saugârsunga, Pêjaijor ajornimnik!

Pituarijomavagit

Torkungnut piuligamga

Ungagituarivagit;

Igvit naglerkâramga

Piulidlarlungalo,

Ô-ma-mut nagligijomadlarpagit.

Piulijauvut tâpkoa :,: Nerrikattaujuksat Saugârsûb Komuniôninganik :,:

\section{Mel. 16.}

Wol dem, welcher seine Londen. 1128.

1. Pilorikpok inuk tâmna, Opallungaitsainartor, Kaumajome arvertartoK, Sokкоsijuilune.

2. Pilorikpor kollerminik Orksêtsainasuartor UiksaK mullulerкôrpat; Tâmna sillatojôvor.

3. Kittorngavit pairijingat Sinnijuitôjotit: Sâlakartungortiminga Uerngatuaruma.
4. Issumakka tuppaktikit, Pigâtsainarkovlunga, Illingne sinnisitinga, Kaikonialerumga.

\section{Mel. 161.}

O ihr auserwählten Kinder. 1129.

1. Okpertut Kennersimajut, Jêsusib kittorngangit Erkeasuktailititse!

Kina akkonapsingne Nippungajor Erkeasuktor?

Pigârtuksautsainarnise Kamagijomangilase?

2. Pigâritse! pissiluse Orksuksapsingnik illa, Kaumakovlugit illuse Ketterarmat unnuak; Gûb Erninga Kaingmat illa, Angijomik kaikungmase Sânganut aijuksauvose.

3. Taimaipat time tarnelo Inulijaudlalerlîk ! Ullapirsartaukovlunga Kaumalunga nellâgut. NerriungneK Nellautsile! Kuvianarninganullo Itterungnadlarkovlunga.

4. Tussarnertokaromârpok Kilangme imgertunik, 
Engelit imgerkattauvut

Serritigijamingnik;

Piulimajut

Illûnatik

Harfesijarkattaulârput

Saugârsuk Gûd nertorlugo.

5. Tussarkôritse okpertut?

Harfesijatsiartunili?

Pâmakterpullo Saugârsub

Iksivautaksoanganut;

Kattangutit

Gûb inungit

Ittigganginut pâmakput

Opigomut Kugviortut.

6. Illagêt serridlaritse Jêsub inukotingit, Kuviasugomârapse Akkorngænne Serafit, Serridlartut

Pânêmiut;

Attoromârnipsingnulle Mâne âkiktauleritse!

\section{Mel. 71.}

O Herr der Herrlichkeit. 1130.

1. Nâlegaviksoak!

Opallungairtinga,

Killanârlunga

Tikkiniarnernik

Nerriunartomik,

Opiguktinga.
2. Immanuêliga

Sapputiuk tarniga

Nippunganermit

Piuliklernivit

Kailauktanganigle,

Pekarkovlunga.

3. Uitilaungminga

Inôlerkovlunga

Kaumajomele;

Jêsus Anernernut

Tessiortauvlunga

Arvertarlanga!

\section{Mel. 75.}

Ach Jesu. wie so schön. 1132 .

1. Nertornartok illa

Illingnêleruma :

Jêsus tækkonâramga

Ullapirsalerparma,

Nunamillo kilangmut

Tikkikuma illingnut.

2. Ijik kanningalo,

Timinga ikkilik

Tækkojomârpavulle,

Tættigidlaraptigik,

Tamaitalo ikkingit

Kemergoniarlugit.

\section{Mel. 230.}

Wachet auf, ruft uns die Stimme. 1133.

1. Pigârtut kotsiktomêtut

Pângat nipliutivâtigut: 
Tuppagitse okpertose! Unnuarlo ketterarmat, Imâk " kaigarsudlarpâtigut:

„Kaititse uigasuit,

„Uiksak kailermat

"Kollisse âkilit,

"Makkiluse upagitse,

„Nerrimavingmut,

„Pagreartolerisiuk!

2. Nipjausijartut Engelit Illagêt tussalerunigit, Tagva kuviasungnermut Illumingne ômatingit Aulavlutik makkisâlerput Nagligijingat kailermat Ânanâksôvlune, Tuksiarvigivât: Gûb Erninga! najugarnut Ittertigut, Najuênalaungmitigut.

\section{Mel. $151 \mathrm{~g}$.}

Macht eure Lampen fertig. 1134.

Kollisse âKisigik,

Orksukartilugit;

Piulijauviksase

Nelliutidlarmat:

Timise tarnniselo

Pijarêrtisigik,

Nipliutijaugapse:

Uiksan kailerpok.
82อ๊. Mel. 79.

Was denkt ihr, wie man's nähme. 984 .

Kanok issumavisê? Nâlegak tikkilerpat Erksinajarkitâ? Aukagle ittiggangit Kugvinut kunniklugit Pâmaktervigidlarpavut.

\section{Mel. 58.}

Weicht alle von mir. 1136.

1. „Kammaksinga idluitose!“ OKautijomârpait tâpkoa, Jêsuse sâlugo Mâne pingitut; Piulijaunatik okpernermut Tokrungavut.

2. Angijomik kuviasukput Illagêt piulijaumajut, Uvloksôjomầrtome Jêsub pissiangit, Tallerpianut pititaugamik, Serrivingmut.

\section{2\%. Mel. 146.}

Wie wär's, wenn der Gesang. 1137.

1. Tikkikunittigut Nagliktub Nâlegapta,

Naipilerpâtigut

Kiksarkattartilluta

Piunginiptingnik; 
ânanaunermine, ômingalo nerriungniptingnik. 321

Ômatiptingnele

Killanârivlugo

Ungagilugolo.

2. Tagva serrivogut,

Koaksângaviginæglo,

Jêsus tikkikuvit

Uvaptingnut nunamut,

Aggavut illingnut,

Ungagijaptingnut

Issangniarpavut,

Illerasugata.

3. Angergartitigut

Ullapirsartauvluta,

Tikkilermiguvit

Erkartuiviksarnut,

Uvagut adsingnut

Makkitilauktigut,

Nangertilutalo

Tallerpingnêtune.

\section{Mel. 185.}

1. Jêsuse pituarigaptigit, Kennuvigivaptigit:

Ômativut innigilaungmigit,

Aungnullo serparsakit;

Idluarijarnut âkikluta

Tikkivingne pijarêrkovluta
Itterкattigivlutit

Kuvianartovingmut.

2. Opallungairsimatimitigut, Jêsuse tikkikuvit,

Sillatojotun-itsainarluta,

Uttakritsainarlutit;

Ikkivit aunganut tokkungnullo

Kiblitsainarlugit ômativut,

Pagrearungnarlutit

Illerasulugata.

\section{Mel. 164.}

1. Okautaujungnatsæengilak Kuviasugviksavut, Tussarupta Gûd okarmat: "Atatab nagliktangit, "Mânna nâlegauvik "Kingormgutisiuk, „Pijarêrtok illipsingnut "Sillab pigiarninganit.“

2. Erksinartôjok imâgle, Okpingitut pikpagit: „Idluitoâlojose "Aularitse uvamnit, "Ikkomarsoarmut "Kamisuitomut, "Pijarêrtok Sâtanemut, „Engelerotinginullo.“ 
322 Anerniptigut arvertarniptingnik xilangme,

\section{ANERNIPTIGUT ARVERTARNIPTINGNIK KILANG- ME, KRISTUSEMÊTSOMAVLUTALO KILLANÂR- NERMIK.}

830. Mel. 106.

Mein Herz lebt schon im Him mel. 1139.

1. Takpâne rilangmêtsarêrpor

Ômatiga, tagvanêmat Perkutiga pikominartok, Jêsusima torkortanga Uvamnut; tagvane illa ToкKoviksakadlarpunga.

2. Illingnut inôjomavunga Perkutigigamga igvit; Tоккоnек piksakautigiva$\mathrm{ra}$,

Inukutigigamgale,

Inônimne tokkumnelo Perkutigitsainarlagit.

3. InôKojomagumga sulle Angituinalerpunga:

Najornernut Kaikolerumga Ku viagiluarpara.

Pijomajarnik nâlektor Kilangmut pijuksaulerpor.

\section{0 a. Mel. 184 a.}

Es halten eitele Gemüter. 1141.

1. Sunaungitunik ivlersijut Nuna pitônasugivât;
Illitarksijulle Jêsumik

Perkutiniglo pânêtunik,

Tâpkoa nunamêgalloar-

Ômatingille pânêput; Arvertarasuarput taimak, Issumat pânêKovlugit.

2. Tagva Paulus avitsomavoK,

Abraha tokкојоmavoк, Hiobe âniangordlune Killanâdlarpor Gûdemut, Eliasib nunamêdlune Tarnine angergautjaukovâ,

Simeon uvlâmit unnungmut

Kaikojâunermik tussuvoK.

3. Nâlekab aulatsijotôjub Inôtsemik toKKomiglo, Pine kippitsungnaertiniarpait

Kaikogamigit piviksame. Angergarnerub Jêsusemut. Soraertipait oKumaitut, Jêsublo Palmit aggangminut

Kaipait sâlakalauktomut. 
Kristusemêtsomavlutalo killanârnermik.

\section{Mel. 83.}

Mich verlangt Dich mit. 1143.

1. Kajungidlalerpunga Jêsus tæklkojomavlugo ; Taimaktauk kilangmiut Nangiarungnaimariktut Illagêniarlugit

Nertoromadlarpara.

2. Allarniarnangatôk! Seningnêtitsainarminga; Tættituarivagit,

Aggangnut tessiulaunga, Kaikoniarkârnanga Illingnut Jêsusiga.

\section{Mel. 75.}

Im Himmel ist mein Freund. *1666.

1. Kilangmêpor illa Illanârijiga

Tunnidlartok uvamnut Ingminik naglingnermut, Piksaerutsængilanga Ôminga pekaruma.

2. Igvit perkutiga Maksoatiparma: Piksariniartavut Illingne, angijôvut, Ânanâvaksoarnut Serritilârpaptigut.

\section{3ือ. Mel. 136.}

Erwünschte Zeit. 1144.

Pijominak!

Kakugo piviksauvâ,
Annerijaumajut illagællugit

Piulijiga tækkojomârapko?

Parngnainasuarpunga, nellonarmat

Uvlomelônêt naipitsungnangimangâpko.

Kaile, serrinartotuak! Pijominak!

\section{3 ณ. Mel. 218.}

Es ist noch eine Ruh' vorhanden. 1145.

Sulle merngoerservekarpoK

Merngortortôjotit, attê! Anersaumijotit tamâne, Siornioravit sulle; Tautukôlauruk takpânêtor

Saugârsuk mianersijôjok, Nangmagæksatit egikit! Sorsungnek mâne nâsaraipor,

Tuavi merngoerservingnut Paunga itterniarpotit.

\section{W. Mel. 230.}

Heil'ger Bräut'gam unsrer Seelen, *277. Anh.

Tarnipta annerijangat Kuvê, pingitoKatsænnata Tamapta illingnêkupta! 
324 Anerniptigut arvertarniptingnik kilangme etc.

Atsuilijut akkorngænne, Ikkingnut inûlimajunut Kuviasukattaugupta! Tagva uvaguttauk Pitinajarluta Atâtavît

Perkutinginut; Tautukpavut Mâne issumanangitut.

\section{3:4. Mel. 244.}

Ich bleibe nahe stehen. $* 1678$.

1. Nâlegak ânialik

Najoromagapkit

Kemergoluarlagit

Kuviagigapkit,

Ittikkatit mânna

Erkiniarlakka,

Ijikik illutortuk

Ullernaigilakka.

2. Annerterigarnerpît, Annersârnerpîllo, Aungmik kiægungnivît Kugviornivîllo, Aiparilaulinga Angergaleruma ; Kuvianartovingmut Illaliorminga.
3. Kaikogumga illingnut

Najuênarlutit,

Nellopkotidlarlunga

Kujagidlarpagit,

Mariatut tagva

Illingnut kaivunga

Ikkitit kunniklugit,

Kemmergolaitalo.

4. Kattangutigigapkit,

Jêsus nagliktiga, Tikkitsomârviksalle Sulle nellovara. Sorlo tautungmattit Illiniartivit,

Taimaktauk kaut tamâttôK

Najutsainarlagit.

\section{8อือ๊. Mel. 37.}

Hier bleibt indess mein Loos. 1150.

Jêsus malliklugo

Piniarpunga,

Nâmagijanganut

Illingavlunga

Taimailiorlunga

Uttakkivara,

Illaukârtinnanga

Takpanêtunut. 


\section{NÂLEKAMUT ANGERGARNERMIK, TIMIBLO MAK- KITSOMÂRNINGANIK.}

836. Mel. 132.

Ein Würmlein bin ich arm und klein. 1159.

1. Koppergotuinauvunga, Illumajaulungalo

Tokkomut erksinartunullo,

Mannigortigivagit:

Igvit Nâlegar. Kristuse

Nangmineк aklulauktotit, Illingnut nippilanga!

2. Tagva aggangnut tarniga Perkojomadlarpara, Gûdik uvamnetsainarit Anernîllo pêrnago;

OKarungnarungnairuma Pikit annersauminikka, Tussarlugillo. Amen.

\section{3\%. Mel. 132.}

Wenn mein Stündlein vorhanden ist. 1162.

1. Piviksara tikkilerpat, Angergarniaruma, Jêsuse aiparilaunga, Ikkajortailinanga, Nâkpat mâne inôsera, Tarniga tunnilârpara Aggangnut, Jêsusiga.
2. Tokromit makkilauravit, Ômartitsomârparma; Токко erksigingilara Tættituarigapkit, Illingnullo Kaileruma Najuênarlutit tagva Kuviasuktiparma.

3. Mânna avatigigamga Mannigorvigivagit, Avitauniangilanga Illingnittauk tokкome; Tokrungnulle inôtsemik Illa nungusuitomik Piksakartilaukparma.

\section{Mel. 83.}

Jesus meine Zuversicht. 1164.

1. Jêsus tættigijara Kellujigalo ômarpoк, Tamanna Kaujigapko, Sattômajuksaungilangâ ? ToкKub illusingata Koaksârtigalloarmanga.

2. Ômarmat Jesusiga Ômadlaromârivunga, Najordlugolo tâmna, Sôg tagva koaksârtuksauvîk.

Avatigigaminga Allaromangilânga. 
3. Allaromangimanga,

KuviasuktoK tarniga,

Tættituarigapko;

Tokkolerpallo timiga,

Mannigortigivara, Adsigijomârapko.

4. Kellaksorsimavunga Okpernermut tâpso-

$$
\text { munga, }
$$

Avisârungnairuma

SakKertitsomârpângale,

Toккub avitilunga

Jêsumit, sapperpânga.

5. Ijîmnut ukkungnunga

Illitarijomârpara

Jêsus Piulijiga;

Ôma serritilârmanga,

Ajulernera tagva

Ikpigijungnaipara.

6. Mâne kugviorpaktut

Pâne pinnarnaromârput,

Mâne ivjungolertut

Ânanâmik makkilârput:

Okumaitullo tagva

Tamât pêjarpait ôma.

7. Timiga mannigorit,

Unêt tokкojuksaujotit, Jêsub timigingmattit Ômartitsomârivâtit, Tussartaukpat nippinga Illuverne tamaine.

8. Uvingub issumangit Sâlagijæksarivasse,
Tarnise tunnilugit

Najoromadlartapsingnut;

Ômamut najorsiuk

Killanârvigijase.

9. Jêsus Piulijiga

Puigorungnailerpara

Nuname inôvlunga,

Aungminut pissiaringmanga

Kujagijomavara

Inôsimne illûnâne.

\section{Mel. 149.}

Schickt das Herze da hinein. 1165.

1.Ômamut najorsiuk,

Jêsusib najugâ,

Kajungervigisiuk,

Najuênarlugo ;

Kaumajok pânêtor Allarungnairsiuk,

KeajungnaiviksaK.

2. Gûd ikkilersimajor

Pivluta toккотак

Tagva ullernaigivât

Kujagidlarlugo.

Mânelo pitôvor

Tokкotar pivluta,

Angerarkârata.

\section{Mel. 132.}

Du gingst in Tod zu unserm Heil. 1166.

Piulivluta toккомок

Pivluta illijauvok, 
Taimaitokuttauk timivut Adsigijomavlugit; Sorlo timâ ikkilertak Ananâmik makkimingmat, Angerviovoguttaur.

\section{Mel. 151 a.}

In meines Herzens Grunde. 1167.

1. Ômatimne atternik Tokkungnik aungniglo

Issumatsainarlanga,

Kuviasukovlunga;

Torkulauruk tarniga

Kikkiaktornernut, Aungnullo ômatiga

Missuktitsainaruk.

2. Jêsusiga tarniga Serngnigilaungmiuk Ajortunit tamainit Sapputilaungmiuk; Illingnêtsainaruma Nunamêtillunga, Kuvianartovingmut Aijomârparmatauk.

3. Nagligijomavagit Ôvanêtillunga, Illûnarma illingnut Tunnijomavunga, Tarniga innigiuk Pissiarigangne, umatigalo piuk Idluarsilugo.
842. Mel. 22.

O Jesu Christ, mein's Lebens Licht. 1168.

1. Nâlegara Jêsuse Krist Sungertûtigênarpagit, Tujormijotun - inama Nunameinôtillunga.

2. Tamanemiôtillunga Ingergajotun - ipunga, Takpaunga angergarvimnut

Tokkungnut pijarêrtomut.

3. Ikkingnut aupalluktanut

- Kidsijârnernullo aungmik Ajortunit ubvarminga Nekkorsitilaungmingalo.

4. Mittautigijaudlarnernut Niakôsertaunernullo, Pêrtaulit pitlautiksakka Idluarsitilungalo.

5. Immerungmik nipleravit Sungalingmik immitaunit Nekkorsijutigilago Kappiasuleranganna.

6. Kappiasumut nippivit A ungmiglo kovilaungnivit Âniavingmit tarniga Piulilikko Jêsuse.

7. Sennerkab kappininganut Tarniga torkulaungmiuk, 
Kemalerpago timiga Illingnêtsainarkovlugo.

8. Engelit illagivlugit Nakorênaromârpagit, Okpertut illagællugit Ijîmnut tækkolârpagit.

\section{Mel. 79.}

Wenn kleine Himmelserben. 1161.

1. Tagva nutarârsuit

Gûmut angergarunik, Assiotsangilat:

Atâtab kilangmêtub Torkotuinarpaille Piulijaudlarkovlugit.

2. Baptijutikut Gûmut Tunnijaudlarmattale, Kittorngarilerpait : Kanorle tagva tokkub AkKonarungnarpagît? Jêsub angergautimmagit.

3. Nuname sorrutsine Perorsaraidlarmatta Ajornerit illa, Taimaimat, Nâlegapta Issumanganut tagva Tunnivavut najugânut.

4. Illa sorrusek igvit! Kuvianartovingmut Aisâdlaleravit, Kuviagivaptigit,
Gûdib kittornganginut Kilangme illauleravit.

\section{Mel. 106.}

Wer weiss, wie nahe mir mein Ende. 1170.

1. Nellonarpok, кanga timiga

Tokкomut pijuksaumangât,

Kingorlerpâk kappianartor

Kaisâdlarniangimangât. Aut pivlugo Kennuvunga Illingnut angergarlanga.

2. Jêsumit avitingilânga Inôtsima tokkublônêt, Tômasetut oxautivara: Gûdigalo Nâlegara! Aut pivlugo kennuvunga: Illingnut angergarlanga.

3. Piulijiga attilaukpara Taimangat baptitaugama, Taimaimat Gûdib naglikpânga

Kittorngarileraminga, Taimaimallo kennuvunga: Aut pivlugo itterlanga.

4. Jêsub timinga nerrivlugo .

Aungalo immilaurapko Puigorungnatsængilânga ; 


\section{timiblo makkitsomârninganik.}

Illumiu tarivara.

Timinga aungalo illa

Pituaritsainarpakka.

\section{Mel. 175.}

Wen hab' ich, Herr, als Dich. 1171.

1. Kina tættigilârpigo Kappiaktoadlarnima Kingorlerpângane nuname?

Kina tarnimnik pairksiva

- Uvanga sangêtôjunga

TokKomut tikkitaulâruma?

Tagva tungavigivagit

Gûdiga Piulijiga.

2. Okpervigimarikpagit

Perkutiginiarlunga

Ikkingne torkorniaramga;

Tagvane erksingilanga,

Sumullônêt okpernera

Sâlagijaujungnatsængimat.

Inôtsimne pigigamga Tokkumne pigigivarma.

\section{Mel. 165.}

Freu' dich sehr, o meine Seele. 1174.

1. Kuviasulerit tarnîk!

Puigolerlutillo

Siarklernernik tamainik
Nâlekab Kaikungmattit:

Mânna aijuksauvotit

Kiksarvigilauktarnit

Kuvianadlartovingmut

Sorainiatsængitomut.

2. Senningnut aijomavunga

Jêsus tokkoleruma;

Ikkikut ittilârpunga

Kilangmut torârvimnut, Illa Paradîsemut

Ajortub aivianut, Jêsus ittertilârparma Pinnarnarsitilungalo.

\section{4\%. Mel. 208.}

Sel'ge Lebens-Stunden. 1180.

Inôviksatuak

Jêsub ikkingine

Mâne nuname!

Tussarnertotuak,

Nakoriguptigo

Jêsub tokкunga! Ânanaunerpâmigle

Pâne inûlijaumajut

Nertudlaromârpât

\section{Mel. 218.}

Nunamik kemailâromajub.

Nâlekame, tâpsomataur

Mâne pilorigutigivâ,

Inôlune tâpsomane;

Tussudlartuble Piulije

Erkitsomavlugo takpâne, 
Merngoêrvingmut pivoktauk:

Tagva akkisuktitaulârpoк Anerninga kiksarkattartok Tussujorlo saimanermik.

\section{Mel. 58.}

Wie wohl, o Jesu! wird uns. *1713.

1. Illa pilorigomârpogut

Jêsuse! igvit tækkuksaunak

Nagligidlartavut

Mâne tamainit,

Okerpervigijavullo ômamut,

Tautukuptigit!

2. Sakkerviksat nelliutikpat Illagêt illaliorungne, Kanortôk Jêsuse! Âkiksortigut,

Itterkovluta kuviasugvingnut

Najugarnut.

\section{Mel. 9.}

Wenn ein Herze merken kann. 1185.

1. Inûb missigiguniuk, Angergarviksane, Jêsus aivigilugo Tuksiarvigivâ.

2. Imâglo okautivâ: Merngortudlarama
Ailaungmiuk tarniga, Angergartidlunga.

3. TarningêrtoK timiga

Merngo ̂̂rsilerpor Ivjume, ômarviksak Nelliuxârtinnago.

4. Innigijomârivâ Ama tarningata Jêsuse tautuklugo Nungusuitomut.

\section{5๊1. Mel. 230.}

Wo seit so viel hundert Jahren. 1186.

Jârit hunderdit unnuktut Okpertut tamât pivinginut,

Angijullo mikkijullo

Ailerput, nâksaungitomik Inôniarlutik, Gûdemik

Nagliktut, nâletsiartullo, Tagva illûnatik Tækkolerpât tâmna, Naglingnermut Pângat pijor, Tokkolauktok, Pivluta illuvertaujor.

\section{Mel. 23.}

Du verlangest mich hinüber. 1188.

1. Jêsusiga kaikogamga, Tagva tamanêlerpunga! 
Nellopкotigal Гoartunga Takopsoagilaungminga.

2. Tamaita kattangutikka Illingnut perkogivakka, Kemmakapkit, tâpkonunga

Ullapкoteкartiminga.

\section{8อ๊อ. Mel. 168.}

Bei Dir, Jesu, will ich bleiben. 1193.

1. Jêsusiga najornerne

Itsainaromavunga,

Pairitsainalaungmingatôk

Pattangatsainarama;

Kanoênimnik tamainik

Okautênaromavagit,

Tuksiarvigivlutit:

Ârilaungminga aungnut.

2. Illingnullo okpernermut

Ungatsainalaulanga;

'Timigalo tarnigalo

Idluarsalaungmigik:

Angergarkolârumgalo

Nunamêtsungnaerko-

$$
\text { lunga, }
$$

Kugviorungnaipunga

Najornerne - ikuma.

\section{Mel. 58.}

Ich werd' mich über mein. 1194.

1. Tarnima piulijauninga Opigosumut erkarpara
TattamnartoKsôngmat

Piulijima

Tammarkattaralloartillunga

Nâpkigingmanga.

2. Tоккокârtinanga

Nâlegax Jêsus!

Mannigortigitsainarlagittôk

Tautuklutit,

3. Âniatillutit

Tokkolutillo

Aullo ajungitor kovigangne,

Piulivlunga

4. Kuviasulârpunga

Angidlartomik,

Killangme ikkingne merngoêruma

Sullinimnit!

5. Tussarneromârpok,

Takpânemiut,

Piulimajattit illûnatik

Tuksiarpatta:

6. „Jêsuse pivluta

"Tokкotaugavit,

"Ômamut nakoridlarpaptigit

"Sorairata."

7. Nâlegak illûnatik Mattomanêtut 
Kilangmêtullo nakorilitsuk,

Amen illa.

855. Mel. 164.

Nun Herr, der Du im Thränenthal. 799 .

1. Keanartovingme mâne Gûd illannâkarnernut Tækkonârnivit illume Nekkoksitimmattigut, KuvianadlartoK
Najugarnêtorle,

Pâne pigijomârtavut,

Mâne ikpigilerpavut.

2. Tamapta âKiksortigut,

Mâne inôtilluta

Nakorilutit ômamut, Paunga perkârtinnata Kênavit sânganut; Tarnivut timivut Ivsornaititautsainarlit Pâne illaukârtinnatạ.

\section{ANGERGARSIMAJUNIK ILLAGEKTUNIK, NAK- SAUNGITOMIGLO ÂNANAUNERMIK.}

85็6. Mel. 152.

Liebet Gott, o lieben Leut'. *1732.

1. Gûde erdligilugo

Nâlengniarsiuk,

Inuit Engelillo

Nâkoridlarsiuk :

Kennertane nuname

Paritsiarmagit,

Angergautijanelo

Kuviasuktipait.

2. Pakkoa angergartut

Pilorikput illa,

Kuvianartovingmut

Ittersimagamik.

Mâne nachogijauvut

Okpingitunulle,

Mânna Kuviasukput

IssoKangitomut.
3. Kiksartitaukattartut Angergarsimavut, Gûdib sânganut pivut Piulimajunut. Ômatitik ubvarpait Jêsusib aunganut, Kugvingit allarterpait Kôktut ijinginit.

4. Pakkoa attigivait Annorât kakkortat Immerungniangilat Kângniangilallo. Jêsub каiкopattigut Illagêngnerminut Nellipsarominarpok Nuna Kemmaklugo.

5. Amitor apкosineк Attorniarlavut, 
Kilangmut illingangmat

Illa inôtsemut.

Jêsusib inôsinga

Taimak malliklavut,

Igsarasuarlugo

Adsigêgomamut.

6. Kigligiutigilavut

TokKonît inungnut,

Tarningillo illingnut

Kajungersarlugit;

Jêsuse ânianît

Tættigigaptigo,

Tamapta kaititigut

Takpaunga illingnut.

\section{5\%. Mel. 97.}

Wie freut sich doch mein ganzer Sinn. 1197.

1. Illuêngardlunga tagva

Kuviasudlalerpunga,

Gûb kittornganginut mânna

Jêsusib illautimmanga!

Taimaimat ajortut illûnaita,

Ômamut tamât ipperarpakka,

*2. Taimaidlarmat, okpernikut

Sâlauvut suksaukôngitut; Utakkivakka ânanât Kilangme pânêtut nutât;
Saugârsûb aunganut tamakkoa

Illûnaita kingormgutivakka.

\section{5๊8. Mel. 205.}

Amen ja, Halleluja. 1199.

1. Kujanak, kuvianak!

Jêsuse nertorpaptigit,

Illingne, najugarne

InniksaKarkogaptigut;

Illûnatik okpertut

Kuviagidlarlitsuk

Takpâne sorairata

Nâlekamêlârapta.

2. Illagêt - pigâritse!

Opallungaitsainarluse, NâlegaK - Kannidlarmat;

Kattititaulervialo

Okpertorotiminut

Âkiksortaungmat mânna,

Nipliavigisiuk:

Kait Nâlegar Jêsuse!

\section{5ீ9. Mel. 119.}

Hätten wir nichts als. 1200.

1. InôseK - nuname

Pituariguptigo

Issumagitsænnagolo

Inôsek nâksaungitok,

Inôluta kissiane

Kristuse nerriuginago,

Suksauvâ? :,: 
2. Aukagle! - ovane Kugviorvekarpogut; Pâne kattersolârpavut Mâne pinniartavut; Inôjor nertornartomik Itterpok kuvianartomut, Kilangmut. :,:

3. Serrile :,:

Tarne piulijaujor

Taimak Jêsusib pivâtit

Mânna upkuêrtauvok

Kilak, Gûdib tikkipâtit

Time kemaklugo âpâtit

Ingminut. :,:

4. Kaumajok :,:

Mânna sakkijâlerpok

Hallêlujâleritsetôk!

Kaumajub kittorngangit

Engelit illagilugit

Jêsus nipliavigisiuk!

Kaumajok.:,:

\section{Mel. 208.}

Selige Gredanken. 1202.

1. Nekkoksinartojor

Nerriugijavut

Sokкosingitok!

Jêsub najugânut

Itteromârtogut

Tækkolerlugo,

PânetoK serrinartok;

Mânelo inôtilluta

Ungagênarlugo.
2. Pâne Jêsusiga

Kemmergolerlugo

Nagliktigêmut,

Ajornimnit mâne

Piulingmangale

Âniaminut,

Timemik ikkelingmik

Kunniklunga tækkonermut

Kuvianartotôvor.

\section{Mel. 9.}

O Jerusalem, du Stadt. 1208.

1. Illagêt takpânêtut!

Uiksan pivasse

Nerriugidlartavut

Mânna tækkovasse.

2. Kanor illingavise

Piulimajose?

Kugviorungnailuse,

Taututsainarmase.

3. Inôtsialauritse

Merngoerserluse, Tikkitsiarivlugit.

Okpertokattise.

4. Sulle nunamêpogut

Kiksarkattarvingme.

Kuviagijanganut

Âkiktaukovluta.

5. Tumise attorpavut

Sivorliojose, Mittautigijaunise

Tukkisigivavut. 
6. Issumakârtinnase

Salutilerpapse,

Kunniklugit ikkingit

Sorlo pigapsigik.

7. Takpâne attautsemik

Inniksakarpogut

Gûd soraijuitomik

Nakoridlarpavut.

\section{Mel. 15.}

Gehabt euch in der Heinat. 1209.

Kuviasugitse kilangme!

Nunamêtogut sulle,

Jêsub itterkojininga

Paunga uttakkivavut.

\section{6ீอ. Mel. 184 a.}

$O$ angenehme Augenblicke. 1211.

1. Kuvianamêk nelliutor!

Nerriungnermik sakkêјок,
Angergarkojaularaptatauk

Paunga piulimajunut

Hailig, Hailig, Hailigêrtunut!

Pilorênartuksaugapta,

Tâmnalo taututsainarlugo

Aungmut ullapkosertivut.

2. KuvianaK allianamêk!

Kaumanek ussornadlartok!

Kilait illûnatik sekriningat

Kaumaksaromârpattigut, Piguptigo imgerusertâk, Gûd tækkuksaulertillugo, Nertoruptigolo Saugârsuk

Tokкotaulauktoк pivluta!

\section{IMGERUTINIK SORAIRUTIKSANIK.}

\section{Mel. 164.}

Der Herr gesegne und behüt'. 878 .

Nâlekab sapputiluta

Pitsartutilitigut!

Nâlekab saimanerminut

Kaumaksarlitigullo!

Nâlekab Gûdipta,

Illagêktitane

Mânna tækkonâlauligit

Ullapirsautekartiligit.
865. Mel. 22 a.

Die Gnade des Herrn Jesu Christ. 185.

Kristuse nâpkigittigut! Atâta nagligitigut! Anernerlo ajunginek Illagitsainarmitigut. 
865 ๑. Mel. $167 \mathrm{~g}$.

May the grace of Christ our Saviour. 208.

Jêsusipta saimaninga, Atâtab naglingninga, Anernerub illagêngninga Missigitsainarlavut!

Tagva inôniarpogut Nagliktigêdlarluta, Piksaxarluta takpângat Kuvianadlartunik.

\section{Mel. 8.}

Ach bleib mit Deiner Gnade. 695 .

1. Nâpkiningnernut Jêsus

Najutsainartigut

Arкonartauronnata

Ômigijiptingnut.
2. Orautsitit Piulijik Uvaptingnênarlit! Piloridlarkovluta Ovane pânelo.

3. Kamanernut каumajoк Illagênartigut! Tammartailitilitigut MikseKârnivîttôk!

4. Saimanernut Nâlegak Pitsartutitigut! Ajungitut tamaita. Agliserkovlugit.

5. Nertornarnit Gûdivut Sakкijâtsainarle! Ajortunit tamainit Piulilauktigut.

\section{IMGERÛTINIK SAIMARTITSINERMIK.}

\section{(a) Attautsemut ILIINGAJUNiK.}

86\%. Mel. 79.

1. Aulik Piulijivut Saimartidlalaungmiuk Una illavuttôk, Ômatinga kibliguk Timingnut ikkilingmut. Nâlegar tussartiguttôx!

2. Ômat nertornadlartor Pivluta ikkilertar
Pitsartutilauruk,

Ikkivit iptingænnut

Pitsartuningænnullo, Tarninga pijariakarmat.

868. Mel. 10.

1. Aungnut ubvarsimajor Sokrosijuititsuk 


\section{(a) Attautsemut illingajunik.}

Unganerme illingnut,

Perкojarniк nâlektitsuk!

2. Tarninga inûliuk Umatâ kibliklugo, Issumangit illingnut Torârtitsainalaukit.

3. Inôtillugo mâne.

Nangianartovingme Serngnigitsainalauruk Ajortunit tamainit.

\section{Mel. 22.}

1. Aungnut pissiarigangne Ubvalauruk ômatinga, Ârilaungmiuk tokrungnut,

Akkiksaunovlugo aungnut.

2. Nutâmik attitilauruk Gûb idluarijanganik Piniutigilauktarnik Tokrungnut âniarnullo.
3. Illagêktut akkorngænne KivgartorKovlugo sângne, Aiparitsainalauruktôk Tamaine ikkajorlugo.

870. Mel. 11 a.

1. Jêsus piuligangne, Illavut una, sângne Inôle, âkilauruk

Tamât nertornautingnut.

2. Nunamêninganelo Pairitsainalaungmiuk, Tessioruk aggangnut, Pêrkonnago illingnit.

3. Kivgartorkolerangne Pijariakartanganik Tunnitsivigilauruk Ikkajorlugo tamât.

4. Pitsartunernut Jêsus! Ajugautilauruktôk Nukkeкanginingane, Tættituaringmattit.

(b) MAgGUNGNUT.

8\%1. Mel. 79.

Aungnut erdlingnartomut IdluarsalaukiktôK

Perkutigigangne, Kajungertitsainakik Illingnut sorairatik Inônasuarkovlugik.
872. Mel. 23.

1. Naglingnernut ômatigik Ônarsitikik nutâmik, Tessiulaukik aggangnut Piuligangne torkungnut.

2. Kaut tamât serngnigilaukik 
Támmarkonnagik illingnit,

Аркоmele amitome

Arvertarkovlugik sângne.

3. Tarnigik ivsornaitikik

Ungatilugik illingnut.

Angernitik malliklugik

Sokkosijuerkovlugik.

\section{Mel. 10.}

1. Jêsusiga! illingnut

Mânna tuksiupavut Saimarlugik pairikik Mattomanêtillugik.

2. Ômamiglo nutâmik Tunnitsivigilaukik,
Asserorsimanigik

Tokkungnut tokkotikik.

3. Sujungaitilaungmigik Idluaringitarnit, Pigannertilugiglo Unganerme illingnut.

\section{Mel. 164.}

Kattangutîkput ukkoa. Aungnut serparsalaukik! Saimarsainernut kaut tamât

Kolangiarvigikik; Kiksaleraikpannik Mannigorsẩlaukik,

Najornivit nekkoksinartub Illagitsainalauligik.

\section{(c) UNUKTUNUT.}

875. Mel. 79.

1. Piulijivut aulik Saimartidlalaungmigit Ukkoa illavut, Ômatingit kiblikit, Timingnut ikkilingmut, Nâlegar tuasalauktigut!

2. Kattangutit ukkoa Pivlugit kennugapta, Jêsus! nâpkigikit, Aumatikit tokkungnut, Inûlilugit aungnut, Ungatikillo illingnut.
3. Aggangnut puttolingnut. Tessiutsainakittôk. Saimasârlugillo, Innigikit Jêsuse! Ivsornaitikit sângne, Naglingnernut kiblilaukit。

4. Idluarsitilaukit

Âniarnut aungnullo, Inûlilugillo

Ikkingnut, Anernernut Tessiokit, naglingnernut. Ônarsitsainarkovlugit. 
(c) Unuktunut.

876. Mel. 10.

1. Tungatikit tokkungnut Âniarnullo aungnut, Innigitsainarlugit Pêrkonnagit illingnit.

2. Nangminer kennerangne, Tagva illagêktune Pivalliakovlugit Aungnut serparsalaukit;

3. Aglisidlartilugit Aungnik iptekartikit Tessiokit tamainut Idluarijarnuttôk!

87\%. Mel. 146.

1. Gûde tessiokit, Inôtseme nuname, Kemangniarnagit, Sapputitsainakille, Pairikillo, aungnut Pissiarigangne, Inôlitt tokKungnut AngergarKâratik.

2. Kaumartitsinernut Illitarksitilaukit Pattanganermingnik Piuliklernerniglo ; Tungatilaungmigit Tokrungnut aungnullo, Sokkoserkonnagit Inukotingnele.
878. Mel. 167.

Kivgaujungnaitilaungmigit"

Idluitunut aungnut,

Pinasuartilugillo

Ivsornailinermingnik;

Illingnut inôKovlugit

Idluarijarnullo,

NoKkalerangatta, tagva

Tuppaktitsainalaukit.

\section{Mel. 159.}

1. Ômatingit rellaksukit

Ômatingnut Jêsus!

Nâletsiartilugillo

Ajorertûsernik.

Nangminer tessiulaukit Aprotingne manniktome, Ajokertulaungmigillo Idluarijarnik.

2. Jêsusivut angerningit Tussajarêrangne Sokкosijuitilaukit Pitsartudlarnernut. Illingnit sapkutailikit, Pairikit nertornartomik, Issumatit tâpkonunga Nâmaksekârnagit.

3. Ovunga aiklaurangne, KivgartorKovlugit IllagêktoKotingnele, Ikkajutsainakit; Pijangit aulatilaukit, 
Pairiuk arvertarningat Igsagaksangortidlugit IllagêKotingnut.

880. Mel. 106.

Atterne Gud ingergarmalle (mannik, matta)
Illagitsainalaungmiuk

$$
\text { (gik, git) }
$$

Engelikut sapputilauruk (kik, kit)

Nangianartokalerpat,

Kanoênane (tik) perkovlugo (gik, git)

Tillijauvigijaminut (mingnut).

\section{(d) SORRUTSEMUT ATTAUTSEMUT.}

881. Mel. 58.

Sorrutsinik naglingniktotit

Jêsuse pairrsijôtôgavit,
Nâpkigitsainaruk

Nutarak una,

Perortilugo illingnut illa Kittorngaule.

(e) SORRUTSINUT ATTAUTSEUNGITUNUT.

\section{Mel. 164.}

1. Nagliktor Jêsuse! igvit Sorrutsit naglikangne. Illingnut perortilaukit Kuviagijarnuttôk;

Pairitsainalaukit, Agliserkovlugit, Illingnut inôkovlugit,
Kilangmut каікокârnagit.

2. Jêsusib Piulijipse Erkilise nutâmik, Aungminullo ubvarlise Inôsertârtilise, Kuriagijanganut Illingakovluse;

Nellipsarsiuk uvlome, Nâpkigijomadlarmase. 


\section{NIP PISI0R UTIT}

VERSIT ILLÛNAITA, AGLANGNE TAMAKKONANÊTUT PIVLUGTT.

\section{A.}

Abba Atâtamnik! mânna 239 Ablatsângortitaujomavl. 492 Abrahamut angilauкрок 23 Adamekut assiojilauktam 43 tammarapta 23 Adsigilerluta aglât. . 57 Adsigijomagaptigit . . 679 Aggakpîllo saimarpânga 375 Aggaktit puttomajut . 594 puttortaumajut 755 Aggangillo ittiggangillo 130 Aggangne Gûd innilanga 306 Aggangnut pitsartujunut 721

” puttolingnut 875
nut . . . . 758 Aggangnut puttosimajunut 406 Aggangnut tessiortigut 194 tigulaunga 475 tigumiænga 454 Aggü Jêsuse Gûdiga . 21 Jêsuse pingipat 385 kovilaurangne ôm. 670 kovilaurangne suj. 481 Aglait hailigit illa . . 6 Aglaksimavor illingnik 664
Aglisidlartilugit aung. 876 Aglisitsainarluta inô. $\quad 513$ Agganne puttoggit Jêsub 732 Ahâgle Nâlegar Jêsuse! 289 Ahâk Jêsuse! kittorngat. 239 " Jêsuse sângnut 220 " Pairijiga saugârs. 432 " tamânêpunga . 88 , tarniga kêdlartok 505 Ahamarik ajortokullôvung. . . . . 353 Ahamarik! Gûd окагрок aj. . . . . 207 Ahamarik! Gûd oKarp. pij. . . . . 209 Aileruma perorsêvingmut . . . 130 Aipânik inuartomut . 116 Aiparêk sivorlerpâk . 696 Aiparilaulagit Gûdiga! 95 Aiparilaulagittôk! Get. 132 Aiparijomavagit Golgatam. . . . 387 AiparitsainaromadlarAiparut tækkuksaulugo . . . 386 jungnai. . . . 707 Aititse nellojut nunang. 806 Ajokertolaunga Kuvia. 219 Ajoksarnartovingmênap-

ta. . . . 199 
Nippisiorutit.

Ajorsatsainarluta pin. 212 Ajoksatsainarnivulle . 706 Ajoktarnik nellungilar 173 Ajorama anernernut . 495 Ajorneк illume . . . 211 Ajornernik ikpigijor . 45 Ajornekangitotit Jêsus 696 Ajornerit allarlavut . 93 Ajornikka illa unnuk. 224 pivlugit kapp. 114 pivlugit sior. 137 tamaita. . . .262 Ajornikkalo tamaita $\quad .109$ Ajornima miklitipânga 463 Ajornimnit ubvarmanga $2 \%$ Ajornimnut tokKung . 246 Ajornipta nênermatsuk 93 Ajorniptingnut tokкung. 24 Ajornise pivlugit sannik 30 Ajornivullo tamaita . 279 Ajornivut pêjalaukittôk 562 Ajornivut pêjarmagit . 39 Ajorsatsainarpungale . 451 Ajortoliksôjunille ern. . 217 Ajortorsôjogut illa . . 464 Ajortorsôvluta . . . 16 Ajortorsôvogut omi. . 215 Ajortorsôvunga nellaj. 462 illa . 239

AjortoKullôjosetôk . . 208 Ajortomik akkuneкa . 257 Ajortorlo illumnêtor . 488 Ajortoselo illipse . $42 \mathrm{c}$. Ajortose tækkosiuktôk 212 Ajortuggit naglingmagit 559 Ajortunga pigivarma . 508 Ajortunit ajortûnersau. 211
Ajortunut unganermit 671 Ajortut nênidlalerp-

Ajortut ômatinginut $\cdot 13$ pitlartaujutitit 98 Ajoruma kannimamut . 123 Ajugaujor kujagijartor 151 Ajudlarpunga nel. . . 278 Ajulênadlartunga . . . 35̃4 Ajulernera tagva . . 245 Ajulernivut angijok $\because 352$ Ajulertunik tamainik . 676 Ajungitorsoak Jêsub. . 72 Ajungitamnut tamainut 322 Ajungitullinikkalo ass. 17 Ajungitullinît pil. . . 339 Âkigomavlutale . . . 539 Akkerartortulle . . . 92 Akkerartortut illingnik 4 Akkerkotut illa . . 628 Akkillêvigilutit, Jêsusiga 134

Akkinernilk ajorpunga 1107 Akkisartuivlune . . . 27 Akkonaptingnêkuvit . 81 AkkonaptingnelerittôK . 582 Akkonaptingne unêt . 608 Aklugalloartub . . $47 \mathrm{a}$. Aklujokullôdlarama . 496 Aklulerlune tikkipor . 34 Aklunima Jêsusiga . . 505 Aklunima angijorsôning 509 Aktoleruma timimnik . 71 Allagaigilaukpakka . 137 AllarniarnangatôK . 831 Allarlavut . . . 537 a. Allaromangilara . . . 420 
Allaromangimanga . . 838 Allaromangivâse . . . 31 Alliagidlarpara . . . 421 Ama tækkolârpaptigit . 167 " tækkonâlaunga . 134 " tækkonâraminga . 257 Amen Abba Jehôva . 190 " illa Hallêluja . 709 " illa nertudlar . 365 " kiblingnartomik 555 Amerkotuu-ipallônêt . 211 Amitôk apкosineк . . 856 Anânatut najuênanga . 435 Ânanaujor Nâlegar . 736 Ânanaunermut Nalegax 346 Ânanaunerpângojotit . 499 ÂnanaunersoaK . . . 20 Anernek Gûde . . . 465 Gudiojotit . 179 " Jêsub . . . 735

" naglingniktotit $472 \mathrm{a}$.

" ômartitsijok 200 " pângat . . . 445 $"$ piniarnitit . 200 " tokкungajunik 630 Anernængerkârtinnanga 257 Anernængertok Nâlegar 145 Anernerlo ajunginek 178 Anernermut erniango 441 Anernernut najunga . 768 Anernerpîllo timiga . 326 Anernerpît innerteri. . 470

, tuksiartitima

" tuksiulinga
Anerniojut

Anernîk Jêsub

797

Anernipkut Jêsub . . 144

Anerniptigut aggaktit . 582 Anernît ajunginer . . 9 Anernivut kiblikit . . 549 Ânertaulauktotit . . 698 Angergarкojaukârnanga 606 Angergartittigut . . . 827 Angidlarmat oKautau 629 Angijor Jêsub attinga 545 Angijomik kangusukpogut . . . 187 kuviasukp. $\quad 826$ Angijunut mikkijunut 553 Angmarkojominadlarрок. . . . 427 Angnerpâk mittautaung. 137 AngnerpângojoK . . . 154 Angut ânialik Kem. . 298 " ânialik mâne . 295 " ikkilik . . . 399 Angutîgle magguk . . 160 Âniadlarkârvingnit . . 132 Âniagavit kiæguk . . 733 \# Jêsuse . . 226 Âniajotit tessiulaukt. $\quad 669$ Ânianadlartomut . . 30 Ânianartomik. . . . 432 Ânianga ômanartomik 130 A . pivluta . . . 79 Âniangit ikpigi. , . 399 Âniangita kissimik . . 412 Ânialik nagliktor . . 145 Ânianitit kikiaktor. . 102 nênikit . . 649 744 a. Âniarnut tamainut . . . 428 
Âniarnut tokkungnullo 376 Âniatigingmanga .. . . 259 Âniatillutit . . . . 854 Aniatitaugaville . . . 138 Âniavigijarnut . . . 572 Aniavîllo akkiksang. . 103 Âniavinginut tamainut 99 Âniat Jêsusiga . . . 110 Annerijaumanivut . . 315 Annerijipta Jêsub . . 314 Annerimanivut . . 312 Anneringmattigut . . 359 Annersâtainarniptingnit 21 Annerterigârnerpît . . 834 Annikitotuinarmigle . 159 Annoraijarlugolo . . 92 Аркоme amitome - 537 a. Apkutauvungalo . . . 165 Apputitut niglingnarp . 211 ArlingnartoKsoak 72 Ârlorpogut takpaunga - 297 Arnak кeadlartor . . 298 Arngnaungub piarkane 795 Avertartilaungmittig . 679 Arvertatsainaromagama 681 Assezutsijut issumait . 707 Asserorniptingnit piul . 16 Asserorsimagapta ill . 670 Asserorsimanek sakrijât . . . . 686 Asserorsimanerminik . 261 Asserorsimanersoara . 511 Assianigle tætteкаrtoк 270 Assiminik tessiortuksar 723 Assingnut ômatiga . . 428 Assiojuksaukôrtorle . . 261 Assiojuksaunerminik . 211
Assiokonginamittigut . 244 Assiomalaukpunga . . 262 Atâta! tilliklirlauktotille 179 Atâtab Erninga Gûde. 34 naglingninga 184 issokangitub . 34 nangminek illa 165 sângane . . $42 \mathrm{~d}$. sânganit pivok 37 tallerpiane . . 737 tallerpianêpoк . 365 Atâtaga kittorngarnik . 173 Atâtagigapkit nutar . 177 Atâtagigaptigit Gûd . 189 Atâtak amuanga .. 744 a. , kilangmêtotit . 186 , tâmnale piuk . 276 Atâtaminut pitipait . 214 Atâtamnut paunga . . 165 paungarpunga 17 Atâtamut okaravit . . 239 Atâta! nertorpapti it . 179 " pairikit . . 654 Atâtangnut ailaukpotit 419 Atâtanga Ernerub . . 693 Atâtapta issumangit . 11 Atâtatun-ipotit . . . 25 Atâtaujub sorlo . . . 16 Atâtaujunit tussaum . 542 Atâtavit agganginut . 145 \#. sângane . . 745 Atâtavut kotsingnerme 28 serngnigênart 587 Attẩner nertornartotit 485a. Attannîk! kina illiktun 343 pijomajarnik 343 Attannit unnudlartut . 27 
Attausênak tagva . . 538 Attauser pijarialik illit 329 " Krist.

283

Attauserlônêt assiokon. 211 Attausingortittigut . . 438 AttausingortittiguttôK . 440 Attausiotênartigut . . 546 Attautsemik tussuluar-

punga . . 283a. Attê, annernîk, tarnîklo 810 " Jêsusiga tussakit 684 " nellojut, tuppa . 733 " tupaleritse - 42 a. Attêle nertorsiuk . . 151 Atterne Gûd ingergarmalle $\cdot \cdot \cdot \cdot 880$
ingergarapta .783
sullijæksakka. Attêtôr Gûde tamapta 64 64
27 " makkilerta. $\quad 27$ Attit nakorijaule . . 190 Attoruma nelliænnik . 527 Aukagle, mâne kugvior 859 Aukpît, aukpît kovim. . 376 " Gûb saugârsung 425 " kovijub timing 409 ", saimanartub .628 Auktojor Jêsusiga! . 436 Piulijiga . .140 Aulajeкattautigilakka . 129 Aulatsiningata . . 814b. Aulik Gub saugârsunga 750 "Jêsusiga _. . 361 " Jêsuse! pigitigulle 792
Aulik Nâlegak, pivlunga 278 nâpkigijiga • . 354 Piulijivut illitari 731 Piulijivut saimar. 867 Piulijivut tamat. 740 sennerkab ikkinga . . . . 153 Aullo idluartoksoak . 427 Aumavunga tækkogapko. . . . 100 Aunâdlartunut ikkingnut 376 Aunârnernut ômativut 697 Aungêrotileruma. . . 124 Aungêrotor time, kanoK 142 " time, piksa. 127 Aungmiglo kidsijârner. 487 Aungmik immermiglo 130 " Kugvioravit 110 Aungmut pissiarilaung. 808 Aungnut erdlingnart. 871 " Jêsusiga illi. 404 " Jêsusiga najor. 790 " ningautsau. . 220 " ômativut iv. . 493 " pissiarigamg. 413 " pissiarigangne 869 " piulijattit . . 635 " piuligaptigut 299

" serparsarlaukti . . . .658 ubvarsima .868 Aut" kovijok pivlugo .520 " immerpavut . . 639

" illûnât ivlerinago 120 " pivlugo kilak . . 235 A"vatikka Kuviasugitse 62 
Avatingita puttortau-

ning. . • . 760

Avê Kattaktitotille • . 126

\section{IB.}

Baptijutikut Gûmut . 843 Betaniamiut anernipkut 87 Betania ullapirsautelik 87 Betlehem Golgatalo . 368 Betlehemele Dâvidib 31 Betlehem ivlernartotit 51

\section{且.}

Dâvidib erniarsunga 54 a.

\section{W.}

Êjomadlartiptingniglo $\quad 529$ Engelib tautua . . 56 tagva tikkipa 93 Engelit illagivlugit Piul. 653 illagivlugit . . 842 " $\quad$ kilangmit pijut 31 " pânêtut . . 47 a. " pitsartujose $42 \mathrm{c}$. " tillidlarlugit . 787 Engelivaksuit nertordlert. . . . 800 Eprêjarvigilaunga piung. . . . 467 Erdligidlartara nelleкang. . . . . 284
Erdlingnadlartorlo . . 72 Erdlingnarnerpângojor 558 Erdlingnartor attannivut . . . . 755 Erkailertuksauvungale 47 Erkarapko tamanna . 745 Erkarsiuk piluarlugo 71 Erkaumanadlarpok illa 155 Erkaumanartotuamik 608 Erkeasujuititigut, kajun. 718 Erkitsomadlarpagit . 393 Erssinartor imâgle . 829 Ermgusiarsuk Wainelik 609 Ernek, Gûd issokangitok . . 159

Ernerub Atâta nâlekpâ ' 17 Erniangoкârtinnanga . 47 Erksuklugo seningajo-

lik . . . 130

Erniangokârtox nuname 24 Ernine ivlerinago . . 185 Ernît tunigangne pivluta 187

\section{Gr.}

Glôria Hallêluja! · . 373 Gûb Anerninga kennu. 199 Anerninga! nagl. 203 Anerninga nert. 202 Anerningat kiss. 256 aggangit. . . 519 a. apkutâ attut . . 174 Atâtab Anernerublo . . . . 181 Erninga nagliktok 615 Erninga! tamainik 193 
Gûb Ernine naglingnermut illagêktoKotine 39 illingatitsiarpait 519 a. kittorngaringmanga . . . 647 Kuviagingitangit . 621 nâpkigijomatsangilait 270 naglingninga . 8 nagligosuktotôjub 812 nelliuninga neli . 640 orausinga imaip . 8 okausingine tamai-

ne orausingit nâlekit pissiarivânga . 370 pitsiarninga - $172 \mathrm{e}$ sângane inuk . . 312 sauganga illa. . 287 sauganga nâleklugo saugangata piulik saugârsunga anni saugârsunga piuli 195 sillaksoarmiut. . 59 sullijangit - issuma . . . . 523 sullijangit - piuli 523 tækkonârpait . . 23 Gû̉d allaromangilara . 531 Anernek ômamut $20 t$ Anernek piginga 486 Anernek, uvagut 276 Atâtak! pijatit . 75 attertut . . . 2 inuit kinauningit $172 \mathrm{~b}$.
Gûd, Gûdivut kattang. 55 Gûdivut nellek . 54 Gûdivut, saimart 607 ikkilersimajor . . 839 Kennuvigivara . . 520 kivgavit pijangit . 724 kujagivaptigit . . 204 nagligigangne in. 187 naglingnivit tor. . 94 nakoridlartaule . 29 nakorilavut ôm. . 805 nâlegaviksôngmat 75 nertorlavut! . . 542 nertortaule . . 148 pairikit tamât. . 589 pingasôlijotit igv. 180 pingortitiga . . 233 piviksakarkojikpat

519 a. saimarsaijotôgavit 217 sângne sakkersi . 18 sennalaurit illumne 217 tikkipaptigut tagva 252 tokkungmat piv. . 688 uvangale kittorng. 46 uvaptingnêpor кen. . . . 809 uvaptingnêpok, tâm. . . . 809 uvaptingnêpor, ul. 580 Gû̉de ajugakangitok .28 Anernernut tessior . . . . 224 Atâta Ernerlo . 771 erdligilugo . . 856 kemangilara . . 772 kujagilavut opigi 782 
Gûde kujagilavut inung. $158 \mathrm{~b}$.

" malungnartomik 171

" nakoridlarlavut Átâta . . . 803

"nakoridlarlavut Kuj. 178

" nakoridlarlavut mânna

" nakoridlarlavut ti. 642

„ nâletsiartomik . 756

" nâpkigosuktor . 184

" nertutsainarlavut 602

" nutaraujotit . . 42

" ômatiga . . . 276

" opigidlarlavut 149 a.

" perorsêvingme . 82

" sângnut pâmakp. 21

" saimartilaukit . 777

" sapputsauviksauvoK . . . 529 sittijoksônera .276 taimaitsainarlanga . . . 125 tessiukit . . 877 tussartigut . . 805 Gû̉demik tætteкartor . 537 tættetuakartok 174 Gûdemut kittorngaujose 166 Gûdeviksôjotit . . . 809 Gûdib ajokertûsingit 11 " l ajortônerpât $\quad \begin{array}{rr}184 \\ \text { " kattaksimanera }\end{array}$ anera 17 " kittorngangit . 233 " kittorngangita 205 $"$ nachogigunigit . 45 " naglingninga . 20
Gûdib ningarungnaer-

ninga . . 54 a. ningausinga illa 737 ningausinga mân-

na . . . . 252

okausingit illa . 5

piniarninga tar. 176

pitsartuninganut 154 saimaninganut 537 a. sânganut piguma 267 saugârsunga tun. 152 saugârsunga piks. 404 tunnergutingit .781 ullapirsautinga . 305 Gûdible Anerninga . . 205 \# saugârsungat . 205 Gûdiga Golgatame . 143 , inôtillunga . . 803 tuksiarpagit . 21 Gûdìk rellarudjâlaunga 45 כ nâpkigosungnernik . 209 Gûdipta oxausingit . 6 " saugârsunga . 577 Gûdiptalo Anerningat 783 Gûdivullo okarajarkâ . 534 Gûdivut inûlernît . 69 Jehôva . . 806 " $\quad$ kujagitsainar. 1 " tamainik ping. 657 , tættigiluaruk 68 Gûmik ijersimajomik $172 \mathrm{~g}$. nellâgut . . . 543 Gûmut illagêngojose · 630 nellopkotijunga 329 pititaumajut

" sennaviogupta $172 \mathrm{e}$ 


\section{㽬。}

Hailig Gûd Gûd . . . 1

Hallêluja Amen illa • 7 Gûd Anernek 182 Kristuse ômarm. 147 nakudlartub .182 nâleklugo G'ûd 182 nâleklugo nert.

182 nertordlerneк 154 nertortaule 706 Piulije . . 154 sorsungnivut $54 \mathrm{a}$. Hallêlujamik tuks. . . 509 Hallêlujârvigidlarlagit . 320

\section{面。}

Idluaringitatit

279

Idluarkutiksainarnik 519a. Idluarniptingnuungitoк 36 Idluarsaivotit . . $172 \mathrm{a}$. Idluarsilaungminga $\bullet 457$ Idluarsitilaukit . . . 875 Idluarsitilaungminga . 276 Idluarsivok ômat . . 482 Idluartub Gûb aungat. 374 Idluinima nênerlutit . 98 Idluinerpângomangâpse 211 Idluitullijotut annau . 102 Igville tamainit . $305 \mathrm{a}$. Igvit Jêsus! pituari . 274 " kênak annerimajotit. . . . . 131
Igvit Kejungmêlutit . . 387 " salutitau . . . 32 , tamapta kittorng. 78 Ijik kanningalo . . 822 Ijîkka kemmergoleritikko . . . . 131 sikkungipannik .788 Ijîmnut ukkungnunga . 838 Ijîngma . . . . 283 a. Ijîngminut sangêtungnut . . . . 257 Ijîngnillo kugvitit . . 391 Ikkajortaujomavluta .698 Ikkinginêleritse . . . 593 Ikkinginit kôktomulle . 632 Ikkingit pivlugit. . . 122 tautukaptigit . 398 Ikkinginulle perko. .579 Ikkingne Gûd, innilang. 747 Ikkingnêniarpunga . . 132 Ikkingnut aupallukt . 842 Ikkitillo sorainanga. . 390 Ikkitit aitangajut . . 158 Jêsusiga . . . . 769 Kaitsingmatta . 222 kemmergogupkit . . . . 390 kunnidlarlugit . 392 pivlugit ajaktorn. tækkogupkit Kug. 158 tækkogupkit kuv.158 Ikkivit aunârningit . . . 554 Ikkomamit Sâtanemit . 22 IkpeksaK uvlomelo . . 561 Ikpeksarlo uvlomelo . 173 Ikpigijakka tamaita . 238 
Ikpigijaptingnik mânna 6503 Ikpigingikuma asser . 479 Iksivagalloartotit mânna 346 Iksivautamut aititse . 808 Iksivautaksoarminit. $42 \mathrm{e}$. Illa aglât ôminar. • • 96 akkitudlartomut . 340 idluinikka . . . 218 ikkitit pivlugit. . 810 illanâkardlune . . 214 ivsornartoksôjok . 276 Jêsus Gûb erninga 64 Jêsuse tokkungnut 621 Jêsusiga nagl. . 42 a. Jêsus taimaile . . 717 kattakattarapta . 355 Kejungmut . . . 399 kiblingnartoKsôvok 672 kittorngatit. . $814 \mathrm{a}$. Kugviudlarnermut 376 kuviasudlalerpunga 106 Kuviasukpunga • 45 nagligivarma . 132 naglikpara . . 434 Nâlegavut kikkiak 431 nellonadlalauktut $172 \mathrm{~g}$. ômamine . . . . 385 оріnarpok . . . 351 piloridlarpunga igv. 309 piloridlarpunga

Kêm.

309 pilorigomârpogut . 849 sorruseK igvit . . 843 taimaitsainarpok . 410 tammarkattarama 478 tâmna pilorikpor . 307 tarne serridlarpox 307
Illa tættetuakartok . . 502

" tessiorluarpânga . 320

" timiga tagvane . 126

" uvanga tagva ... 110 uvlâmit unnungmut 93 Iilâle ajortavut . . . 184 " angidlartomik . 341 " angijomik . . . .55t " tagga illûnara . 280 IllanâK amuartigut . . 324 " ajortolingnik . 214 " kenuniptingnut 194 " ômatiga . . . 486 " pitsiadlartoк . 440 " tarninik . . . 351 Illanâkarnît illa . . . 670 IllanaKartok illa . . 5̌5̃5 Illanâra aipatuarigupko 325 Illanârijama uvlunga . 161 " tarninga . 130 Illanâritsainarminga . 290 Illanârnerpângôvotit . 499 Illanâtuarijama Gûdib . 135 Illanâtuarivagit . . 196 Illagêkluta mânna Gûd 37 Illagêkotitit aungnut . 623 Illagêktitatit aglisitikit 747 Illagêktitavit Kennuvâtit 578 IllagêktoKotitit Kom . 608 IllagêktoKotivit niak : 747 Illagêktose! Jêsub sai 579 okokse! . 414 Illagêktunut niakotô : 78 Illagêktut akkerngænne kiv. . . . . 869 Illagêktut akkorng. mânna . . . 356 
Illagêktut attê! nâgl. 446 inôtsiarput . 728 Kuviasugitse 598 okokse! . 110 tamaita nagl. 438 Illagêlerapta piniart. . 575 Illagêngnivut aul. . . 636 " saim. . . 743 Illagêngortitaujose . . 585 Illagêt akkunapsingne . 582 Gûb taimak. . 1 illa kuviasuk . 760 illagisiuk . . 83 illûnânemiut $\quad .728$ kattersorangne . 581 kemangnagit . 569 kugvise . . . 565 KuviasugitsePiu. 625 Kuviasugitse! tim. 625 mallitsainarsigikt. . . . . 556 serngnigiluakit. 544 serridlaritse . .820 serrititse . . 560 tækkonâkit . . 749 takpânêtut . . 861

Illagitigut Kristuse . $\quad 783$ Illagivlugit Piulijivut . 264 Illakkale okokse. . 795 Illavut tammarkonnagit 443 Illerasukonnatalo sângne

697

Illinganek ânanauvok . 510 Illingnêtsainaromavunga 511 Illingnêtsomavunga . . 53 Illingnêtsainartikit . . 675 Illingne pitakadlarpok. 499 Illingnik Gûde tamapta 491 " inuit ômisud. 211 " kivgartorko . 291 " ungadlarnera. 463 Illingnullo okpernermut 853 Illingnut adsiotinianga 408 " inôjomavunga 830 " okperpunga . 146 " Piulijiga!. . 617 " tarnivut perk. 5.94 Illinniartingit tækkonâr. 162 Illiornek tamanna . . 686 Illitarigupta nangmi. . 233 Illitarimarikluta Saug. . 659 Illisimajotôjotit sil. . . 216 " tama. 194 Illuêngardluta Gûmut . 564 Illuêngardlunga tagva . 857 Illumne anernera . . 218 " Kaumaksarlanga . . . 11 Illumne nâmagingi . $\quad .280$ " passijauvlunga 257 " sôg keadlarkik? 606 . sorlo tussalerp. 265 Illûnamnik pitipagit .11 
Illûnamnik tunnivunga . 721 Illûnâne Gûdiga . . . 176 Illûnapkut ajorpunga . 396 Illûnaptingnik tunnijom 669 Illûneralo inôseralo . . 673 Illûnarmale uvlom. . . 278 Illûnarsoatik inuit . . 260 Illûnasele illagêt! . 359 Illûnata opigukta . . 39 Illûnatik ajulertut akluj. 212 " âniatit . . . 374 $" \quad$ kiksartut . . 410 " okautsivut. . 653 Illungertornermulle . . 772 Illungertudlartok toк. . 130 Illupkut keavakpunga . 605 Illuptigut keagupta . 807 Illuptingnele ajortut . 698 Illuptingnêtut. . . 163 Illuserilaukțanginut. $172 \mathrm{~b}$. Illuvermullo illivât . . 737 Imâglo oxautivâ . . . 850 tuksiarmita . . 784 Imẩk oKautsaukovattit. 211 Imgerput Hôsianamik . 672 Immanuêliga! nægliguk 468 sapputiuk 821 Immanuêle Gûdivut 46 Immarbiuk kiglinganik 46 Immer tâmna tækkovâ 600 Immeruktut immikangne 419 Immerungmik nipleravit 842 Ingergarma Jêsusimnik 269 Innangalerangama . . 793 Innangalermiguma . . 407 Innigijomâriva . . . 850 Inniminit annijoк . . 25
Inôgutetôgavit am. . 29 Inôgutigijavut nelliu. . 563 Inôgutiksarsijiga . . . 113 Inôgutivut igvit . . . 78 Inôjut akkorngænne . 218 Inôkojomagumga sulle 830 Inôluarvekaromajub . 321 Inônata nangminiptingnik. 345

Inônermine nuname Jârille . . . . 36

Inônermine nuname kap. 93 Inôniarasuarit Jêsub .338 Inôniarkovlugik id. . . 693 Inôniarnernigle . . 176 Inôniarviksavullo . . 705 Inôser ikkinginne . . 313 „ illa nellagut . 266 " nuname pi. . . 859 ", pilloringnadlarp 498 " pigigaptigo . . 155 Inôsera illunaralo . . 96 " tamât Jêsuse . 333 Inôsiptingne nuname . 334 Inôsiptingnetôk illag. . 466 Inôsimne tamât . . 246 Inôsivut ijersimavor 531 a. Inôsungnimnit aglât . .524 Inôtigidlaraptigit . . 367 Inôtigigapkit illa . 196 Inôtillunga Gûdiga. . 94 Inôtillugo mâne . . . 868 Inôtsialauritse merng. 861 Inôtsib issua . . . . 432 Inôtsivut âkiksolauruk 489 Inôviksatuak Jêsub . 847 Inôvogulle perkojau. . 713 
Inôvunga torkosuert. . 319 Inûb ajorninele . . . 759 " missigiguniuk .850 " ôma niakoa . . 101 Inugle suksauvâ? . . 28 Inuille Gûdivut . . . 407 Inuit kattaksimajut . . 22 " nagliktut . . . 468 Inuk Gûdelo . . . . 195 " Jêsus kemergo: . 331 " piulijaunerminik . 154 " pivlugo Sabbate . 584 Inukangitomêkuma . . 496 Inukotitit illagêt . . 448 " Jêsuse . . 40 Inukullûb ômættâ $\cdot \begin{array}{r}\text { tamaita } \\ \text { ôm }\end{array}$ Inukutaujose Gûmut . 303 Inûlerkârtinnatale . . 808 Inûlernît Jêsuse, . . 53 Inûlijaunerma pijariak. 224 Inûlijiksamigle assianik 394 Inûliklerte tarninik . 194 Inûliktertub aunga . .637 Inumariolermat . . .663 Inungnik ajokertuijose. 2 " $\quad$ kaikojijotit . 46 " pairksigavit . 438 Inungnik sannituinarnik $17.2 \mathrm{~h}$.

Inungnut tamainut . . 648 Ipaksinatsangilarle Jêsub 682 Issokangitor Kaumajok 34 Issuma nautsertolauruk 491 Issumairotivungale sor-
Issumagijaujungnainermik 681 Issumagijungnaikit . $\quad 190$ Issumarkârtinase sul. $\quad 861$ Issumakka piniartakka 396 tuppaktikit 819 Issumaksarsiornanga $\quad .277$ Issumaksarsiortitigut . 681 Issumalinangitok Jêsub 244 Issumalingajoк inuk . 502 Issumalingasuêrnek Gûd 502 Jês. 502 Issumalingajuêrnermik 502 Issumalingasuêrnerle . 498 Issumamingnik inuit $172 \mathrm{~d}$ : Issumangnik âkilauruk 276 Issumatunata . . 809 Ittervigilauruk . . 305 a. Ittiggangnullo tagva . 669 Ittiggangnut mânna sêrk. . . . 667 Ittiggangnut sêrKort. . 246 Iviksukanêlertotit : . 32 Irsôgamale kangusuk. . 465 Ivsornaigannertikit . . 703 Ivsornainit idluarnillo 36 IvsornaitisimaneK nel. 494 Irsornaitub Gudipta . 364

\section{I.}

Jâreme mânna nung. . 78 Jâr nutaungitor kâng. “ 76 Jârit hunderdit unnuk. 851 Jehôva imgervigivagit 744 a. 629 
Jêsub ajokertûsingit 305 anganik ikpigi. 631 Anisningit kitkiak.

Aniangit opi. attingane . . $744 \mathrm{a}$ aungminut ineit 730 iklinginut . . 565 ilkingit angmar 633 ikkingit erzar. $\quad 762$ illagêrotingit . 621 illagêngita . . . 800 illuvermêlauxtub 154 illuvingane its. . 136 illuvingane sun. 139 inûlervia . . . 56 intuvia . . .42 d. ittigganginut pâm. 211 ittigganginut pA. 389 raumasingane . 699 kikkiaktortauvia 135 Kristusib attinga 708 najorningane mân-

na . . . 759 najoromarâtit : 617 okpertorotingit 148 pairivâtigut . . 539 piuliklerninga itt. 13 piuliklerninga

pik. . . . . 630 saimaningat . 269 sorrusiolune . . 663 tækkonârlise .593 tallinganut . . 786 timinga Gûb. . 640 timinga nerrivlugo . . . 844
Jêsub timinganik kunni. 128 timinganut . . 142 torkominut piu. 211 tunnergutauninga 270 tunnergutauninganut

Jêsu Krist! nulliarêng. 697 Jêsumit aritingilânga . 844 Jêsumat olpertinga. . 763 . Jêsus ajæktoratalo . . 449 aklunstsanut . . 92 ananaujôvlune . 191 anernangêrmat $\mathrm{K} .403$ anernangêrmat $u .146$ anernangêrmat $u .132$ Anernerpit . . 667 attorpakks . . 629 auktojut iklivit. 238 aulatsinermine .807 aungnut ubvaruk 603 erdligidlartara . 418 orraititigut . . 665 ijecartinga . . 221 illingnut tuksiarp. 399 inôgame tagra . 149 inurotitit tunnit. 253 . inurotivit . . . 438 ittersimajotit. . 747 ivlernartorsôjun. 646 rsilaurit manna $\mathbf{4 2 8}$ remmergoguplit 245 kênavit sângane 587 Eennuvigilugo . 263. kikkiaktorpât . 407 kikkiaktortaungm 116 kilalialaungmat . 164 Komuniônemut . 611 
Jêsus Kristuse Gûdib Ern.

Kujagivaptigit kil. 167 Kূujagivaptipit pi. 650 kutsaservigivavut 406 Kuviasûtiga . . 251 malliklugo . . 835

Nâlegatôjotit . . 605 Nâlegarut . . 369 nâpkijosuktotit . 454 napkigosungner. 551 nertornautingnut 688 pigekârnago . . 324 pituarijagit in. . 40 nitilaukpara ôm. 520 piuligangne . .870 Piulijiga! kap. . 259 Pinlijiga puigor. 838 Piulijivut kujag. 798 pivlunga tokко. 400 saimarsainit miss. 468 saimalaukittôK . 703 salutitaulerit! . 40 tarniptingnut sak. 249 tessiunga! inôtil. 339 tættigijara . .838 timingnik nerri. 624 tokxonernut ânia. 215 tokKungnut kej. 238 Jêsuse aiparigapko $\quad .157$ " aiparilutit . . 785 ajugaujivut . $531 \mathrm{a}$. âniadlartoK . 385 âniajotit . . .484 âniarnulle . . 413 âtautipâtit . . 113
Jêsuse attê mânna . . 757 auggit ikkitit m. 390 auggit ikkitit ôm. 112 aukpit kôktub 696 aulik erkitsom. 122 aungnut tamapta 490 aungnut uigas. 692 aut ivsornaitor 363 Betâniamit . 163 erkarangapkit . 519 erkilagit, illung. 91 Gûb saugârsunga 252 Gûb saugârsunga 815 Gûdib Erninga in. . . . 178 Gû̀dib Erninga ping . . . . 456 igvit tarnit an. 696 ikkajutsainartigut . . . 751 ikkilik! tuksiar. 384 ikkingnit illa . 411 illagêkluta . . 445 illagektitatit . 743 illagêktut. . . 447 inôsingnut . . 699 inukotingnut . 812 inûliklerte . . 812 ivlerijara . . 519 ivsorn. inung. $485 \mathrm{a}$. ivsorn. uving. $485 \mathrm{a}$. Kakкôjaujotit . 617 KanoK nâmaktomik . . . . 41 kattimarogut . 600 Kaujimavotit . 18 kejungme tokK. 372 
Jêsuse kênarnut illan. 552 Kenuêtotit . 485a. kiksarangama . 109 Krist najortigut 4 Kristuse Nâleg. . 690 makkisimangmat 157 mânna sângne 592 mânna toKkokit 648 mâne kissivit . 340 mânêpogut or. 603 mânêpogut tuss. 3 merngortornak $537 \mathrm{a}$. nagligilavut . . 426 nagligivagit . . 415 nagliktiga . . 768 nagliktivut ig. . 670 nagliktivut tam. 77 naglingnernut tus.

603 naglingnernut om. 704 naglingnernut on. . . . . 665 najudlartigut . 590 najulaungminga 225 nakorivagit . . 423 naksaungitomut 402 nakudlarpotit . 34 nakudlarpotit . 267 nakudlartotit . 40 nâlainartotit $485 \mathrm{a}$. Nâlegara . . . 763 Nâlegase . . . 569 nâmatuinartomik

$485 a$. nâpkiningnît . 357 nerrigangap. .780
Jêsuse okpertinga . . 540 ômativit ill.. . 81 pigiluarminga .400 pigâlauktotit $485 \mathrm{a}$. piloriktitiga . . 480 pituarigaptigit . 828 pitsiartotit . 485a. Piulijivut pissi 551 Piulijivut tuks. 93 pivlutale ik. .430 pivluta tok. . . 854 saimakit . . . 711 saimavok. . . 233 sângnut pâm. . 242 sulle nagl. . . 211 trekkolaukpotit 348 tækkonârupko . 232 taimak piginga 276 takpângat . . 774 tarniga nâpkigiuk . . . . 470 tarniga saim. . 468 tarningnut mânna . . . . 487 tokrotaujok. . 373 tokkungmat . . 249 tokrungnut aul. 739 tokkungnut na. 567 tuksiarpagit. . 478 tuksiarpaptigit 658 tunnigangne . 119 tussalaukpogut 664 tussalaungminga 487 tussartigut nel. 563 tussartigut tuk. 104 uigarnernik . . 705 uviningme . . 483 
Jêsusele ikkitit sulle . 635 illingnut tam. 513 ômarmingmat 155 sângne mânna 583 Jêsusemik perangitor 519 Jêsusemullo okpertor 267 Jêsusemut erkitaulertoK 422 Jêsusib ânianingita . . 86 âniaminut . . 496 âniangane in. . 140 ânianganut âk. 698 ânianganut kibl. 5̃14 âniangita piul. 541 Anerninga . . 313 attingane mân-

na... . 773 aunga ikkingillo 634 aunga kovijor 122 aunganelo ik-

king. . . . . 302 Gûb saugârsung. 99 ikkingit . . . 719 inôninganit saim. 316

Kennitsainarpâ 208 Kennuêninga . 355 Kennunikka . . 311 kidsijârninga . 622 Kristusib Gûd 806 naglingmattigut 90 naglingninga . 507 Nâlegapta kenn 190 nutarârsub . . 53 ômatiga naj. . 394 Piulijipse . . 882 sângane erkai. 141 sorrutsit . .667
Jêsusib tâpsoma mânna 160 tarnivut . . $814 \mathrm{~b}$. timinga aungalo 627 Jêsusible nellipsalaungip . . . 353 Jêsusiblo ikkinginit. . 247 Jêsusiga aggut, pits. . 224 âniagavit . . . 570 aut illûnât . 758 erkarupkit . 419 illagêt Nâleg. 589 illingnut mân. 873 issumaktut . $6 \overline{9} 9$ каiкogamga . 852 Kugviortiminga .. . . 470 Kujagivagit $\mathrm{m} .377$ naglingnernut 427 naglingnerpâng. 89 najornerne $\quad 853$
nertorlagit .358 okititsainangatôk . . . . 533 pairijiga . . 461 pêjarpattit . 238 piulinga! . . 488 pivlunga . . 117 sôngotikit . . 532 taimailerpat . 817 tâpkoale . . 601 tarniga . . . 841 tautukapkit . 84 tautukôrangapkit . . . 103 tokkovotit . . 427 ullapirsautingnik . . . 317 
Jêsusiga uvaptut pivl... 245 uvlometauk . 766 Jêsusigale tokkokârnak 646 illa kem. . 420 Jêsusima ikkingit . . 412 imâk oKau. . 271 inôtsinne tunn. 257 Kaujivait . .518 nâpkigivait . .214 Jêsusimale kollêne . . 99 Jêsusipta kiglinganit . . 37 " saimaninga 865 a. Jêsusîttauk . . . . 525 Jếsusivit ômatinga . . 209 Jêsusivut angerningit . 879

" nâlegarigap. . 701 Jêsus okarpoK • • • 24 Johannesetut illing. . 288

\section{面。}

Kaikojomangmattigut . Kaikogaptigut kailer . 669 Kaikogumga illingnut . 834 Kailaurit Jêsub akrane 181 " okumaiguvit. 214 Kailauritsetôk tæk. . . 144 Kairtor Jêsuse sapput 344 Kaitatit illûnatik . 305 a. Kajungidlalerpunga . . 831 Kajungertilauktigut. . 749 Kajungertitigêgitse . . 440 Kajungervigivaptigit $\quad .167$ Kâktut immeruktullo . 613 Kakugole . . . 158 b.
KammajomangitoKarpat . . . $172 \mathrm{e}$. Kammatsiaritse! Jêsub 471 Kangusukpogut nip. . 669 Kangusûtigijæksakka . 257 Kannimasivut âkikit . 611 Kannimat uvaptingnut 699 Kannitanganut âlaurap. 202 Kannivut angmartilugit 568 Kanoêleruma sap. . . .506 Kanoêmangâpselônêt . 211 Kanor ânianitit . . . 104 " Gûdib saugârsunga . . . 553

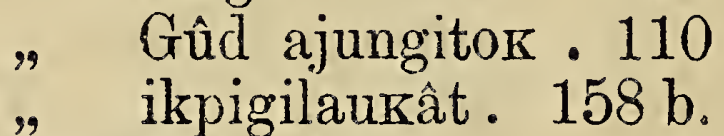
" ikpigilaukat. $158 \mathrm{~b}$. " illaliorlagit . . 53 " illingajuksauvât 682 " illingavise . . 861 $"$ issumavise . . 825

$"$ piniarlunga .282

" pivik Güdiga? . 158

"tagva sorrutsit. 665 Kanoktôk âniavit illa . 253 arvertarlanga 458 aumalerlanga 474 Gûb окаu . 602 idluitunut . 458 illûnâne . . 724 illunatik . $172 \mathrm{~d}$. illuvimnut . 134 inôtillunga . 140 inuit atunit . 422 inûlinartok $\quad 90$ Jêsub tamapta 504 Jêsusiga! nag. 482 Jêsusiga tam. 762 
Kanortôr raujimalerlit 504 nagligilago .417 nagliklagit . 416 nutaraunit . 53 nertornautingn. . . . 595 saimarsainit 357 tækkonaleruk 214 tamaita uvlut 417 tamapta . . 739 tautukôrlago 140 " uvagut . . 497 Kanoktôrle ânianitit . 379 nunamiut . 358 omativut . 706 piniarlanga 332

Kanoktôrlo ômatiga . 458 Kanorle Gûdiga . . . 770 pijuksauvîk . 30 taimaitor sul. 266 Kanorlo nâlektine . $158 \mathrm{~b}$. Kanorlônêt tessiorumga 308 Kappianadlartokut . . 345 Kappianamêk inuit . . 28 Kappiasumut nip. . . 842 Kappiasungner nuname 526 Kappiasungnermut na 231 Karâlit kakranginit 742 a. Kattaktitor auliglo Jê-

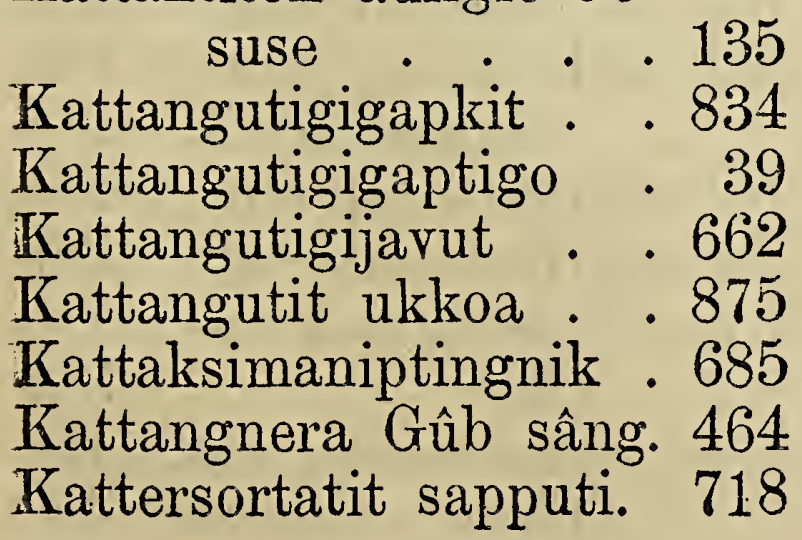

Kattijomârpatta . . 814 b. Kattimagapta erdling. 797 Kattimalerapse sumik 674 Kattimalerapta illag. . 575 Kaujerârnago ômat. . 202 Kaujimagalloarpotit .461 Kaujimajotôgavit . . 81 Kaujimavogut tamapta 350 Kaujimavunga Jêsuse nag. . . . 455 Kaujivunga Nâlegara . 251 Kaulerpat amalo • 790 Kaumajok Gûdib $172 \mathrm{e}$. Kaumajok Jêsuse! Gûd 227 Jêsuse . . .770 mânna sak. 859 nakudlartor 3 tâmna . . 49

Kaumajorsôjotille . . 50 Kaumajorle okausernut 198 Kaumajub tagva . . 265 Kaumaksalaungminga 228 Kaumaksartaujogulle 742 a. Kaumanekartilaunga . 573 Kaumanernut rauma. . 866 Kaumanerpit Kaumak. 276 Kaumartigivaptigit . . 811 Kaumartitsijok! каu. . 199 Kaumartitsinernut . . 877 Kaut tamât ômatimne 137 " tamât sapputitigut 484 " tamât serngnig. . 872 Keanartovingme mâne 855 Kejungme nipleravit . 211

$" \quad$ nivingavl. $\cdot 257$
$" \quad$ tokkogame 373
$" \quad$ tokkolauravit 334


Kejungmut kikkiaktortau. 239

Kellaklugillo avating. . 54 Kellaksorpânga Sâtanib 17 Kellaksorsimavunga . 838 Kellarudsâlaungmitigut 335 Kêmititsainartor illingnik . 289

Kemmaksinga idluitose 826 Kemmangnanga oKar. 519 Kemmangniængilâtigut 706 Kemmergosiuk Gûdivut 121 Kênanga Ketserarlugo 93 Kênavit kaumasingat $\cdot 291$ " tautuanut . . 124 Kênat illanârnartox . 429 Kennerialauraptigut . 376 Kennertaujut baptitau. 656 Kennertiga Jêsusiga . 503 Kennuêlune uttakкijoк 174 Kennuênermut saimar. 465 Kennuêrsârtotit saimanel.

258

Kennuniptingnut Jêsuse 754 Kennuvigivagit mânna $2 \varangle 1$ Kennuvig vaptigille .581 Kennuvigivaptigit Jêsuse 704 Kennuvogut Jêsus, aung. 363 illingnut . 748 Kerubillo Serafillo . . 814 KetserartaujoK kênang. 130 Kia pingortipase? . . 663 ". taimak pivâtit? . 110 Kiaptikarnît aullo . . 122 Kiægungnernut aunar. 358 Kialo ajorungnaitinivâse 674 KidsijârrtoK aungmik : 85
Kigligiudsijose 740 Kigligiutigilavut.$\quad .856$, Kiglisiniaruma uvam . 481. Kiglormulle ajornima . 109 Kikkaratik sinningilat 796: Kikkiaktortaugamelo . 121 Kikkiaktortaugavit . . 655 Kikkiaktortaunerminut 22 Kiksadlarnermut Gûdiga 223 Kiksarangama Jêsuse . 527 Kiksarnartovingmênapta 4 Kiksartitaukattartut . 856 Kiksartoksôlaukput . 162 Kiksartose ajornise . . 214 Kiksartut tamaita . . 705 Kiksarvingmêtillunga . 146: Kilait perkutigilugit . 46 Kilak nuna imarlo . . 171 Kilangmêkalloartotit . 163 Kilangme serrijomârput 167 Kilangmelo nunamelo . 154 Kilangmêpor illa . . 832 Kilangmêtotille Gûd . 190 Kilangmiogasaksuit . 42 a. Kilangmit avimajogut . 252 " nachoginata $42 \mathrm{~b}$. Kilangmullo nunamullo 814 Kilaublo ânanauninga . 606 Killamik najungikumga 326 saimanermik 218 . Killanârnera taimait. . 288 Killanâritsainaruptigit $\quad 289$ Kimut sânajartuksauvik?

Kina erkartorungnarkâ 154 " illiktut saimavâ . 449 
Kina tættigilârpigo . 845 „ piniarijane pirl. 264 Kinalo nagvârkâa $\cdot 264$ Kinaulaukpita sullelo . 509 Kingornertugivât _. . 160 Kingorlermik Gûb pine 522

" ikkivit . 114
" nagliktipta 116 " ômatigle 522 " nerrima. 604 tâmna illa 522

Kingorlerpaujomik . . 160 Kissarvingmik illa . 244 Kissiane mânna sulle . 363 Kissiat tagga kuvia. . 399 Kissivit Gûb Erninga $42 x$ Jêsusiga nagli. 431 Kuviagilagit . 304 nagliktara Jês. 715 Kittiksaungitut . . . 112 Kittorngarigamga, ajor. 224 Kittorngaritsianga . . 486 Kittorngârsuit Nâlegak 439 Kittorngaujose kaititse 440 KittorngauneK Kristusem

Kittorngaunerle Gûdem. $26: 3$ Kittorngauniptingnik G. 200 Kittorngavut illûnaita 697

$$
\begin{aligned}
& \text { piluarlugit } 702 \\
& \text { uvagullo } 695
\end{aligned}
$$

Kittorngat Jêsub ikkin-

Kittorngat Jesusemênaritse . . . .

Kittorngiolerupta . . 699 Kivgartorlutit Gûdiga 812
Kivgartorkolerangne . 870 Kivgartorkogaptigut . 717 Kivgartorkogumga . . 726 Kivgatit tamaita $.814 \mathrm{a}$. Kivgatun-ilauravit . .724 Kivgavit aggangitigut $172 \mathrm{f}$. Kivgaujungnaitilaungmi 878 Kivgaujungnaititaumaj. 683 Kivgauluta ajortomut . 23 Kivgavit nertoramitit . 722 Kivgaulerpor inukulluk 37 Kollarnermut okp. . . 212 Kollisse âkisigik. . . 824 Komuniônekartinga . . 285 Komuniôneme aitortor. 298 Komuniônît Hailige . 650 Koppergotuinauvunga . 836 Kotsingnerub kittorng. 503 Kriste, Gûb sauganga 118 Kristub aunga pigitugo

$531 \mathrm{a}$.

Kristum aunga idluartok . . . 267 inûlervia . . 52

Kristus inôngmat sôg. 149 ivsornaitôjotit . 23 Marîab Erninga 33 okautigênarlavut 2 Piulijivut . . 92 tættigivara . . 528 Kristuse Gûb Erninga, a. 133 " GubErninga! t. 92 nâpkigitigut . 865 nuname . . 16 saimarsainernut 488 Kristusemiut tamapse . 336 Kristusib piniarningit . 602 
Kugvileritik ijîkka . . 99 Kugvinut kujagivagit . 643 tautukpaptigit 145 Kugviorangapta mânna 289 Kugviorlunga illumne . 126 Kugviornermut mânna 374 Kugviortut-paingumut 158 a. Kugvioruma, mannigor. 195

Piulijiga .465
Kugvit allarterpait . .233 Kujagênaromavagit . . 112 Kujagidlarpara . . . 85 Kujagidlarlarut tâmna $54 \mathrm{a}$. Kujagijartorlugolo . . 803 Kujagivagit Jềsuse . . 413 " tagva . . 29 Kujagivavut Gûdivut . 236 Kujagivlugo Atâta . . 36 pingik. . 352 piuli. . . 674

Kujalimut nerrivlunga 319 Kujalinermut . . . . 325 Kujalita! Gûb aungat.. 405 Kujanak kennilaung. . 469

$\begin{array}{cc}\text { Kuvianak } & .858 \\ \text { nâgliktivut } & .388 \\ & .385\end{array}$

Kujanarle! inûleravit . 365 Kunnungipattalônêt $172 \mathrm{~d}$. Kuviagênarpagit как. . 429 Kuviagivagit nutarar . 44 Kuvianadlarmêk Nâleg. 543 Kuvianar allianamêk . 863 Jesub ikki. . 319 Kuvianamêk illa. . . 59 nelliutor 863 uvlome . 42 Kuvianarnerpâk unnuar 54
Kuvianartoliksoak . 54 a. Kuviasudlarpunga . 647 Kuviasugitse kilangme 862 Kuviasulârpunga . . 854 Kuviasulerit tarnîk Nâle. 268 tarnîk puig. 846 Kuviasuklunga nerriugi. 462 Kuviasukovlutit Gûde . 176 Kuviasungneralo ômat. 407 Kûviasûtigitsainarpagit 308 Kuviasûtigivât tagva . 576 Kuviasugvetuarut Jês. 707 Kuviasugviginga . . . 573 Kuviasugviksavut . . 704

\section{III.}

Magdalênatut-ilunga . 419 Magdlenaugalloarnanga 150 Magguk attausiojuk . 704 Makkilaungmat aner. . 154 Makkinime kingorngane 159 Mânna ajornerub tok. 31 " angmarmat ômat.214 " aularpogut tag. 741 ” aupta Kuttingita 377 " avatigigamga . .837 " erkarluarlavut . 350 " Jêsub timinganut 620 " kujagivaptigit . 653 " kuviasungnermut 808 " nagvârpunga ik. 246 " nagliktavut . . 567 " nagliktigêmut . 420 " okpervigigapkit 140 " ômatimne . . 353 
Mânna sulle . . . 158 a. " tagva nagling. . 423 " tikkilugo . . 550 " tikkisimajotit .. 46 tuksiarpaptigit . 599 Mânnale Piulijivut . . 583 Mânnamit inôsiptingne 697 Mâne inôtillunga . . 436 " ittiggangnêpunga 617 " kiksarviksavut . 79 " kugviorpaktut .838 " nachogijaugalloar. 536 " tarnit ajoKsartor 49 Mầnepunga Gûdiga . . 393 Mânêtogut perkojatit : 729 Mannigoijôgavillo . . 198 Mannigoijotit-kiksar . 156 Mannigolaungmingale . 29 Mannigornadlartor tag-

$\mathrm{va} . . .402$ Mannigornarpok ôma . 259 Mannigortauvogut Jês. 689 Marîab Magdalênable . 157 Marîatut ittiggangnut . 15 Mattoma kugviortip. . 263 Mattomane illagêk . . 606 Mattomanelo uvlut ta. 587 Mattomanêtillungale . 326 Mattomanêtsungnaipotit 197 Merngoêromaguvit . . 272 Merngoêrserle sinnikle 138 Merngoêrsertuksaumang. 715 Merngoêrservigilavut . 708 Merngoêrservingnullo . 134 Merngoêrsidlarpunga . 293 Merngortortok timiga . 79 Merngortorpalaung . . 663
Miksekârnerub tussarnidl.

Missigalloarpungalo .511 Missiginarmêk . $158 \mathrm{~b}$. Mittautigijaudlarner . 842

\section{N.}

Nagligijomavagit. . . 841 Nagligiluarmattigut : . 214 Nagligivingârvigivâtigut

158 a.

Nagligosuktub Jêsusib 22 Naglikapse illa . . 165 Naglikattigêtsiartut . . 443 Naglikparma issuanit . 427 Nagliktakka piniarta . 15 ," tâmna . .663 Nagliktara Jêsusiga . 196 Jêsusiga kuv. 818 Nagliktavut Nâlegavut 80 Nagliktiga Jêsusiga - 51 " $\quad$ tagnna .479 Nagliktigalo Jêsuse . 32 Nagliktigêgitse perk. . 444 NagliktoK Atâtavut 183 " Jêsuse! igvit 882 " Jêsuse puig. 645 " Jêsuse! saim. 240 " Jêsuse sumik 102 " Jêsuse tikki. 127 " Nâlegak tam. 336 Nagliktotit uvaptingne 548 Naglimut amualauкp. . 246 
Naglimut Kellaksolauk 358 " nellekangitomut . . 365

" pingortip. 658 Naglingneк angnerpâk! 628 kajungner. 488 $" \quad$ kajungner. 488 " nâpkining. 28 " perkojiguvit 440 Naglingnекartimitigut . 445 Naglingnerle taimna . 446 Naglingnernut illuvut . 447 Naglingnersarijomavagit . . . . 333 Naglingniktôjok! tagva 374 Naglingniktor Jêsuse . 69 Kennu. . 752 Nâleg. . 781 Nâleg. . 691 Piulijiga 480 uvapt. . 553

Naglingniktôgame . . 593 Naglingniktotôgavit. . 765 Naglingninga angijok! . 465 $" \quad$ angivok . 518 Naglingningane merngo 730 Naglingninganut aumav. 244 Naglingningat erkaitipa Naglingningnît innornar. 604 " pitsartu. 604 Naglingniojotit igvit . 348 ômat . 199 " pitit 814 a. Naglingnît illitarivara . 511 ipaksinangi. 658 Nâlegara . 229 uvlut unnuat 94
Naglingniub akkartipa 488 Naglingniub nellekangitub . . . 244

" . pêjarpâ . 433 Naglingnivit kissime .30 Najoromadlarpaptigit . 124 Najortigut Anernernut. 789 " Jêsusiga . . 568 Najudlarangne kiksar. . 543 Najuênalaungmitigut . 546 Najuênaromavagit . . 515 Najuleraptigut Kuvia. . 397 Najutsainarmitigut . . 750 Najutsainartigut illa . 716 Nakoridlartaule illa Jês. 150 Nakorijaulit Jêsub ik. . 641 Nâksaungitomut nag. . 279 Nakudlarpok Gûdima . 744

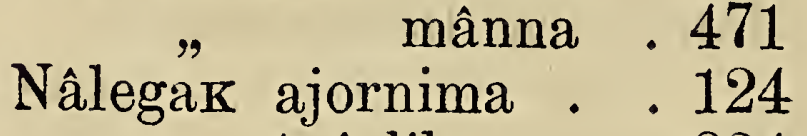
» ânialik . . . 834 " angijôvok pin. 172 " âniavit . . . 114 " attaviksak .535 " avitsangilak . 803 " Gûd Anernerp. 547 , Gûde Anerner 198 " Gûde nagliktot. 78 " Gûdivut! pig. 466 " ikkajortigut illit. . . . 691 ikkajortigut ka. 443 ikkajunga . . 773 illûnatik . . 854 illuvut . . 809 Imanuêl . $731 \mathrm{a}$. inôsekartiuk t. 485 
Nâlegar inôseкartiuk . 485 inôsekartinga 485 inuit tamaita 210 ivsornartunga 21 Jêsu, saim. . 753 Jêsus kissivit 4 Jêsus Kristuse! 599 Jêsus nangmiпек . . . . 590 Jêsus nertorlavut . . . 813 Jêsus oxausernut . . . 12 Jêsuse illagit 778 Jêsuse illingnêl. . . . . 281 Jêsuse illunata 591 Jêsuse pigingik 231 Jêsuse tættigi 775 Jêsuse! inôg. . 277 kattersoigavit 588 Kaujitilaunga 453 Kennuvagit . 524 Kristuse! nak. 165 Kristuse! tagva 645 кujagilavut . 153 mannilaurit . 467 nagligijavut . 286 nakorilavut .808 nakorijaulaurit 406 nakorivlutit . 796 nakudlarpotit 267 nâlengnartok . 802 nâletsainarpâtille. . . . 587 nâpkigosungnît 247
Nâlegak nertorpagit . 311 nertoruk mânna . . . 802 ômamut kig. . 235 opigidlarpât . 722 opigivagit . . 557 opigosumut .737 pairijigivara . 192 perkogaptigut 512 pidlarnangatôK 226 pijarêrtitsivok 166 pingortipât . 362 pitakaloringning. . . . 725 pivlunga . 14. sângne tuksi. 288 taimak pivl. . 596 tarnivut . . 751 tokkoler . . 263 tunnijarnik . 459 ussornaravit . 375 Nâlegapsingnut sâtitse 546 Nâlegapta tâmnale . . 264 Nâlegaptalo sângnut . 698 Nâlegaptingnut âniaj. . 202 Nâlegara Jêsuse Krist! 842 Jêsusiga . . 148 nagliktara! · 714 najulaunga .419 orautsitit . 7 saimaunga! $\cdot 731$ Nâlegarle! angerpogut 546 Jêsusiga! . 326 najudlartigut 562 nulliarêk .694 okperpunga 238

Nâlegatuara Gûde . . 572 
Nâlegaujok illagênut . 586 Nâlegaunit kailaule! . 190 Nalegaviksôjotille! . . 581 Nâlegaviksûb Gûd . . 44 Nâlegavut ikkajortigut 570 ipaksinang. . 752 rêlerame .116 mânna Kuja. 73 nagliklavut . 437 nakorivavut 566 nertorlavut . 804 Nâlegitse illûnase . . 714 Nâlekab aulatsijotô-

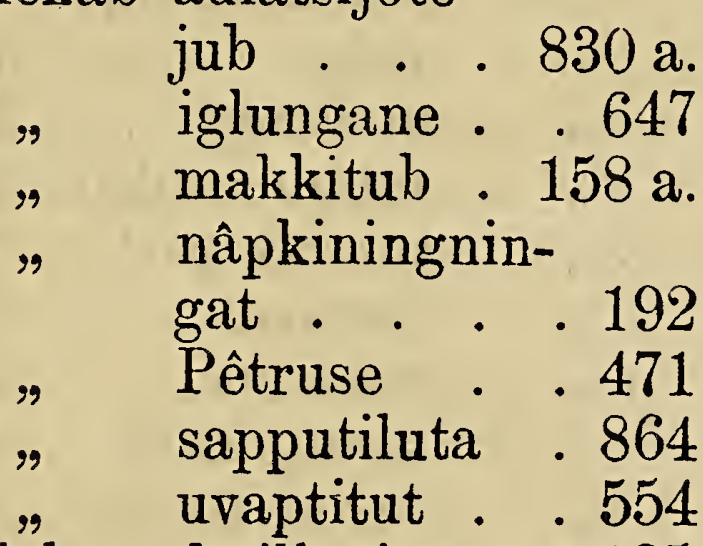

Nâlekamalo illuvinganut 135 Nâlekama toкKovine . 125 Nâlekamut ailerta . . 77 Nâletsiartomik . 172 a. Nâmagijanganut . . . 479 Nâmaksidlarpunga pigi. 131 Nâmaksivor . . 234 a. Namut Kemâjuksauvík? 222 sâniarpik? . . 224 Nane Gûdeкarka illiktut 210 Nanelônêt-ikuptale . . 143 angmineK kennerang-

ne . . . .876

Nâpkigællutalo saimavor . . . . 289 Nâpkigijaukârata . . 254
Nâpkigijaunine erkaumat.

Nâpkigijauvigivavut . 586 Nâpkigijaksaungitunga 239 Nâpkigosuklutit illa . 505 Nâpkigosuktôgavit . . 670 Nâpkiniktôgavit . . . 224 NâpkiniktoKsôgavit . 710 NâpkiniktoKsônîllo . . 253 Nâpkiningat angijoksôning. . . . 509 Nâpkiningnernut Jêsus 866 Nâpkiningnermut piul. 24 Napkiningnît angidlarp. 363 Jêsus .619

Nautsertutsiarsiuk . . 110 Nekkorsidlalauraptigut 651 Nekkorsijaukovluta . 608 NekkorsinaK nutak. . 169 Nekkorsinarput illa . 132 Nekkorsinartotôjor $\quad .860$ Nekkorsinartor Kom. 626 Nekkorsitilaunga atti. 219 Nekkorsitipâtigut timing. . . . . 608 Nellâgôrlune tâpsoma 449 Nellâgut illumne ‘. . 479 " ivsornaitomik 23 ômanartomik 616 Nellautaijunullo pivok. 23 Nellekangitôjor nagling. 347 Nellekangitok ânianga 54 Nellerangitor ômat. . 704 Nellekangitotit Gûd. . 476 Nellekangitub tâpsoma 612 Nelliukârtinnago Jêsub 608 ang. 401 
Nelliutijok serrinak . 328 Nelliutitârpor illa . 817 Nelliutingmat, Atâtamit 54 Nelliutor Kuvianar! . 35 " serrinartok . 65 Nellojungnaitilaungmit 681 Nellojut pinlijingat . . 25 Nellonarpor ranga. . 844 Nelloprotidlarupta Kuvi. 327 Nellopкotimut kaititse 359 Nellopkotivunga tagva 469 Nellunaikutarissiuk . . 32 Nênertorungnailerpânga 45 Nênertaujutiksaptingnit 232 Nerchevingme nanger-

pung. . . . 47

Nergutit nercheviænne. 45 Nerkigivavut orautsitit 167 Nerkiksax pijariak. $\quad 779$ Nerriugidlartamingnik $42 \mathrm{c}$. Nerriungninginit piluar. 711 Nerriugviptingne tamaine.

Nerriuktor okperidlar. 744 Nertordlerutivut . . $814 \mathrm{a}$. Nertorlagit Jêsusiga! . 766 "nâleklagit . 11 Nertornar tamainit . 298 Gûd ajortuggit . . 154 Nertornarnît Gûdivut 866 Nertornartor illa . . 822 Nertornautiksarnulle • 184 Nertortaulaurit . . . 148 Nertorungnangikupkil. 375 Niarox, Gûd pairiji. . 261 Niakor ikkilênar . . 124
Niakôsertaujorlo kak. 410 Niakôsidlartaunîllo • 239 Nipliangmat Gûdiga . 92 Nipperaruvit . . $172 \mathrm{~h}$. Nippingajokaranetôk . 338 Nippît illume tuss. . 203 Nipsausijartut Engelit 823 Nivingajorlo kejungme 125 Nukapiangoniptigut .678 Nukapitsat illûnase . 678 Nulliarêk ukkoa . . 693 Nulliarêngniptigulle . 697 Nulliarêngnivut mânna 695 Nulliarêngortitsijôgavit 700 Nuname illagêngnivut 586 " inôtilluta . 807 " pijokartune 172 e. " sorrutsine . 843 Nunamêkojomagumga 666 Nunamêninganelo . . 870 Nunamêniptingne issu. 686 Nunamêtillunga, Jêsuse 332 Nunamêtillungale . • 158 Nunamêtilluta Jêsub $\quad 369$ Nunamik kemailâroma 848 Nunamut kovivât aung. 17 Nunanut illunainat 742 a. Nutakкatut keajotut 427 $\begin{array}{lll}\text { \# pitiminga } & 11 \\ \text { ilertitigut } & 289\end{array}$ Nutâmik attitilauruk 869 " illa inôtsivut 727 Nutângortitigut Jêsus 227 Nutarar Jêsuseasuk . 74 " nakorivagit . 57 , tattamnartotit 51 Nutarârsuk erdlingn. 51 a. 
Nutarârsuk Jêsus! · · 42 Jêsusiga! 40 Jêsusivut $42 \mathrm{~b}$. ussornartok 38 Nutaraunerminillônêt . 261 Nutarauniptingne bapt. 667

\section{(1.}

OKaulaurit Nâlegara • 11 OKarnæk: sappernart . 214 Orarniarminak : sulle . 214 Oкаrpotit: immeroma . 432 Oкаrtокагрок: ajortut 208 OKaupânga ungaginga. 17 Orausek Gûd nâkssaungi. 28 OKauserlo kingorlerpâk 140 Okausernik ômatiga . 11 OKausiksæropogut sâng. ne ... . 360 Окаusingalo okperigapko . . . .

Okautaujungnangimat . 577 OKautaujungnatsangilak 829 OKautigijæksarivavut . 658 Okautigijomagupsiuk · 728 Oкautigilavut tagva . 385 Oкautigilerpagit ovanêt. 374 OKautsikka malliktârkit 17 Okautsille tоккоtaunernik .... . 12 Orautsillo imaitut . 79 Okautsit Jêsub ikkinginnik . . . . 576 Okautsit pêrungnangi-. lait
OKautsit tussarnerpât . 660 tussarnertut . 60

Окаutsitit illa ајок. Piulijîk . . 866

OkoKse! Jesus pitsiarlu 430 Okperaptale okarpogut 640 Okperlune naglingniktor .

Okperlunga illiktut. . 248 " tarnekut . 401 Okpernartôjotit . . . 468 Okpernek Jêsumut . . 500 Okpernermik illumne . 248 illunata . 652 Okpernermut aivigivagit 240 Jesusemut 236 Okpernerub kaumasinga 296 saimarsarp. 23 ; Okpernikitub tarnima .276 Okpernima kissarviksane 244 Okperpunga Gûb Erning. 267 Jêsub aung. 267 Okpertokotinne innigi . 543 Okpertose inôluaritse . 530 \#. tamapse . 41 Okpertulle Kristusemut 28 nunamêtut . 206 Okpertunut imâk . 165 Okpertut ajugautitse 531 a.

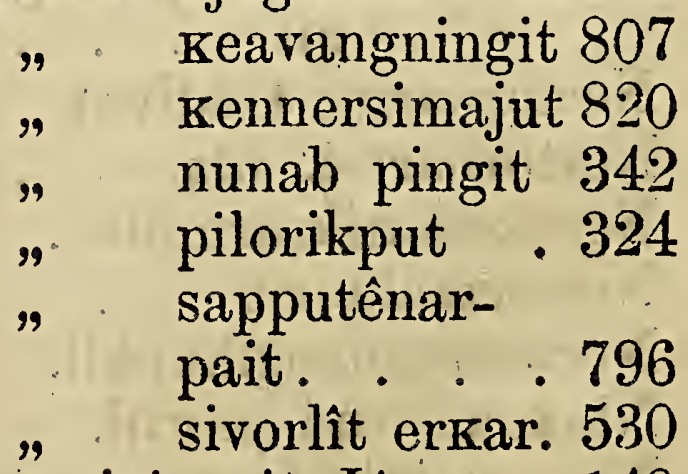
529 Okpervigivagit Jêsus . 149 


\section{Nippisiorutit.}

Okpervigimarikpagit . 845 Okpingitut akkorngænne 597 " ômatiga . . 109 Ôktortaulungnartomut . 190 Ôktortauvingmêtillunga 527 Окитаitokut pijuksau. 337 Orumaijutitit Gûmut . 500 Ôma naglingningane . 593 Omat aggait pitipavut 652 auktojor pivlunga 643 ajortolik kap. . 397 kappiasuktor . 141 nertornadlartoK . 867 sôgle sillaidlarkit? 28 taktuglo igvit . 696 Ômateraitigekluta . . 440 Ômatib ablatsauningat 691 Ômatiga ajoksartor . 173 assingnut . . 275 aumavor . . 104 kealerpat . . 496 keagangat. . 47 pannertotun-ip. 629 pimariguk . . 285 pijomagangne 275 serngnigiuk . 666 sittijoK . . 222 sôg kiksarkit 173 tækkkotsainarle 287 tagga Kaipara 280 tamaitalo . . 274 tarnigalo .285 Ômătigalo illûnât . 666 Ômatîgle mânna . . . 228 Ômatîk angmalerille . 94 ullernairuktôk 32 Ômatille tættædlarmat 651
Omatima illâle . . 104 Ômatimne atternik . . 841 " erkaumaler 325 " tagva Kil. . 141 " tagvane . . 124 , tokkonit. . 134 Ômatimnêtsainarlit . . 481 Omatimnik kaitsivunga 276 Ômatimnut Kanim . . 395 Ômatinga angmarpoк . 525 tættædlarmat 706 Ômatingit kellaksokit 879 Ômatipkulle Gûdiga . 21 " takordligam.

265

Ômatipkut aumadlar-

lunga . . . 95 Ômatiplkut erkilaulagit 240 " mannigorp . 400 Omatipta tungavinget . 4 Ômatiptingnut itterit. . 689

" mânna. 405
nêrnertol.

626 Ômatit angmainarle . .638 " angmaruk mânna617 " kappiasukpat . 259 " kugviorningit . 705 " issumallo . . 644 Ômativit illagivâtigut . 54 Ômativullo ânianganut 202 kajungilâd. 614 Ômativut aungnut idl. 794 aungnik im. 432 erkartokit ... 194 issuanut . . 488 mannigokit . 705 
Passijaksaungitotit . . 105

Pattangajose tamapse . 45

Pêjaivigijungnarparma . 226

Pâjarlugillo aungnut . 278

Peкangarviksak . . . 808

Perkutigidlarpaptigut . 343

Perкutigijomavara . . 232

Perajaluktut tamaita . 756

Perkojanga uvaptingnut 271

Perkojarnik tamainik . 688

Perkojatit malliklugit . 666

Perkutaujose Gûdemut 148

Perkutaulangaletôk • . 629

Perkutaumarikkupta . 433

Perkutautsainarlanga . 10

Perorsêvingme keanivît 90

Perorsêvingmêdlutit . 88

Perortok sillatuvlune . 678

Pigannerpalliagupta . 612

Pigannertilaungminga . 229

Pigâritse pissiluse . .820

Pigârtut kotsiktomêtut 823

Pigigapkit Jêsusiga . 157

Pigijarnik sapkutsinak 338

Pigivarma Amenêrv. 283 a.

Pijariakarnerpâk • 273 Pijautitsivotit. . $\cdot 172$ a.

Pijomajarnulle . . .628

Pijominadlarpok. . .677

Pijominak кakugo . . 833

Pikkalo idluitut . . . 428

Piksakartitsainartigut . 186

Pilatusib Nâlegara. . 97

Piloridlarput kapp. . . 543

Pilorikpor inuk tâmna 819

" illa ullapirs. 301

” $\quad$ kollerminik 819
Piloriktoкsôvor illa . 168

Piloringnarmêk illa . 330

Piloringnek angivok .637

Piloringniksat illûnatik 334

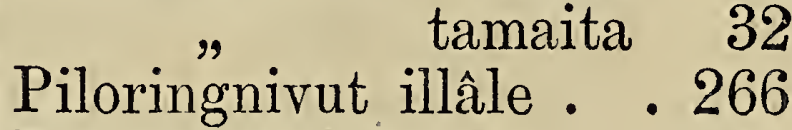

Piluarpâtit Dâvidib . • 46

Pinasualernera . . 175

Pinele remmakârnagit 652

Pingajuænnik illaminik 116

Pingasojuanik окаrрок 116

PingiiaineK pêrlugo . 382

Pingimallo angumit . 25

Pingmat pidlaræksau-

nera . . . 21

Pingmijor: nâpkigilak. 23

Pingortilauktiga . . . 572

Pingortitarnik tamainik 194

Pingortitigale . . . 128

Pingortitsigaville . . 53

Pingortitsijok tamainik 32

Pingortitsijub tamainik 28

Piniarnek ussornartok 610

Piniarnersoatit . . $172 \mathrm{~d}$.

Piniarningille . . . 298

Piniarnivut nuname • 45

" okautsivut 632

Piniaromagune . . . 507

Pinnarnadlartotôjoк . 410

Pinnarnartok nutarak . 69

Pinnarnartotôjotit . . 403

Pinnarnartotuak Jês. . 127

Pissiarigamga . . .606

Pissiarigangne tarniga 142

Pitiminga Jêsusiga . . 671

Pitipât Jêsusiga . . . 387

Pititaugame unnuar .609 
Pititaungmat unnuame 737 Pitlarapko erkaivunga 101 Pitlaraksatôvunga . . 110 Pitlaraksaunek pêrmago 22 Pitlaraksaunermik . . 450 Pitlartaujutiksakka . . 110 Pitsartudlartorlo. . . 72 Pitsartudlartut . . . 797 Pitsartunît Jêsuse . . 870 Pitsartunivut sungilak. 529 Pitsartutigivagillo . 196 Pitsiarnermullo . . $172 \mathrm{c}$. Pitsiarnerpaujor . . . 473 Pituarigapkit Gûde . .508 Piuligamga torkungnut 519 Piulijaujose tamapse . 365 Piulijaumajose tâmna . 234 Piulijaumajosele Jês. . 439 Piulijautigivavut. . . 39 Piulijauvogut illa . . 166 Piulijiga ajortunga . .673 âniadlartok . 106 atílaukpara . 844 aulik . . .633 idluilutakka . 278 illa kibl. . . 110 Kennuvigivagit 661 najorlugo . . 767 najuênanga . 265 najunga . . 192 pâmakpunga . 230 piginga. . 71 pivlunga âni. 107 pivlunga sor. 666 pivluta kik. . 616 tokkotigigam. 265 tuksiadlarpagit 733
Piulijigênarlagit . . . 45 Piulijiksakalaungilak . 365 Piulijiksaungitunik . . 234 Piulijîk inûlinga. . . 112 , takkonâlaungm. 474 Piulijima Jêsusib . . 643 Piulijipta Anerninga . 201

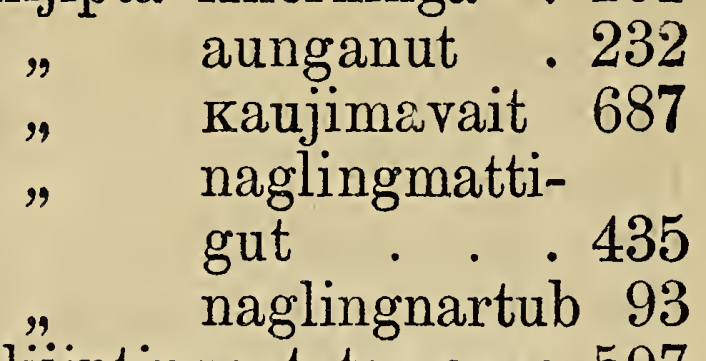

Piulijiptingnut tamapse 597 Piulijisele капок . .674 Piulijivut aulik . . .875

" ikkajortigut . 764

" illagêkititavît 624 " Jêsuse er. . 36 " mânnakut . 571 " saimarsaijung. 287 , tamapta . . 734 Piuliklernerniglo tamainik . . . . 692 Piuliklernernik tamainik 289 Piuliklernîllo najornîllo 292 Piuliklerteokovlugo . $\quad 28$ Piuliklertor taimak . 24 Piulilaungikuptigut . . 299 Piulimajose imgitsiaritse

814 a. mânna . . 256

Piulivlunga tikkinnavit 67 Piulivluta tokkovok . 840 Pivalliakôrpallo . . 537a. Pivianadlarmêk . . . 268 Pivianartor Jêsus . . 429 Piviksanga nelliungmat 23 
Piviksara tikkilerpat . 837 Piviojuksaudlarmat . . 401 Pivlugo mânna Gûdemut . .
Pivlunga inungorsim. \# Jêsuse tokkot. 250 " Jêsusiga kik. 428 " kappiasulaurame . . 131 " $\quad$ Kenuvagit. . 731 " kikkiaktort . 131 " taimak tiglukt. 131 " tokrogame $158 \mathrm{~b}$. " tokKogavit . 382 Pivluta inôlauravit . . 583 paungardlune $158 \mathrm{~b}$. tokkogavit tuk. 391 tokKolauravit . 57

Pivlutit ivsornaipunga. 45 Korlorpok . . 257 Pivor imâk Ernerminut 17 Puailavît tellimauvut . 123 Puigortaililago . . 283 a.

\section{.}

Sannikterijok Jêsusemut 264 , ômame 266 Sannikterivunga nagl. 228 Sâgiarangatta tammars. 264 Saimanartor pilauktara 255 Saimanartut aggait . 127 Saimaneruulle kennilau. 579 Saimanerub angininga 323 " anerninga 285 " sakkijârtub 439
Saimarlugit tarnivut . 748 Saimanartovingme mâne 490 Saimaneк ânanauvor 254 " angidlarpor 254 " nuisimavor 149 Saimanermik nagvâr. . 439 ullapirs. $\quad 556$ Saimanernêtsomavogut 571 Saimanernut Jêsuse . 685

, Nâlegar 866

Saimanerub kôtsainartub 514 Saimaningat angininga 257 Saimarsaijôgavit tuks. 619 Saimarsaijotit Jêsuse! 194 Saimarsaijuksakatsængim. . . . 2 Saimartigut Jêsuse! . 563 Saimartigut Piulijivut 489 Saimartimmagillo . 160 Sakkervigilaungminga 124 SakKervigiluta soraingi. 536 Sakrerviksat nelliutik 849 Sâlagijaukonnata . 537 a. Sâlakarviksioravit . . 163 Salutitauvotit Jêsusiga 156 Sânganêtose Saugârsub 801 Sangêtut pairidlarangne 460 Sângne Kakkialerpunga 276 , okausiksakarung. 629 Sângnut Jêsuse pâmakp. 511 Jêsuse pâmakt. 629 Sapputsauvigigaptigit 194 Sapputilauruk tarniga 427 Sâtanit illûnatik .176 Saugârsub aunganut . 250 Saugârsugle tâmnauvotit 19 
Saugârsugle torkotitar 260 Saugârsuk Gûd illagên. 366 Gûd tunnilaur. . . 501 Gûdeviojor 94 Gûdivut, tor. 565 idluarsinarto $536^{\circ}$ ikkilik illa. 444 ikkilik lkivg. 720 ikkilik pivl. 371 ikkiliksoar. 402 Jêsuse! ivs. 96 Immanuêl 549 rejungme 310 naglinguer. 405 nangmaleopinax! $\because 479$ opinarporilla 379 pilauruk kej. 96 pivianadlart. 453 salaralaurt. 335 saimanernut 393 tâmna tarnip. 816 tunnidlalauk 741 Saukanik pairrsijomit 613 Sâvigivagille mâñna . 258 Serkerngub mikserâritub 53 " nuilertub . 50 Sexkinek nippilermat . 92 nippilermat . 795 Sekxiniodlartotit Nâleg. 229 Sennerkab kappininganut 842 Sennerkakut kapping. 487 Senneranganêtsainarnerm. 400
Senningajolingmêdlutit 452 Senningnut aijomavung. 846 Sêrkortorvigivâtit mâne 576 Serrijutigivara siark. 412 Serrille tarne . . . 859 Serriluta kujalimut . 363 Serrinartut tikkiupait 39 Serrinarvit nelliuningit 174 Serringmiguma . . 195 Sillab pingit allarlugit 276 " pinnarnarninga 170 Sillaidlariunga! tautungi. : • • . 265 Sillainivut ikpigivarut 201 Sillarsoab illusingit . 328 Sillarsoakarningane . 261 Sillaksoarmiulle kattadl. 362 Sillazsûb pingortitinga 34 Sillatujotuak . . $814 \mathrm{~b}$. Sillatunima . . . . 221 Sillatunivut illa . . 3 Simmêjub akkilerpait . 151 Sinnaungajungnailutit . 169 Sinnigomalerama kauj. 793 Sinnigomaleruma pig. 389 Sinniktilauktigut sap. 786 Sinnilerkârnanga $\cdot .786$ Sinnikuma pairivânga 185 Sinnilerupta erkaumalap. . . . . 794 Siorniorner nuname .526 Sittamanganik niplerpor 116 Siumut tussartaungilak 159 Siutivut angmakit . .748 Sivorlermik Atâtane . 116 Sivorlilaurit, Jêsuse igvit . . . 337 


\section{Nippisiorutit.}

Sivorlipta uttakrivât . 29 Sivorliovor tamainit . 168 Sôgle angijomik pitlalauk . . . 102 Sokrosijuitikit Jêsuse 703 Soraijuitomik mânna 149 missigijæk 574 Sorainægle Gûde . . 500 Sorainanga erkaumaj. 744 Sorainangale tuksia- rapko 265
Sorlo arnab nuname $\cdot 77$ " merngortortor . 139 " nuname ômajut 237 " okaravit immerti. 432 " pingortigangne $172 \mathrm{~d}$. " piniaravit . . 114 " Piulijiminut Mar. 329 " sorsuktuksab̉ mân.125 " unnuar . . .54 a. Sorlolo kilangmiut . . 190 Sorruserle Jêsuse . . 53 " Jêsuse tamm. 665 Sorrusiogalloartunga . 671 Sorrusiogama mânna 666 Sorrusiolauravit ôm. . 675 Sorrutsinik naglingnikt. 881 Sorrutsit kiale . . .674 " perrutitit. 675 Sorsuktut annoraijarpât 737 Suksaungigalloartunga . 629 Suksauvâk tamakkoa . 663 Sulle akkunera mâne . 705

" Kaujerkârtinnanga 666

" merngoerserv. 833 a. " nunamêpogut. .861 " nunamêtillungale 511
Sulle piojorigama . 508 Sullinimnik nellovunga 257 Sumigle akkiksarmik • 88 Sumik okalerlanga . . 736 Suna Kuviasugvigivitigo 264 Sunamullônêt nuname . 269 Sunamut assianut . 420 Sunaungitôlungilatit $172 \mathrm{f}$. Sunaungitunik • . 830 a. Sungiutiner illingnik . 419

\section{塩。}

Tækkolaulanga . . 134 Tækkogama suilamnit . 257 Tækkokârnago okpervi. 614 TækkoKarnagole Jêsus 565 Tækkonânga nagl. . . 390 " pingort . 456 Tækkonârninganut . . 386 Tækkoranernerpâk . . 398 Tæklkosarner ikkingnik 412 Tagga Engele - . .42 a. " illa ômatiga. . 812. Tagva aggangnut . 836 " erkaumadlarlavut 70 " Gûb ânianganik 116 " Gûdib Erninga . 608 " Gûd tæuttigivara 223 " illa tattaminiarp. 102 " illûnatik . . . 243 " Jêsub Kanim. .614 " Jêsus nagliktara 485 a.

" Kaititse tamapse 45 " kiksarnermut . 516 
Tagva kuviasudlarpogut 289 nagliktiga . 284 nâlegara . . . 479 nanêkuma . . 298 nellonangitomik 358 nutarârsuit . . 843 okpernermut. . 628 Paulus avitsomaVOK . . . 830 a. ômatiga . . . 85 opigosumut . . 205 ovanêpunga . . 136 pivlaurittốK . . 89 piulingmase . . 663 serrivogut . 827 soraernanga . . 128 tækkolerpunga . 132 tamât pivâtigut 34 tautuktiparma . 259 tarne anern. . . 261 time tarnelo. . 608 timivut tarnivut 682 Tagvainar toккоlauк. . 263 Tagvale erkaivunga . 88 " inôtillunga . . 419 " Kêmitikpogut . 612 " nertutsainarom.220 " sannikterineк . 263 Tagvane Gûdib kivgane

531 a.

кemergojoma 135 Tagvanele illanârijiga . 144 Tagvanêtsomalauktunga 140 Tagvanêtsomarungale .126 Taiksaungitomik . . . 472 Taimâgle inôtillunga . 267 $\Rightarrow \quad$ itikkatit . .587
Taimâglo idluarivat . 32 tarnit timillo 687 Taimaidlarmat . . . 857 Taimaigalloartogut . . 459 Taimailerupta . . . 263 Taimailiorkovlugit . . 703 Taimailungipat . . 8 Taimaimalle ikkingnut 534 opigomut 58
tunnidlarp. 201 Taimaimat avatitit . $\quad 89$ inôtilluta .602 issokangitor 813 Jêsub aungat 481 Jêsuse nek. 432 Kennuvogut 603 neriukpunga 47 nertorpaptigit . . . 796 ômat nelleк 131 ômativut . 360 piulijivut . 526 sorrusiarsuit 668 tamapta . 16 tâpsoma au. 267 tarnîk mânna . . 175 tættigileruk 807 tillijatittauk 738 tunnidlarp. 705 tussumarik. 742

Taimaimattauk illingnil 763 Taimaipat time . . 820 Taimaitoкangilak . . 264 TaimaitsainarKovlunga 508 Taimaitsainaromarivor 366 Taimaitub nellâgut . . 708 
Taimaitut illagênarpait 687 Taimak ajortullinimnut 99 âniangmat kin. 674 . âniatitaungmat 737 asserorsimaneK 23 Gûdiga nekoк. 195 idluarsinartông. 2 illa pairigumga 196 illa Piulijise . 598 illagivlutit . . 81 illapsugijane . 261 illioruvit . . 259 Jêsuse illagêt 442 Jêsuse nagl. . 435 Jêsub aunganit 382 kamagilaungm. 305 kannimakôrtut $47^{\prime}$ : kissiane nel. . 574 kissiane pin. . 500 Kuviasukpogut 33 nellop кotijunga 496 sâlakalaurivut531a. sulliarijane. . 755 tagva amulaunga. . . . 499 tautukôrapkit. 88 uvlok nâlavut 765 Taimaktauk angajokâvut 664 $\begin{array}{lll}\text { \# } \quad \text { Jêsusiga } & .158 \\ \text { kippiler } & .329\end{array}$ naglikap. . 664 Nâlegapta . 77 nellungilavut 77 pullasâr. . 237 uvanga . $172 \mathrm{c}$.

Taimna kikiaktort. . . 155 Taipkoa kaumajôvlutik 796
Taipkoale kippilertut . 23 Taipsoma illuane . . 23 Takrakka tiglertillugit 785 Takrerlo tækkogapko $\quad 170$ Takupsoaginerpângojox 473 Takpâne attatitsemik 861

" kilangme . . 243

" Kilangmêtsarêrрок . . . 830

" ômat ivsornaitor . . . . 331 Takpaunga ârlorpunga 91 TâktoKsoarmêtillunga $\quad 47$ Tamainele Jârine • . 79 Tamainik pingortitsijor ak. . . . 737 pingortitsiјок G. . . 57 pingortitsiјок n. . 33 pingortitsijotit : . . 742 ajortunit . . 190 piksak Jêsuse 322

Tamainut Kuvianartotit 46 " sarkeromârm. 31 Tamaita ajornivut

piting. . . 663 ajornivut Piul. 477 ipperarpakka 418 Kattangutikka 852 piniarnivut . 699 uvlut . . . 409 Tamakkoa erkarlugit 266 illûnatik . 239 Tamäkkoale illumne . 424 Tamanêkojomagamga 322 
Tamanemiôtillunga . . 842 Tamânêpor Atâtab . . 27 Tamângat aulalerapta 784 Tamanna attorangaptigo 646 " Engelit aglât 74 " erraraptigo 682 " pivlugo illa 233 " pivlugo mân. 224 " pivlugo nagl.441 " tattamnadlarрок . . . 261 Testamentin. 652 Tamannale pivlugo . .114 Tamapse Nâlegaк Kuj. 781 Tamapta âkiksortigut $8 \check{5} 5$ " ingmikôrluta 355 " kennunivut . 554 " ômativut . . 688 Tamaptale nippingnik 205 Tamât piniarnimne . 175 taipkoa sivorlit 366 Tammaigiguptigo Jêsuse 728 Tammaisaraidlarapta

Tâmna illa illuptingne 80 " inôtigivavut . 223 " nutaraunerminut 36 " pituarivavut . 366 Tâmnale inûlingipat . 33 Tâmnatauk sinigvingm. 682 Tâpkoa baptijutikut . 602 , innigilikit . . 57 Tâpkonanele attera . 456 Tapserusijatsainarta . 328 Tâpsoma aulapâ . . 42 d. " errilunga . 421 " naglingninga 781 $"$ nâppkiningnin. 708
Tâpsoma pingortitipta 58 Tâpsomanêkuma ter. 378 Tâpsomane peratsainari. 556 Tâpsomangat taimaito 706 Tâpsomunga itterit. . 29 Tâpsomungalo okperкov. 202 Tarne assiojuksauvok . 241 " nagvâromagungne 329 " time Gûdiptingnut 712 Tarnib sattôtiksanganik 241 Tarniga ajoksartoк tor. 388 " Gûdeme pair. 538

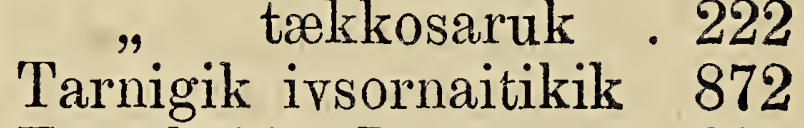
Tarnîk illa Jêsuseme . 338 " imgilaurit : $47 \mathrm{a}$. " Jêsub ikkinginut 300 " Kuviasudlarit . 22 " mannigulerit . 539 " nerriulaurit . . 176 " opallungailaurit 617 " opigosudlarit . 40 " sattortitauvotit. 541 " serrilerit . . 68 " tamaita allakit 503 $"$ tuppalerit . . 49 Tarnikkullo timikkullo 239 Tarnikkut âniagavit • 57 timikkullo . 108 Tarnïma Jêsuse erkarpâ 298 pilloreutiksầ .185 piulijauninga . 854 Tarnimale illinganinga 511 Tarnimigut timimigut . 679 Tarnimnit annitikit. . 229 Tarnimnut tussarnern. 402 Tarninga inûliuk . . 868 
Tarningertoк timiga . 850 Tarninik naglingnik . 463 pairsijotit . . 342

Tarnipkut itsominar . 136 Jêsuseme . 132 kibliktauv . 469 kippipunga . 472 кu viasukp. 464 Tarnipta amnerijangat $833 \mathrm{~b}$. Jêsuse tarnît. 299 naglikpâtit . 563 Tarnise timiselo. . . 211 Tarnit annerimajose .545 Tarnivillo tamaine . . 81 Tarnivulle timivullo .621 Tarnivut angmalerput. 3 ômavut . . . 380 timivullo . . 724 Tatta Engelinit . . 42 d. Tattaminiarpunga erk. 114 Nâl.172d.

Tautuale tækkominar . 90 Tautua tækkominalaung. 54 Tautugungnaerpannik . 400 Tautukkama ajornima. 257 Tautuklugo Kuvianarрок 211 Tautukôrpagit tagvane 111 Tautuvîllo naertillutit 396 Tessiulaunga Nâlegar . 761 Tessiorluarpânga • . 14 Tessiortigut . . . 203 Tessiutsainadlarangne $172 \mathrm{~b}$. Tættigijomadlarpara . 531 Tættigilaptigille . . 710 Tættigitsainaromavagit 537 Tættigitsainarpapiigit . 188 Tættigivagit Jêsuse . . 671
Tætţigivavut Gûde . . 555 Tættiksakadlarkovluta . 154 Tômasetut tækkuta. . 158 Tigusârpaptigut . . . 289 Tikkerkârame nunamut 28 Tikkikunittigut . . 827 Tikkikupko Jêsusiga . 267 Tikkipat Nâlegaк . . 800 Tikkitsomârninga . . 65̃2 Tikkiviksarne . . . 242 Time tarne inôsek . . 782 , tarne pairivâkka 491 Timiga mannigorit . . 838 tarningerpat . 795 Timigale illiktun . . 134 Timigalo tarniga. . 291 Timigatauk piuk . . 770 Timillo pitjutigivavut . 646 Timiminik aungminiglo 612 . aungminik . 608 Timimnik tarnimniglo 768 Timinga nerrigapko . 623 Timingne ikkit arggit 158 Timingnullo ikkilingmut 487 Timipkut sajulerpunga 617 tarnipkullônêt 519 Timise tunnisigik . . 530 Timivit aunga vakkarnit 107 avatinginulle . 646 Timivullo tarnivullo in. 279 Timivut ômativulle . 694 " sinniktillugit 787 Timît inôgutigivavut . 646 Tiptulausijaromârput . 817 Tокко Sâtanelo. . 151 TokKogavit âniavit . 363 
ToKkogavit. Gûdib aung. 252 Tokrojotun-idlunga .132 " ipunga-. 472 Tоккокârtinnanga illa 378 Tokkominut" saimarNalêg. 854 mang. . . . . 257 Tоккоmit makkilaur. . 837 saukat pairij. 154 Tokкоmulle aijotit .110 ToKkomut Jêsub nagl. 381 Kristuse ? 16 Tоккоnît illûnaptingnik 651 Tоккоtaujok timît . . 628 ToKkotigigaptigut ômat 133 ToкKotigingmagit ôma 154 Tоккоviksavut pilerpat 200 Tokrovit âniavillo . . 424. kiblikpâ ômat 135 " manniguidlar. 118 Tokrub kassilininga . 131 Tоккub pitsartuninga . 528 Tokrungnullo ikkingnut 395 Tokkungnut amuartigut 340 Tokkungnut aungnullo 431 Tokkut okpervigivavut 252 Tоккut erkaumadlarlugo 649 Tuksiadlaleravit . . . 88 Tuksialerupta . . 746 Tuksiarnivut Gûde! 693 Jêsuse! . 547 Tuksiarpagit Piulijiga. 408 Tuksiartuksaungilangâ. 185 Tuksiarune Jêsub ittig. 211 Tuksiarvigiguptigo . . 583 Tuksiarvigivaptigittaur 681 Tuksiarviksatôvotit . . 18
Tumise attorpavut . . 861 Tungatikit tokKungnut 876 Tungavezarpoк illa . 433 TunnergutKomuniôneme 652 Tunnininga akkitojoк . 154 TunnitsivigittiguttốK $\quad .716$ TuppaktoK ajornerminik 247 Tussalauruk kennunera 766 Tussakit tuksiarnikka . 216 Tussarkoritse okpertut 820 TussarnernersaujoKar- . niar. . . . . 131 Tussarneromârpok tak. 854 TussarnertokaromârpoK 820 Tussarnertunut nippinut 63 "nippinut 101 Tussarnidlartunik nertor. 654 Tussarpâtit Gûdib . . 525 Tussugidlartakka . . 318

\section{U.}

Ubvarsimajut aungnut. 675 Udsertutsainartiminga . 45 Uerngadlarnermullo . 795 Uigarneк Jesusemik . 708 Uigarnerit tamapta . . 708 Uigarnioniptingne . . 707 Uigarniub Jêsuse . . 708 Uitilaungminga . . 821 Ujarkatut tokkungadlar. 211 Ullapirsartiga aulik. . 356 Piuli. . 224 Ullapirsaumik Kaitsivor 65 tarnip. . 270 sakKềjor 305 
UllapirsautekarpoK Gû. 48 mânna 60

Ullapirsautingnik Jêsuse 294 UllapKosikit nagligi. . 195 Ullapкosertaupat Gûde 74 Unêt idluitogut . . . 81 " okatsiarnanga . 419 " sangêtoksôg. . . 776 Ungagidlarapkille Jês. 394 Ungagigapkit assitit . 521 Ungagivagit, nagli. . 288 Ungajub tâpsominga . 272 Ungamut næglikaminga 22 Ungasiktullo illingnit . 669 Unnuame kingorlerpâme 442 Unnuak ussornartok . 59 Unnulermat rejungmit 117 Unnukpat sinnijomag . 758 Unnungme merngoêrser 794 Unnungmut kiksadlaru. 223 Uvagulle kinauvita . . 546 Uvagut tamapta . . . 567 Uvaguttauk . . $42 \mathrm{a}$. Uvamnigle pairksigavit 306 Uvamnuttôk Kaisâdlaler 213 Uvanga kinauvîk? . . 628 Uvangatauk ômatimne 66 Uvaptitulle ilaungmat 678 Uvinekakattivut! . . 670 Uviniga illa . . . 572 Uvinik auglo tâpkoatuak 54 Uviningme inôvogut . 698 $"$ sakkernit . 53
Uviningmêlauravit • . 248 Uvingub issumangit . 838 Uviniptingnik attijoK 256 Uvlâkut kaulerangat 170 tuppalerangapta 681 tuppaleruma 758 Uvlok ketterarêrmat . 92 " tachesimavor . 795 " taimna issumagig. 115 Uvlome apkotiksavut 37 , erniangojor . . 32 " illalioruk . . 28 " Jêsuse . . . 770 " nutâmut Jâr . 78 " pairilaungminga 758 " piksaptingnik . 190 " pivor nunamut 45 " sivorlerpâmik . 70 , sulliæksakka 766 Uvlomelo sulle tussar. 476 Uvlullo unnuallo . . $158 \mathrm{~b}$. Uvlut nelliutikpatta . 140 " tamaita âniat . 71 ” tamaita ikkingne 636 " tamaita imailaung. . . . 640 tamaita tokkut 791 Uvlưut nâkârtinnagit 516

\section{置。}

Zionemut Nâlegavut 46 


\section{Zugabe}

\section{Sankey'sche Lieder enthaltend.}

Die meisten Melodien nach einer deutschen Berner Ausgabe.

\section{Tuksiarutsit illangit,}

Moodyb Sankeyblo attorkattalauktangit.

1. Orpertut sorsulstut sâlakarningat. Tæk. 2,25 .

Ho! my comrades, see the signal.

Auf, ihr Brüder, seht das Zeichen.

1. Illakka, maksualaukta

sorsungniptingne!

Ikajortivut kanningmat

ajugauvlune.

"Sôngotitse, Kaidlarama!“6

Jêsus niplerpok.

" „Nâlegak, angerpaptigit

",Sôngotittigut!““"

2. Akkeravut sorsudlartut

erksinadlarput.

Sâlakarniarkôrmatta

mungulerpogut.

"Sôngotitse kaidlarama"

malliktingillo.

3. Tiptulausijartokarpoк

Kannitaptingne; 


\section{Tuksiarutsit illangit.}

Jêsumut âkiksimangmat

sâlakarnivut.

Sôngotitse, Kaidlarama"

malliktingillo.

2. Upkuêrutjaunivut. Tæk. 21, 25.

There is a gate that stands ajar.

Geöffnet stehet eine Thür.

1. UpkuêrsimavoK kilak,

Jêsub sakкijârvia,

Jêsub ikkiliksôdlartub,

Pivluta tokкolauktub.

Nâpkigijauneк opinak!

Upkuêrutjaudlarama

Uvangatauk,

Illa uvangatauk.

2. Upkuêrutjauvut illa

Piulijaujomajut,

Aklujut akluitullo,

Nunalît illûnaita.

Nâpkigijauner opinak!

malliktingillo.

3. Upkuêrmat, ittidlarit,

Kimut agviartaunak!

KappiasûtiksatittauK

Kunnujutiginagit!

Nâpkigijaunek opinar!

malliktingillo.

Kappiasûtiksat pâne

Jêsumut pêrtaulârput;

Naglingnermut ônarluta 
Kujagidlalârpavut.

Nâpkigijauneк оріnaк!

malliktingillo.

3. Jêsuse Kàngerpok. Luk. 18, 36-38.

What means this eager, anxious throng.

Was ist das Volk so eifrig dort.

1. Inuksuit sôg kaut tamât

Tagvunga akpangidlarkât?

Sumîk tækkojomavlutîk?

Sôg nimniutidlarlutîk?

Tagva kiojokalerpoк :

"Jềsuse ovane kângerpor!"

2. Angut imna aulatsijor

Inungnik, tattamnadlartok,

Orâlatsiartor imna

Iglugasængne kinauvâ?

Ama niplertokadlarpoк:

„Jêsuse ovane rângerpor!"

3. Jêsub illa taipsomane

Nuname inôlaurame

Ikajorpait Kannimajut,

Tumângajut, tautungitut.

Kuvê! niplertokalauкрок :

"Jêsuse ovane kângerpor!"

4. Kaivorlo sulle uvlome

Inungnik pitsiarlune.

Illûnâne tækkonâkit

Piulijipta tumingit!

Sulle niplertokadlarpor:

„Jêsuse ovane rângerpor !“ 
5. Tagva kappiasuktose

Kaititsetôk illûnase!

Pijaugupse ajornernut, Jêsumut pêjartaulerput.

Kuvê! niplertoradlarpor :

"Jêsuse ovane kângerpor !“

6. Piulijîlle Jêsuse

Inuk! ajaktudlarungne, Aunganik nacholeruvit, tagva kingurailârpotit! Kappê! niplertoKadlarpok : "Jêsuse kângersimavok !“

4. Terlingnartomêdlune. 5 Môs. 33, 27.

Save in the arms of Jesus.

Sicher in Jesu Armen.

1. Terlingnartomêdlune

Jêsub sagvingane,

Tâpsoma tallîgingne

Kuvianadlarmềk!

Engelit imgerningit

Tussarpakka illa

Kuvianartovingmit

- Pânêtomit tagva.

Terlingnartomêdlune

Jêsub sagvingane,

Tâpsoma tallîgingne

Kuvianadlarmểk!

2. Siarklernêjarlune

Jêsub sagvingane,

Sumut pijaulugane

Kuvianadlarmêk !

Okumaitut tamaiti 
Okiglijaudlarput!

Kassilinaertitauvut

Kappiagijavut.

Terlingnartomêdlune malliktingillo.

3. Jêsus erdligijara

Akkisartortiga,

Nangervigênarlagit,

Kairtoridlarapkit.

Illingnit nûtsænnanga

Unnuangodlarmat

Kaulersềjomavunga

Keblitsiartomik.

Terlingnartomêdlune

malliktingillo.

\section{Piulijannerub utakrijauninga.}

Mein'n Geist, mein'n Leib und Seele.

1. Time anerneralo

Tunnijomavâkka,

Illingnut Jêsusiga

Perkutaurkovlugilk.

Jêsus utakrivunga

Piuliklernernik,

Utakkidlarpunga

Piuliklernernik.

2. Aungnut ubvartauvlunga

Perrutigivarma,

Ivsornaititaulanga

Anernernut illa.

Jêsus utakrivunga

Piuliklernernik,

Uttakkidlarpunga

Piuliklernernik. 


\section{T'uksiarutsit illangit.}

\section{Nâlagit! Tæk. 3, 20.}

Knocking :,: who is there.

Horch, es klopft! komm herein.

1. Nâlægit! nâlægit!

Kailertor kinaunâ

Avilortartok ômamut?

Jêsuseovor illa

Itteromajok illingnut.

Upkuêrutilauruk!

2. Nâlægit, Kailerpoк.

Tækkuit, mânêpor.

Kemmergolikit aggangit

Puttumajut pivlutit!

Illaliulauruk illa

Illingnit nûkonnago.

\%. Kittorngat piulijaudlartose. Rôm. 3, 24.

Free from the law, o happy condition.

Frei vom Gesetz, o seliges Leben.

1. Kittorngat piulijaudlartose

Jêsub aungat pitsartuninganut,

Ajortunit kellujaujose

Jếsusipta tokrunganut.

Kôr.: Nâlægit, tussaritse okpertuarupse piulijauvose

Jêsub kikikiktortauninganut.

Attê kammagidlarsiuk.

2. Jêsub Kaikovâse okarlune :

„Kaititse uvamnut ajortose,

„Nekoksitidlaromavapse

"Piloriktiluse ila."

Kôr.: Nâlægit malliktingillo. 
3. Tappanase aivigidlarsiuk, Kittorngartut illaliudlarpâse Nâlegauvingmut pânêtomut Issokangitorsoarmut. Kôr.: Nâlægit malliktingillo.

8. Utterittôx! Luk. 15, 18.

Come home. - Komm heim.

1. Utterittôx! tammarsimajotit Tâksiortotillo kuviasûteKarnak. Assiojotit kait, kait, utterit Kait, kait Jêsusemut!

2. Utterittôk ! utakкijauvotit Karkialerlutit uttisâlaurittôk ! Assiojotit malliktingillo.

3. Utterittôk! Atâtait kilangme. Nagligosudlarmat nacholungitorlo. Assiojotit malliktingillo.

\section{Ikkârtitausênivut.}

Wartend stehen wir am Flusse.

1. Nunamêniptingne illa Kôb sinnânêkorpogut, Ikkârtitausêvlutalo Piloringnartovingmut.

2. Akkianut tækkoluta Ajorsaralloarpogut; Engelille tussarpavut Nertordlertut Gûmik. 


\section{Tuksiarutsik illangit.}

3. Tussaumakattarlutalo

Iglugasæksuit nutât

Pinnarnadlarningænnik

Kaumajoksôdlarlutik.

4. Illavut angergarsimajut

'Taikane tækkolugit

Nerriudlarpogut illa

Illagêktitauvluta.

5. Tagva sokrosijuiluta

Attavigênarlavut

Nagliktor piulijivut

Kikkitaulerkonnata.

\section{Sikjatsiar кeblertak.}

My days are gliding swiftly by.

1. Uvlukka tingerkôdlarput!

Siorniornarmattale

Tamâne tujormivimne,

Nokкarkolungilakka.

Kôngme mâne nangerpogut,

Illavut ikkârêrput;

Akkianit sikjatsiar

Keblertak saKкijârpoк.

2. Parngnaita, makkitiptigut

Tapserusijarluta,

Kollivut âkiksorlugit,

Uiksak каikojingmat.

Kôngme mâne nangerpogut

malliktingillo.

3. Sulle kappiasukkupta, Mungunganiarata! 


\section{Tuksiarutsik illangit.}

Pâne ullapitaunata

Merngoêrservekarapta.

Kôngme mâne nangerpogut malliktingillo.

4. Nertornarta! akkianut Gûmut кaikojauvogut. Tagga illa igluksavut IssoKatsængitomut. Kôngme mâne nangerpogut, Illavut ikkârêrput; Akkianit sikjatsiar Keblertak sakкijârpoк.

II. Aputituttôn! Img. 51,9.

Whiter than snow.

Weiss wie Schnee.

1. Illûnapkut ivsornaitsomavunga Jêsus pigilutit sokrosernanga; Kelaumgujamnigle pêjaiviginga, Ubvanga, aputitut KaKkortinga! Aputitut! apumit illa Kakкornersautilunga ubvalaunga!

2. Nâmangitut, Jêsuse, assserukit, Mikkinerpâttauk annajaginagit! Illûnapkut pigisinnalilaunga! Ubvanga, aputitut kakkortinga ! malliktingillo.

3. Nâlegan Jêsuse, utaknivagit, Ómatetârtinga illumne igvit! Tussaruk kennujub tuksiarninga! Ubvanga malliktingillo. 


\section{Tuksiarutsik illangit.}

4. Pâmangavigivlutit kennuvunga

Pijaksautsængigallortôjunga,

Aungnigle, aungnigle tættekarpunga.

Ubvanga malliktingillo.

5. Okpernikut kennujutigijara

Pivara; ômatetârtidlarparma

Onarsititaujomik naglingnermut,

Ubvarma itilunga aputitut.

Aputitut; apumit illa

KakKornersautilunga ubvarparma. 



\section{I}

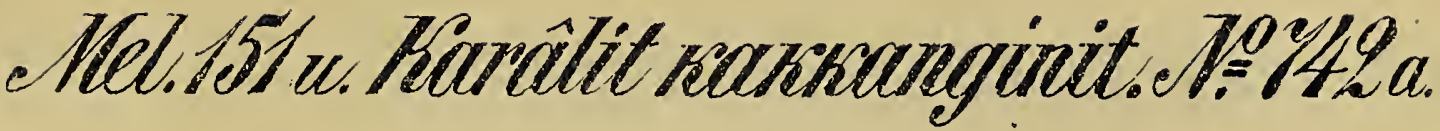

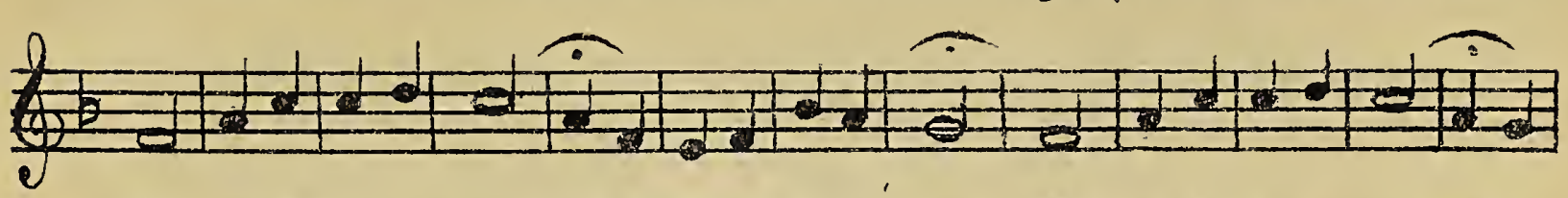

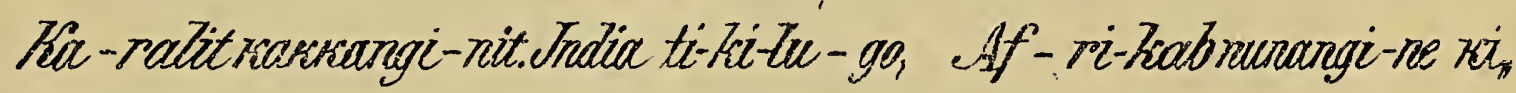

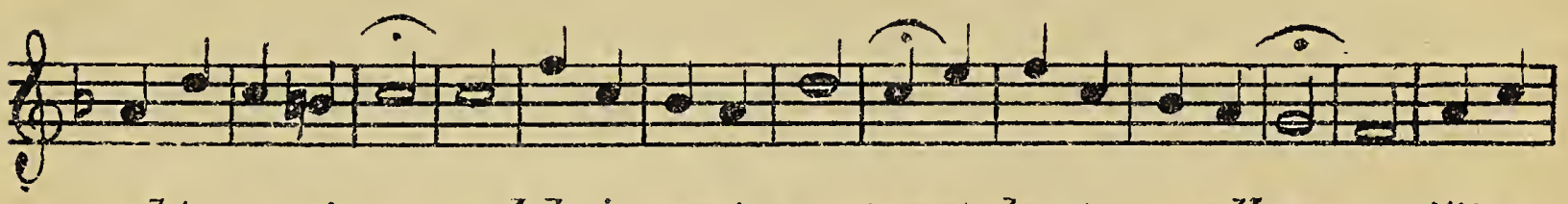

arta-reurtume, net-7o-ju-ne ta-maine nizler-to- scadlarnos:raitit-st.

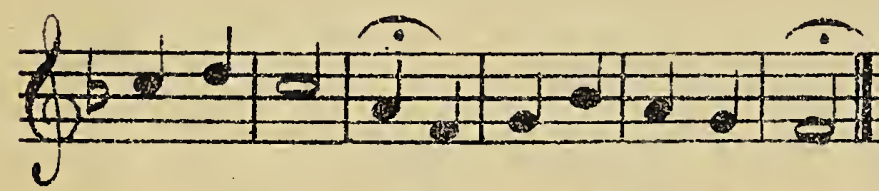

pêrsi - tit - se keluumgujaptingnits.

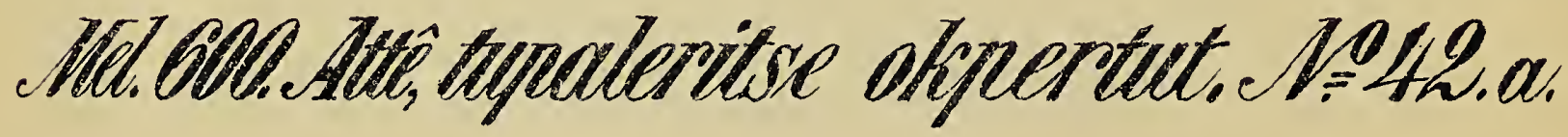

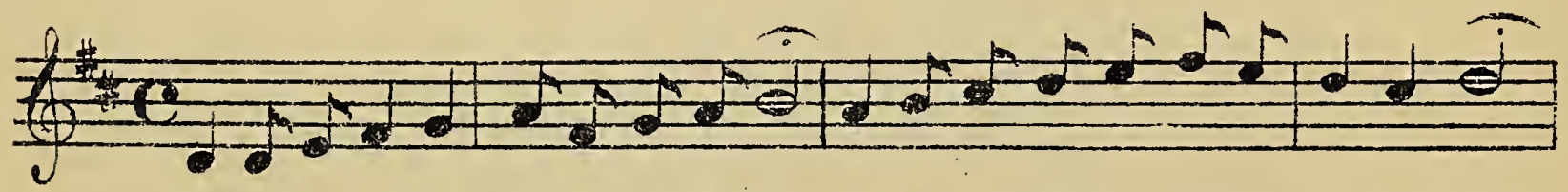

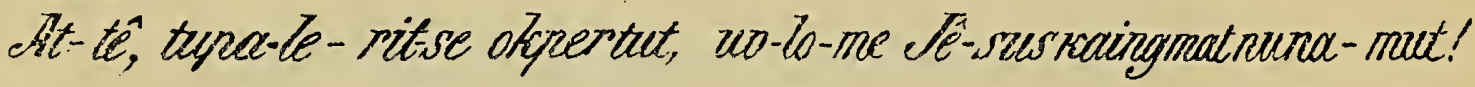

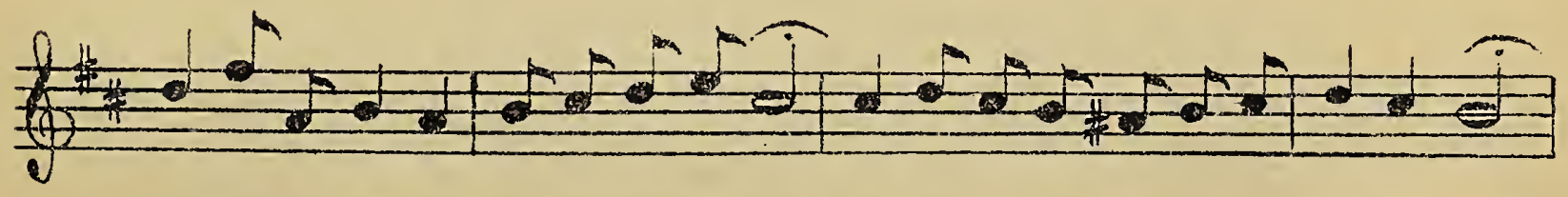

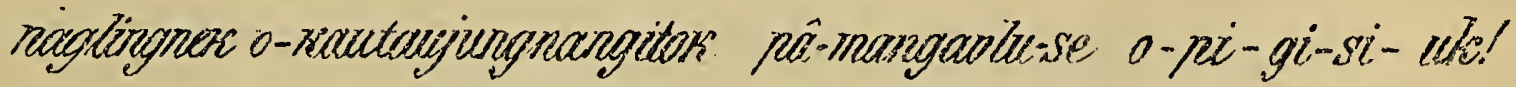




\section{II}

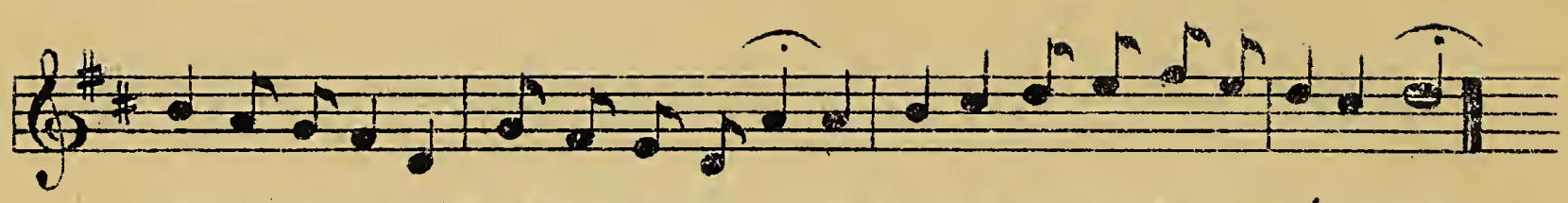

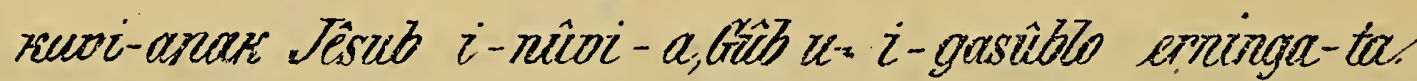

\section{Oligertut sorsuktut sêllarsarningat.}

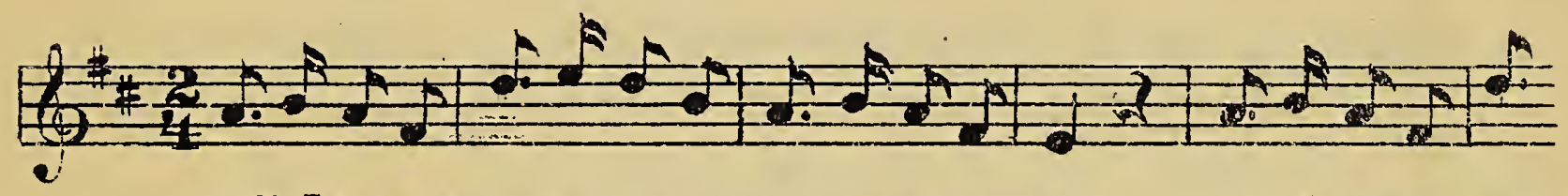
Mllakiza mak-su-a-Louktasorsurgniptingne, $\quad$ i-kajonti- out

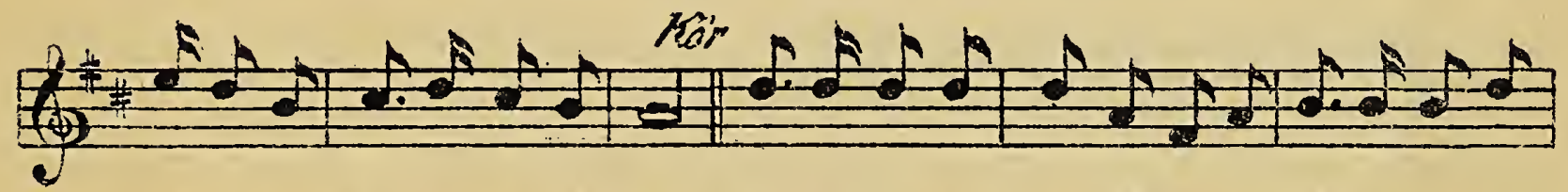

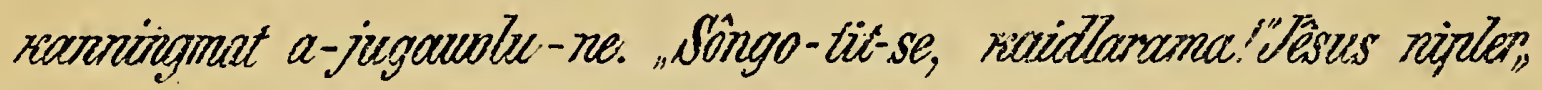

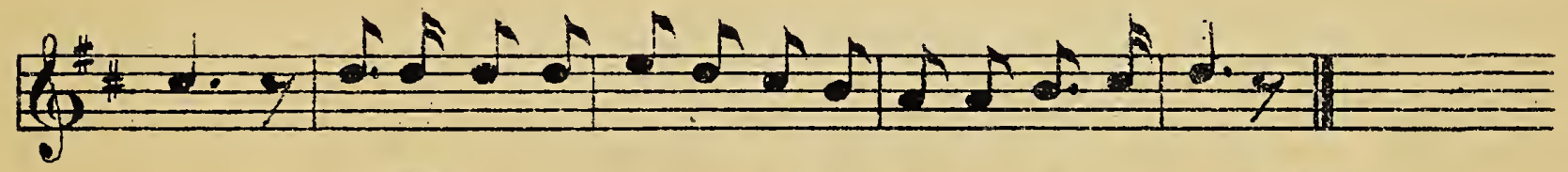

"ror. "„Nâtegar, angernapti-git,sôngo-tit- ti-gut!"

\section{Vpruêrufjaunimut.}

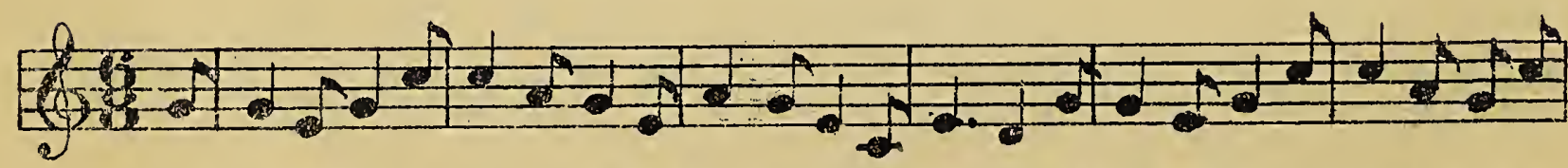

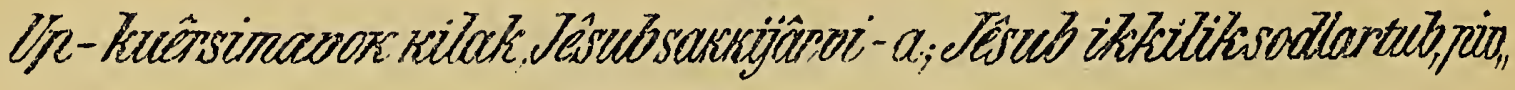




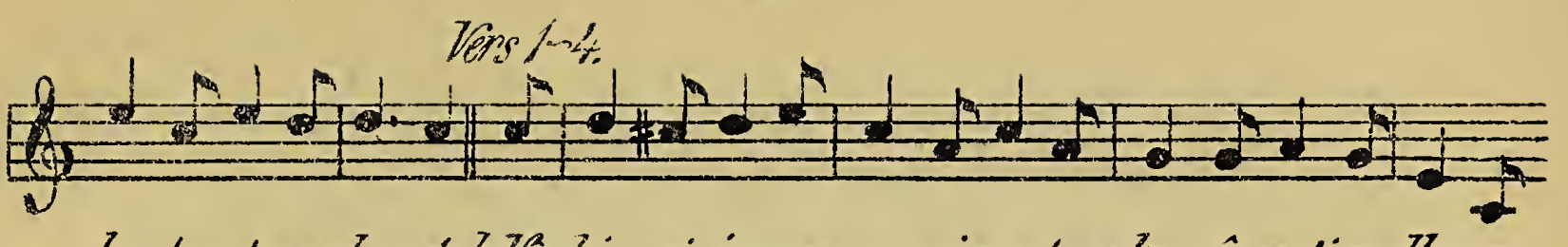

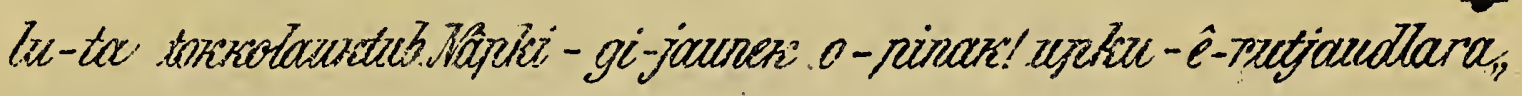

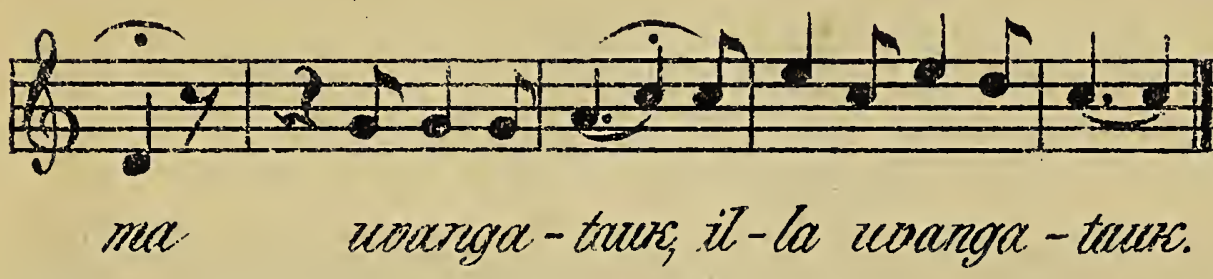

\section{e. Têsuse raingerpor:}

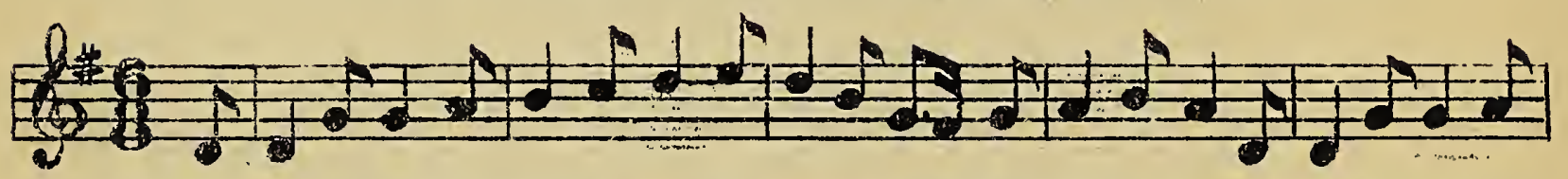

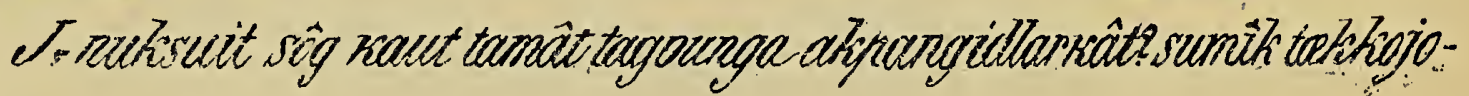

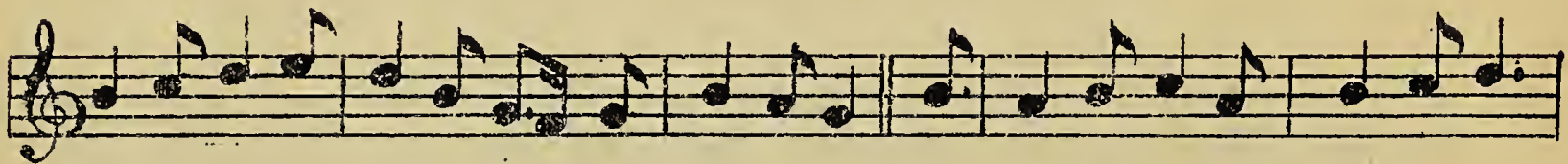

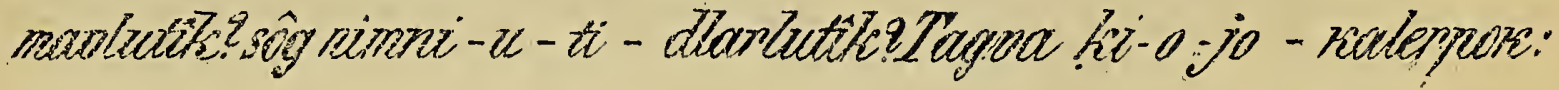

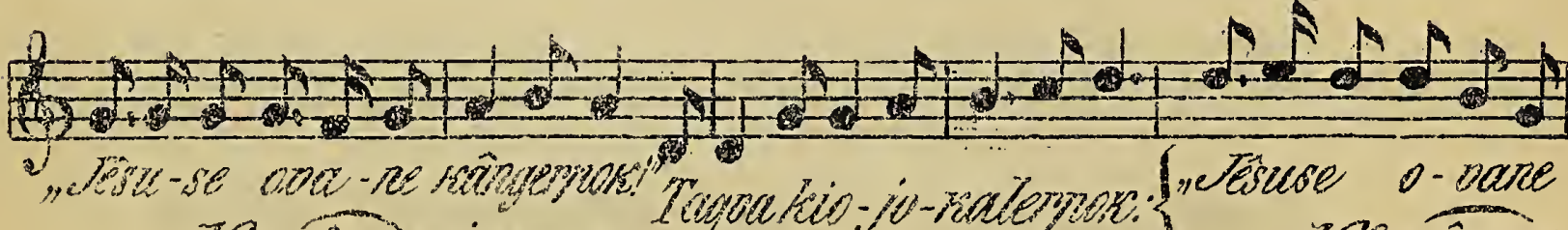

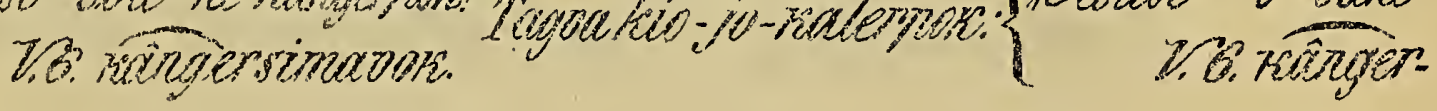

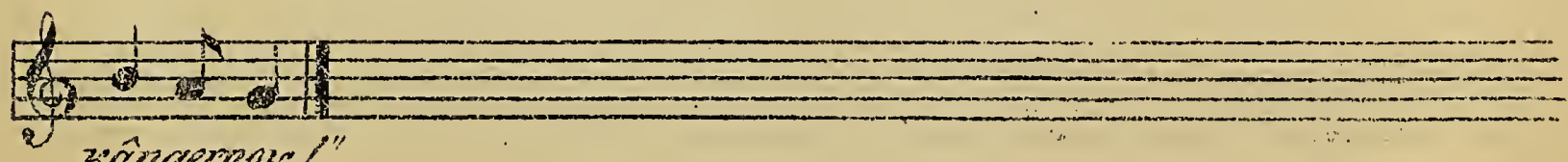
"rângerpose!"

simanok: 


\section{Terlingnartorsédluse.}

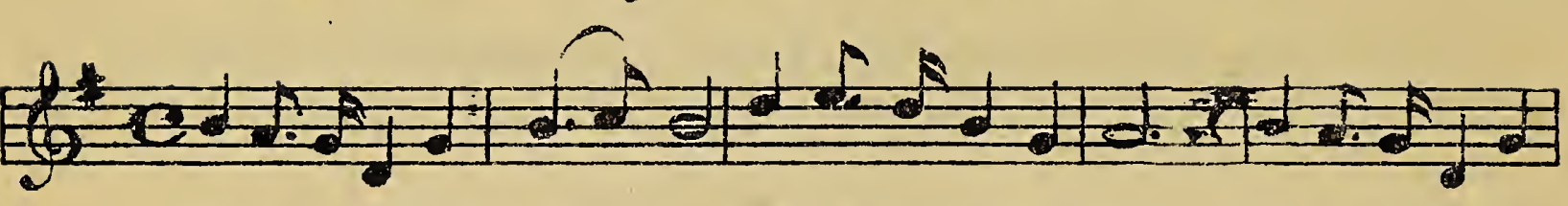

Ierlingnartomê-allune Jesub sagningone, tâpsoma talli

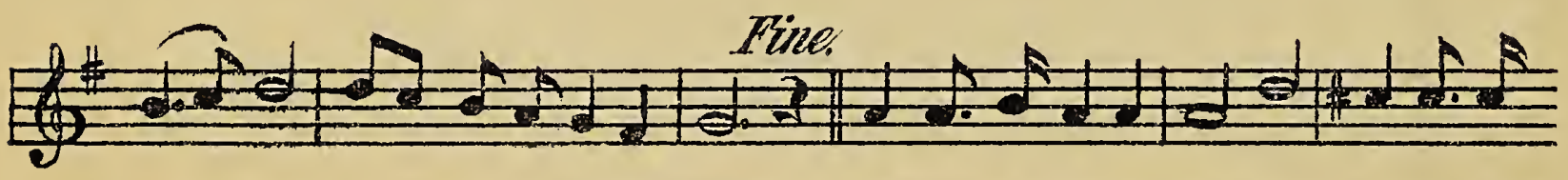

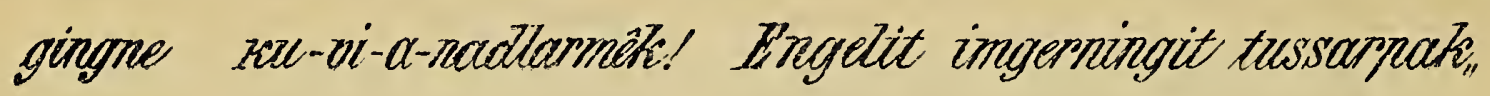

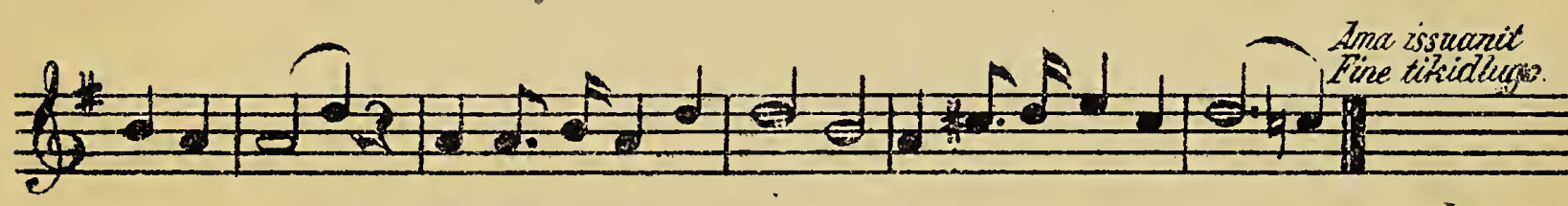

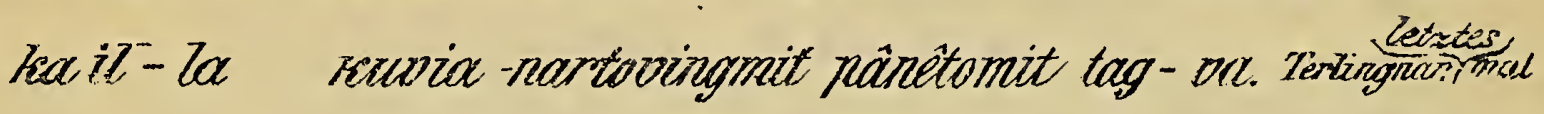

5. Piuljounetub utancijauninga?

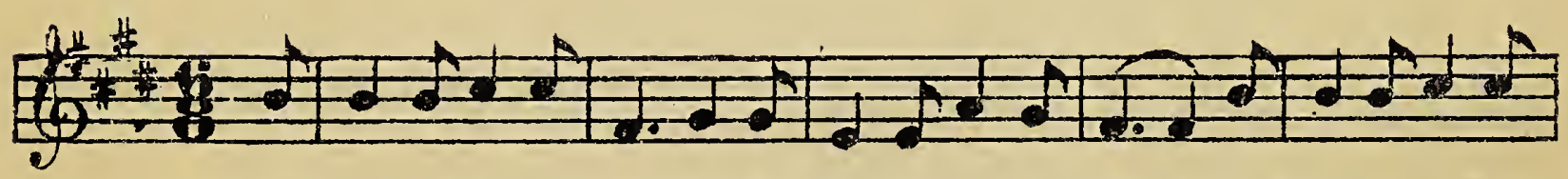

Ti me anerne-ra-to tunni-joma-oâk-Ka, itlingmutetesu,

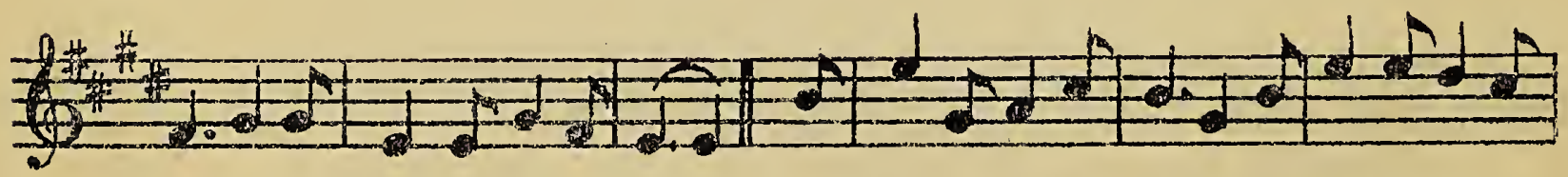

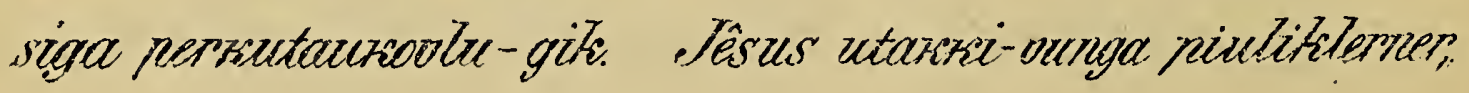




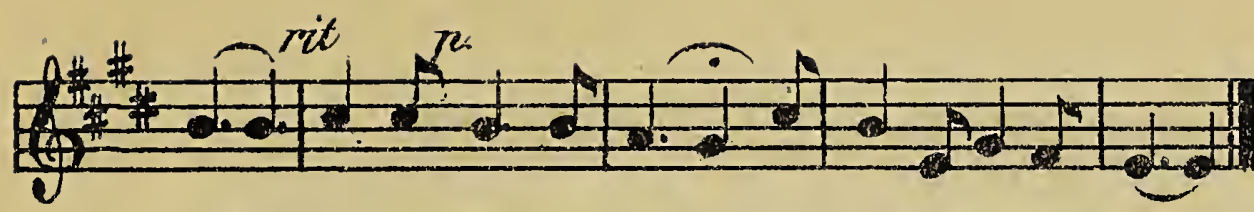

nits, utacksidlarpunga pi-u-li-jauner mits.

\section{G. Nâlagit.}

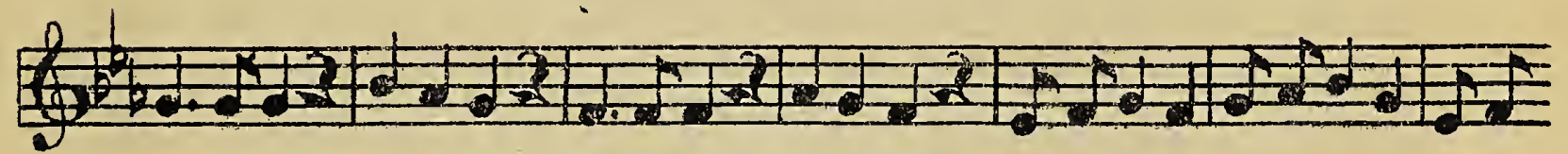

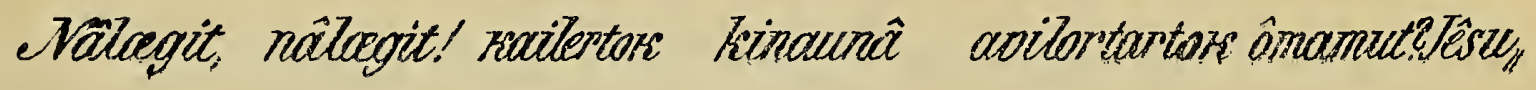

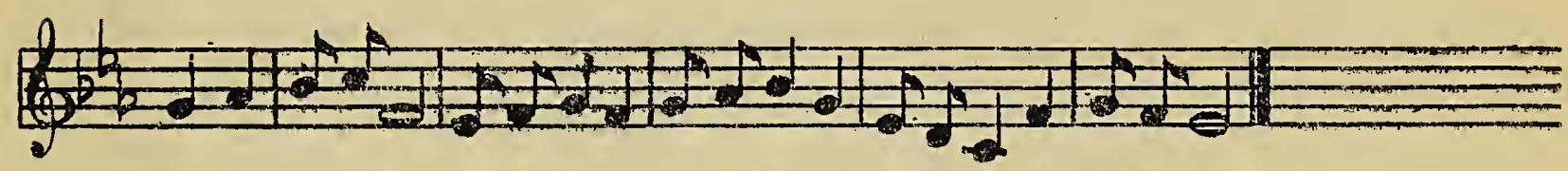

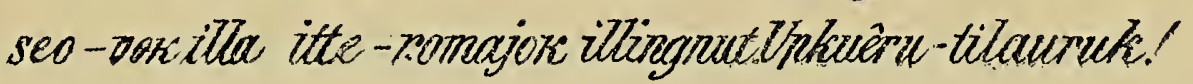

\section{\%. Auttorngat piulyoudlartose.}

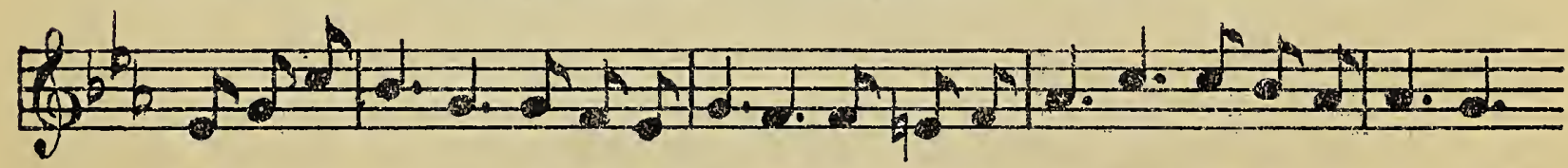

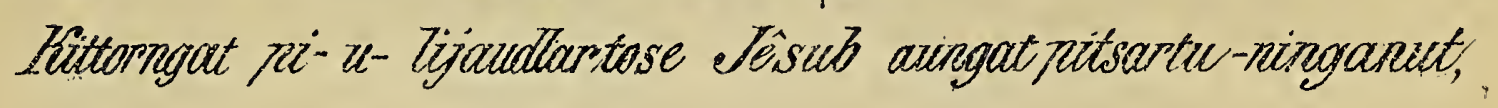

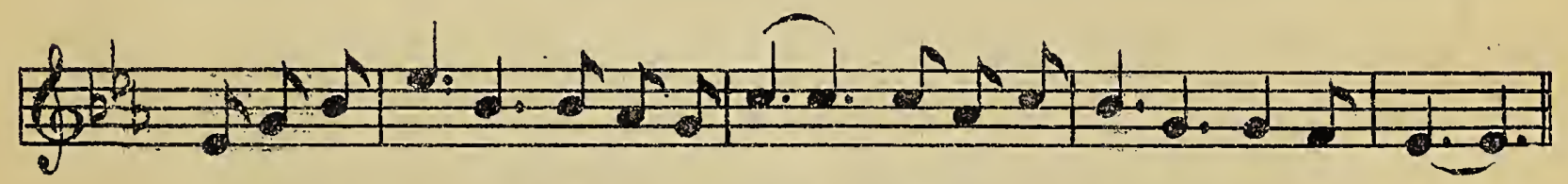

giertu - nit kel-bigayo - se Jesw-sig-tatorkunga - nut.

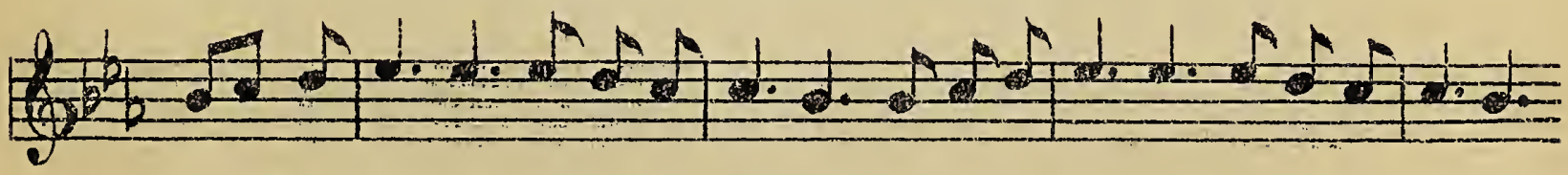

-Na-las-git, lus-saritse oferer-tu-arup-se ni-u-lijau-base 


\section{VT}

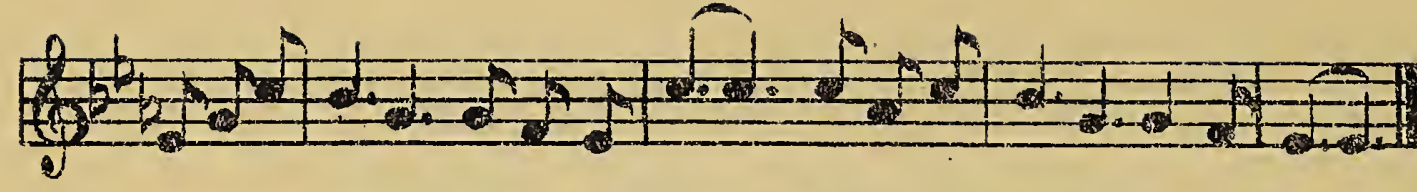

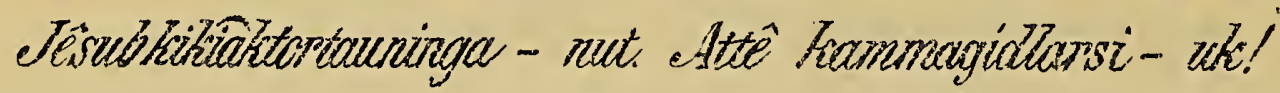

\section{Vtteritton:}

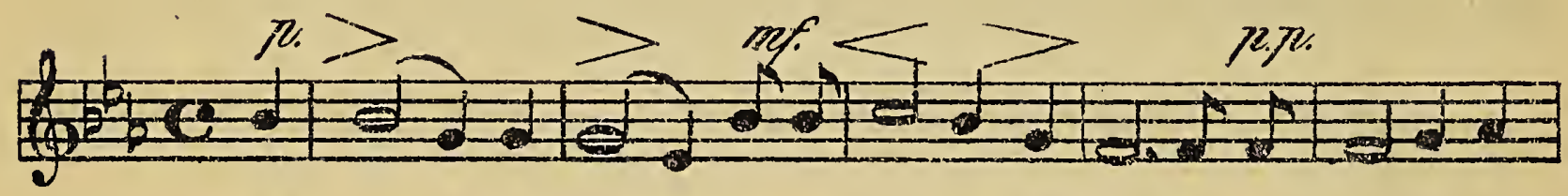

$V t$ - te - rit- tori! tammar-si-majo-tit tarksi - or-ta-tiln

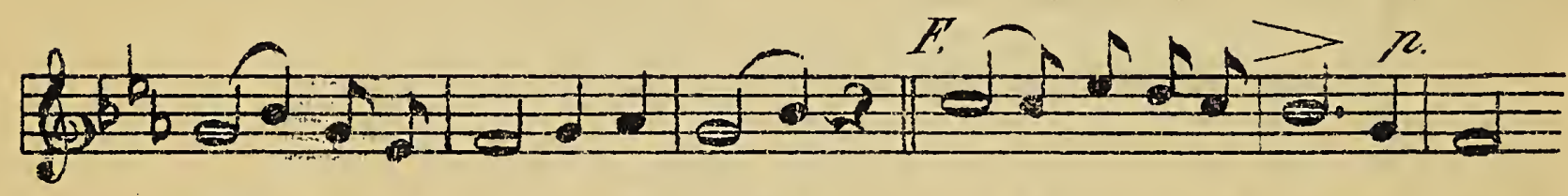

lo kwôa-sû-te-siar-raks. $A s-s i-0$-jo-tit kait, nait,

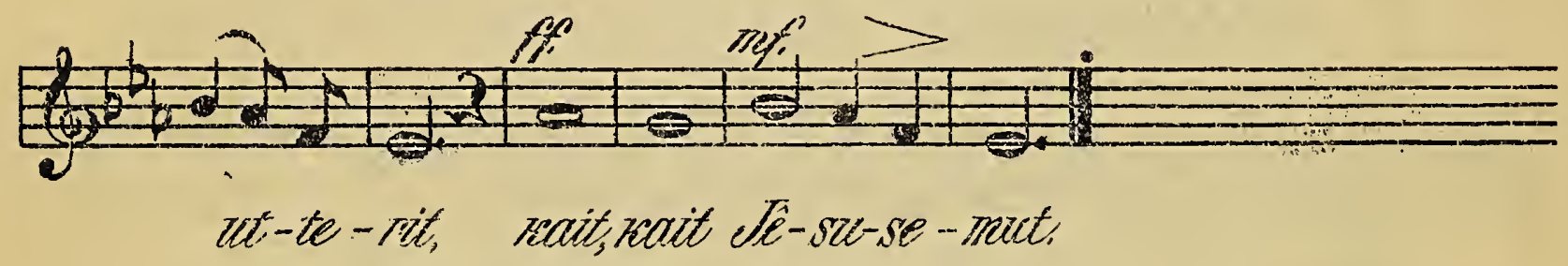

\section{e. ethkîrtítausénimut.}

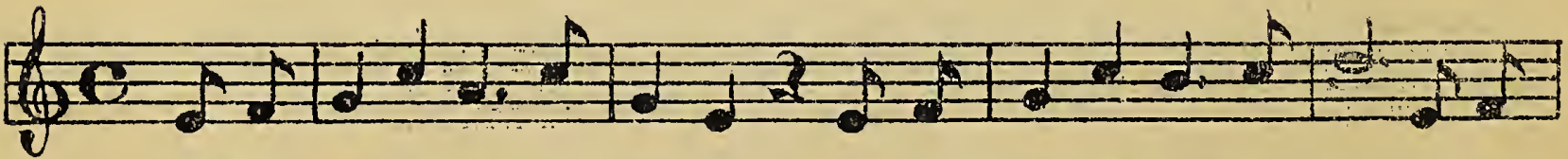

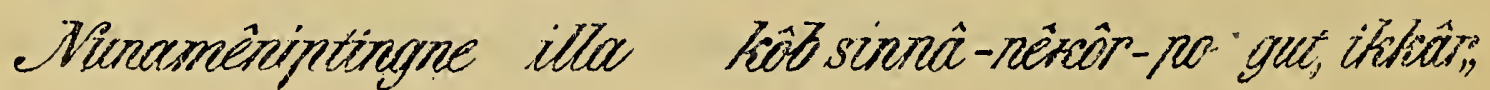


VII

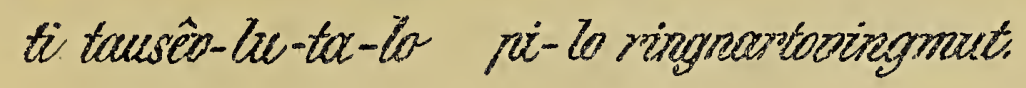

10. Sityatsian keblentar.

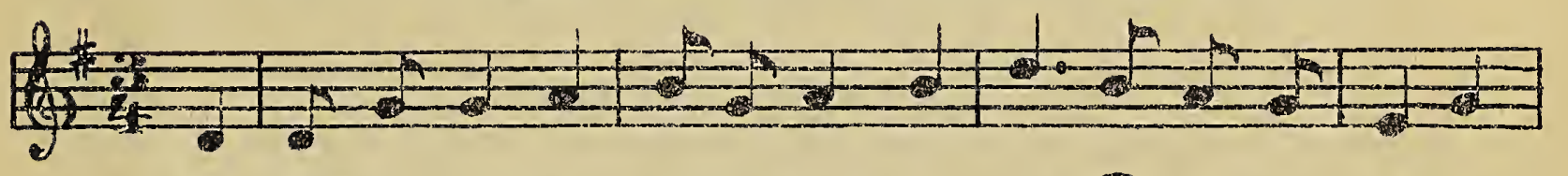

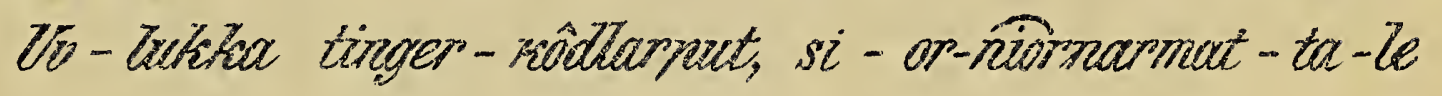

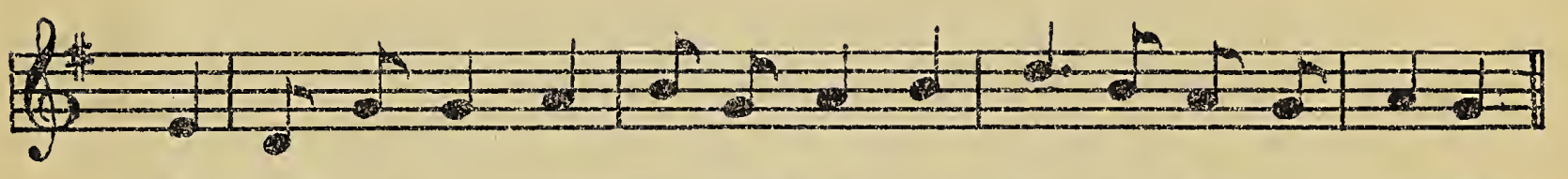

ta-mâ-ne tu-jor-mi-vim-ne nos-kar-ko-lu-ngi-Zariza.

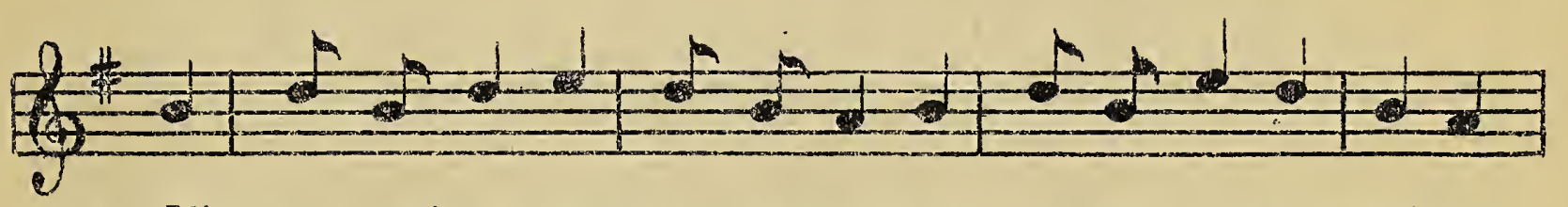

0.1-4. hiôngme mâxe nanger-po-gut, it - Laout itchex-rêrput;

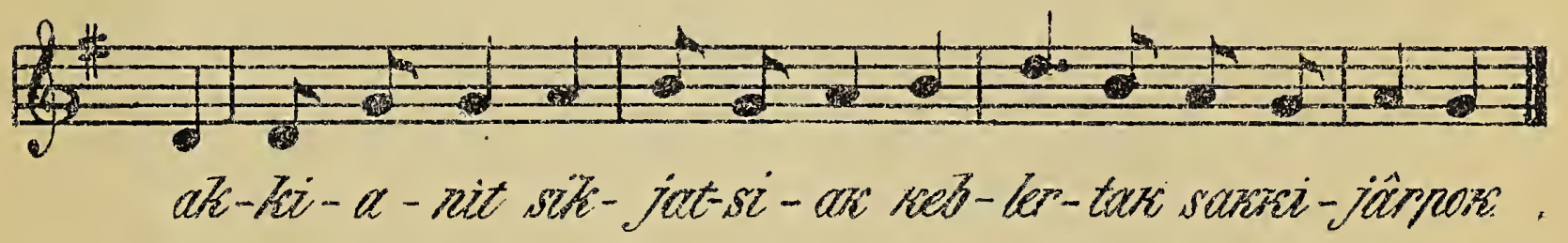

1. Aputituttồ?

Mi- Tûnapkutiosornaitsomaonunga Tèsus pi-gi-lutit sorn, 


\section{VIII}

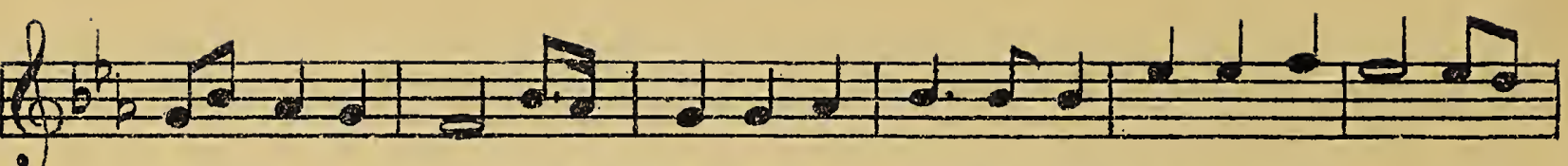

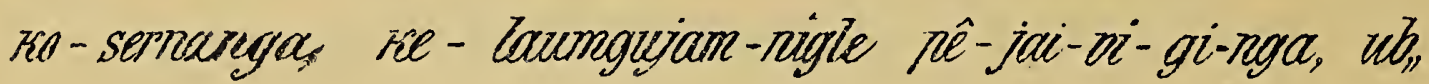

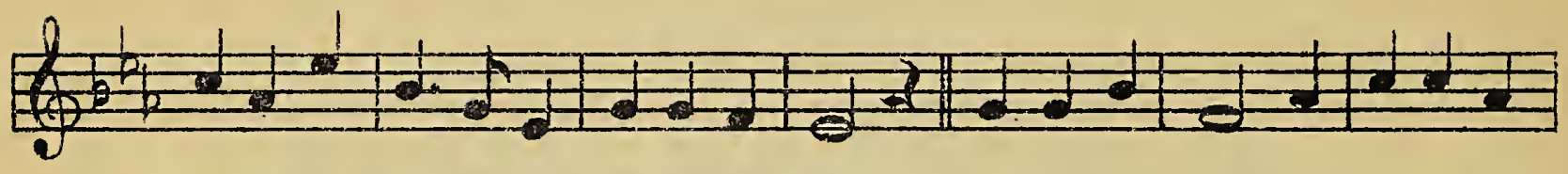
vanga, a-pu-ti-tut zakrsortinga!' a-pu-ti-tut! a-pumit it,

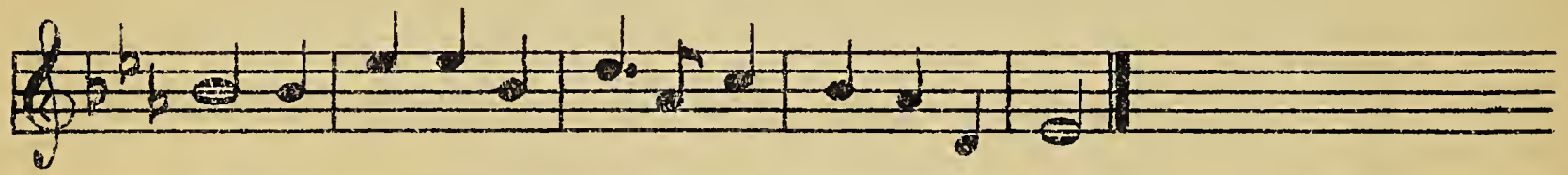

la saxkorkersau-ti-burga wbotounga! 

Microformed by

Preservation

\section{Services}

min $\frac{651205}{\text { June } 111994}$ 


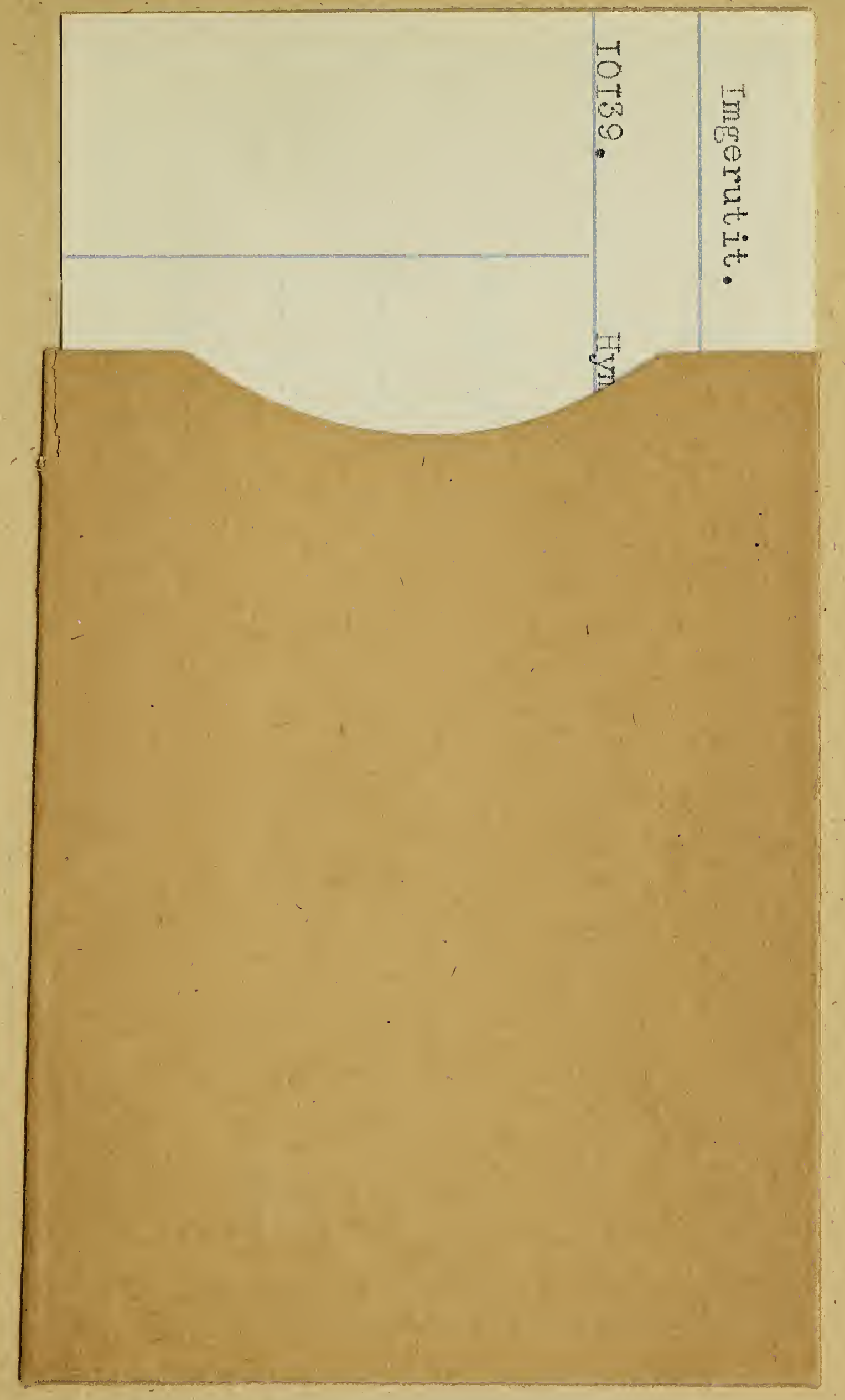




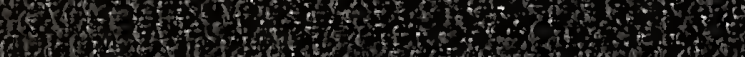

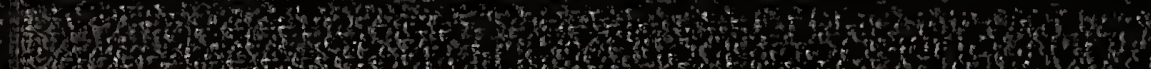

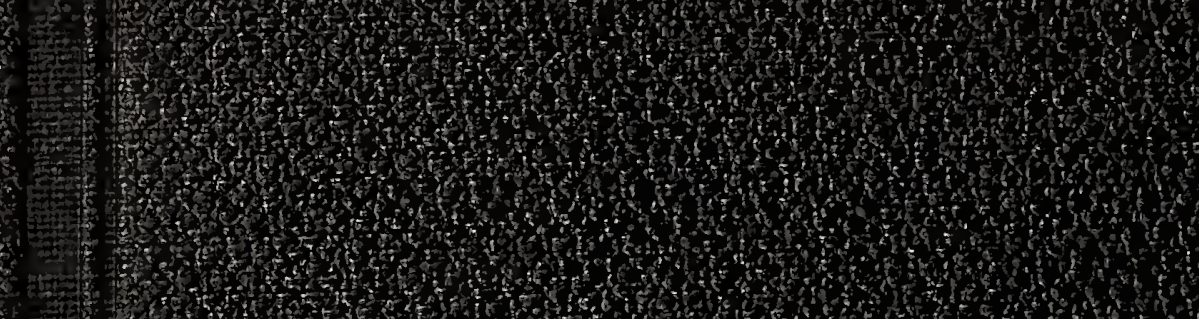

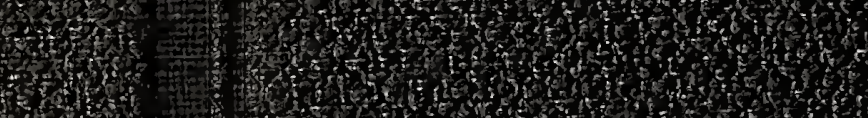

(3)

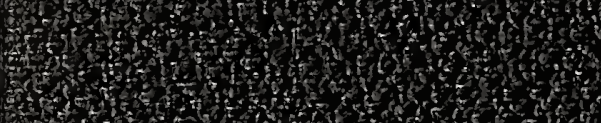

H.t.

(2)

Tht

$7 x^{2}$

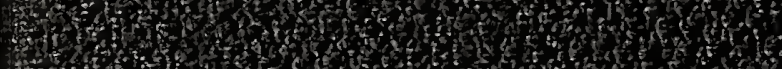

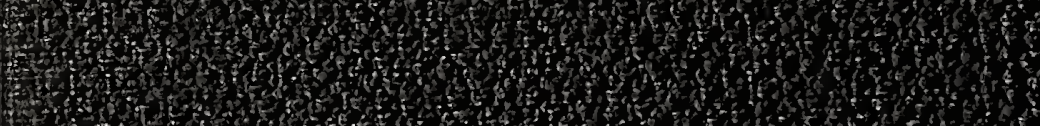

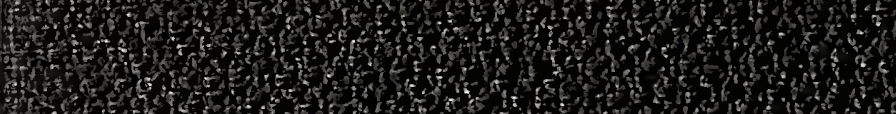

W. W 5 W

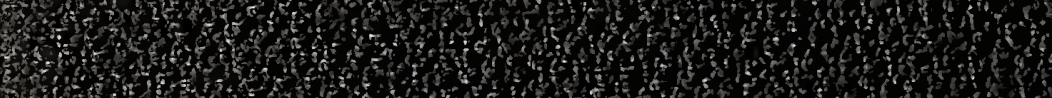

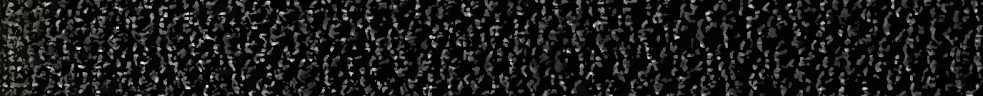

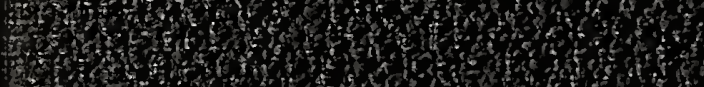

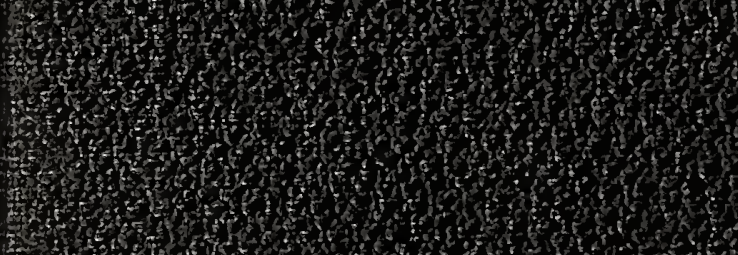

2.

H. W

W. W

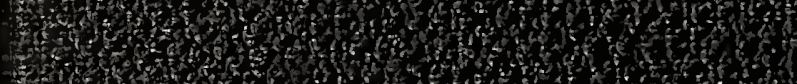

7.

13.

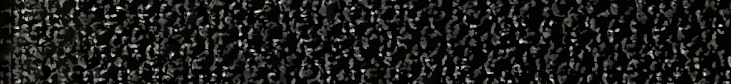

H.

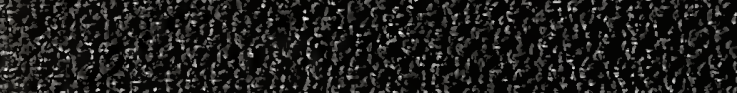

W

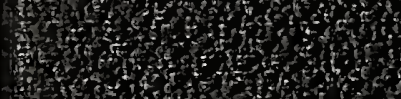

3. Whom

3.

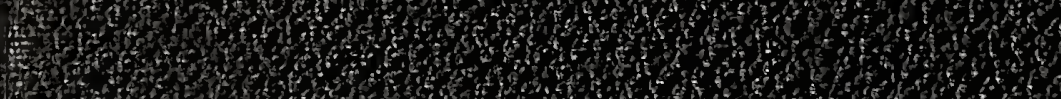

13.

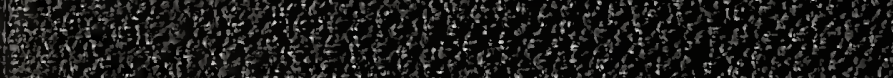

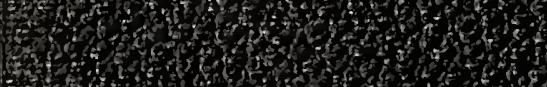

H.

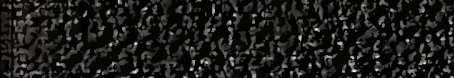

1.

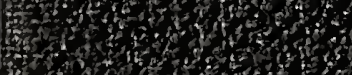

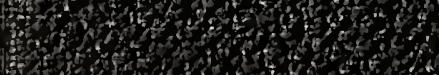

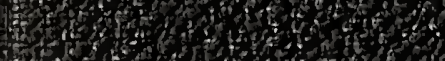

